\title{
Estabilidade Estrutural e Bifurcações de Campos de Vetores Descontínuos
}

\author{
Ana Lúcia Fernandes Machado
}

TESE APRESENTADA

$\mathrm{AO}$

INSTITUTO DE MATEMÁTICA E ESTATÍSTICA

DA

UNIVERSIDADE DE SÃO PAULO

PARA OBTENÇÃO DO GRAU DE

DOUTOR EM

MATEMÁTICA APLICADA

Orientador: Prof. Dr. Jorge Manuel Sotomayor Tello

São Paulo, Dezembro de 2000.

Área de Concentração: Equações Diferenciais e Sistemas Dinâmicos

A autora desta tese foi bolsista da FAPESP - Processo: 96/1435-3 - durante

o período de abril de 1996 a março de 1997. 


\section{Estabilidade Estrutural e Bifurcações de Campos de Vetores Descontínuos}

Este exemplar corresponde à redação final da tese devidamente corrigida e defendida por Ana Lúcia Fernandes Machado

e aprovada pela comissão julgadora.

São Paulo, 8 de dezembro de 2000.

Banca examinadora:

- Prof. Dr. Jorge Manuel Sotomayor Tello (orientador) (IME-USP)

- Prof. Dr. Clodoaldo Grotta Ragazzo (IME-USP)

- Prof. Dr. Marco Antonio Teixeira (IMECC-UNICAMP)

- Prof. Dr. Carlos Teobaldo Gutiérrez Vidalón (ICMC-USP)

- Prof. Dr. Ronaldo Garcia (UFG) 
Para o Fernando. 


\section{Agradecimentos}

Ao Prof. Dr. Jorge M. Sotomayor Tello pela sua estimulante orientação, e pela sua dedicação e paciência ímpares, mostrando-se sempre disponível e atencioso para a discussão da temática proposta, tornando possível a consecução deste trabalho.

Ao Prof. Dr. Marco Antonio Teixeira (IMEC-UNICAMP) que, na fase inicial do presente trabalho, recebeu-me atenciosamente, por tantas vezes, para discutirmos aspectos do trabalho.

A minha família e aos meus amigos pelo apoio, em especial aos amigos Messias, Roberto e Luiz Fernando.

Ao meu marido Fernando, pelo apoio e paciência que teve comigo durante a realização deste trabalho. 
Seja M um domínio, dividido em duas partes, $\mathrm{N}$ e $\mathrm{S}$, utilizando-se uma função diferenciável $f$. Seja $\mathrm{D}$ a fronteira comum de $\mathrm{N}$ e $\mathrm{S}$. Em $\mathrm{N}$ é definido um campo vetorial $\mathrm{X}$ e em $\mathrm{S}$ um campo vetorial $\mathrm{Y}$, formando assim um campo $Z$ definido em $M$, chamado de campo vetorial descontínuo. Filippov [Fi] desenvolveu as regras para a transição das órbitas entre as regiões $\mathrm{N}$ e $\mathrm{S}$ ou a permanência das mesmas em $\mathrm{D}$. Sotomayor e Teixeira [S-T] utilizaram como domínio a esfera $\mathrm{S}^{2}$ e desenvolveram o método da regularização. Este método consiste em utilizar uma função de transição $\varphi$ para formar uma família de campos vetoriais contínuos que se aproxima do campo vetorial descontínuo, quando o parâmetro $\varepsilon$ tende a zero. Estes campos vetoriais contínuos são chamados de campos vetoriais regularizados. Sotomayor e Teixeira estabeleceram condições sobre $Z$ para que os campos vetoriais regularizados sejam estruturalmente estáveis. Neste trabalho utiliza-se como domínio uma região $\mathrm{M}$ compacta e contida em $\mathfrak{R}^{2}$. E, utilizando-se o método da regularização, estabelece-se condições sobre X e Y para que os campos regularizados sejam estruturalmente estáveis. Neste caso foi acrescentado o problema do tratamento dos pontos $p \in D \cap \partial \mathrm{M}$. Prova-se que o conjunto de campos vetoriais descontínuos que satisfaz estas condições é genérico dentro do conjunto de campos vetoriais descontínuos, utilizando-se translações e rotações. Inicia-se o estudo das bifurcações, contrariando-se uma das condições estabelecidas, a de que os campos X e Y não possuem singularidades em $\mathrm{D}$, e verificando-se como é a família de campos regularizados neste caso. 


\begin{abstract}
Let $\mathrm{M}$ be a domain, divided in two parts, $\mathrm{N}$ and $\mathrm{S}$, using a differentiable function $f$. Let $\mathrm{D}$ be the common border of $\mathrm{N}$ and $\mathrm{S}$. In $\mathrm{N}$ is defined a vector field $X$, and in $S$ a vector field $Y$, forming thereby a field $Z$, defined in $M$, called discontinuous vector field. Filippov [Fi] developed the rules for the transition of the orbits between $\mathrm{N}$ and $\mathrm{S}$, or their persistence in D. Sotomayor and Teixeira [S-T] used as domain the sphere $\mathrm{S}^{2}$ and developed the regularization method. This method consists in using a transition function $\varphi$ to define a one parameter family of continuous vector fields that approaches the discontinuous vector field, when the parameter goes to zero. These continuous vector fields are called regularized vector fields. Sotomayor and Teixeira provided conditions over $Z$, in order to the regularized vector fields be structuraly stable. This paper uses as domain a compact $M$ region contained in $\mathfrak{R}^{2}$. Using the regularization method, searches the conditions over $X$ and $Y$, in order to the regularized vector fields be structuraly stable. In this case was added the problem of the treatment of the points $\mathrm{p} \in \mathrm{D} \cap \partial \mathrm{M}$. It is proven, using translations and rotations, that the set of vetorial fields that satisfy these conditions is generic within the set of discontinuous vector fields. At the end, the bifurcations are studied, when some of the conditions for strutuctural stability are not fulfilled, specially the fields $\mathrm{X}$ and $\mathrm{Y}$ do not have singularities in $\mathrm{D}$.
\end{abstract}




\section{Índice}

Introdução

Capítulo 1

Campos Vetoriais de Andronov-Pontrjaguin-Peixoto e

Campos Vetoriais Descontínuos

1. Campos Vetoriais de Andronov-Pontrjaguin-Peixoto

2. Campos Vetoriais Descontínuos no Plano ........................... 3

3. Regularização de Campos Vetoriais Descontínuos ..................... 12

\section{Capítulo 2}

Campos Vetoriais Descontínuos no Plano

1. Regularização de Pontos Singulares e Poli-Trajetórias Fechadas ... 14

2. Regularização de Pontos Pertencentes à Intersecção da Curva D com $\partial \mathrm{M}$

3. Campos Vetoriais Descontínuos com Regularização de Andronov-Pontrjaguin-Peixoto

Capítulo 3

Introdução ao Estudo das Bifurcações de

Campos Vetoriais Descontínuos

1. Ponto Singular sobre a Curva D

2. Singularidade não Hiperbólica de $\mathrm{F}_{Z}$

Conclusões 


\section{Introdução}

Seja $A \subseteq \Re^{n}$ um conjunto aberto. Define-se um campo vetorial de classe $C^{k}, 1 \leq k \leq \infty$, como uma aplicação $X: A \rightarrow \Re^{n}$ de classe $C^{k}$. Ao campo vetorial $X$ pode ser associada a equação diferencial

$$
\mathrm{x}^{\prime}=\mathrm{X}(\mathrm{x})
$$

As soluções desta equação são chamadas trajetórias, órbitas ou curvas integrais de $\mathrm{X}$ ou da equação diferencial (1).

A equação diferencial (1) é na verdade um sistema de equações diferenciais autônomo (isto é, independente de t). Estudando-se a aplicação $X$ pode-se determinar alguns aspectos qualitativos importantes do retrato de fase deste sistema, sem que para isto seja necessário encontrar explicitamente a solução ou mesmo uma solução aproximada. Este tipo de abordagem de um sistema de equações diferenciais constitui o que é chamado de Teoria Qualitativa das Equações Diferenciais, e foi introduzido por Poincaré no final do século passado. Esta teoria é uma das principais bases para o estudo atual dos Sistemas Dinâmicos.

Em 1937 Andronov e Pontryaguin introduziram o conceito de estabilidade estrutural - um campo vetorial é estruturalmente estável se o seu retrato de fase resiste a pequenas perturbações, isto é, se o campo é equivalente através de um homeomorfismo a cada campo vetorial contido em uma de suas vizinhanças. Peixoto provou a densidade dos campos estruturalmente estáveis definidos em superfícies nos seus trabalhos de 1958 a 1962. Neste trabalho, será denominado conjunto de AndronovPontryaguin-Peixoto o conjunto de campos vetoriais contínuos que satisfaz as condições necessárias e suficientes para serem estruturalmente estáveis em regiões compactas do plano. Ver Capítulo 1, Definição 1.3.

A área de Sistemas Dinâmicos desenvolveu-se e hoje tem muitos ramos, por exemplo, o estudo das bifurcações - ocorre uma bifurcação numa família de campos vetoriais quando se verifica uma mudança brusca no retrato de fase dos elementos da família quando o parâmetro que rege a mesma cruza um certo valor, chamado ponto de bifurcação.

Mais recentemente, os Campos Vetoriais Descontínuos, outra subárea de Sistemas Dinâmicos vêm se desenvolvendo. Um sistema deste tipo é definido por uma aplicação $Z: \mathfrak{R}^{\mathrm{n}} \rightarrow \mathfrak{R}^{\mathrm{n}}$,

$$
x^{\prime}=Z(x),
$$

tal que $\mathrm{Z}$ é dado por duas expressões diferenciáveis $\mathrm{X}$ e $\mathrm{Y}$, distintas, definidas respectivamente em regiões $\mathrm{N}$ e $\mathrm{S}$ do espaço $\mathfrak{R}^{\mathrm{n}}$, com fronteira 
comum D, na qual Z pode ser considerado bi-valuado. Supõe-se que a hipersuperfície regular $\mathrm{D}$ e as regiões $\mathrm{N}$ e $\mathrm{S}$ podem ser definidas com o auxílio de uma função $f: \Re^{n} \rightarrow \Re$ de classe $C^{\infty}$ da seguinte forma

$$
\begin{aligned}
& \mathrm{D}=\left\{\mathrm{x} \in \mathfrak{R}^{\mathrm{n}} \mid f(\mathrm{x})=0\right\}, \\
& \mathrm{N}=\left\{\mathrm{x} \in \mathfrak{R}^{\mathrm{n}} \mid f(\mathrm{x}) \geq 0\right\} \mathrm{e} \\
& \mathrm{S}=\left\{\mathrm{x} \in \mathfrak{R}^{\mathrm{n}} \mid f(\mathrm{x}) \leq 0\right\} .
\end{aligned}
$$

Deste modo, pode-se re-escrever o sistema (2) na forma

$$
\mathrm{x}^{\prime}=\left(\begin{array}{l}
\mathrm{X}(\mathrm{x}), f(\mathrm{x}) \geq 0 \mathrm{e} \\
\mathrm{Y}(\mathrm{x}), f(\mathrm{x}) \leq 0 .
\end{array}\right.
$$

A dificuldade inicial no estudo destes sistemas é dar um significado ao que venha a ser uma órbita, uma vez que a teoria clássica de existência e unicidade de soluções, na ausência da continuidade e das condições de Lipschitz, não se aplica a eles. Ver [S1].

Dois livros clássicos sobre este assunto são: o de Andronov-Chaikin et al. [A-Ch] e o de Filippov [Fi]. Eles representam estágios distintos do desenvolvimento do estudo dinâmico dos sistemas descontínuos. O primeiro trata essencialmente de situações particulares provenientes de problemas com sistemas mecânicos e elétricos, mostrando a necessidade de uma fundamentação teórica para este tipo de sistemas e de uma teoria qualitativa que seja aplicável em problemas da Teoria de Oscilações. O segundo fornece uma fundamentação teórica e desenvolve, entre outras questões básicas, as regras (chamadas "convenções de Filippov") para a transição das órbitas entre as regiões $\mathrm{N}$ e $\mathrm{S}$, ou para a permanência das órbitas ao longo da hipersuperfície de descontinuidade D.

As definições de Filippov fornecem uma espécie de sistema dinâmico com seu respectivo Retrato de Fase, embora sem a unicidade de órbitas. Com ela formula-se uma Teoria de Estabilidade Estrutural por pequenas deformações de $Z$, ou seja, do par $(X, Y)$ em $(N, S)$. Filippov faz uma apresentação do trabalho de Koslova [K], que são formulados para regiões compactas do plano $(n=2)$ que encontram D transversalmente.

Mais recentemente, Sotomayor e Teixeira [S-T] introduziram o método de regularização que será utilizado nesta tese. A vantagem deste método é que um campo regularizado é um campo contínuo, para o qual pode-se aplicar toda a teoria clássica desenvolvida. Explicaremos resumidamente a seguir no que consiste este método. 
Uma função $\varphi: \Re \rightarrow \Re, \varphi$ de classe $C^{\infty}$, é uma função de transição se

$$
\begin{aligned}
& \varphi(t)=\left(\begin{array}{l}
0, t \in(-\infty,-1] \text { e } \\
1, t \in[1, \infty),
\end{array}\right. \\
& \varphi^{\prime}(t)>0 \text { se } t \in(-1,1) .
\end{aligned}
$$

Define-se $\varphi_{\varepsilon}(t)=\varphi(t / \varepsilon)$.

O método de regularização consiste na aproximação de sistemas descontínuos da forma (3) por uma família a um parâmetro de sistemas diferenciáveis, definida com o auxílio de uma função de transição. Uma $\varphi_{\varepsilon}$ regularização do sistema (3) é dada por

$$
Z_{\varepsilon}(\mathrm{q})=\left(1-\varphi_{\varepsilon}(f(\mathrm{q}))\right) Y(\mathrm{q})+\varphi_{\varepsilon}(f(\mathrm{q})) X(\mathrm{q}),
$$

onde $q \in \mathfrak{R}^{\mathrm{n}}$ e o parâmetro $\varepsilon$ é sempre estritamente positivo.

Observa-se que há uma região em $\mathfrak{R}^{\mathrm{n}}$ contendo a hipersuperfície $\mathrm{D}$ onde o campo $Z_{\varepsilon}$ é um promédio dos campos $\mathrm{X}$ e Y. Esta região é dada pelos pontos $\mathrm{q} \in \mathfrak{R}^{\mathrm{n}}$ tais que $\varphi_{\varepsilon}(f(\mathrm{q})) \in(0,1)$ e é chamada de faixa de regularização.

A linha de trabalho seguida por Filippov em [Fi] é diferente da seguida por Sotomayor e Teixeira e nesta tese. Filippov deu continuidade ao trabalho de Koslova $[\mathrm{K}]$, utilizando domínios $\mathrm{G}$ contidos em $\mathfrak{R}^{\mathrm{n}}$, divididos em um número finito de subdomínios $G_{i}$ 's, em cada um dos quais o campo vetorial é contínuo até a fronteira. Estabeleceu as convenções de Filippov, que trata da transição das trajetórias através das fronteiras dos subdomínios $\mathrm{G}_{\mathrm{i}}$ 's, ou da permanência das mesmas na fronteira. Nesta tese estas definições foram utilizadas. Filippov fez uma classificação das singularidades que surgem na fronteira para o caso bi-dimensional. No livro de Filippov também se encontram uma extensão do conceito de estabilidade estrutural para os sistemas descontínuos e as condições necessárias e suficientes para a estabilidade estrutural de um sistema descontínuo.

Em [S-T], Sotomayor e Teixeira trabalharam com campos vetoriais definidos numa esfera bi-dimensional. Nesta esfera foi definido o campo vetorial descontínuo $Z$, conforme descrito no início desta introdução. Desenvolveram o método da regularização, também já explicado e estabeleceram condições para que a regularização de um campo vetorial descontínuo forneça campos vetoriais de Andronov-Pontrjaguin-Peixoto, para $\varepsilon$ pequeno. 
Nesta tese, seguindo-se a linha de Sotomayor e Teixeira, foram estudados os campos descontínuos $\mathrm{Z}$ definidos em regiões $\mathrm{M}$ como abaixo.

Seja $M \subset \mathfrak{R}^{2}$ uma região compacta, conexa e com fronteira diferenciável. Pode-se considerar que $M$ é definida com o auxílio de uma função $\beta: \mathfrak{R}^{2} \rightarrow \mathfrak{R}, \beta$ de classe $C^{\infty}, \mathrm{v}=0$ valor regular de $\beta$, com

$$
\begin{aligned}
& M=\{x \mid \beta(x) \geq 0\} e \\
& \partial M=\{x \mid \beta(x)=0\} .
\end{aligned}
$$

Nos seus trabalhos, Filippov, Sotomayor e Teixeira utilizaram domínios sem fronteira. Nesta tese foi acrescentado um problema que não havia anteriormente, que é o de saber o que ocorre em vizinhanças de

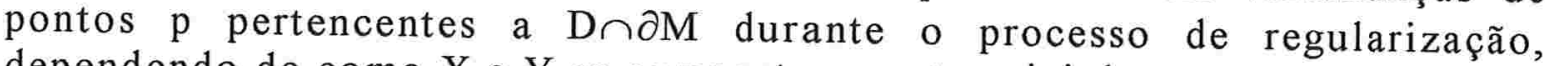
dependendo de como X e Y se comportam nestas vizinhanças.

Neste trabalho identificam-se condições sobre os campos X e Y para que, dada uma função de transição $\varphi$, exista $\varepsilon_{0}>0$ tal que para $0<\varepsilon<\varepsilon_{0}$, o campo vetorial regularizado $Z_{\varepsilon}$ seja estruturalmente estável. Como é sabido, para que isto ocorra $Z_{\varepsilon}$ deve ser um campo vetorial de AndronovPontrjaguin-Peixoto (A-P-P). Por isto, o trabalho é desenvolvido investigando-se as condições sobre $\mathrm{X}$ e $\mathrm{Y}$ para que $Z_{\varepsilon}$ possua todas as suas singularidades hiperbólicas e no interior de $M$; todas as suas órbitas periódicas hiperbólicas e no interior de $M$; as tangências de $Z_{\varepsilon}$ com $\partial \mathrm{M}$ sejam no máximo quadráticas e $Z_{\varepsilon}$ não possua conexão de separatrizes. Neste trabalho também é provado um Teorema de Genericidade, ou seja, que o conjunto $G^{r}$ de campos vetoriais descontínuos que possuem as características desejadas é aberto e denso dentro do conjunto dos campos vetoriais descontínuos em geral. Depois disto é iniciado o estudo das bifurcações, utilizando-se famílias de campos vetoriais descontínuos $Z_{\lambda}=\left(X_{\lambda}, Y_{\lambda}\right)$ tal que, para $\lambda=0$, o campo vetorial descontínuo $Z_{0}$ não pertence ao conjunto $\mathrm{G}^{\mathrm{r}}$. As consequências deste fato para a família de campos vetoriais regularizados $Z_{\lambda, \varepsilon}$ são estudadas para duas situações: o campo $\mathrm{X}_{0}$ possui uma singularidade hiperbólica sobre a curva $\mathrm{D}$ (esta situação gera seis casos); o campo de Filippov $F_{Z}$ (ver Capítulo 1, Definição 2.3) possui uma singularidade não hiperbólica.

A tese está dividida da seguinte maneira:

No Capítulo 1 são relembrados os conceitos e teoremas a respeito dos campos vetoriais de Andronov-Pontrjaguin-Peixoto. Também são formuladas as definições básicas sobre os campos vetoriais descontínuos e o método da regularização. 
No Capítulo 2 são tratadas as questões relativas a pontos singulares e órbitas periódicas que surgem em $Z_{\varepsilon}$ em decorrência da regularização. Também são estudadas a aparição de tangências e a conexão de separatrizes. Com base nos resultados então obtidos é definido um conjunto de campos vetoriais descontínuos que possui a propriedade da regularização de cada campo deste conjunto fornecer uma família de campos vetoriais de Andronov-Pontrjaguin-Peixoto. Também é feita a prova que este conjunto é aberto e denso dentro do conjunto dos campos vetoriais descontínuos. Nos pontos pertinentes deste Capítulo serão indicados os aprimoramentos e complementos à análise de Sotomayor e Teixeira [S-T] aqui desenvolidos.

No Capítulo 3 é iniciado o estudo das bifurcações dos campos vetoriais descontínuos. Neste sentido, é estudado o efeito que uma singularidade sobre a curva de descontinuidade causa na família de campos regularizados. Observa-se que em alguns casos é possível que a família de campos regularizados seja de Andronov-Pontrjaguin-Peixoto, ainda que o campo vetorial descontínuo não esteja no conjunto genérico definido no Capítulo 2. Também é estudada a consequência do campo $F_{Z}$ possuir uma singularidade não hiperbólica para a família de campos vetoriais regularizados. 


\title{
Capítulo 1 \\ Campos Vetoriais de Andronov-Pontrjaguin- Peixoto e Campos Vetoriais Descontínuos
}

\author{
Este capítulo é introdutório ao estudo dos Campos Vetoriais \\ Descontínuos.
}

São definidos conceitos básicos e enunciados alguns resultados sobre os Campos Vetoriais de Andronov-Pontrjaguin-Peixoto (A-P-P). É um fato bastante divulgado que estes campos são genéricos em $\mathfrak{R}^{2}$. Como um dos objetivos desta tese é caracterizar os Campos Vetoriais Descontínuos que possuam propriedades genéricas, os Campos Vetoriais de AndronovPontrjaguin-Peixoto são o ponto de partida natural.

Em seguida serão apresentados os Campos Vetoriais Descontínuos, as convenções de Filippov e o método da regularização desenvolvido por Sotomayor e Teixeira em [S-T].

\section{Campos Vetoriais de Andronov-Pontrjaguin-Peixoto}

Nesta seção serão enunciados algumas definições e resultados sobre campos vetoriais, em particular, sobre os Campos Vetoriais de AndronovPontrjaguin-Peixoto.

Seja $M$ uma região bi-dimensional com bordo suave contida em $\mathfrak{R}^{2}$, compacta e conexa, dada por uma função $\beta: \Re^{2} \rightarrow \Re$ de classe $C^{\infty}$, tal que $v=0$ é valor regular de $\beta, M=\{(x, y): \beta(x, y) \geq 0\}$ e $\partial M=\left\{\beta^{-1}(0)\right\}$.

Denota-se o conjunto dos campos vetoriais de classe $C^{\mathrm{r}}$ definidos em $\mathrm{M}$ por $\mathfrak{X}^{\mathrm{r}}(\mathrm{M})$. Considera-se este conjunto munido da norma $C^{\mathrm{r}}$, isto é, $|X|_{r}=\sup \left\{|X(p)|+|D X(p)|+\ldots+\left|D^{r} X(p)\right|, p \in M\right\}$, onde $D^{i} X(p)$ denota a i-ésima derivada de $X$ em $p$ e $\left|D^{i} X(p)\right|$ denota a sua norma como função i-linear simétrica de $\left(\mathfrak{R}^{2}\right)^{\mathrm{i}}$ em $\mathfrak{R}^{2}$.

\subsection{Definição}

Dois campos vetoriais $\mathrm{X}$ e $\mathrm{Y} \in \mathbb{X}^{\mathrm{r}}(\mathrm{M})$ são ditos topologicamente equivalentes se existe um homeomorfismo que leva órbita de $\mathrm{X}$ em órbita de $\mathrm{Y}$, preservando a orientação. 
Um campo vetorial $\mathrm{X} \in \mathcal{K}^{\mathrm{r}}(\mathrm{M})$ é dito um campo vetorial estruturalmente estável se for topologicamente equivalente a todos os campos vetoriais contidos em uma de suas vizinhanças.

Denota-se o conjunto dos campos vetoriais de classe $C^{\text {r }}$ estruturalmente estáveis definidos em $M$ por $\mathrm{S}^{\mathrm{r}}(\mathrm{M})$.

Os Teoremas de Andronov-Pontrjaguin-Peixoto, que serão enunciados a seguir, fornecem a caracterização e a genericidade dos campos estruturalmente estáveis definidos em regiões compactas do plano.

\subsection{Definição}

Denota-se por $\Sigma^{\mathrm{r}}(\mathrm{i}), \mathrm{i}=1, \ldots, 4$, os subconjuntos de $\mathfrak{X}^{\mathrm{r}}(\mathrm{M})$ definidos da seguinte maneira:

$\Sigma^{\mathrm{r}}(1)=\left\{\mathrm{X} \in \mathfrak{K}^{\mathrm{r}}(\mathrm{M}) \mid\right.$ todos os pontos singulares de $\mathrm{X}$ são hiperbólicos $\mathrm{e}$ estão contidos no interior de $M\}$.

$\Sigma^{\mathrm{r}}(2)=\left\{\mathrm{X} \in \mathfrak{X}^{\mathrm{r}}(\mathrm{M}) \mid\right.$ todas as órbitas periódicas de $\mathrm{X}$ são hiperbólicas e estão contidas no interior de $\mathrm{M}\}$.

$\Sigma^{\mathrm{r}}(3)=\left\{\mathrm{X} \in \mathscr{X}^{\mathrm{r}}(\mathrm{M}) \mid\right.$ para todo ponto $\mathrm{p} \in \partial \mathrm{M}$, a órbita de $\mathrm{X}$ por $\mathrm{p}, \varphi(\mathrm{t}, \mathrm{p})$, é transversal a $\partial \mathrm{M}$ (isto é, $(\partial / \partial \mathrm{t})(\beta \circ \varphi)(0, \mathrm{p}) \neq 0)$ ou tem contato parabólico com $\partial \mathrm{M}$ (isto é, $(\partial / \partial \mathrm{t})\left(\beta_{\circ} \varphi\right)(0, \mathrm{p})=0$ e $\left.\left.\left(\partial^{2} / \partial \mathrm{t}^{2}\right)\left(\beta_{\circ} \varphi\right)(0, \mathrm{p}) \neq 0\right)\right\}$.

$\Sigma^{\mathrm{r}}(4)=\left\{\mathrm{X} \in 犬^{\mathrm{r}}(\mathrm{M}) \mid\right.$ o campo vetorial $\mathrm{X}$ não possui conexões $\mathrm{de}$ separatrizes de selas, de separatrizes de selas com separatrizes de tangência ou de separatrizes de tangências\}.

\subsection{Definị̧ão}

Define-se o conjunto dos campos vetoriais de Andronov-PontrjaguinPeixoto $(A-P-P)$ de classe $C^{r}$ em $M$ por $\Sigma^{r}(M)=\cap \Sigma^{r}(i), i=1, \ldots, 4$.

\subsection{Teorema}

$O$ conjunto $\Sigma^{r}(M)$ é aberto e denso em $\mathfrak{X}^{r}(M), r \geq I$.

\subsection{Teorema}

Seja $X \in \mathscr{X}^{r}(M)$. Então $X \in S^{r}(M)$ se e só se $X \in \Sigma^{r}(M)$, isto é, $\Sigma^{r}(M)=S^{r}(M), r \geq 1$. 
A demonstração destes dois teoremas pode ser encontrada em [S2]. Observa-se que a densidade é demonstrada utilizando-se conceitos de medida de Lebesgue, como será explicado no final do Capítulo 2, ocasião em que será demonstrado um teorema de densidade para o caso descontínuo, utilizando-se os mesmos conceitos.

Outro teorema importante que será utilizado neste trabalho é o Teorema de Poincaré-Bendixson:

\subsection{Teorema de Poincaré-Bendixson}

Sejam $X \in \mathcal{X}^{r}(M), r \geq 1, e \psi(t)=\psi(t, p)$ uma curva integral de $X$, definida para todo $t \geq 0$, tal que $\gamma_{p}^{+}=\{\psi(t, p) / t \geq 0\}$ esteja contida num compacto $K \subset \operatorname{Int}(M)$. Suponha que o campo $X$ possua um número finito de singularidades em $\omega(p)$, o $\omega$-limite de $p$. Têm-se as seguintes alternativas:

a) Se $\omega(p)$ contém somente pontos regulares, então $\omega(p)$ é uma órbita periódica;

b) Se $\omega(p)$ contém pontos regulares e singulares, então $\omega(p)$ consiste de um conjunto de órbitas, cada um dos quais tende a um destes pontos singulares quando $t \rightarrow \pm \infty$;

c) Se $\omega(p)$ não contém pontos regulares, então $\omega(p)$ é um ponto singular.

A demonstração deste teorema pode ser encontrada em [S1].

\section{Campos Vetoriais Descontínuos no Plano}

Esta seção versa sobre os Campos Vetoriais Descontínuos, que são o principal objeto de estudo deste trabalho. Preliminarmente, serão formuladas algumas definições.

Seja $f: \mathrm{M} \rightarrow \mathfrak{R}$ uma função de classe $C^{\infty}$ tendo $\mathrm{v}=0$ como valor regular e seja $\mathrm{D}=\left\{f^{-1}(0)\right\}$. Será suposto que $\mathrm{D}$ possui uma componente conexa e $\mathrm{M}$ - $\mathrm{D}$ duas componentes conexas, dadas por $\mathrm{N}=\left\{f^{-1}(0,+\infty)\right\}$ e $\mathrm{S}=\left\{f^{-1}(-\infty, 0)\right\}$. Esta suposição não influi nos resultados, sendo apenas para simplificação das demonstrações.

\subsection{Definição}

Dados $\mathrm{X}$ e $\mathrm{Y} \in \mathcal{*}^{\mathrm{r}}(\mathrm{M}), \mathrm{r} \geq 1$, e uma função $f$ como acima, define-se o campo vetorial descontínuo $Z=(X, Y)$ da seguinte maneira:

$$
Z(q)=\left\{\begin{array}{l}
X(q), \text { se } f(q) \geq 0, \\
Y(q), \text { se } f(q) \leq 0
\end{array}\right.
$$


Denota-se por $\Omega^{r}(M, f)$ o espaço de campos vetoriais descontínuos de classe $\mathrm{C}^{\mathrm{r}}$ definidos em $\mathrm{M}$ com o auxílio da função $f$.

Observa-se que o campo $Z$ é em geral bi-valuado nos pontos da curva $\mathrm{D}$, a qual é chamada de curva de descontinuidade.

Da hipótese de $\mathrm{D}$ só possuir uma componente conexa, segue que existem apenas dois casos a serem considerados:

- Quando $\partial \mathrm{D}=\mathrm{D} \cap \partial \mathrm{M} \neq \phi$;

Neste caso, acrescenta-se a hipótese que a intersecção de D com $\partial \mathrm{M}$ é transversal, fato que pode ser expresso por $\nabla f \wedge \nabla \beta \neq 0$ em $\partial \mathrm{D}$.

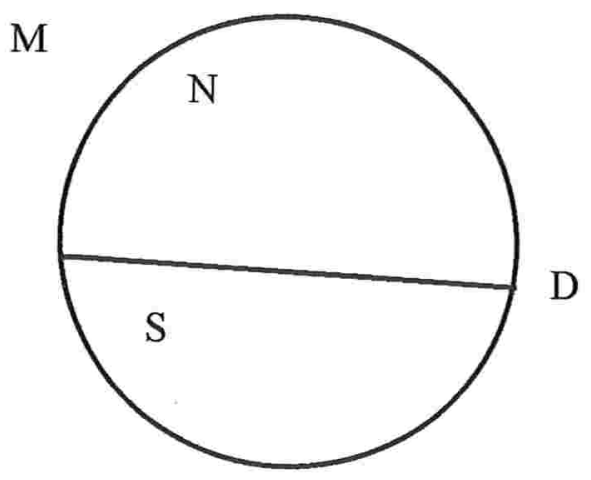

Fig. 1

- Quando $\partial \mathrm{D}=\mathrm{D} \cap \partial \mathrm{M}=\phi$.

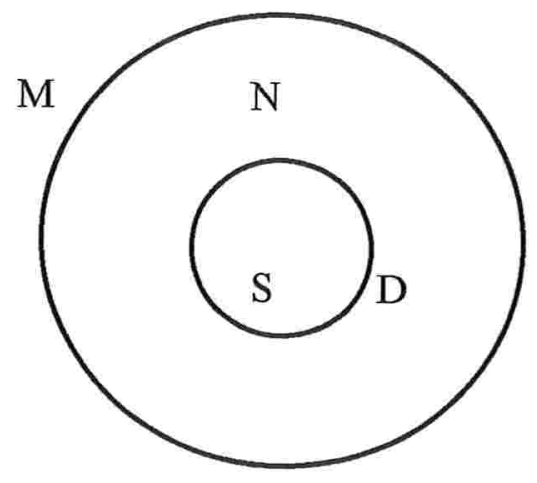

Fig. 2

Para o desenvolvimento deste trabalho, é necessário que se estude o comportamento do campo descontínuo $Z=(X, Y)$ na vizinhança da curva $D$. Para isto, serão dadas a seguir algumas definições úteis. 


\subsection{Definição}

Dado $Z \in \Omega^{\mathrm{r}}(\mathrm{M}, f), \mathrm{r} \geq 1$, seguindo a terminologia de Filippov [Fi], genericamente distingue-se os seguintes tipos de pontos possíveis em D:

Pontos de Costura: $\mathrm{p} \in \mathrm{D}$ tal que $(\mathrm{X} f)(\mathrm{p})(\mathrm{Y} f)(\mathrm{p})>0$, onde $\mathrm{X} f=\mathrm{X} \bullet \nabla f$.
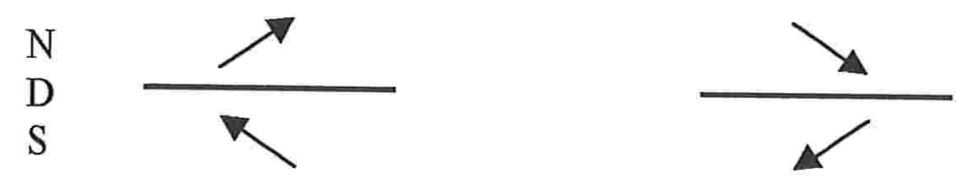

Fig. 3

Pontos de Escape: $\mathrm{p} \in \mathrm{D}$ tal que $\mathrm{X} f(\mathrm{p})>0$ e $\mathrm{Y} f(\mathrm{p})<0$.

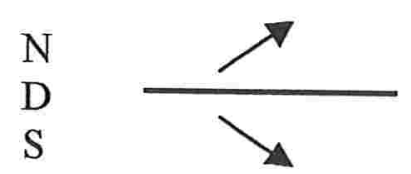

Fig. 4

Pontos de Deslizamento: $\mathrm{p} \in \mathrm{D}$ tal que $\mathrm{X} f(\mathrm{p})<0$ e $\mathrm{Y} f(\mathrm{p})>0$.

N
D
S

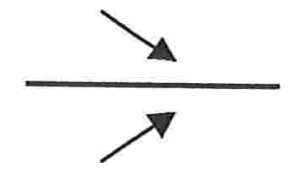

Fig. 5

Pela definição destas classes de pontos, é imediato que eles ocorrem em abertos de $\mathrm{D}$, definindo então arcos de pontos do mesmo tipo, a saber, arcos de costura (COS), arcos de deslizamento (DES) e arcos de escape (ESC).

\subsection{Definição}

Define-se um campo auxiliar $\mathrm{F}_{Z}$, dito campo de Filippov, nos arcos DES e ESC de D da seguinte maneira:

Seja $p \in$ DES ou ESC, então $F_{Z}(p)$ denota o vetor, tangente a $D$, contido no cone gerado por $\mathrm{X}(\mathrm{p})$ e $\mathrm{Y}(\mathrm{p})$.

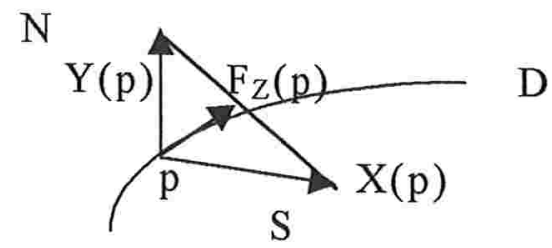

Fig. 6 


\subsection{Notação}

No que segue, a menos de menção em contrário, será utilizada a seguinte notação:

Sejam $Z=(X, Y) \in \Omega^{r}(M, f)$ e $p \in D$. Localmente, em torno de uma vizinhança de $p$, pode-se considerar coordenadas locais tais que $D=\{y=0\}$, $f(\mathrm{x}, \mathrm{y})=\mathrm{y}, \mathrm{p}=(0,0), \mathrm{X}(\mathrm{x}, \mathrm{y})=(\mathrm{a}(\mathrm{x}, \mathrm{y}), \mathrm{b}(\mathrm{x}, \mathrm{y}))$ e $\mathrm{Y}(\mathrm{x}, \mathrm{y})=(\mathrm{c}(\mathrm{x}, \mathrm{y}), \mathrm{d}(\mathrm{x}, \mathrm{y}))$.

Se o ponto $p$ considerado pertencer a $\partial M$, isto é, $p \in D \cap \partial M$, as coordenadas podem ser tomadas de modo que tenha-se também $\beta(x, y)=x$ e $\partial \mathrm{M}=\{\mathrm{x}=0\}$, uma vez que $\mathrm{D}$ e $\partial \mathrm{M}$ se encontram transversalmente, por hipótese.

\subsection{Lema}

Seja $p \in$ DES ou ESC. Na Notação 2.4, tem-se $p=0 e$

$$
F_{Z}(0)=\left[a(0)-b(0) \frac{((c(0)-a(0))}{(d(0)-b(0))}, 0\right] \text {. }
$$

demonstração

Pode-se supor, sem perda de generalidade, que $p \in D E S$.

Seja $r$ a reta que passa por $(a(0), b(0))$ e $(c(0), d(0))$.

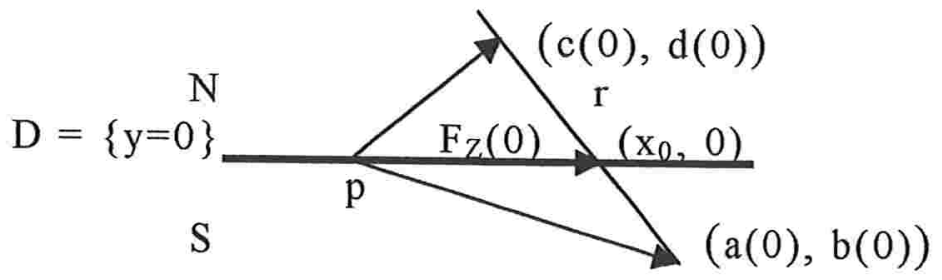

Fig. 7

Como a curva de descontinuidade coincide localmente com o eixo $\mathrm{x}$, tem-se $\mathrm{F}_{Z}(0)=\left(\mathrm{x}_{0}, 0\right)$, onde $\left(\mathrm{x}_{0}, 0\right) \in r$, logo basta encontrar a equação que define a reta $r$ e o ponto em que a mesma cruza o eixo $\mathrm{x}$.

A inclinação da reta $r$ é dada por $m=(d(0)-b(0)) /(c(0)-a(0))$.

Então a equação que define a reta $r$ é:

$y=((d(0)-b(0)) /(c(0)-a(0)))(x-a(0))+b(0)$ 
Tomando-se $y=0$, tem-se

$$
\begin{aligned}
& 0=((\mathrm{d}(0)-\mathrm{b}(0)) /(\mathrm{c}(0)-\mathrm{a}(0)))(\mathrm{x}-\mathrm{a}(0))+\mathrm{b}(0) \\
& \Rightarrow-\mathrm{b}(0)((\mathrm{c}(0)-\mathrm{a}(0)) /(\mathrm{d}(0)-\mathrm{b}(0)))=\mathrm{x}-\mathrm{a}(0) \\
& \Rightarrow \mathrm{x}=\mathrm{a}(0)-\mathrm{b}(0)((\mathrm{c}(0)-\mathrm{a}(0)) /(\mathrm{d}(0)-\mathrm{b}(0))) .
\end{aligned}
$$$$
\text { Logo, } \mathrm{F}_{Z}(0)=(\mathrm{a}(0)-\mathrm{b}(0)((\mathrm{c}(0)-\mathrm{a}(0)) /(\mathrm{d}(0)-\mathrm{b}(0))), 0) \text {. }
$$

Da definição de $F_{Z}$, é imediato que esta fórmula também é válida quando p pertence ao arco ESC.

Os pontos D-regulares e as D-dobras, definidos a seguir, serão os pontos da curva $\mathrm{D}$ que possuirão vizinhanças nas quais não surgirão pontos singulares durante o processo de regularização. Este fato será provado no Capítulo 2.

\subsection{Definição}

Um ponto $\mathrm{p} \in \mathrm{D}$ é dito um ponto D-regular de $Z$ se uma das seguintes condições é satisfeita:

1) $\mathrm{p} \in \operatorname{COS}$ (isto é, $\mathrm{X} f(\mathrm{p}) \mathrm{Y} f(\mathrm{p})>0$ ), ou

2) $\mathrm{p} \in \mathrm{DES}$ ou $\operatorname{ESC}$ (isto é, $\mathrm{X} f(\mathrm{p}) \mathrm{Y} f(\mathrm{p})<0$ ), $\operatorname{mas} \operatorname{det}[\mathrm{X}, \mathrm{Y}](\mathrm{p}) \neq 0$ (isto é, os vetores $X(p)$ e $Y(p)$ são linearmente independentes, o que implica que p não é um ponto crítico de $F_{Z}$, como se verá na Definição 2.8).

\subsection{Definição}

Um ponto $\mathrm{p} \in \mathrm{D}$ é dito uma D-dobra se é um ponto de contato quadrático de $\mathrm{X}$ com $\mathrm{D}$ (isto é, $\mathrm{Y} f(\mathrm{p}) \neq 0, \mathrm{X} f(\mathrm{p})=0$ e $\mathrm{X}^{2} f(\mathrm{p}) \neq 0$ ), ou de $\mathrm{Y}$ com D (isto é, $\mathrm{X} f(\mathrm{p}) \neq 0, \mathrm{Y} f(\mathrm{p})=0$ e $\mathrm{Y}^{2} f(\mathrm{p}) \neq 0$ ).

As figuras abaixo são exemplos de D-dobras.

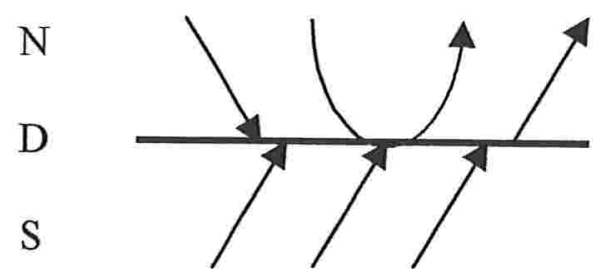

Fig. 8

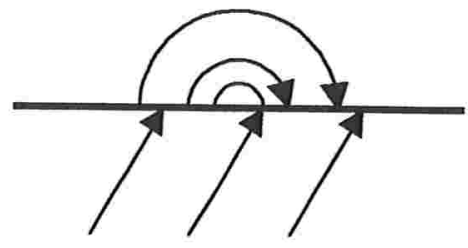

Fig. 9 
Os pontos críticos hiperbólicos de $\mathrm{F}_{Z}$, definidos a seguir, serão os pontos da curva $\mathrm{D}$ que gerarão uma única singularidade hiperbólica em cada campo regularizado, como será visto no Capítulo 2.

\subsection{Definição}

Um ponto $\mathrm{p} \in \mathrm{D}$ é dito um ponto critico de $F_{Z}$ se $\mathrm{X} f(\mathrm{p}) \mathrm{Y} f(\mathrm{p})<0$ (isto é, $\mathrm{p} \in \mathrm{DES}$ ou $\mathrm{p} \in \mathrm{ESC}) \mathrm{e} \operatorname{det}[\mathrm{X}, \mathrm{Y}](\mathrm{p})=0$. hiperbólico.

Se também valer $\mathrm{d}\left(\left.\operatorname{det}[\mathrm{X}, \mathrm{Y}]\right|_{\mathrm{D}}\right)(\mathrm{p}) \neq 0, \mathrm{p}$ será dito ponto crítico

As figuras abaixo são exemplos de pontos críticos hiperbólicos do campo $\mathrm{F}_{Z}$.

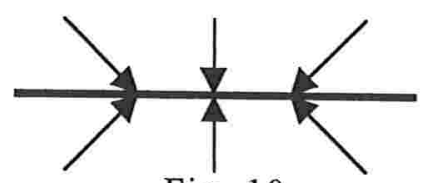

Fig. 10

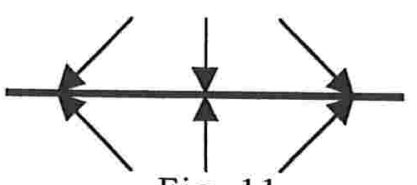

Fig. 11

\subsection{Definição}

Seja $\mathrm{p}$ um ponto crítico hiperbólico de $\mathrm{F}_{Z}$.

Diz-se que p é do tipo sela se $\mathrm{p} \in \mathrm{DES}$ e $\mathrm{p}$ é um repulsor de $\mathrm{F}_{Z}$ (isto é, $\mathrm{d}\left(\left.\operatorname{det}[\mathrm{X}, \mathrm{Y}]\right|_{\mathrm{D}}\right)(\mathrm{p})>0$ ), ou $\mathrm{p} \in \mathrm{ESC}$ e $\mathrm{p}$ é um atrator de $\mathrm{F}_{\mathrm{Z}}$ (isto é, $\left.\mathrm{d}\left(\left.\operatorname{det}[\mathrm{X}, \mathrm{Y}]\right|_{\mathrm{D}}\right)(\mathrm{p})<0\right)$.

As figuras seguintes representam estas duas situações:

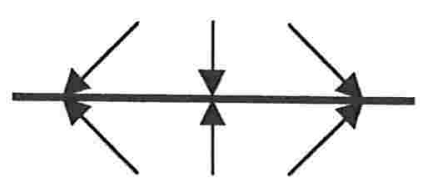

Fig. 12

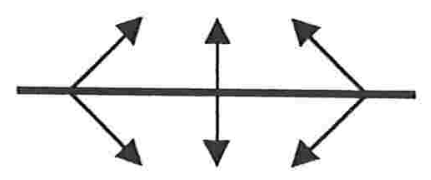

Fig. 13

Diz-se que p é do tipo nó se $\mathrm{p} \in \mathrm{DES}$ e p é um atrator de $\mathrm{F}_{Z}$ (isto é, $\left.\mathrm{d}\left(\left.\operatorname{det}[\mathrm{X}, \mathrm{Y}]\right|_{\mathrm{D}}\right)(\mathrm{p})<0\right)$, ou $\mathrm{p} \in \mathrm{ESC}$ e $\mathrm{p}$ é um repulsor de $\mathrm{F}_{Z}$ (isto é, $\mathrm{d}(\operatorname{det}[\mathrm{X}, \mathrm{Y}] \mid \mathrm{D})(\mathrm{p})>0)$.

As duas situações estão ilustradas nas figuras seguintes:

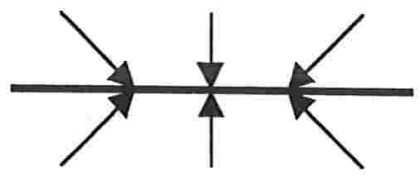

Fig. 14

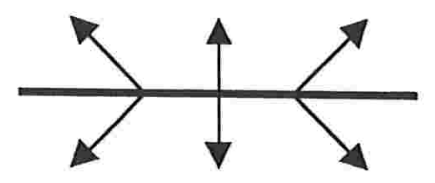

Fig. 15 


\subsection{Definição}

Um ponto $\mathrm{p} \in \mathrm{D}$ é dito uma $D$-singularidade elementar de $Z$ se

(i) p é uma D-dobra de $Z$; ou

(ii) $\mathrm{p}$ é um ponto crítico hiperbólico de $\mathrm{F}_{\mathrm{Z}}$.

\subsection{Definição}

Uma separatriz de $Z$ é um arco de trajetória de $\mathrm{X}$ ou $\mathrm{Y}$ que tangencia $\partial \mathrm{M}$ ou um arco de trajetória de $\mathrm{X}, \mathrm{Y}$ ou $\mathrm{F}_{Z}$ cujo $\alpha$ ou $\omega$-limite é um ponto sela de $\mathrm{X}, \mathrm{Y}$ ou $\mathrm{F}_{Z}$.

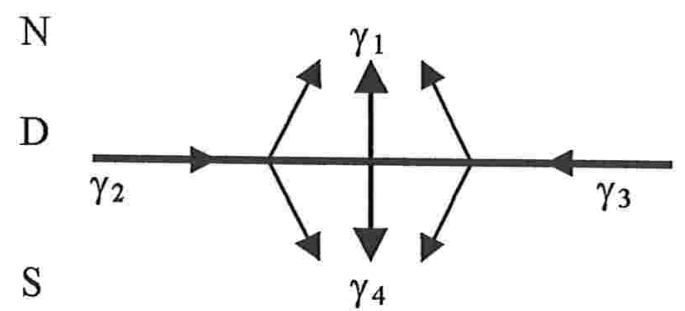

Fig. 16

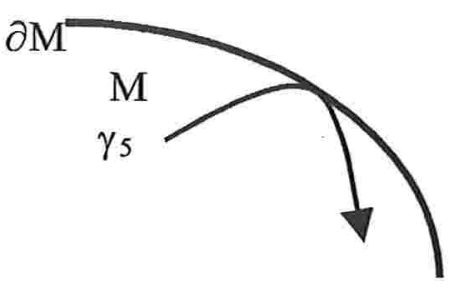

Fig. 17 do campo $Z$.

Nas figuras 16 e 17 , os $\operatorname{arcos} \gamma_{1}, \ldots, \gamma_{5}$ são exemplos de separatrizes

Uma separatriz dupla é uma trajetória de $\mathrm{X}$, de $\mathrm{Y}$ ou de $\mathrm{F}_{Z}$ cujos $\alpha$ e $\omega$-limites são selas, ou uma separatriz de sela de $\mathrm{X}$ ou de $\mathrm{Y}$ que tangencia $\partial \mathrm{M}$ ou ainda uma trajetória de $\mathrm{X}$ ou de $\mathrm{Y}$ que tangencia $\partial \mathrm{M}$ duas vezes.

\subsection{Definição}

Uma curva $\gamma$ composta por arcos regulares de trajetórias de $\mathrm{X}$ em N, e/ou de $\mathrm{Y}$ em $\mathrm{S}$ e/ou de $\mathrm{F}_{\mathrm{z}}$ em D é chamada uma poli-trajetória se:

(i) $\quad \gamma$ contém arcos de pelo menos dois entre os campos $\mathrm{X}, \mathrm{Y}$ e $\mathrm{F}_{Z}$ ou é composta por um arco de $\mathrm{F}_{\mathrm{Z}}$;

(ii) a transição de $\operatorname{arcos}$ de trajetória de $\mathrm{X}$ para $\operatorname{arcos}$ de $\mathrm{Y}$ é feita em pontos de costura;

(iii) a transição de $\operatorname{arcos}$ de trajetória de $\mathrm{X}$ ou de $\mathrm{Y}$ para $\operatorname{arcos}$ de $\mathrm{F}_{\mathrm{z}}$ é feita através de $\mathrm{D}$-dobras ou de pontos regulares do arco de escape ou do arco deslizante, respeitando-se o sentido dos arcos de trajetória. 


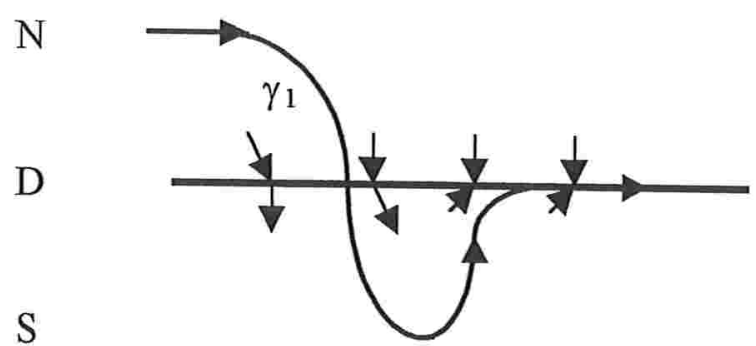

Fig. 18

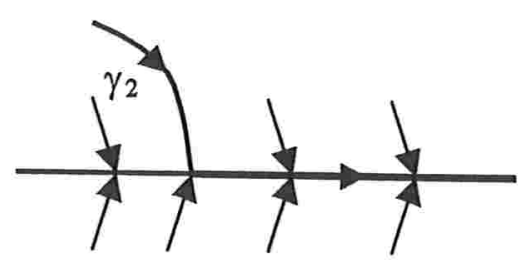

Fig. 19

Nas figuras acima, $\gamma_{1}$ e $\gamma_{2}$ são exemplos de poli-trajetórias.

\subsection{Observação}

É importante observar que os arcos de $\mathrm{F}_{Z}$ pertencem a infinitas politrajetórias.

\subsection{Definição}

Uma conexão de separatrizes de $Z$ é uma separatriz dupla ou uma poli-trajetória $\gamma$ que possui na sua composição uma separatriz dupla ou duas separatrizes.

$\mathrm{N}$

D

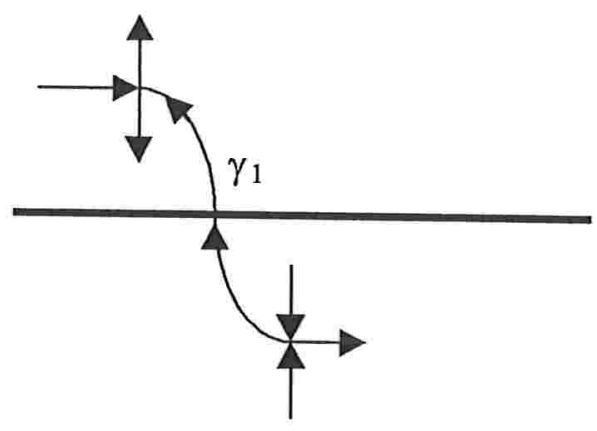

Fig. 20

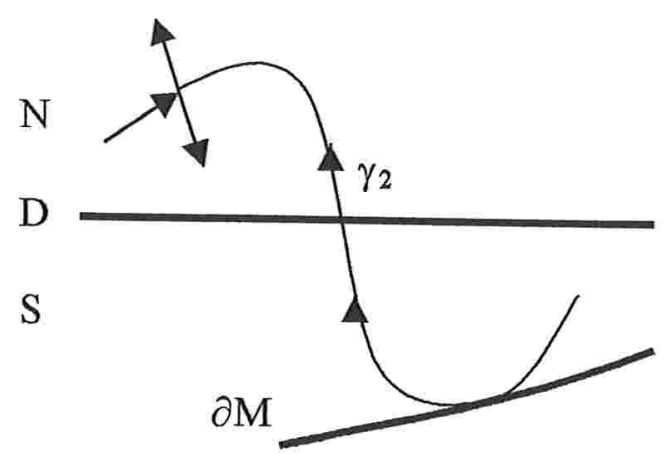

Fig. 21

As figuras acima são exemplos de conexões de separatrizes de um campo vetorial descontínuo $Z$.

\subsection{Definição}

Seja $\gamma$ uma poli-trajetória fechada de $Z=(X, Y)$.

Diz-se que $\gamma$ é uma poli-trajetória fechada tipo $I$ se $\gamma$ encontra D somente em pontos de costura.

Diz-se que $\gamma$ é uma poli-trajetória fechada tipo II se $\gamma=\mathrm{D}$. 
Diz-se que $\gamma$ é uma poli-trajetória fechada tipo III se $\gamma$ contém pelo menos uma D-dobra de $\mathrm{Z}$.

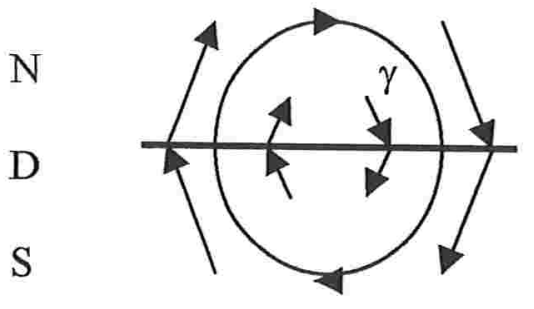

Poli-Trajetória Fechada Tipo I

Fig. 22

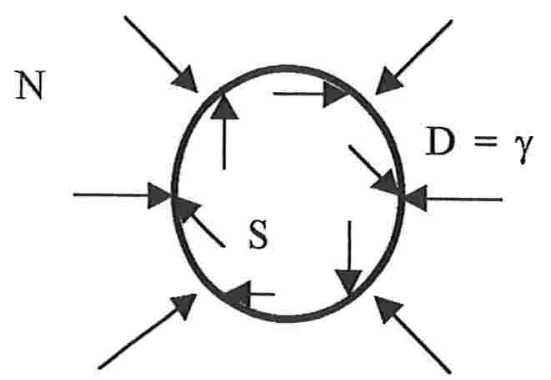

Poli-Trajetória Fechada Tipo II

Fig. 24

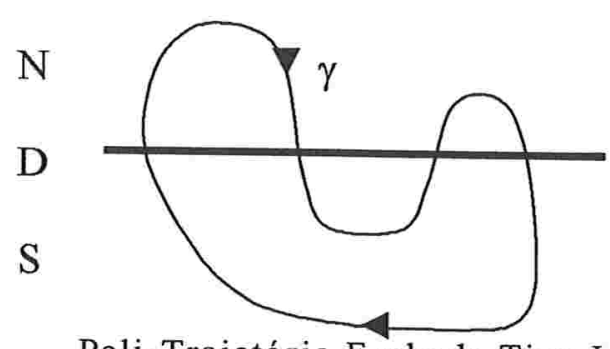

Poli-Trajetória Fechada Tipo I Fig. 23

$\mathrm{N}$

D

S

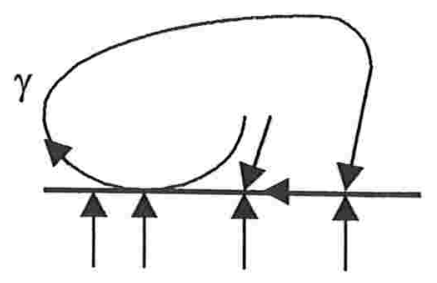

Poli-Trajetória Fechada Tipo III Fig. 25

\subsection{Definição}

Dada uma poli-trajetória fechada tipo I $\gamma=\gamma_{0} \cup \gamma_{1} \cup \ldots \cup \gamma_{k}$, onde $\gamma_{2 i} \in \mathrm{N}$ e $\gamma_{2 \mathrm{i}+1} \in \mathrm{S}$, para $\mathrm{i}=0, \ldots,(\mathrm{k}-1) / 2 ; \gamma_{\mathrm{j}} \cap \mathrm{D}=\left\{\mathrm{p}_{\mathrm{j}}, \mathrm{p}_{\mathrm{j}+1}\right\}$, com $\mathrm{p}_{\mathrm{k}+1}=\mathrm{p}_{0}$, pode-se definir para cada $\mathrm{j}=1, \ldots, \mathrm{k}$, um germe em $\mathrm{p}_{\mathrm{j}}$ de uma aplicação de Poincaré $\mathrm{C}^{\mathrm{r}}$ :

$$
\eta_{j}:\left(D, p_{j}\right) \rightarrow\left(D, p_{j+1}\right)
$$

tal que a aplicação de primeiro retorno associada à órbita $\gamma$ é dada por

$$
\eta=\eta_{\mathrm{k}} \circ \eta_{\mathrm{k}-1 \mathrm{o}} \ldots \circ \eta_{0}, \operatorname{com} \eta\left(\mathrm{p}_{0}\right)=\mathrm{p}_{0}
$$

A figura abaixo é um exemplo de poli-trajetória do tipo I.

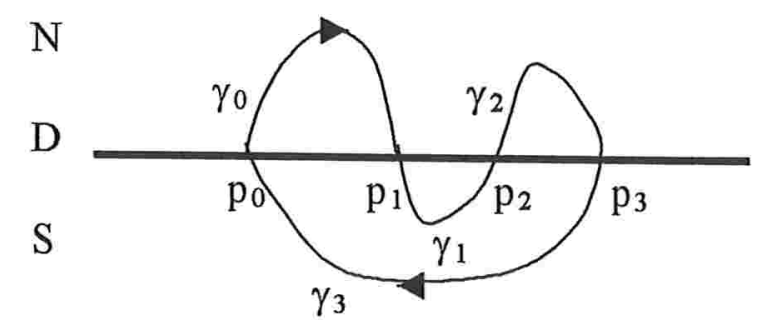

Fig. 26 


\subsection{Definição}

Seja $\gamma$ uma poli-trajetória fechada. Diz-se que $\gamma$ é elementar se

(i) $\quad \gamma$ é do tipo I e $\eta^{\prime}(\mathrm{p}) \neq 1$, para algum $\mathrm{p} \in \gamma$; ou

(ii) $\gamma$ é do tipo II; ou

(iii) $\gamma$ é do tipo III e todos os arcos de trajetória de $\mathrm{F}_{Z}$ são de deslizamento ou todos os arcos são de escape.

Será provado no Capítulo 2 que a regularização das poli-trajetórias fechadas elementares gera órbitas periódicas hiperbólicas.

\section{Regularização de Campos Vetoriais Descontínuos}

Nesta seção é apresentado o método da regularização de um campo vetorial descontínuo, através do qual o mesmo é aproximado por uma família de campos vetoriais contínuos. Este método consiste na utilização de uma função de transição $\varphi$, definida a seguir, com a qual se faz um promédio entre os campos $\mathrm{X}$ e $\mathrm{Y}$ numa vizinhança da curva $\mathrm{D}$. O tamanho desta vizinhança é controlado pelo parâmetro $\varepsilon$, e, para cada valor de $\varepsilon$ fixo, se terá um elemento da família de campos vetoriais regularizados.

\subsection{Definição}

Uma função $\varphi: \mathfrak{R} \rightarrow \mathfrak{R}, \varphi \in \mathrm{C}^{\infty}$, é uma função de transição se

$$
\begin{aligned}
& \varphi(t)=\left\{\begin{array}{l}
0, t \in(-\infty,-1] \\
1, t \in[1, \infty)
\end{array}\right. \\
& \varphi^{\prime}(t)>0 \text { para } t \in(-1,1) .
\end{aligned}
$$

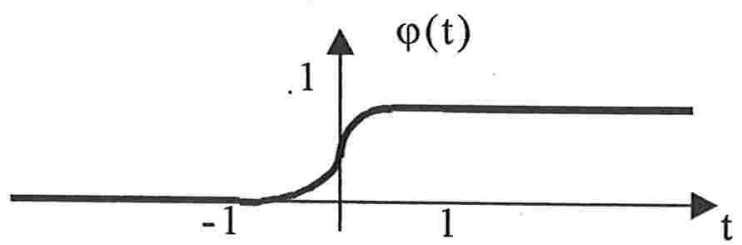

Fig. 27

A figura 27 é exemplo de gráfico de uma função de transição $\varphi$.

\subsection{Definições}

Sejam $\varphi$ uma função de transição e $Z=(X, Y) \in \Omega^{r}(M, f)$ um campo vetorial descontínuo. Uma $\varphi_{\varepsilon}$-regularização de $\mathrm{Z}$ é uma família indexada pelo parâmetro $\varepsilon$, para $\varepsilon>0$, de campos vetoriais $Z_{\varepsilon}$ em $\mathcal{K}^{\mathrm{r}}(\mathrm{M})$ dada por:

$$
Z_{\varepsilon}(q)=\left(1-\varphi_{\varepsilon}(f(q))\right) Y(q)+\varphi_{\varepsilon}(f(q)) X(q), \quad \text { onde } \varphi_{\varepsilon}(t)=\varphi(t / \varepsilon) \text {. }
$$


Nota-se que a função $\varphi$ é estritamente crescente no intervalo $[-1,1]$ e a função $\varphi_{\varepsilon}$ no intervalo $[-\varepsilon, \varepsilon]$.

Para cada valor fixo do parâmetro $\varepsilon>0$, o campo vetorial $Z_{\varepsilon}$ denomina-se campo vetorial regularizado.

Denomina-se faixa de regularização a faixa em torno da curva $\mathrm{D}$ dada por $\left(\varphi_{\varepsilon} \circ f\right)^{-1}(0,1)$, que é a faixa onde o campo $Z_{\varepsilon}$ é definido por um promédio entre o campo X e o campo Y. Ver figura 28.

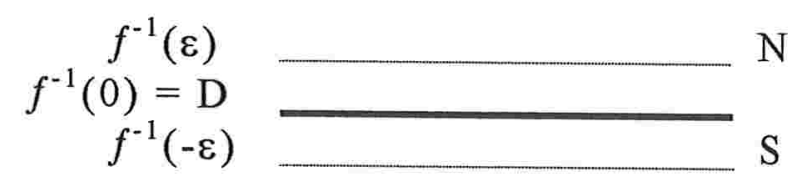

Fig. 28

\subsection{Observação}

Os resultados obtidos neste trabalho não dependem das particulares funções de transição utilizadas, apenas das propriedades comuns às mesmas.

\subsection{Observação}

Utilizando-se o sistema de coordenadas definido na Notação 2.4 , temse $p=(0,0), X \beta(p)=a(0), X f(p)=b(0), Y \beta(p)=c(0)$ e $Y f(p)=d(0)$.

Neste capítulo foram dadas as definições iniciais que serão necessárias na obtenção dos resultados pretendidos. 


\section{Capítulo 2 \\ Campos Vetoriais Descontínuos no Plano}

Neste capítulo são determinadas condições para que a regularização de um campo vetorial descontínuo forneça campos vetoriais de AndronovPontrjaguin-Peixoto (A-P-P). O conjunto obtido é um conjunto genérico, como será demonstrado.

No primeiro parágrafo são estudados o efeito da regularização sobre os pontos regulares, pontos singulares e poli-trajetórias fechadas. Merece destaque a Proposição 1.7, em cuja demonstração é estabelecida, para o caso descontínuo, a fórmula equivalente à da derivada da aplicação de Poincaré do caso contínuo. No segundo parágrafo são estudados os pontos pertencentes à intersecção da curva $\mathrm{D}$ com $\partial \mathrm{M}$. O objetivo é estabelecer as condições para que, durante o processo da regularização, surjam no máximo tangências quadráticas do campo regularizado com $\partial \mathrm{M}$. No terceiro parágrafo é finalmente definido o conjunto de campos vetoriais descontínuos cuja regularização é de A-P-P. É feita a prova deste teorema e também de um teorema de genericidade.

\section{Regularização de Pontos Singulares e Poli-Trajetórias Fechadas}

Nesta seção serão obtidos os principais resultados sobre a regularização em torno de pontos regulares, pontos singulares e politrajetórias fechadas.

A Proposição 1.1 e o Corolário 1.2 tratam dos pontos D-regulares, em cuja vizinhança não surgem pontos singulares durante o processo de regularização. A Proposição 1.1 já havia sido estabelecida por Sotomayor e Teixeira $[\mathrm{S}-\mathrm{T}]$.

\subsection{Proposição}

Seja $p \in D$ um ponto D-regular de $Z=(X, Y) \in \Omega^{r}(M, f), r \geq 1$. Então, dada uma função de transição $\varphi$, existem uma vizinhança $\vartheta$ de $p$ em $M$ e $\varepsilon_{0}>0$ tais que para $0<\varepsilon<\varepsilon_{0}$, o campo vetorial regularizado $Z_{\varepsilon}$ não tem pontos críticos em $\vartheta$. 


\section{demonstração}

Seja $p \in D$ um ponto D-regular de $Z=(X, Y)$. Pela definição, p pode ser um ponto de costura ou um ponto de escape ou deslizamento que não é uma singularidade de $\mathrm{F}_{Z}$.

Usando-se a Notação 2.4 do Capítulo 1, tem-se, em torno de uma vizinhança de $\mathrm{p}$, coordenadas locais tais que $\mathrm{D}=\{\mathrm{y}=0\}, f(\mathrm{x}, \mathrm{y})=\mathrm{y}$, $\mathrm{p}=(0,0), X(x, y)=(a(x, y), b(x, y))$ e $Y(x, y)=(c(x, y), d(x, y))$.

Nesta notação, os dois casos a serem considerados são:

1) Se p é um ponto de costura: $X f(p) Y f(p)=b(0) d(0)>0$;

Pode-se supor, sem perda de generalidade, que

$$
\mathrm{X} f(\mathrm{p})=\mathrm{b}(0)>0 \text { e } \mathrm{Y} f(\mathrm{p})=\mathrm{d}(0)>0 \text {. }
$$

O campo regularizado é dado por

$$
Z_{\varepsilon}(x, y)=\left(\left(1-\varphi_{\varepsilon}(y)\right) c(x, y)+\varphi_{\varepsilon}(y) a(x, y),\left(1-\varphi_{\varepsilon}(y)\right) d(x, y)+\varphi_{\varepsilon}(y) b(x, y)\right) \text {. }
$$

Seja $\vartheta=\{(x, y) \in M \mid b(x, y)>0$ e $d(x, y)>0\}$. É imediato que $\vartheta$ é uma vizinhança de $p$, devido ao fato que $b(0)>0$ e d $(0)>0$ e à continuidade das funções b e d.

Então, $\forall \varepsilon>0$, a segunda coordenada de $Z_{\varepsilon}$ é estritamente positiva nos pontos de $\vartheta$ e portanto $\vartheta$ não contém nenhuma singularidade de $Z_{\varepsilon}$.

As figuras a seguir exemplificam um arco de costura de $Z$ e a sua regularização.

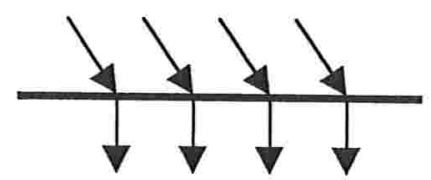

Fig. 29

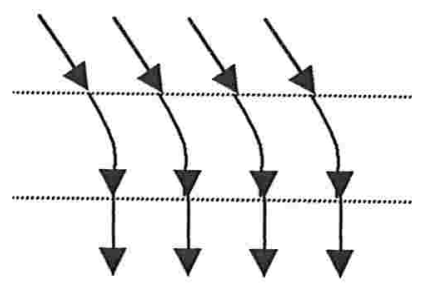

Fig. 30

2) $\mathrm{Se} p$ é um ponto regular de $\mathrm{F}_{Z}$ : $\mathrm{X} f(\mathrm{p}) \mathrm{Y} f(\mathrm{p})=\mathrm{b}(0) \mathrm{d}(0)<0$ e $\operatorname{det}[X, Y](p)=a(0) d(0)-b(0) c(0) \neq 0$.

Pode-se supor, sem perda de generalidade, que $b(0)>0$ e $d(0)<0$.

Seja $\vartheta=\{(x, y) \in M \mid a(x, y) d(x, y)-b(x, y) c(x, y) \neq 0\}$. É imediato que $\vartheta$ é uma vizinhança do ponto $\mathrm{p}$. 
Para que um ponto $(x, y)$ seja uma singularidade do campo regularizado $Z_{\varepsilon}$, deve valer a igualdade

$$
\begin{aligned}
& Z_{\varepsilon}(x, y)=\left(\left(1-\varphi_{\varepsilon}(y)\right) c(x, y)+\varphi_{\varepsilon}(y) a(x, y),\left(1-\varphi_{\varepsilon}(y)\right) d(x, y)+\varphi_{\varepsilon}(y) b(x, y)\right)=(0,0) \\
& \Rightarrow \varphi_{\varepsilon}=c /(c-a), \varphi_{\varepsilon}=d /(d-b) \\
& \Rightarrow d(c-a)=c(d-b) \Rightarrow a d-b c=\operatorname{det}[X, Y]=0 .
\end{aligned}
$$

Logo, tomando-se $(\mathrm{x}, \mathrm{y})$ na vizinhança $\vartheta$, para qualquer $\varepsilon>0$, a equação acima não será satisfeita e $Z_{\varepsilon}$ não possuirá pontos críticos nesta vizinhança.

Observa-se que esta demonstração também é válida se $\mathrm{p} \in \mathrm{D} \cap \partial \mathrm{M}$.

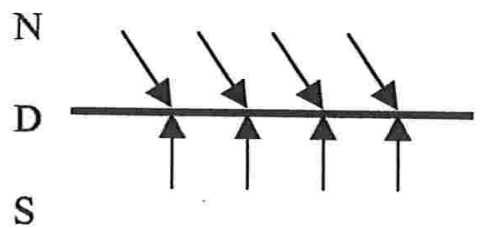

Fig. 31

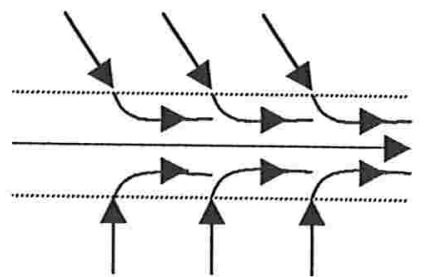

Fig. 32

As figuras acima exemplificam um arco de deslizamento de $Z$ e a sua regularização.

\subsection{Corolário}

Sejam $Z=(X, Y) \in \Omega^{r}(M, f), r \geq 1$, e $T \subset D$ um conjunto compacto de pontos D-regulares. Então, dada uma função de transição $\varphi$, existem $\varepsilon_{0}>0$ e uma vizinhança $\vartheta$ de $T$ em $M$ tais que se $0<\varepsilon<\varepsilon_{0}$, o campo regularizado $Z_{\varepsilon}$ só tem pontos regulares em $\vartheta$.

Os dois próximos lemas mostram como se pode construir um intervalo onde a derivada da função $\varphi_{\varepsilon}$ é maior que uma constante prédeterminada. Estes resultados serão utilizados no próximo parágrafo, na demonstração da ordem da tangência do campo regularizado com $\partial \mathrm{M}$. $\mathrm{O}$ motivo destes lemas estarem aqui posicionados é que uma idéia contida na demonstração destes lemas será utilizada na Proposição 1.5. 


\subsection{Lema}

Seja $h(y)=c(0, y) /(c(0, y)-a(0, y))$. Se $a(0) \cdot c(0)<0$, então existem $\varepsilon_{1}>0$ e constantes $0<h_{0}<h_{1}<1$ tais que $0<h_{0} \leq h(y) \leq h_{1}<1$, para
$|y|<\varepsilon_{1}$.

\section{demonstração}

Da hipótese $\mathrm{a}(0) \mathrm{c}(0)<0$, tem-se que existe $\varepsilon_{1}>0$ tal que a $(0, \mathrm{y})$ e $\mathrm{c}(0, \mathrm{y})$ não se anulam e têm sinais contrários para $|\mathrm{y}|<\varepsilon_{1}$. Pode-se supor, sem perda de generalidade, que $\mathrm{a}(0, \mathrm{y})>0$ e $\mathrm{c}(0, \mathrm{y})<0$.

Então,

$$
\begin{gathered}
c(0, y)-a(0, y)<c(0, y)<0 \\
\Rightarrow 0<\mathrm{h}(y)=c(0, y) /(c(0, y)-a(0, y))<1 .
\end{gathered}
$$

Como h é uma função contínua no fechado $\left[-\varepsilon_{1}, \varepsilon_{1}\right]$, as constantes $\mathrm{h}_{0}=\min \left\{\mathrm{h}(\mathrm{y}): \mathrm{y} \in\left[-\varepsilon_{1}, \varepsilon_{1}\right]\right\}$ e $\mathrm{h}_{1}=\max \left\{\mathrm{h}(\mathrm{y}): \mathrm{y} \in\left[-\varepsilon_{1}, \varepsilon_{1}\right]\right\}$ são tais que $0<\mathrm{h}_{0}<\mathrm{h}_{1}<1$.

Observa-se que se $\mathrm{a}(0, \mathrm{y})<0$ e $\mathrm{c}(0, \mathrm{y})>0$, o raciocínio é análogo.

\subsection{Lema}

Seja $Z=(X, Y) \in \Omega^{r}(M, f)$, onde $X=(a, b)$ e $Y=(c, d)$ são tais que $a(0) . c(0)<0$. Dados $K>0$ e uma função de transição $\varphi$, existe $\varepsilon_{2}>0$ tal que se $0<\varepsilon<\varepsilon_{2}$ e $y \in \Re$ satisfaz $\varphi_{\varepsilon}(y) \in\left[h_{0}, h_{1}\right]$, onde $h_{0}$ e $h_{1}$ são as constantes obtidas no Lema 1.3, então $\varphi_{\varepsilon}^{\prime}(y)>K$.

demonstração

Sejam $\varepsilon_{1}, \mathrm{~h}_{0}$ e $\mathrm{h}_{1}$ dados pelo Lema 1.3.

Sejam $0<\varepsilon^{\prime}<\varepsilon^{\prime \prime}<\varepsilon_{1}$ e $y^{\prime}$ e $y^{\prime \prime}$ tais que $0<\varphi_{\varepsilon^{\prime}}\left(y^{\prime}\right)=\varphi_{\varepsilon^{\prime}}\left(y^{\prime \prime}\right)<1$. Afirma-se que $\varphi^{\prime}{ }_{\varepsilon^{\prime}}\left(\mathrm{y}^{\prime}\right)>\varphi^{\prime}{ }_{\varepsilon^{\prime \prime}}\left(\mathrm{y}^{\prime \prime}\right)$.

De fato, $\varphi_{\varepsilon}(\mathrm{y})=\varphi(\mathrm{y} / \varepsilon)$, e $\varphi^{\prime}{ }_{\varepsilon}(\mathrm{y})=(1 / \varepsilon) \varphi^{\prime}(\mathrm{y} / \varepsilon)$.

$\operatorname{Logo}, \varphi_{\mathrm{s}}\left(\mathrm{y}^{\prime}\right)=\varphi\left(\mathrm{y}^{\prime} / \varepsilon^{\prime}\right)=\varphi\left(\mathrm{y}^{\prime \prime} / \varepsilon^{\prime \prime}\right)=\varphi_{\varepsilon^{\prime \prime}}\left(\mathrm{y}^{\prime \prime}\right)$.

Como esta igualdade ocorre no trecho em que a $\varphi$ é injetora, tem-se $\mathrm{y}^{\prime} / \varepsilon^{\prime}=\mathrm{y}^{\prime \prime} / \varepsilon^{\prime \prime}$.

Logo, $\varphi^{\prime}\left(\mathrm{y}^{\prime} / \varepsilon^{\prime}\right)=\varphi^{\prime}\left(\mathrm{y}^{\prime \prime} / \varepsilon^{\prime \prime}\right)$, e como $\varepsilon^{\prime}<\varepsilon^{\prime \prime}$, 


$$
\varphi_{\varepsilon^{\prime}}^{\prime}\left(y^{\prime}\right)=\left(1 / \varepsilon^{\prime}\right) \varphi^{\prime}\left(y^{\prime} / \varepsilon^{\prime}\right)>\left(1 / \varepsilon^{\prime \prime}\right) \varphi^{\prime}\left(y^{\prime \prime} / \varepsilon^{\prime \prime}\right)=\varphi_{\varepsilon^{\prime \prime}}^{\prime \prime}\left(y^{\prime \prime}\right)
$$

Ou seja, diminuindo-se o valor de $\varepsilon$ o valor de $\varphi^{\prime}$ tende a aumentar.

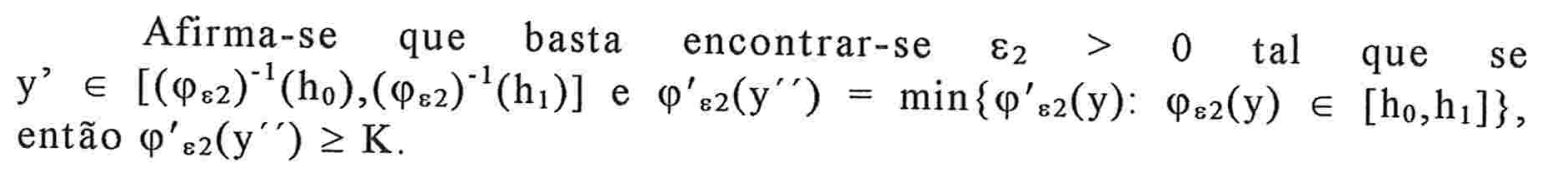

De fato, basta porque se $0<\varepsilon<\varepsilon_{2}$ e $\mathrm{y}^{\prime}$ é tal que $\varphi_{\varepsilon}\left(\mathrm{y}^{\prime}\right)=\varphi_{\varepsilon 2}\left(\mathrm{y}^{\prime \prime}\right)$, então $\varphi_{\varepsilon}^{\prime}\left(\mathrm{y}^{\prime}\right)=\min \left\{\varphi^{\prime}{ }_{\varepsilon}(\mathrm{y}): \varphi_{\varepsilon}(\mathrm{y}) \in\left[\mathrm{h}_{0}, \mathrm{~h}_{1}\right]\right\}$, e $\varphi^{\prime}{ }_{\varepsilon}\left(y^{\prime}\right)>\varphi_{\varepsilon 2}^{\prime}\left(y^{\prime \prime}\right)$, pelo que foi demonstrado.

Sejam $\varepsilon>0$ e $y^{\prime}$ tal que $\varphi^{\prime}{ }_{\varepsilon}\left(y^{\prime}\right)=\min \left\{\varphi^{\prime}{ }_{\varepsilon}(y): \varphi_{\varepsilon}(y) \in\left[h_{0}, h_{1}\right]\right\}$ e seja $\varepsilon_{2}=\varepsilon \varphi^{\prime}{ }_{\mathrm{\varepsilon}}\left(\mathrm{y}^{\prime}\right) / \mathrm{K}$.

Então se $y^{\prime \prime}$ é o ponto no qual $\varphi_{\varepsilon 2}^{\prime}$ é mínimo, isto é, $\varphi_{\varepsilon}\left(y^{\prime}\right)=\varphi_{\varepsilon 2}\left(y^{\prime \prime}\right)$, tem-se

$$
\begin{aligned}
\varphi^{\prime}{ }_{\varepsilon 2}\left(y^{\prime \prime}\right) & \left.=\left(1 / \varepsilon_{2}\right) \varphi^{\prime}\left(y^{\prime \prime} / \varepsilon_{2}\right)=\left(1 / \varepsilon_{2}\right) \varphi^{\prime}\left(y^{\prime} / \varepsilon\right)=K / \varepsilon\right)\left(1 / \varphi^{\prime}{ }_{\varepsilon}\left(y^{\prime}\right)\right) \varphi^{\prime}\left(y^{\prime} / \varepsilon\right)= \\
= & K\left(1 / \varphi_{\varepsilon}^{\prime}\left(y^{\prime}\right)\right) \varphi^{\prime}{ }_{\varepsilon}\left(y^{\prime}\right)=K .
\end{aligned}
$$

Com isto, o Lema está demonstrado.

A seguinte Proposição trata sobre a regularização de um ponto crítico hiperbólico de $\mathrm{F}_{Z}$. A mesma foi estabelecida por Sotomayor e Teixeira [S-T]. Neste trabalho foram fornecidos os detalhes da demonstração desta Proposição.

\subsection{Proposição}

Dado $Z=(X, Y) \in \Omega^{r}(M, f), \quad r \geq 1$, seja $p$ um ponto crítico hiperbólico de $F_{Z}$. Então, dada uma função de transição $\varphi$, existem $\varepsilon_{0}>0$ $e$ uma vizinhança $\vartheta$ de $p$ em $M$ tal que para cada $0<\varepsilon<\varepsilon_{0}$, o campo regularizado $Z_{\varepsilon}$ tem um único ponto crítico $p_{\varepsilon}$ em $\vartheta$, que é hiperbólico e do tipo sela ou nó, conforme $p$ ofor para $Z$.

demonstração

Por hipótese, tem-se

$$
\mathrm{X} f(\mathrm{p}) . \mathrm{Y} f(\mathrm{p})<0, \operatorname{det}[\mathrm{X}, \mathrm{Y}](\mathrm{p})=0 \mathrm{ed}(\operatorname{det}[\mathrm{X}, \mathrm{Y}](\mathrm{p})) \neq 0 .
$$


Será considerado o caso em que $\mathrm{X} f(\mathrm{p})<0$ e $\mathrm{Y} f(\mathrm{p})>0$, isto é, o ponto $\mathrm{p}$ pertence a um arco de deslizamento. $\mathrm{O}$ caso em que o ponto $\mathrm{p}$ pertence a um arco de escape é análogo.

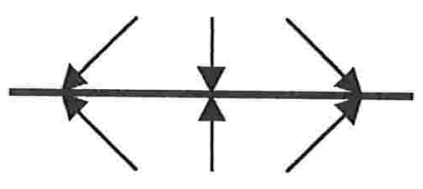

Fig. 33

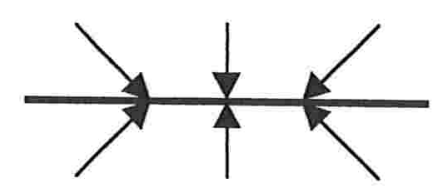

Fig. 35

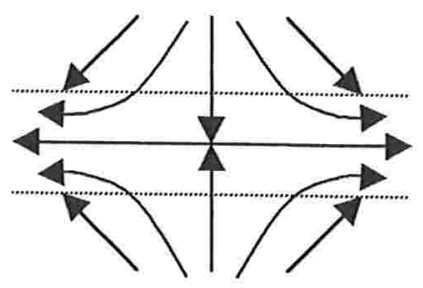

Fig. 34

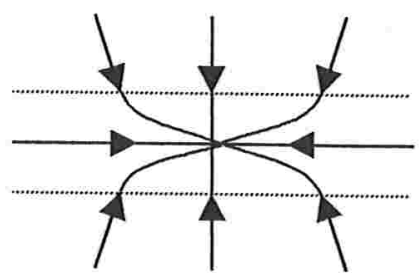

Fig. 36

As figuras 33 e 34 exemplificam uma sela de $\mathrm{F}_{Z}$ e a sua regularização e as figuras 35 e 36 exemplificam um nó atrator de $F_{Z}$ e a sua regularização.

Pela Notação 2.4 do Capítulo 1 , ao redor de $p=(0,0)$ tem-se $f(\mathrm{x}, \mathrm{y})=\mathrm{y}, \mathrm{X}(\mathrm{x}, \mathrm{y})=(\mathrm{a}(\mathrm{x}, \mathrm{y}), \mathrm{b}(\mathrm{x}, \mathrm{y}))$ e $\mathrm{Y}(\mathrm{x}, \mathrm{y})=(\mathrm{c}(\mathrm{x}, \mathrm{y}), \mathrm{d}(\mathrm{x}, \mathrm{y}))$.

Nesta notação, tem-se

$$
\begin{aligned}
& \mathrm{X} f(\mathrm{p})=\mathrm{b}(0)<0, Y f(\mathrm{p})=\mathrm{d}(0)>0, \\
& \operatorname{det}[\mathrm{X}, \mathrm{Y}](\mathrm{x}, \mathrm{y})=\mathrm{a}(\mathrm{x}, \mathrm{y}) \mathrm{d}(\mathrm{x}, \mathrm{y})-\mathrm{b}(\mathrm{x}, \mathrm{y}) \mathrm{c}(\mathrm{x}, \mathrm{y}), \\
& \operatorname{det}[\mathrm{X}, \mathrm{Y}](\mathrm{p})=\mathrm{a}(0) \mathrm{d}(0)-\mathrm{b}(0) \mathrm{c}(0)=0 \mathrm{e} \\
& \mathrm{d}\left(\left.\operatorname{det}[\mathrm{X}, \mathrm{Y}]\right|_{\mathrm{D}}\right)(\mathrm{p})=(\partial / \partial \mathrm{x})(\operatorname{det}[\mathrm{X}, \mathrm{Y}](\mathrm{p}))= \\
& =\mathrm{a}_{\mathrm{x}}(0) \mathrm{d}(0)+\mathrm{a}(0) \mathrm{d}_{\mathrm{x}}(0)-\mathrm{b}_{\mathrm{x}}(0) \mathrm{c}(0)-\mathrm{b}(0) \mathrm{c}_{\mathrm{x}}(0) \neq 0 .
\end{aligned}
$$

O campo vetorial regularizado toma a forma

$$
Z_{\varepsilon}=\left(Z_{\varepsilon}^{1}, Z^{2}{ }_{\varepsilon}\right)=\left(\left(1-\varphi_{\varepsilon}\right) c+\varphi_{\varepsilon} a,\left(1-\varphi_{\varepsilon}\right) d+\varphi_{\varepsilon} b\right) .
$$

Os pontos críticos de $Z_{\varepsilon}$ são as soluções de 


$$
\left\{\begin{array}{ll}
\left(1-\varphi_{\varepsilon}\right) c+\varphi_{\varepsilon} a & =0 \\
\left(1-\varphi_{\varepsilon}\right) d+\varphi_{\varepsilon} b & =0
\end{array} \quad \Rightarrow \quad \varphi_{\varepsilon}(y)=\frac{c}{c-a}=\frac{d}{d-b}\right.
$$

Será mostrado que existe uma curva na qual vale $\frac{c}{c-a}=\frac{d}{d-b}$, e que, para $\varepsilon$ pequeno o suficiente, esta curva só cruza uma vez o gráfico de $\varphi_{\varepsilon}$.

Da igualdade $\frac{\mathrm{c}}{\mathrm{c}-\mathrm{a}}=\frac{\mathrm{d}}{\mathrm{d}-\mathrm{b}}$ segue $\mathrm{ad}-\mathrm{bc}=0$, ou seja, qualquer ponto crítico $p_{\varepsilon}$ de $Z_{\varepsilon}$ deve satisfazer $\operatorname{det}[X, Y]\left(p_{\varepsilon}\right)=0$.

Como $(\partial / \partial \mathrm{x})(\operatorname{det}[\mathrm{X}, \mathrm{Y}](\mathrm{p})) \neq 0$, pelo Teorema das Funções Implícitas, existem um aberto IxJ, com $\mathrm{p}=(0,0) \in \mathrm{IxJ}$ e uma função diferenciável $\alpha: J \rightarrow I$ tal que $\operatorname{det}[X, Y](\alpha(y), y)=0$.

Nos pontos da curva $(\alpha(y), y)$ vale a igualdade $\frac{c}{c-a}=\frac{d}{d-b}$.

Deve-se mostrar que existe apenas um ponto $p_{\varepsilon}$ nesta curva no qual vale também (1).

De fato, como $\mathrm{b}(0)<0$ e $\mathrm{d}(0)>0$, pode-se tomar $\varepsilon_{0}>0$ de modo que estas duas funções não mudem de sinal se $|\mathrm{x}| \leq \varepsilon_{0}$ e $|\mathrm{y}| \leq \varepsilon_{0}$, e tenha-se $J \subset\left[-\varepsilon_{0}, \varepsilon_{0}\right]$.

Define-se $\vartheta=\left\{(\mathrm{x}, \mathrm{y}) \in \mathrm{M}|| \mathrm{x} \mid \leq \varepsilon_{0}\right.$ e $\left.|\mathrm{y}| \leq \varepsilon_{0}\right\}$

Logo, para $(x, y) \in \vartheta$ tem-se $\frac{d(x, y)}{d(x, y)-b(x, y)} \in(0,1)$.

Seja $g(y)=\frac{d(\alpha(y), y)}{(d(\alpha(y), y)-b(\alpha(y), y))}$

Sejam $\mathrm{k}=\frac{\mathrm{d}(0)}{\mathrm{d}(0)-\mathrm{b}(0)}$ e $\mathrm{m}=\min \{\mathrm{k}, 1-\mathrm{k}\}$. Como $\mathrm{k} \in(0,1)$, pode-se diminuir $\varepsilon_{0}$ de modo que $\frac{d(x, y)}{d(x, y)-b(x, y)} \in(k-m / 2, k+m / 2)$, se $(x, y) \in \vartheta$.

Como $\varphi_{\varepsilon}\left(-\varepsilon_{0}\right)=0, \varphi_{\varepsilon}\left(\varepsilon_{0}\right)=1$, e as funções $\varphi_{\varepsilon}(y)$ e $g(y)$ são contínuas, existe pelo menos um ponto de cruzamento dos dois gráficos.

Resta provar que este ponto de cruzamento é único. 
De fato, se não for único, como $\varphi_{\varepsilon 0}(y)$ é uma função estritamente crescente apenas no intervalo $\left[-\varepsilon_{0}, \varepsilon_{0}\right]$, basta diminuir o valor de $\varepsilon_{0}$ até que o cruzamento só ocorra uma vez, como ilustra a figura 37 . O mesmo é válido para $0<\varepsilon<\varepsilon_{0}$.

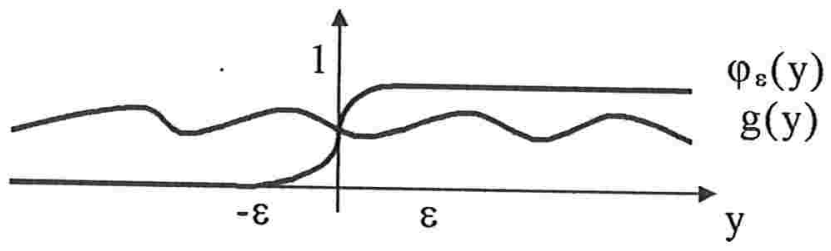

Fig. 37

Seja $y_{\varepsilon}$ este único ponto de cruzamento dos dois gráficos, para $0<\varepsilon<\varepsilon_{0}$. Então, $p_{\varepsilon}=\left(\alpha\left(y_{\varepsilon}\right), y_{\varepsilon}\right)$ é a única singularidade de $Z_{\varepsilon}$ em $\vartheta$.

Afirma-se que $p_{\varepsilon}$ é uma singularidade hiperbólica, e é uma sela se $p$ for sela para $F_{Z}$ ou um nó se $p$ for nó para $F_{Z}$.

De fato, a matriz $\mathrm{DZ}_{\varepsilon}$, calculada em $\mathrm{p}_{\varepsilon}$ é dada por

$$
\mathrm{DZ}_{\varepsilon}=\left[\begin{array}{cc}
\left(1-\varphi_{\varepsilon}\right) \mathrm{c}_{\mathrm{x}}+\varphi_{\varepsilon} \mathrm{a}_{\mathrm{x}} & -\left(\varphi_{\varepsilon}\right)^{\prime} \mathrm{c}+\left(1-\varphi_{\varepsilon}\right) \mathrm{c}_{\mathrm{y}}+\left(\varphi_{\varepsilon}\right)^{\prime} \mathrm{a}+\varphi_{\varepsilon} \mathrm{a}_{\mathrm{y}} \\
\left(1-\varphi_{\varepsilon}\right) \mathrm{d}_{\mathrm{x}}+\varphi_{\varepsilon} \mathrm{b}_{\mathrm{x}} & -\left(\varphi_{\varepsilon}\right)^{\prime} \mathrm{d}+\left(1-\varphi_{\varepsilon}\right) \mathrm{d}_{\mathrm{y}}+\left(\varphi_{\varepsilon}\right)^{\prime} \mathrm{b}+\varphi_{\varepsilon} \mathrm{b}_{\mathrm{y}}
\end{array}\right]
$$

E o polinômio característico é dado por

$\left|\mathrm{D} Z_{\varepsilon}-\lambda \mathrm{I}\right|=\left(\left(1-\varphi_{\varepsilon}\right) \mathrm{c}_{\mathrm{x}}+\varphi_{\varepsilon} \mathrm{a}_{\mathrm{x}}-\lambda\right)\left(-\left(\varphi_{\varepsilon}\right)^{\prime} \mathrm{d}+\left(1-\varphi_{\varepsilon}\right) \mathrm{d}_{\mathrm{y}}+\left(\varphi_{\varepsilon}\right)^{\prime} \mathrm{b}+\varphi_{\varepsilon} \mathrm{b}_{\mathrm{y}}-\lambda\right)$ $-\left(\left(1-\varphi_{\varepsilon}\right) d_{x}+\varphi_{\varepsilon} b_{x}\right)\left(-\left(\varphi_{\varepsilon}\right)^{\prime} c+\left(1-\varphi_{\varepsilon}\right) c_{y}+\left(\varphi_{\varepsilon}\right)^{\prime} a+\varphi_{\varepsilon} a_{y}\right)=0$.

$\Rightarrow \quad \lambda^{2}+\lambda\left(-\left(1-\varphi_{\varepsilon}\right) c_{x}-\varphi_{\varepsilon} a_{x}+\left(\varphi_{\varepsilon}\right)^{\prime} d-\left(1-\varphi_{\varepsilon}\right) d_{y}-\left(\varphi_{\varepsilon}\right)^{\prime} b-\varphi_{\varepsilon} b_{y}\right)-$ $\left(\varphi_{\varepsilon}\right)^{\prime}\left(1-\varphi_{\varepsilon}\right) c_{x} d+\left(1-\varphi_{\varepsilon}\right)^{2} c_{x} d_{y}+\left(\varphi_{\varepsilon}\right)^{\prime}\left(1-\varphi_{\varepsilon}\right) b c_{x}+\varphi_{\varepsilon}\left(1-\varphi_{\varepsilon}\right) b_{y} c_{x}-\left(\varphi_{\varepsilon}\right)^{\prime} \varphi_{\varepsilon} a_{x} d+$ $\varphi_{\varepsilon}\left(1-\varphi_{\varepsilon}\right) \mathrm{a}_{\mathrm{x}} \mathrm{d}_{\mathrm{y}}+\left(\varphi_{\varepsilon}\right)^{\prime} \varphi_{\varepsilon} \mathrm{a}_{\mathrm{x}} \mathrm{b}+\left(\varphi_{\varepsilon}\right)^{2} \mathrm{a}_{\mathrm{x}} \mathrm{b}_{\mathrm{y}}+\left(\varphi_{\varepsilon}\right)^{\prime}\left(1-\varphi_{\varepsilon}\right) \mathrm{cd}_{\mathrm{x}}-\left(1-\varphi_{\varepsilon}\right)^{2} \mathrm{c}_{\mathrm{y}} \mathrm{d}_{\mathrm{x}}-$ $\left(\varphi_{\varepsilon}\right)^{\prime}\left(1-\varphi_{\varepsilon}\right) a_{x}-\varphi_{\varepsilon}\left(1-\varphi_{\varepsilon}\right) a_{y} d_{x}+\left(\varphi_{\varepsilon}\right)^{\prime} \varphi_{\varepsilon} b_{x} c-\varphi_{\varepsilon}\left(1-\varphi_{\varepsilon}\right) b_{x} c_{y}-\left(\varphi_{\varepsilon}\right)^{\prime} \varphi_{\varepsilon} a b_{x}-$ $\left(\varphi_{\varepsilon}\right)^{2} a_{y} b_{x}=0$.

Observa-se que para uma singularidade com auto-valores dados por $\lambda=1 / 2\left(-b_{\lambda} \pm \sqrt{ } \Delta_{\lambda}\right)$ ser uma sela hiperbólica deve-se ter

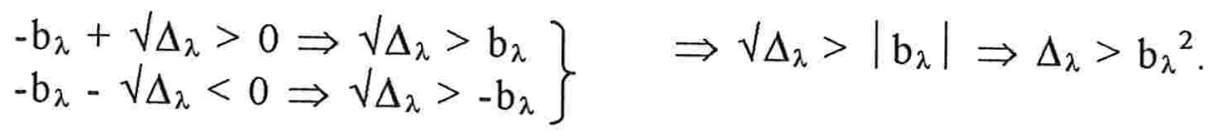

E para esta singularidade ser um nó hiperbólico deve-se ter

$$
\left(-\mathrm{b}_{\lambda}+\sqrt{\Delta_{\lambda}}\right)\left(-\mathrm{b}_{\lambda}-\sqrt{\Delta_{\lambda}}\right)>0 \Rightarrow \mathrm{b}_{\lambda}{ }^{2}-\Delta_{\lambda}>0 \Rightarrow 0 \leq \Delta_{\lambda}<\mathrm{b}_{\lambda}{ }^{2}
$$


Tem-se

$\Delta_{\lambda}-b_{\lambda}^{2}=b_{\lambda}^{2}-4 a_{\lambda} c_{\lambda}-b_{\lambda}^{2}=-4 a_{\lambda} c_{\lambda}$.

Como $\mathrm{a}_{\lambda}=1$, basta analisar-se o sinal de $c_{\lambda}$.

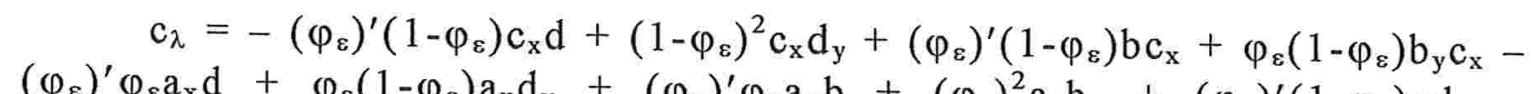
$\left(\varphi_{\varepsilon}\right)^{\prime} \varphi_{\varepsilon} a_{x} d+\varphi_{\varepsilon}\left(1-\varphi_{\varepsilon}\right) a_{x} d_{y}+\left(\varphi_{\varepsilon}\right)^{\prime} \varphi_{\varepsilon} a_{x} b+\left(\varphi_{\varepsilon}\right)^{2} a_{x} b_{y}+\left(\varphi_{\varepsilon}\right)^{\prime}\left(1-\varphi_{\varepsilon}\right) c d_{x}-$ $\left(1-\varphi_{\varepsilon}\right)^{2} c_{y} d_{x}-\left(\varphi_{\varepsilon}\right)^{\prime}\left(1-\varphi_{\varepsilon}\right) a d_{x}-\varphi_{\varepsilon}\left(1-\varphi_{\varepsilon}\right) a_{y} d_{x}+\left(\varphi_{\varepsilon}\right)^{\prime} \varphi_{\varepsilon} b_{x} c-\varphi_{\varepsilon}\left(1-\varphi_{\varepsilon}\right) b_{x} c_{y}-$ $\left(\varphi_{\varepsilon}\right)^{\prime} \varphi_{\varepsilon} a b_{x}-\left(\varphi_{\varepsilon}\right)^{2} a_{y} b_{x}=$

$=\left(\varphi_{\varepsilon}\right)^{\prime}\left(1-\varphi_{\varepsilon}\right)\left(-c_{x} d+b c_{x}+c d_{x}-a d_{x}\right)+\left(1-\varphi_{\varepsilon}\right)^{2}\left(c_{x} d_{y}-c_{y} d_{x}\right)+$ $\varphi_{\varepsilon}\left(1-\varphi_{\varepsilon}\right)\left(b_{y} c_{x}+a_{x} d_{y}-a_{y} d_{x}-b_{x} c_{y}\right)+\left(\varphi_{\varepsilon}\right)^{\prime} \varphi_{\varepsilon}\left(-a_{x} d+a_{x} b+b_{x} c-a b_{x}\right)+$ $\left(\varphi_{\varepsilon}\right)^{2}\left(a_{x} b_{y}-a_{y} b_{x}\right)=$

$=\left(\varphi_{\varepsilon}\right)^{\prime}\left(1-\varphi_{\varepsilon}\right)\left(c_{x}(b-d)+d_{x}(c-a)\right)+\left(1-\varphi_{\varepsilon}\right)^{2}\left(c_{x} d_{y}-c_{y} d_{x}\right)+$ $\varphi_{\varepsilon}\left(1-\varphi_{\varepsilon}\right)\left(b_{y} c_{x}+a_{x} d_{y}-a_{y} d_{x}-b_{x} c_{y}\right)+\left(\varphi_{\varepsilon}\right)^{\prime} \varphi_{\varepsilon}\left(a_{x}(b-d)+b_{x}(c-a)\right)+$ $\left(\varphi_{\varepsilon}\right)^{2}\left(a_{x} b_{y}-a_{y} b_{x}\right)=$

De (1) tem-se

$$
\begin{aligned}
& c_{\lambda}=\left(\varphi_{\varepsilon}\right)^{\prime}\left(1-\varphi_{\varepsilon}\right)\left(-d_{x} / \varphi_{\varepsilon}+c_{x} / \varphi_{\varepsilon}\right)+\left(1-\varphi_{\varepsilon}\right)^{2}\left(c_{x} d_{y}-c_{y} d_{x}\right)+ \\
& \varphi_{\varepsilon}\left(1-\varphi_{\varepsilon}\right)\left(b_{y} c_{x}+a_{x} d_{y}-a_{y} d_{x}-b_{x} c_{y}\right)+\left(\varphi_{\varepsilon}\right)^{\prime} \varphi_{\varepsilon}\left(-a_{x} d / \varphi_{\varepsilon}+b_{x} c / \varphi_{\varepsilon}\right)+ \\
& \left(\varphi_{\varepsilon}\right)^{2}\left(a_{x} b_{y}-a_{y} b_{x}\right)= \\
& =\left(\varphi_{\varepsilon}\right)^{\prime}\left(-d c_{x} / \varphi_{\varepsilon}+c d_{x} / \varphi_{\varepsilon}\right)+\left(\varphi_{\varepsilon}\right)^{\prime}\left(d c_{x}-c d_{x}\right)+\left(1-2 \varphi_{\varepsilon}+\left(\varphi_{\varepsilon}\right)^{2}\right) \\
& \left(c_{x} d_{y}-c_{y} d_{x}\right)+\left(\varphi_{\varepsilon}-\left(\varphi_{\varepsilon}\right)^{2}\right)\left(b_{y} c_{x}+a_{x} d_{y}-a_{y} d_{x}-b_{x} c_{y}\right)+\left(\varphi_{\varepsilon}\right)^{\prime}\left(-a_{x} d+b_{x} c\right)+ \\
& \left(\varphi_{\varepsilon}\right)^{2}\left(a_{x} b_{y}-a_{y} b_{x}\right)= \\
& =\left(\varphi_{\varepsilon}\right)^{\prime}\left(-d c_{x} / \varphi_{\varepsilon}+c d_{x} / \varphi_{\varepsilon}\right)+\left(\varphi_{\varepsilon}\right)^{\prime}\left(d c_{x}-c d_{x}-a_{x} d+b_{x} c\right)+\left(c_{x} d_{y}-\right. \\
& \left.c_{y} d_{x}\right)+\varphi_{\varepsilon}\left(-2 c_{x} d_{y}+2 c_{y} d_{x}+b_{y} c_{x}+a_{x} d_{y}-a_{y} d_{x}-b_{x} c_{y}\right)+\left(\varphi_{\varepsilon}\right)^{2}\left(c_{x} d_{y}-c_{y} d_{x}-\right. \\
& \left.b_{y} c_{x}-a_{x} d_{y}+a_{y} d_{x}+b_{x} c_{y}\right)= \\
& \quad=\left(\varphi_{\varepsilon}\right)^{\prime}\left(b c_{x}-d c_{x}+c d_{x}-a d_{x}+d_{x}-c d_{x}-a_{x} d+b_{x} c\right)+\left(c_{x} d_{y}-c_{y} d_{x}\right) \\
& +\varphi_{\varepsilon}\left(-2 c_{x} d_{y}+2 c_{y} d_{x}+b_{y} c_{x}+a_{x} d_{y}-a_{y} d_{x}-b_{x} c_{y}\right)+\left(\varphi_{\varepsilon}\right)^{2}\left(c_{x} d_{y}-c_{y} d_{x}-b_{y} c_{x}-\right. \\
& \left.a_{x} d_{y}+a_{y} d_{x}+b_{x} c_{y}\right)= \\
& =\left(\varphi_{\varepsilon}\right)^{\prime}\left(b c_{x}-a d_{x}-a_{x} d+b_{x} c\right)+L=-\left(\varphi_{\varepsilon}\left(p_{\varepsilon}\right)\right)^{\prime} d\left(d e t[X, Y]\left(p_{\varepsilon}\right)\right)+L\left(p_{\varepsilon}\right) . \\
& \quad O n d e L\left(p_{\varepsilon}\right)=\left(c_{x} d_{y}-c_{y} d_{x}\right)+\varphi_{\varepsilon}\left(-2 c_{x} d_{y}+2 c_{y} d_{x}+b_{y} c_{x}+a_{x} d_{y}-a_{y} d_{x}-\right. \\
& \left.b_{x} c_{y}\right)+\left(\varphi_{\varepsilon}\right)^{2}\left(c_{x} d_{y}-c_{y} d_{x}-b_{y} c_{x}-a_{x} d_{y}+a_{y} d_{x}+b_{x} c_{y}\right) e ́ u m a \text { função limitada. }
\end{aligned}
$$

Portanto, resta provar que $\lim _{\varepsilon \rightarrow 0}\left(\varphi_{\varepsilon}\left(\mathrm{p}_{\varepsilon}\right)\right)^{\prime}=\infty$, para se concluir que o 
sinal de $c_{\lambda}$ é o mesmo de $-d\left(\operatorname{det}[X, Y]\left(p_{\varepsilon}\right)\right)$, ou seja, que $p_{\varepsilon}$ é uma sela hiperbólica de $Z_{\varepsilon}$ se $p$ o for para $F_{Z}$ ou um nó hiperbólico de $Z_{\varepsilon}$ se $p$ o for para $F_{Z}$.

De fato, a afirmação acima decorre de $\varphi_{\varepsilon}\left(p_{\varepsilon}\right) \in(k-m / 2, k+m / 2)$ e $\mathrm{k}$ não depender de $\varepsilon$, usando-se um raciocínio análogo ao da demonstração do Lema 1.4.

A seguinte Proposição trata da regularização em torno de pontos do tipo D-dobra, e mostra que não surgem pontos críticos em vizinhanças das D-dobras, em decorrência da regularização. A mesma foi estabelecida por Sotomayor e Teixeira [S-T].

\subsection{Proposição}

Seja $p$ uma D-dobra de $Z=(X, Y) \in \Omega^{r}(M, f), r \geq 1$. Então, dada uma função de transição $\varphi$, existem uma vizinhança $\vartheta$ de $p$ em $M$ e $\varepsilon_{0}>0$ tal que se $0<\varepsilon<\varepsilon_{0}$, o campo vetorial regularizado $Z_{\varepsilon}$ não possui pontos críticos em $\vartheta$.

demonstração

Pode-se assumir, sem perda de generalidade, que $\mathrm{Xf}(\mathrm{p})=0$, $\mathrm{X}^{2} f(\mathrm{p})>0$ e $\mathrm{Y} f(\mathrm{p})>0$. Os outros casos são análogos.

As coordenadas utilizadas são tais que numa vizinhança de $\mathrm{p}=(0,0)$, tem-se $X(x, y)=(a(x, y), b(x, y)), Y(x, y)=(c(x, y), d(x, y))$ e $f(\mathrm{x}, \mathrm{y})=\mathrm{y}$.

Logo,

$\mathrm{X} f(\mathrm{p})=\mathrm{b}(0)=0$

$X^{2} f(p)=(a(0), b(0)) \cdot\left(b_{x}(0), b_{y}(0)\right)=a(0) b_{x}(0)+b(0) b_{y}(0)=a(0) b_{x}(0)>0$

$\Rightarrow \quad \mathrm{a}(0) \neq 0$

e $\quad \mathrm{Y} f(\mathrm{p})=\mathrm{d}(0)>0$.

O campo regularizado toma a forma

$Z_{\varepsilon}=\left(Z_{\varepsilon}^{1}, Z^{2}{ }_{\varepsilon}\right)=\left(\left(1-\varphi_{\varepsilon}\right) c+\varphi_{\varepsilon} a,\left(1-\varphi_{\varepsilon}\right) d+\varphi_{\varepsilon} b\right)$ 
Para que $Z_{\varepsilon}$ tenha um ponto singular deve-se ter

$$
\begin{aligned}
& \left\{\begin{array}{l}
\left(1-\varphi_{\varepsilon}(y)\right) c(x, y)+a(x, y) \varphi_{\varepsilon}(y)=0 \\
\left(1-\varphi_{\varepsilon}(y)\right) d(x, y)+b(x, y) \varphi_{\varepsilon}(y)=0
\end{array}\right. \\
\Rightarrow \quad & \left\{\begin{array}{l}
\varphi_{\varepsilon}(y)(a(x, y)-c(x, y))=-c(x, y) \\
\varphi_{\varepsilon}(y)(b(x, y)-d(x, y))=-d(x, y)
\end{array}\right. \\
\Rightarrow \quad & \varphi_{\varepsilon}(y)=\frac{c(x, y)}{c(x, y)-a(x, y)}=\frac{d(x, y)}{d(x, y)-b(x, y)} \\
\Rightarrow \quad & a(x, y) d(x, y)-b(x, y) c(x, y)=\operatorname{det}[X, Y](x, y)=0
\end{aligned}
$$

No ponto $p$, tem-se

$$
\operatorname{det}[X, Y](p)=a(0) d(0)-b(0) c(0)=a(0) d(0)-0 . c(0)=a(0) d(0) \neq 0
$$

Sejam $\varepsilon_{0}>0$ tal que $\operatorname{det}[X, Y](x, y) \neq 0$ se $|x| \leq \varepsilon_{0}$ e $|y| \leq \varepsilon_{0}$ e $\vartheta=\left\{(\mathrm{x}, \mathrm{y}) \in \mathrm{M}|| \mathrm{x} \mid \leq \varepsilon_{0}\right.$ e $\left.|\mathrm{y}| \leq \varepsilon_{0}\right\}$. É imediato que $\vartheta$ é uma vizinhança de $p$ e se $0<\varepsilon<\varepsilon_{0}$, então o campo regularizado $Z_{\varepsilon}$ não possui singularidade em $\vartheta$, e com isto conclui-se a demonstração da Proposição.

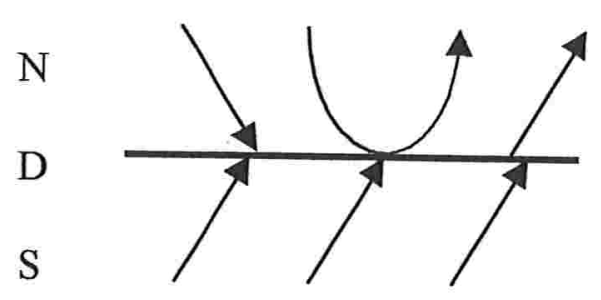

Fig. 38

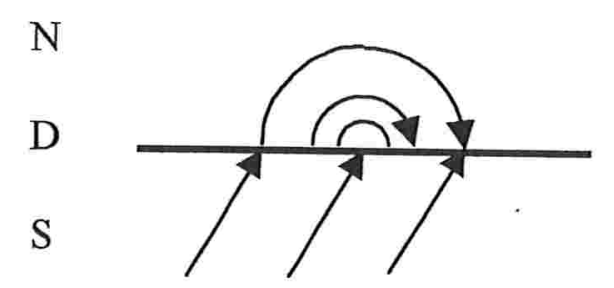

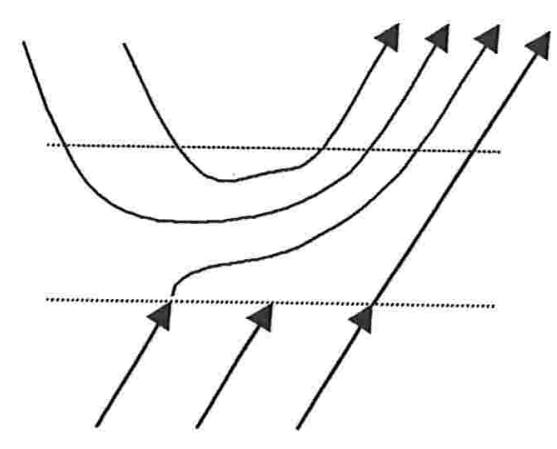

Fig. 39

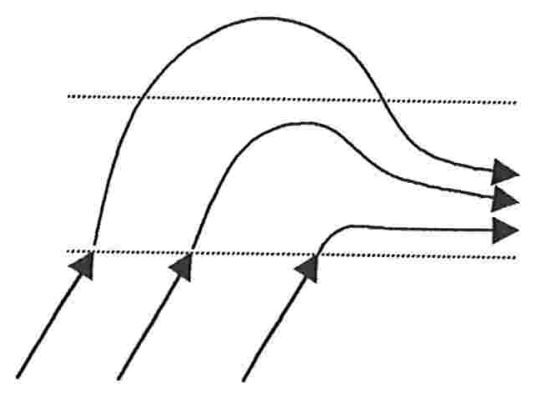

Fig. 41 
As figuras 40 e 41 exemplificam pontos de D-dobra de $Z$ e a sua regularização.

As Proposições 1.7, 1.8 e 1.9 tratam da regularização das politrajetórias fechadas tipos I, II e III, respectivamente. É verificado que nos três casos são geradas órbitas periódicas hiperbólicas. Merece destaque a Proposição 1.7, em cuja demonstração é estabelecida a derivada da aplicação de Poincaré, no contexto dos Campos Vetoriais Descontínuos. A Proposição 1.7 foi enunciada e as Proposições 1.8 e 1.9 foram estabelecidas por Sotomayor e Teixeira [S-T]. Os detalhes das demonstrações destas Proposições foram feitos neste trabalho.

\subsection{Proposição}

Seja $\gamma$ uma poli-trajetória fechada elementar tipo $I$ de $Z=(X, Y) \in \Omega^{r}(M, f), r \geq 1$. Então, dada uma função de transição $\varphi$, existem uma vizinhança $\vartheta$ de $\gamma$ em $M e \varepsilon_{0}>0$ tais que para cada $0<\varepsilon<\varepsilon_{0}, Z_{\varepsilon}$ contém uma única órbita periódica em $\vartheta$, e esta é hiperbólica.

\section{demonstração}

O primeiro passo será construir um anel em torno de $\gamma$ tal que as poli-trajetórias de $Z$ só estejam entrando ou saindo deste anel. Depois será mostrado que este anel implica na existência de um anel semelhante para $Z_{\varepsilon}$, se $\varepsilon$ é pequeno, no qual as órbitas de $Z_{\varepsilon}$ estarão apenas entrando ou saindo. O Teorema de Poincaré-Bendixon será usado para provar a existência de pelo menos uma órbita periódica de $Z_{\varepsilon}$. Por último, o fato de $\gamma$ ser uma poli-trajetória fechada elementar implicará que a órbita periódica de $Z_{\varepsilon}$ neste anel é única e hiperbólica.

Recorda-se que o sistema de coordenadas que está sendo utilizado é tal que $X(x, y)=(a(x, y), b(x, y)), Y(x, y)=(c(x, y), d(x, y))$ e $f(x, y)=y$.

\section{Logo,}

$$
\text { (1) } Z_{\varepsilon}(x, y)=\left(\left(1-\varphi_{\varepsilon}(y)\right) c(x, y)+\varphi_{\varepsilon}(y) a(x, y),\left(1-\varphi_{\varepsilon}(y)\right) d(x, y)+\varphi_{\varepsilon}(y) b(x, y)\right) \text {. }
$$

Pode-se considerar, sem perda de generalidade, que $\gamma$ é composta por apenas duas componentes, $\gamma_{0}$ e $\gamma_{1}$, como ilustra a figura 42. Esta consideração é feita com o objetivo de simplificar os cálculos, sem que isto interfira no resultado final. 


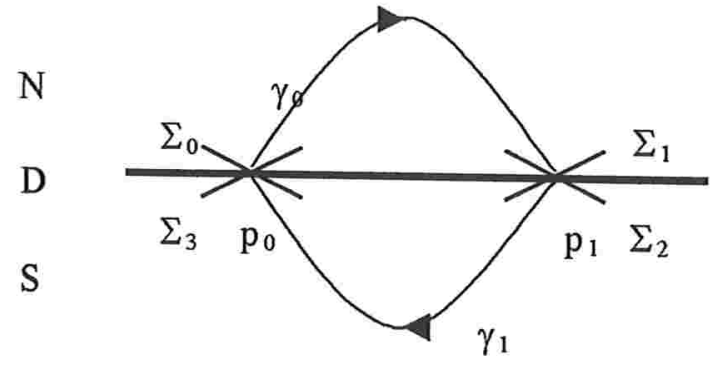

Fig. 42

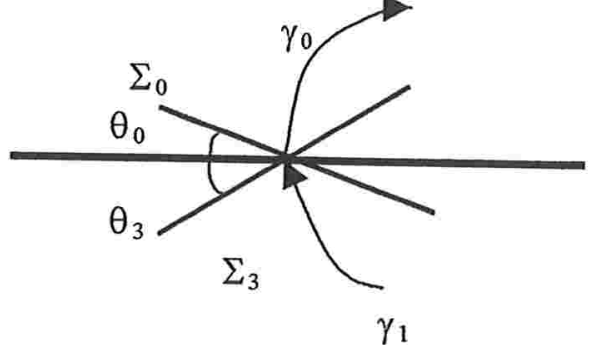

Fig. 43

$\operatorname{Sejam}\left\{\mathrm{p}_{0}, \mathrm{p}_{1}\right\}=\mathrm{D} \cap \gamma$

Sejam $\Sigma_{0}$ e $\Sigma_{1}$ seções ortogonais a $\gamma_{0}$ em $p_{0}$ e $p_{1}$, respectivamente e sejam $\Sigma_{2}$ e $\Sigma_{3}$ seções ortogonais a $\gamma_{1}$ em $p_{1}$ e $p_{0}$, como mostra a figura $4 \mathbf{2}$.

Sejam $\pi_{0}: \Sigma_{0} \rightarrow \Sigma_{1}$ e $\pi_{1}: \Sigma_{2} \rightarrow \Sigma_{3}$ as aplicações de Poincaré definidas pelo fluxo de $X$ e de $Y$, respectivamente.

Seja $\eta=\pi_{1}{ }^{\circ} \pi_{0}$

Sejam $\theta_{0}, \ldots, \theta_{3}$ os ângulos formados entre $\Sigma_{0}, \ldots, \Sigma_{3}$ e D, respectivamente.

Tem-se (ver [S2] e [A])

$\eta^{\prime}\left(\mathrm{p}_{0}\right)=\left[\pi_{1}{ }^{\mathrm{o}} \pi_{0}\left(\mathrm{p}_{0}\right)\right]^{\prime}=\pi_{1}{ }^{\prime}\left(\pi_{0}\left(\mathrm{p}_{0}\right)\right) \cdot \pi_{0}{ }^{\prime}\left(\mathrm{p}_{0}\right)=\pi_{1}{ }^{\prime}\left(\mathrm{p}_{1}\right) \cdot \pi_{0}{ }^{\prime}\left(\mathrm{p}_{0}\right)=$

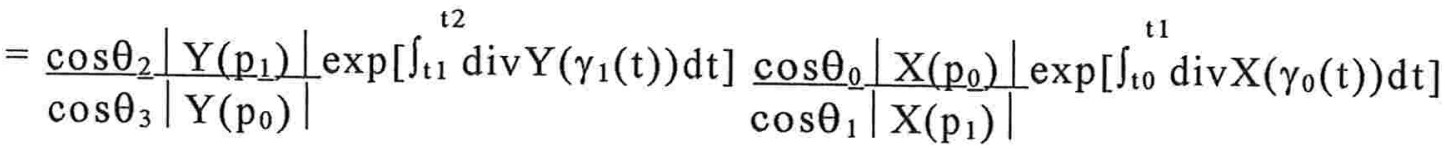

Pode-se supor, sem perda de generalidade, que $\eta^{\prime}\left(p_{0}\right)<1$.

Seja uma orientação qualquer em $\Sigma_{0}$, e fixemos a origem em $p_{0}$. Sejam $s_{0}$ e $s_{1} \in \Sigma_{0}$ tais que $s_{0}<0<s_{1}$. Como $\eta^{\prime}\left(p_{0}\right)<1$, então pode-se afirmar que $s_{0}<\eta\left(s_{0}\right)<0$ e $0<\eta\left(s_{1}\right)<s_{1}$.

Seja B um anel contendo $\gamma$ tal que B é formado pelas poli-trajetórias de $Z$ por $s_{0}$ e $s_{1}$ entre $\Sigma_{0}$ e $\eta\left(\Sigma_{0}\right)$ e pelos arcos $s_{0} \eta\left(s_{0}\right)$ e $\eta\left(s_{1}\right) s_{1}$ contidos em $\Sigma_{0}$. Pela construção, as poli-trajetórias de $Z$ através dos $\operatorname{arcos} s_{0} \eta\left(s_{0}\right)$ e $\eta\left(s_{1}\right) s_{1}$ em $\Sigma_{0}$ entram em B. Ver figura a seguir. 


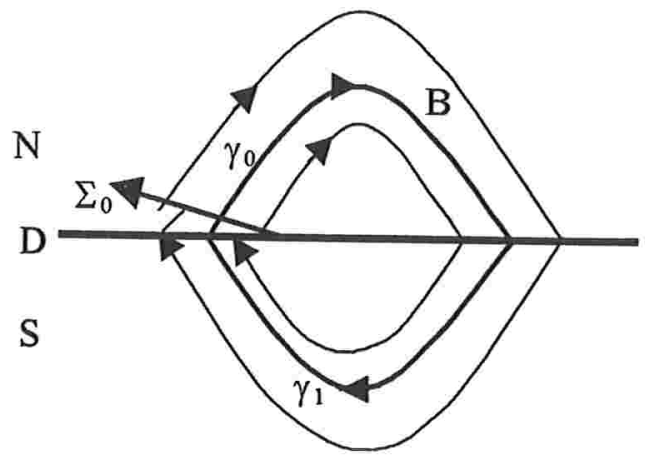

Fig. 44

Seja $\varepsilon_{0}>0$ pequeno o suficiente para que $\eta_{\varepsilon}\left(s_{0}\right)>s_{0}$ e $\eta_{\varepsilon}\left(s_{1}\right)<s_{1}$, onde $\eta_{\varepsilon}: \Sigma_{0} \rightarrow \Sigma_{0}$ é a aplicação de Poincaré do campo regularizado $Z_{\varepsilon}$, com $0<\varepsilon<\varepsilon_{0}$. A existência do número $\varepsilon_{0}>0$ pode ser comprovada pela continuidade das funções envolvidas.

Seja $B_{\varepsilon}$ o anel formado pelas órbitas de $Z_{\varepsilon}$ por $s_{0}$ e $s_{1}$, entre $\Sigma_{0}$ e $\eta_{\varepsilon}\left(\Sigma_{0}\right)$ e pelos $\operatorname{arcos} s_{0} \eta_{\varepsilon}\left(s_{0}\right)$ e $\eta_{\varepsilon}\left(s_{1}\right) s_{1}$ contidos em $\Sigma_{0}$. Pela construção, as órbitas de $Z_{\varepsilon}$ entram em $B_{\varepsilon}$ e não existem pontos singulares em $B_{\varepsilon}$. Pelo teorema de Poicaré-Bendixson, existe uma órbita periódica $\gamma_{\varepsilon}$ em $B_{\varepsilon}$. Resta mostrar que $\gamma_{\varepsilon}$ é hiperbólica.

Como $\eta_{\varepsilon}: \Sigma_{0} \rightarrow \Sigma_{0}$ é a aplicação de Poincaré de $Z_{\varepsilon}$, então

$\eta_{\varepsilon}^{\prime}\left(p_{\varepsilon}\right)=\exp \left[\int_{t 0 \varepsilon}^{t 1 \varepsilon} \operatorname{div} Z_{\varepsilon}\left(\gamma_{\varepsilon}(t)\right) d t\right]$.

Vale $\lim _{\varepsilon \rightarrow 0} \eta_{\varepsilon}^{\prime}\left(p_{\varepsilon}\right)=\eta^{\prime}\left(p_{0}\right)$.

De fato,

Seja $\gamma_{\varepsilon}(t)=\left(x_{\varepsilon}(t), y_{\varepsilon}(t)\right)$.

Então,

$$
\begin{aligned}
& \mathrm{Z}_{\varepsilon}\left(\gamma_{\varepsilon}(\mathrm{t})\right)=\left(\mathrm{Z}_{1 \varepsilon}, \mathrm{Z}_{2 \varepsilon}\right)\left(\gamma_{\varepsilon}(\mathrm{t})\right)= \\
& =\left(\left(1-\varphi_{\varepsilon}\left(\mathrm{y}_{\varepsilon}(\mathrm{t})\right)\right) \mathrm{c}\left(\mathrm{x}_{\varepsilon}(\mathrm{t}), \mathrm{y}_{\varepsilon}(\mathrm{t})\right)+\varphi_{\varepsilon}\left(\mathrm{y}_{\varepsilon}(\mathrm{t})\right) \mathrm{a}\left(\mathrm{x}_{\varepsilon}(\mathrm{t}), \mathrm{y}_{\varepsilon}(\mathrm{t})\right),\right. \\
& \left.\quad\left(1-\varphi_{\varepsilon}\left(\mathrm{y}_{\varepsilon}(\mathrm{t})\right)\right) \mathrm{d}\left(\mathrm{x}_{\varepsilon}(\mathrm{t}), \mathrm{y}_{\varepsilon}(\mathrm{t})\right)+\varphi_{\varepsilon}\left(\mathrm{y}_{\varepsilon}(\mathrm{t})\right) \mathrm{b}\left(\mathrm{x}_{\varepsilon}(\mathrm{t}), \mathrm{y}_{\varepsilon}(\mathrm{t})\right)\right) .
\end{aligned}
$$

Logo,

$\eta^{\prime}{ }_{\varepsilon}\left(p_{\varepsilon}\right)=\exp \left[\int_{t 0 \varepsilon}^{t 1 \varepsilon} \operatorname{div} Z_{\varepsilon}\left(\gamma_{\varepsilon}(t)\right) d t\right]=\exp \left[\int_{t 0 \varepsilon}^{t 1 \varepsilon}\left[(\partial / \partial x)\left(Z_{1 \varepsilon}\right)+(\partial / \partial y)\left(Z_{2 \varepsilon}\right)\right]\left(\gamma_{\varepsilon}(t)\right) d t\right]$ 


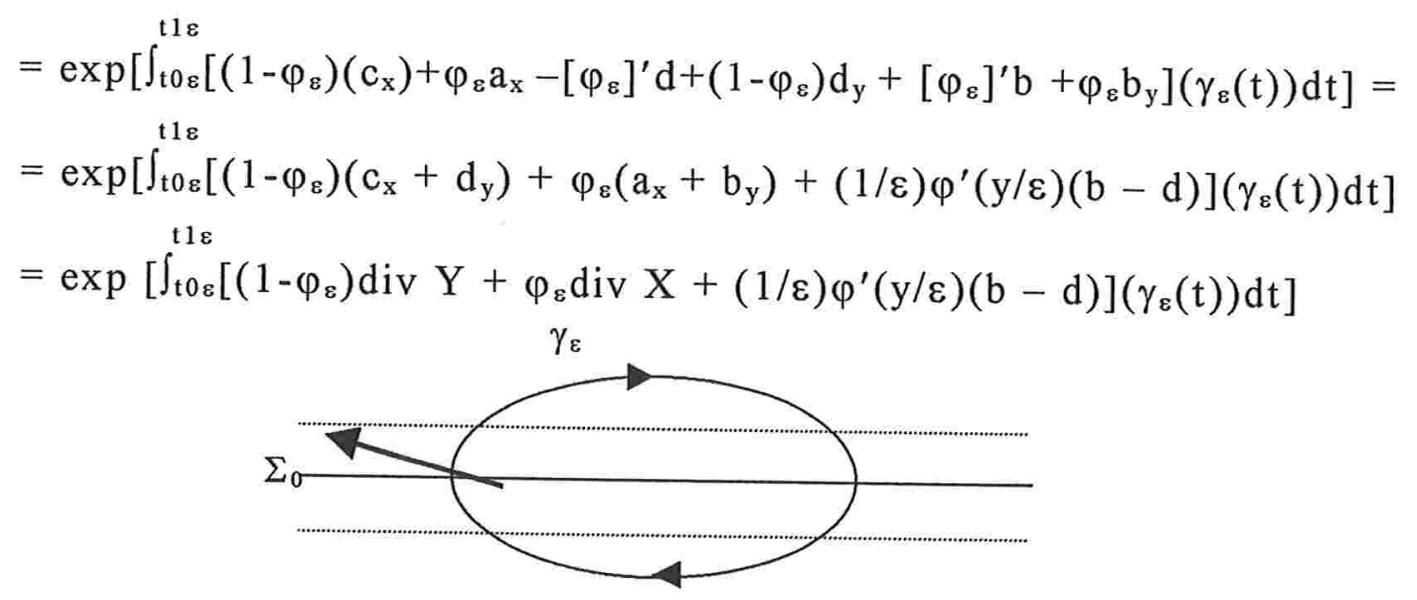

Fig. 45

Apenas no trecho em que $\gamma_{\varepsilon}$ está dentro da faixa de regularização tem-se a expressão completa de $\eta^{\prime}{ }_{\varepsilon}\left(p_{\varepsilon}\right)$ acima. Decompondo-se a expressão na soma das derivadas de $\eta_{\varepsilon}\left(p_{\varepsilon}\right)$ nos arcos definidos pela faixa de regularização, tem-se:

$\eta^{\prime}{ }_{\varepsilon}\left(p_{\varepsilon}\right)=\exp \left[\int_{t 0_{\varepsilon}}^{t^{\prime} \varepsilon}\left[\left(1-\varphi_{\varepsilon}\right) \operatorname{div} Y+\varphi_{\varepsilon} \operatorname{div} X+(1 / \varepsilon) \varphi^{\prime}(y / \varepsilon)(b-d)\right]\left(\gamma_{\varepsilon}(t)\right) d t+\right.$ $\int_{\mathrm{t}^{\prime} \varepsilon}^{\mathrm{t}^{\prime \prime} \varepsilon} \operatorname{div} \mathrm{X}\left(\gamma_{\varepsilon}(\mathrm{t})\right) \mathrm{dt}+\int_{\mathrm{t}^{\prime \prime} \varepsilon}^{\mathrm{t}^{\prime \prime \prime} \varepsilon}\left[\left(1-\varphi_{\varepsilon}\right) \operatorname{div} \mathrm{Y}+\varphi_{\varepsilon} \operatorname{div} \mathrm{X}+(1 / \varepsilon) \varphi^{\prime}(\mathrm{y} / \varepsilon)(\mathrm{b}-\mathrm{d})\right]\left(\gamma_{\varepsilon}(\mathrm{t})\right) \mathrm{dt}$ $\left.\int_{t^{\prime \prime \prime} \varepsilon}^{t(i v) \varepsilon} \operatorname{div} Y\left(\gamma_{\varepsilon}(t)\right) d t+\int_{t(i v) \varepsilon}^{t 1 \varepsilon}\left[\left(1-\varphi_{\varepsilon}\right) \operatorname{div} Y+\varphi_{\varepsilon} \operatorname{div} X+(1 / \varepsilon) \varphi^{\prime}(y / \varepsilon)(b-d)\right]\left(\gamma_{\varepsilon}(t)\right) d t\right]$ $\left.=\exp \left[\int_{t^{\prime} 0_{\varepsilon}}^{t^{\prime} \varepsilon}\left[\left(1-\varphi_{\varepsilon}\right) \operatorname{div} Y\right)\right]\left(\gamma_{\varepsilon}(t)\right) d t+\int_{t 0 \varepsilon}^{t^{\prime} \varepsilon}\left[\varphi_{\varepsilon} \operatorname{div} X\right]\left(\gamma_{\varepsilon}(t)\right) d t\right]$ $\exp \left[\int_{t 0 \varepsilon}^{t^{\prime} \varepsilon}\left[(1 / \varepsilon) \varphi^{\prime}(y / \varepsilon)(b-d)\right]\left(\gamma_{\varepsilon}(t)\right) d t\right] \exp \left[\int_{t^{\prime} \varepsilon}^{t^{\prime \prime} \varepsilon} \operatorname{div} X\left(\gamma_{\varepsilon}(t)\right) d t\right]$ $\exp \left[\int_{t^{\prime \prime} \varepsilon}^{t^{\prime \prime \prime} \varepsilon}\left[\left(1-\varphi_{\varepsilon}\right) \operatorname{div} Y\right]\left(\gamma_{\varepsilon}(t)\right) d t+\int_{t^{\prime \prime} \varepsilon}^{t^{\prime \prime \prime} \varepsilon}\left[\varphi_{\varepsilon} \operatorname{div} X\right]\left(\gamma_{\varepsilon}(t)\right) d t\right]$ $\exp \left[\int_{t^{\prime \prime} \varepsilon}^{t^{\prime \prime \prime} \varepsilon}\left[(1 / \varepsilon) \varphi^{\prime}(y / \varepsilon)(b-d)\right]\left(\gamma_{\varepsilon}(t)\right) d t\right] \exp \left[\int_{t^{\prime \prime \prime} \varepsilon}^{t(\text { iv }) \varepsilon} \operatorname{div} Y\left(\gamma_{\varepsilon}(t)\right) d t\right]$ $\exp \left[\int_{t(i v) \varepsilon}^{\mathrm{t} 1 \varepsilon}\left[\left(1-\varphi_{\varepsilon}\right) \operatorname{div} \mathrm{Y}\right]\left(\gamma_{\varepsilon}(\mathrm{t})\right) \mathrm{dt}+\int_{\mathrm{t}(\mathrm{iv}) \varepsilon}^{\mathrm{t} 1 \varepsilon}\left[\varphi_{\varepsilon} \operatorname{div} \mathrm{X}\right]\left(\gamma_{\varepsilon}(\mathrm{t})\right) \mathrm{dt}\right]$ $\exp \left[\int_{t(i v) \varepsilon}^{t 1 \varepsilon}\left[(1 / \varepsilon) \varphi^{\prime}(y / \varepsilon)(b-d)\right]\left(\gamma_{\varepsilon}(t)\right) d t\right]$

Onde $\left[\mathrm{t}_{0 \varepsilon}, \mathrm{t}^{\prime}{ }_{\varepsilon}\right]$ é o tempo para a órbita $\gamma_{\varepsilon}(\mathrm{t})$ ir de $\Sigma_{0}$ até sair da faixa de regularização, $\left[\mathrm{t}^{\prime}{ }_{\varepsilon}, \mathrm{t}^{\prime \prime}{ }_{\varepsilon}\right]$ é o tempo para $\gamma_{8}(\mathrm{t})$ permanecer na região $\mathrm{N}$ e fora da faixa de regularização, $\left[\mathrm{t}^{\prime \prime}{ }_{\varepsilon}, \mathrm{t}{ }^{\prime \prime \prime}{ }_{\varepsilon}\right]$ é o tempo para $\gamma_{\varepsilon}(\mathrm{t})$ atravessar novamente a faixa de regularização, $\left[\mathrm{t}^{\prime \prime \prime}{ }_{\varepsilon}, \mathrm{t}^{(\mathrm{iv})}{ }_{\varepsilon}\right]$ é o tempo para $\gamma_{\varepsilon}(\mathrm{t})$ permanecer na região $S$ e fora da faixa de regularização e, por último, $\left[\mathrm{t}^{(\mathrm{iv})}, \mathrm{t}_{1 \varepsilon}\right]$ é o tempo para $\gamma_{\varepsilon}(\mathrm{t})$ entrar na faixa de regularização e cruzar $\Sigma_{0}$. 
Tem-se

$\left.\lim _{\varepsilon \rightarrow 0} \exp \left[\int_{t 0 \varepsilon}^{t^{\prime} \varepsilon}\left[\left(1-\varphi_{\varepsilon}\right) \operatorname{div} Y\right)\right]\left(\gamma_{\varepsilon}(t)\right) d t+\int_{t 0 \varepsilon}^{t^{\prime} \varepsilon}\left[\varphi_{\varepsilon} \operatorname{div} X\right]\left(\gamma_{\varepsilon}(t)\right) d t\right]=1$.

O cálculo do limite acima foi feito levando-se em conta que os intervalos de integração estão tendendo a um ponto quando $\varepsilon$ tende a zero, e as funções integrando são funções limitadas, logo as integrais tendem a zero e portanto o limite tende a 1 .

Analogamente,

$\left.\left.\lim _{\varepsilon \rightarrow 0} \exp \left[\int_{t^{\prime \prime} \varepsilon}^{t^{\prime \prime \prime}[}\left[\left(1-\varphi_{\varepsilon}\right) \operatorname{div} Y\right)\right]\left(\gamma_{\varepsilon}(t)\right) d t+\int_{t^{\prime \prime}{ }_{\varepsilon}\left[\varphi_{\varepsilon}\right.}^{t^{\prime \prime \prime}{ }_{\varepsilon}} \operatorname{div} X\right]\left(\gamma_{\varepsilon}(t)\right) d t\right]=1$

e

$\left.\lim _{\varepsilon \rightarrow 0} \exp \left[\int_{t(\mathrm{iv}) \varepsilon}^{\mathrm{t} \varepsilon}\left[\left(1-\varphi_{\varepsilon}\right) \operatorname{div} \mathrm{Y}\right)\right]\left(\gamma_{\varepsilon}(\mathrm{t})\right) d t+\int_{\mathrm{t}(\mathrm{iv}) \varepsilon}^{\mathrm{t} \varepsilon}\left[\varphi_{\varepsilon} \operatorname{div} \mathrm{X}\right]\left(\gamma_{\varepsilon}(\mathrm{t})\right) \mathrm{dt}\right]=1$

Tem-se

$\lim _{\varepsilon \rightarrow 0} \exp \left[\int_{t^{\prime} \varepsilon}^{t^{\prime \prime} \varepsilon} \operatorname{div} X\left(\gamma_{\varepsilon}(t)\right) d t\right]=\exp \left[\int_{t 0}^{t 1} \operatorname{div} X\left(\gamma_{0}(t)\right) d t\right]$

$\mathrm{e}$

$\lim _{\varepsilon \rightarrow 0} \exp \left[\int_{t^{\prime \prime \prime} \varepsilon}^{t(i v) \varepsilon} \operatorname{div} Y\left(\gamma_{\varepsilon}(t)\right) d t\right]=\exp \left[\int_{t 1}^{t 2} \operatorname{div} Y\left(\gamma_{1}(t)\right) d t\right]$

Logo, para concluir que $\lim _{\varepsilon \rightarrow 0} \eta_{\varepsilon}^{\prime}\left(p_{\varepsilon}\right)=\eta^{\prime}\left(p_{0}\right)$ resta mostrar que

$\lim _{\varepsilon \rightarrow 0} \exp \left[\int_{t 0 \varepsilon}^{t^{\prime} \varepsilon}\left[(1 / \varepsilon) \varphi^{\prime}(y / \varepsilon)(b-d)\right]\left(\gamma_{\varepsilon}(t)\right) d t\right]$

$\exp \left[\int_{t^{\prime \prime} \varepsilon}^{t \prime \prime}\left[(1 / \varepsilon) \varphi^{\prime}(y / \varepsilon)(b-d)\right]\left(\gamma_{\varepsilon}(t)\right) d t\right]$

$\exp \left[\int_{\mathrm{t}(\mathrm{iv}) \varepsilon}^{\mathrm{t} 1 \varepsilon}\left[(1 / \varepsilon) \varphi^{\prime}(\mathrm{y} / \varepsilon)(\mathrm{b}-\mathrm{d})\right]\left(\gamma_{\varepsilon}(\mathrm{t})\right) \mathrm{dt}\right]=\frac{\cos \theta_{2}}{\cos \theta_{3}} \frac{\mathrm{Y}\left(\mathrm{p}_{1}\right) \mid}{\left|\mathrm{Y}\left(\mathrm{p}_{0}\right)\right|} \frac{\cos \theta_{0}}{\cos \theta_{1}} \frac{\left|\mathrm{X}\left(\mathrm{p}_{0}\right)\right|}{\left|\mathrm{X}\left(\mathrm{p}_{1}\right)\right|}$

No sistema de coordenadas utilizado tem-se

$\frac{\cos \theta_{2}}{\cos \theta_{3}} \frac{\left|\mathrm{Y}\left(\mathrm{p}_{1}\right)\right|}{\left|\mathrm{Y}\left(\mathrm{p}_{0}\right)\right|} \frac{\cos \theta_{0}}{\cos \theta_{1}} \frac{\mathrm{X}\left(\mathrm{p}_{0}\right) \mid}{\left|\mathrm{X}\left(\mathrm{p}_{1}\right)\right|}=$

$=\frac{-\mathrm{d}\left(\mathrm{p}_{1}\right) \cdot \mid}{\left|\mathrm{Y}\left(\mathrm{p}_{1}\right)\right|} \mid \frac{\mathrm{Y}\left(\mathrm{p}_{0}\right) \mid}{\mathrm{d}\left(\mathrm{p}_{0}\right)} \cdot \frac{\left|\mathrm{Y}\left(\mathrm{p}_{1}\right)\right|}{\left|\mathrm{Y}\left(\mathrm{p}_{0}\right)\right|} \cdot \frac{\mathrm{b}\left(\mathrm{p}_{0}\right)}{\mathrm{X}\left(\mathrm{p}_{0}\right) \mid} \cdot \frac{\left|\mathrm{X}\left(\mathrm{p}_{1}\right)\right|}{\left(-\mathrm{b}\left(\mathrm{p}_{1}\right)\right)} \cdot \frac{\left|\mathrm{X}\left(\mathrm{p}_{0}\right)\right|}{\left|\mathrm{X}\left(\mathrm{p}_{1}\right)\right|}$

$=\underline{\mathrm{d}\left(\mathrm{p}_{1}\right) \mathrm{b}\left(\mathrm{p}_{0}\right)}$

$\mathrm{d}\left(\mathrm{p}_{0}\right) \mathrm{b}\left(\mathrm{p}_{1}\right)$ 
De (1) tem-se

$$
\frac{d y}{d t}=\left(1-\varphi_{\varepsilon}(y)\right) d(x, y)+\varphi_{\varepsilon}(y) b(x, y) \Rightarrow \frac{d t}{d y}=\frac{1}{\left(1-\varphi_{\varepsilon}(y)\right) d(x, y)+\varphi_{\varepsilon}(y) b(x, y)}
$$

Supondo-se b e d constantes nos intervalos de integração considerados, segue

$$
\begin{aligned}
& \exp \left[\int_{t 0 \varepsilon}^{t^{\prime} \varepsilon}\left[(1 / \varepsilon) \varphi^{\prime}(y / \varepsilon)(b-d)\right]\left(\gamma_{\varepsilon}(t)\right) d t+\int_{t^{\prime \prime} \varepsilon}^{t^{\prime \prime \prime} \varepsilon}\left[(1 / \varepsilon) \varphi^{\prime}(y / \varepsilon)(b-d)\right]\left(\gamma_{\varepsilon}(t)\right) d t\right. \\
& \left.+\int_{\mathrm{t}(\mathrm{iv}) \varepsilon}^{\mathrm{t} 1 \varepsilon}\left[(1 / \varepsilon) \varphi^{\prime}(\mathrm{y} / \varepsilon)(\mathrm{b}-\mathrm{d})\right]\left(\gamma_{\varepsilon}(\mathrm{t})\right) \mathrm{dt}\right]= \\
& =\exp \left[\int_{t(\mathrm{iv}) \varepsilon}^{\mathrm{t}^{\prime} \varepsilon}\left[(1 / \varepsilon) \varphi^{\prime}(\mathrm{y} / \varepsilon)(\mathrm{b}-\mathrm{d})\right]\left(\gamma_{\varepsilon}(\mathrm{t})\right) \mathrm{dt}+\int_{\mathrm{t}^{\prime \prime} \varepsilon}^{\mathrm{t}^{\prime \prime \prime} \varepsilon}\left[(1 / \varepsilon) \varphi^{\prime}(\mathrm{y} / \varepsilon)(\mathrm{b}-\mathrm{d})\right]\left(\gamma_{\varepsilon}(\mathrm{t})\right) \mathrm{dt}\right] \\
& =\exp \left[\int_{-\varepsilon}^{\varepsilon} \frac{\left[(1 / \varepsilon) \varphi^{\prime}(y / \varepsilon)(\mathrm{b}-\mathrm{d})\right] d y}{\left(1-\varphi_{\varepsilon}(\mathrm{y})\right) \mathrm{d}+\varphi_{\varepsilon}(\mathrm{y}) \mathrm{b}}+\int_{\varepsilon}^{-\varepsilon} \frac{\left.\left[(1 / \varepsilon) \varphi^{\prime}(\mathrm{y} / \varepsilon)(\mathrm{b}-\mathrm{d})\right] d y\right]}{\left(1-\varphi_{\varepsilon}(\mathrm{y})\right) \mathrm{d}+\varphi_{\varepsilon}(\mathrm{y}) \mathrm{b}}\right. \\
& =\exp \left[\left.\ln \left(\left(1-\varphi_{\varepsilon}(y)\right) d+\varphi_{\varepsilon}(y) b\right)\right|_{-\varepsilon} ^{\varepsilon}+\left.\ln \left(\left(1-\varphi_{\varepsilon}(y)\right) d+\varphi_{\varepsilon}(y) b\right)\right|_{\varepsilon} ^{-\varepsilon}\right] \\
& =\exp \left[\ln \left(\left(1-\varphi_{\varepsilon}(\varepsilon)\right) d\left(\mathrm{p}_{0}\right)+\varphi_{\varepsilon}(\varepsilon) b\left(\mathrm{p}_{0}\right)\right)-\ln \left(\left(1-\varphi_{\varepsilon}(-\varepsilon)\right) d\left(\mathrm{p}_{0}\right)+\varphi_{\varepsilon}(-\varepsilon) b\left(\mathrm{p}_{0}\right)\right)+\right. \\
& \left.+\ln \left(\left(1-\varphi_{\varepsilon}(-\varepsilon)\right) d\left(p_{1}\right)+\varphi_{\varepsilon}(-\varepsilon) b\left(p_{1}\right)\right)-\ln \left(\left(1-\varphi_{\varepsilon}(\varepsilon)\right) d\left(p_{1}\right)+\varphi_{\varepsilon}(\varepsilon) b\left(p_{1}\right)\right)\right]= \\
& =\exp \left[\ln \left(\mathrm{b}\left(\mathrm{p}_{0}\right)\right)-\ln \left(\mathrm{d}\left(\mathrm{p}_{0}\right)\right)+\ln \left(\mathrm{d}\left(\mathrm{p}_{1}\right)\right)-\ln \left(\mathrm{b}\left(\mathrm{p}_{1}\right)\right)\right]= \\
& =\exp \ln \left(\mathrm{b}\left(\mathrm{p}_{0}\right)\right) \exp \left(-\ln \left(\mathrm{d}\left(\mathrm{p}_{0}\right)\right)\right) \exp \ln \left(\mathrm{d}\left(\mathrm{p}_{1}\right)\right) \exp \left(-\ln \left(\mathrm{b}\left(\mathrm{p}_{1}\right)\right)=\right. \\
& =\underline{\mathrm{b}}\left(\mathrm{p}_{0}\right) \mathrm{d}\left(\underline{p}_{1}\right) \\
& \mathrm{d}\left(\mathrm{p}_{0}\right) \mathrm{b}\left(\mathrm{p}_{1}\right)
\end{aligned}
$$

Os cálculos acima valem em intervalos de integração em que b e d podem ser considerados constantes, o que não é uma suposição restritiva quando $\varepsilon$ tende a zero, logo, está demostrado que

$$
\lim _{\varepsilon \rightarrow 0} \eta_{\varepsilon}^{\prime}\left(p_{\varepsilon}\right)=\eta^{\prime}\left(p_{0}\right)<1
$$

Logo, diminuindo-se $\varepsilon_{0}>0$, se necessário, tem-se $\eta^{\prime}{ }_{\varepsilon}\left(\mathrm{p}_{\varepsilon}\right)<1$ para qualquer $0<\varepsilon<\varepsilon_{0}$, e $\gamma_{\varepsilon}$ será uma órbita periódica hiperbólica atratora para o campo regularizado $Z_{\varepsilon}$. Analogamente, se $\eta^{\prime}\left(p_{0}\right)>1$, existe $\varepsilon_{0}>0$ tal que $\eta^{\prime}{ }_{\varepsilon}\left(\mathrm{p}_{\varepsilon}\right)<1$ para qualquer $0<\varepsilon<\varepsilon_{0}$, e $\gamma_{\varepsilon}$ será uma órbita periódica hiperbólica repulsora para o campo $Z_{\varepsilon}$. 


\subsection{Proposição}

Seja $\gamma$ uma poli-trajetória fechada elementar tipo II de $Z=(X, Y) \in \Omega^{r}(M, f), r \geq 1$. Então, dada uma função de transição $\varphi$, existem uma vizinhança $\vartheta$ de $\gamma$ em $M$ e $\varepsilon_{0}>0$ tal que para cada $0<\varepsilon<\varepsilon_{0}, Z_{\varepsilon}$ contém uma única órbita periódica em $\vartheta$, e esta é hiperbólica.

demonstração

Pode-se assumir, sem perda de generalidade, que D = DES, isto é, todos os pontos de $\mathrm{D}$ pertencem ao arco de deslizamento.

Sejam consideradas coordenadas polares $(\theta, \rho)$ em torno de D em $M$ de modo que $\mathrm{D}=\{\rho=0,0 \leq \theta \leq 2 \pi\}$. Neste caso, $f(\theta, \rho)=\rho$.

Seja $Z(\theta, \rho)=(X(\theta, \rho), Y(\theta, \rho))$, onde $X(\theta, \rho)=(a(\theta, \rho), b(\theta, \rho))$ e $Y(\theta, \rho)=(c(\theta, \rho), d(\theta, \rho))$.

Afirma-se que existe $\varepsilon_{0}>0$ tal que se $0<\varepsilon<\varepsilon_{0}$, então o campo vetorial regularizado

$Z_{\varepsilon}(\theta, \rho)=\left(Z_{\varepsilon}{ }^{1}(),. Z_{\varepsilon}{ }^{2}().\right)=\left(\left(1-\varphi_{\varepsilon}(\rho)\right) c()+.\varphi_{\varepsilon}(\rho) a(),.\left(1-\varphi_{\varepsilon}(\rho)\right) d()+.\varphi_{\varepsilon}(\rho) b().\right)$

possui uma órbita periódica $\gamma_{\varepsilon}=\left\{\left(\theta, \rho_{\varepsilon}(\theta)\right): 0 \leq \theta \leq 2 \pi\right\}$ em $\vartheta=\left\{(\theta, \rho):|\rho| \leq \varepsilon_{0}\right\}$.

De fato, como os pontos de $\mathrm{D}$ pertencem ao arco tipo deslizante, $\mathrm{X} f(\theta, 0)=\mathrm{b}(\theta, 0)<0$ e $\mathrm{Y} f(\theta, 0)=\mathrm{d}(\theta, 0)>0$. Por continuidade, pode-se tomar $\varepsilon_{0}>0$ tal que $\mathrm{b}(\theta, \rho)<0$ e $\mathrm{d}(\theta, \rho)>0$ se $|\rho| \leq \varepsilon_{0}$. Portanto, se $0<\varepsilon<\varepsilon_{0}$, todas as órbitas do campo $Z_{\varepsilon}$ estão entrando em $\vartheta$ e pelo Teorema de Poincaré-Bendixson existe pelo menos uma órbita periódica em $\vartheta$ (o Corolário 1.2 exclui a possibilidade de haver uma singularidade em $\vartheta$ ).

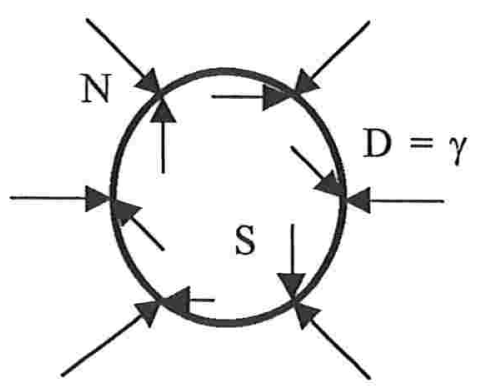

Fig. 46

A figura 46 representa uma poli-trajetória fechada tipo II. 
Seja $\gamma_{\varepsilon}$ uma órbita periódica de $Z_{\varepsilon}$ em $\vartheta$.

Será provado que $\gamma_{\varepsilon}$ é atratora e hiperbólica, e portanto única.

Sejam $\mathrm{p} \in \gamma_{\varepsilon}, \sum$ uma seção transversal à órbita $\gamma_{\varepsilon}$ em p e $\pi: \sum_{0} \rightarrow \sum$ a transformação de Poincaré, onde $\Sigma_{0} \subset \sum$ é o domínio de $\pi$.

Pela Teoria de Equações Diferenciais [S1] tem-se

$\pi^{\prime}(\mathrm{p})=\exp \left[\int_{0}^{2 \pi} \operatorname{div} Z_{\varepsilon}\left(\gamma_{\varepsilon}(\theta)\right) \mathrm{d} \theta\right]$, onde $\operatorname{div} Z_{\varepsilon}=Z_{\varepsilon \theta}^{1}+Z_{\varepsilon p}^{2}$.

Será provado que $0<\pi^{\prime}(\mathrm{p})<1$.

De fato,

$\operatorname{div} Z_{\varepsilon}=\left(1-\varphi_{\varepsilon}\right) c_{\theta}+\varphi_{\varepsilon} a_{\theta}-\varphi_{\varepsilon}{ }^{\prime} d+\left(1-\varphi_{\varepsilon}\right) d_{\rho}+\varphi_{\varepsilon}{ }^{\prime} b+\varphi_{\varepsilon} b_{\rho}=$

$=-\varphi_{\varepsilon}{ }^{\prime}(d-b)+\varphi_{\varepsilon}\left(a_{\theta}+b_{\rho}\right)+\left(1-\varphi_{\varepsilon}\right)\left(c_{\theta}+d_{\rho}\right)=-\varphi_{\varepsilon}{ }^{\prime}(d-b)+L$,

onde $\mathrm{L}=\varphi_{\varepsilon} \operatorname{div} \mathrm{X}+\left(1-\varphi_{\varepsilon}\right) \operatorname{div} \mathrm{Y}$ é uma função limitada em $\vartheta$.

É imediato que $(\mathrm{d}-\mathrm{b})>0$ em $\gamma_{\varepsilon}$, portanto resta mostrar que $\varphi_{\varepsilon}^{\prime}\left(\rho_{\varepsilon}(\theta)\right) \rightarrow+\infty$ quando $\varepsilon \rightarrow 0, \forall \theta \in[0,2 \pi]$.

Para isto será considerado inicialmente um sistema simplificado no qual os campos X e Y são dados por

$$
\left\{\begin{array}{l}
X(\theta, \rho)=\left(1, b_{0}\right), \\
Y(\theta, \rho)=(0,1),
\end{array} \quad \text { onde } b_{0}<0\right. \text { é constante. }
$$

Neste caso, o campo vetorial regularizado fica

$$
Z_{\varepsilon}(\theta, \rho)=\left(\varphi_{\varepsilon}(\rho), 1-\varphi_{\varepsilon}(\rho)+\varphi_{\varepsilon}(\rho) b_{0}\right) .
$$

Fazendo a mudança de coordenadas $\rho^{*}=\rho / \varepsilon$ e $\theta *=\theta / \varepsilon$, tem-se

$$
\frac{d \rho^{*}}{d \theta^{*}}=\frac{1-\varphi\left(\rho^{*}\right)+\varphi\left(\rho^{*}\right)}{\varphi\left(\rho^{*}\right)} b_{0}
$$

Igualando esta última expressão a zero, encontra-se uma solução periódica do sistema, uma vez que o mesmo não depende de $\theta$.

Esta solução periódica é dada implicitamente por

$$
\varphi\left(\rho_{0}{ }^{*}\right)=\frac{1}{1-b_{0}}
$$


Como $b_{0}<0$,

$$
0<\varphi\left(\rho_{0}^{*}\right)=\frac{1}{1-b_{0}}<1
$$

A solução periódica $\rho_{0}{ }^{*}=\varphi^{-1}\left(1 /\left(1-b_{0}\right)\right)$ é única porque $\varphi$ é monótona.

Tem-se para $\varepsilon>0, \rho_{0}=\varepsilon \rho_{0}{ }^{*}, \log o$,

$$
\varphi_{\varepsilon}^{\prime}\left(\rho_{0}\right)=\left[\varphi\left(\rho_{0} / \varepsilon\right)\right]^{\prime}=(1 / \varepsilon) \varphi^{\prime}\left(\rho_{0} / \varepsilon\right)=(1 / \varepsilon) \varphi^{\prime}\left(\rho_{0}{ }^{*}\right) .
$$

Conforme $\varepsilon$ decresce, $\varphi_{\varepsilon}^{\prime}\left(\rho_{0}\right)$ aumenta. Isto se deve ao fato que $\varphi^{\prime}\left(\rho_{0}{ }^{*}\right)$ é constante (pois $\rho_{0}{ }^{*}$ não depende de $\varepsilon$ ) e estritamente positiva (pois $0<\varphi\left(\rho_{0}{ }^{*}\right)<1$ ), logo pode-se diminuir $\varepsilon$ o quanto for necessário de modo a se ter a derivada grande quanto for preciso.

(1) Com isto tem-se que $\rho=\rho_{0}=\varepsilon \rho_{0}{ }^{*}$ é uma primeira aproximação da solução periódica procurada. Será mostrado que pode-se tomar $\delta>0$ de modo que a solução do caso geral em que os campos são dados por $X(\theta, \rho)$ $=(\mathrm{a}(\theta, \rho), \mathrm{b}(\theta, \rho)), \mathrm{Y}(\theta, \rho)=(0,1)$ está no intervalo $\varepsilon\left(\rho_{0}{ }^{*} \pm \delta\right)$. Então, diminuindo-se $\varepsilon$ o quanto for necessário para que a derivada seja grande neste intervalo, implicará que a órbita periódica será hiperbólica.

Como os pontos da poli-trajetória são regulares, $\operatorname{det}[\mathrm{X}, \mathrm{Y}](\mathrm{p}) \neq 0$, para qualquer $\mathrm{p} \in \mathrm{D}$.

$$
\operatorname{det}[X, Y](p)=a(p) 1-b(p) \cdot 0=a(p) \neq 0
$$

Pode-se considerar, sem perda de generalidade, que $a(p)>0$. Como $\mathrm{D}$ é compacto, tem-se $\mathrm{k}=\min \{\mathrm{a}(\mathrm{p}) \mid \mathrm{p} \in \mathrm{D}\}>0$.

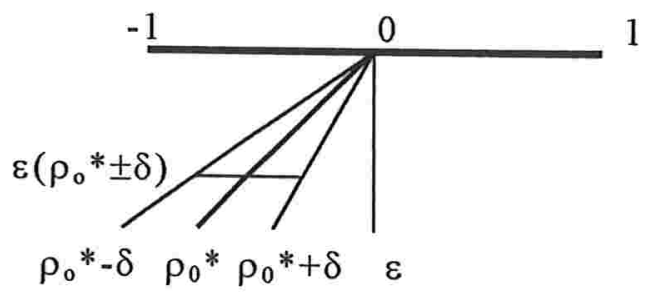

Fig. 47

O campo vetorial regularizado é dado por

$Z_{\varepsilon}(\theta, \rho)=\left(\varphi_{\varepsilon}(\rho) a(\theta, \rho),\left(1-\varphi_{\varepsilon}(\rho)\right)+\varphi_{\varepsilon}(\rho) b(\theta, \rho)\right)$ 

tem-se

Calculando-se a segunda coordenada de $Z_{\varepsilon}$ em $\left(\theta, \rho=\varepsilon\left(\rho_{0}{ }^{*} \pm \delta\right)\right)$,

$$
Z_{\varepsilon}^{2}\left(\theta, \varepsilon\left(\rho_{0}{ }^{*} \pm \delta\right)\right)=1-\varphi\left(\rho_{0}^{*} \pm \delta\right)+\varphi\left(\rho_{0}^{*} \pm \delta\right) b\left(\theta, \varepsilon\left(\rho_{0}^{*} \pm \delta\right)\right)
$$

Se for mostrado que $Z_{\varepsilon}^{2}\left(\theta, \varepsilon\left(\rho_{0}{ }^{*}+\delta\right)\right)<0$ e $Z^{2}{ }_{\varepsilon}\left(\theta, \varepsilon\left(\rho_{0}{ }^{*}-\delta\right)\right)>0$, terá sido provado que as órbitas entram no anel $\left(\theta, \rho=\varepsilon\left(\rho_{0}{ }^{*} \pm \delta\right)\right)$, e por Poincaré-Bendixson, a órbita periódica estará neste anel.

As expansões de b e $\varphi$ em série são dadas por

$$
\begin{aligned}
& \mathrm{b}(\theta, \rho)=b_{0}+b_{1}(\theta) \rho+b_{2}(\theta, \rho) \rho^{2} \\
& b\left(\theta, \varepsilon\left(\rho_{0}{ }^{*} \pm \delta\right)\right)=b_{0}+b_{1}(\theta) \varepsilon\left(\rho_{0}{ }^{*} \pm \delta\right)+b_{2}\left(\theta, \varepsilon\left(\rho_{0}{ }^{*} \pm \delta\right)\right) \varepsilon^{2}\left(\rho_{0}{ }^{*} \pm \delta\right)^{2} \\
& \varphi\left(\rho_{0}{ }^{*} \pm \delta\right)=\varphi\left(\rho_{0}{ }^{*}\right) \pm \varphi^{\prime}\left(\rho_{0}{ }^{*}\right) \delta+\frac{\varphi^{\prime \prime}\left(\rho_{0}{ }^{*}\right) \delta^{2}}{2}+r_{3}(\delta),
\end{aligned}
$$

onde $r_{3}(\delta)=\varphi^{\prime \prime \prime}\left(\rho_{0}^{*} \pm \mathrm{m} \delta\right), 0<\mathrm{m}<1$.

Logo,

$$
\begin{aligned}
& Z_{\varepsilon}^{2}\left(\theta, \varepsilon\left(\rho_{0}{ }^{*}+\delta\right)\right)=1-\left[\varphi\left(\rho_{0}{ }^{*}\right)+\varphi^{\prime}\left(\rho_{0}{ }^{*}\right) \delta+\frac{\varphi^{\prime \prime}\left(\underline{\rho}_{0}{ }^{*}\right) \delta^{2}}{2}+r_{3}(\delta)\right] . \\
& \left(1-b_{0}-b_{1}(\theta) \varepsilon\left(\rho_{0}{ }^{*}+\delta\right)-b_{2}(\theta, \rho) \varepsilon^{2}\left(\rho_{0}{ }^{*}+\delta\right)^{2}\right)= \\
& =1-\left[\frac{1}{1-b_{0}}+\varphi^{\prime}\left(\rho_{0}{ }^{*}\right) \delta+\frac{\varphi^{\prime \prime}\left(\rho_{0}{ }^{*}\right) \delta^{2}}{2}+r_{3}(\delta)\right] . \\
& \left(1-b_{0}-\varepsilon\left(\rho_{0}{ }^{*}+\delta\right)\left(b_{1}(\theta)+b_{2}(\theta, \rho) \varepsilon\left(\rho_{0}{ }^{*}+\delta\right)\right)\right)
\end{aligned}
$$

Fixado $\delta>0$, pode-se diminuir $\varepsilon>0$ de modo que o fator $\varepsilon\left(\rho_{0}{ }^{*}+\delta\right)\left(b_{1}(\theta)+b_{2}(\theta, \rho) \varepsilon\left(\rho_{0}{ }^{*}+\delta\right)\right)$ deixe de ser relevante e tenha-se

$$
\begin{aligned}
& {\left[\frac{1}{1-b_{0}}+\varphi^{\prime}\left(\rho_{0}^{*}\right) \delta+\frac{\varphi^{\prime \prime}\left(\rho_{0}{ }^{*}\right) \delta^{2}}{2}+r_{3}(\delta)\right]\left(1-b_{0}\right)} \\
& =1+\left[\varphi^{\prime}\left(\rho_{0}{ }^{*}\right) \delta+\frac{\varphi^{\prime \prime}\left(\rho_{0}{ }^{*}\right) \delta^{2}}{2}+r_{3}(\delta)\right]\left(1-b_{0}\right)>1 \\
& \text { e portanto } \\
& Z^{2}{ }_{\varepsilon}\left(\theta, \varepsilon\left(\rho_{0}{ }^{*}+\delta\right)\right)<0 .
\end{aligned}
$$$$
\text { Agora calculando } \mathrm{Z}_{\varepsilon}^{2}\left(\theta, \varepsilon\left(\rho_{0}{ }^{*}-\delta\right)\right)
$$$$
Z^{2}{ }_{\varepsilon}\left(\theta, \varepsilon\left(\rho_{0}{ }^{*}-\delta\right)\right)=1-\left[\frac{1}{1-b_{0}}-\varphi^{\prime}\left(\rho_{0}{ }^{*}\right) \delta+\frac{\varphi^{\prime \prime}\left(\rho_{0}{ }^{*}\right) \delta^{2}}{2}+r_{3}(\delta)\right] \text {. }
$$$$
\left(1-b_{0}-\varepsilon\left(\rho_{0}{ }^{*}-\delta\right)\left(b_{1}(\theta)+b_{2}(\theta, \rho) \varepsilon\left(\rho_{0}{ }^{*}-\delta\right)\right)\right)=
$$ 


$$
\begin{aligned}
& =1-\frac{1-b_{0}}{1-b_{0}}+\frac{\varepsilon\left(\rho_{0}{ }^{*}-\delta\right)\left(b_{1}(\theta)+b_{2}(\theta, \rho) \varepsilon\left(\rho_{0}{ }^{*}-\delta\right)\right)}{1-b_{0}}+\left(1-b_{0}\right) \varphi^{\prime}\left(\rho_{0}{ }^{*}\right) \delta+ \\
& -\varepsilon\left(\rho_{0}{ }^{*}-\delta\right)\left(b_{1}(\theta)+b_{2}(\theta, \rho) \varepsilon\left(\rho_{0}{ }^{*}-\delta\right)\right) \varphi^{\prime}\left(\rho_{0}{ }^{*}\right) \delta-\left(1-\underline{b}_{0}\right) \varphi^{\prime \prime}\left(\underline{\rho}_{0}{ }^{*}\right) \delta^{2}+ \\
& +\varepsilon\left(\rho_{0}{ }^{*}-\delta\right) \cdot\left(\underline{b}_{1}(\theta)+\underline{b}_{2}(\theta, \rho) \varepsilon\left(\underline{\rho}_{0}{ }^{*}-\delta\right)\right) \varphi^{\prime \prime}\left(\rho_{0}{ }^{*}\right) \delta^{2}+ \\
& 2 \\
& -r_{3}(\delta)\left(1-b_{0}-\varepsilon\left(\rho_{0}{ }^{*}-\delta\right)\left(b_{1}(\theta)+b_{2}(\theta, \rho) \varepsilon\left(\rho_{0}{ }^{*}-\delta\right)\right)\right)= \\
& =\varepsilon\left[\left(\underline{\rho}_{0}{ }^{*}-\delta\right)\left(b_{1}(\theta)+b_{2}(\theta, \rho) \varepsilon\left(\underline{\rho}_{0}{ }^{*}-\delta\right)\right)-\left(\rho_{0}{ }^{*}-\delta\right)\left(b_{1}(\theta)+b_{2}(\theta, \rho) \varepsilon\left(\rho_{0}{ }^{*}-\delta\right)\right)\right. \\
& \left.\varphi^{\prime}\left(\rho_{0}{ }^{*}\right) \delta-\left(\rho_{0}{ }^{*}-\delta\right)\left(b_{1}(\theta)+b_{2}(\theta, \rho) \varepsilon\left(\rho_{0}{ }^{*}-\delta\right)\right) \frac{\left.\varphi^{\prime \prime}\left(\rho_{0}{ }^{*}\right) \delta^{2}\right)}{2}\right]-\left(1-b_{0}\right)\left(\varphi^{\prime}\left(\rho_{0}{ }^{*}\right) \delta\right. \\
& \left.-\frac{\varphi^{\prime \prime}\left(\rho_{0}{ }^{*}\right) \delta^{2}}{2}\right)-r_{3}(\delta)\left(1-b_{0}-\varepsilon\left(\rho_{0}^{*}-\delta\right)\left(b_{1}(\theta)+b_{2}(\theta, \rho) \varepsilon\left(\rho_{0}^{*}-\delta\right)\right)\right) .
\end{aligned}
$$

Como $\delta^{2}<\delta<1$, pode-se tomar $\delta$ pequeno o suficiente para que

$$
\left(1-b_{0}\right)\left(\varphi^{\prime}\left(\rho_{0}{ }^{*}\right) \delta-\frac{\varphi^{\prime \prime}\left(\rho_{0}{ }^{*}\right) \delta^{2}}{2}>0\right. \text {. }
$$

Depois de fixado o valor de $\delta$ pode-se diminuir o valor de $\varepsilon$ de maneira que $Z^{2}\left(\theta, \varepsilon\left(\rho_{0}{ }^{*}-\delta\right)\right)>0$.

$O$ valor de $\delta$ pode ser tomado único e o valor de $\varepsilon_{0}>0$ pode ser fixado de modo que para $0<\varepsilon<\varepsilon_{0}$ tenha-se $Z^{2}{ }_{\varepsilon}\left(\theta, \varepsilon\left(\rho_{0}{ }^{*}+\delta\right)\right)<0$, $Z_{\varepsilon}^{2}\left(\theta, \varepsilon\left(\rho_{0}{ }^{*}-\delta\right)\right)>0$ e a derivada $\varphi_{\varepsilon}{ }^{\prime}\left(\rho_{0}\right)$ seja grande o suficiente para que a órbita periódica seja hiperbólica, conforme foi argumentado em (1).

\subsection{Proposição}

Seja $\gamma$ uma poli-trajetória fechada elementar tipo III de $Z=(X, Y) \in \Omega^{r}(M, f), r \geq 1$. Então, dada uma função de transição $\varphi$, existem uma vizinhança $\vartheta$ de $\gamma$ em $M$ e $\varepsilon_{0}>0$ tal que para cada $0<\varepsilon \leq \varepsilon_{0}$, $Z_{\varepsilon}$ contém uma única órbita periódica em $\vartheta$, e esta é hiperbólica.

demonstração

Seja $\gamma$ uma poli-trajetória fechada elementar tipo III de Z. Será considerado, sem perda de generalidade, que $\gamma$ possui um único arco de trajetória de $\mathrm{X}$ e um único arco de trajetória de $\mathrm{F}_{Z}$, e que este arco é de deslizamento. Consideram-se coordenadas tais que $f(\mathrm{x}, \mathrm{y})=\mathrm{y}$. Sejam $\Sigma_{1}$, $\Sigma_{2}, \Sigma_{3}$ e $\Sigma_{4}$ seções transversais à poli-trajetória $\gamma$, como ilustrado abaixo. 
Considera-se que as seções transversais foram escolhidas de modo que as órbitas de $\mathrm{X}$ que passam por $\Sigma_{3}$ entram no arco deslizante após passar por $\Sigma_{4}$ e depois cruzam $\Sigma_{1}$. Também será considerado que as órbitas que cruzam $\Sigma_{1}$ entram no arco de deslizamento.

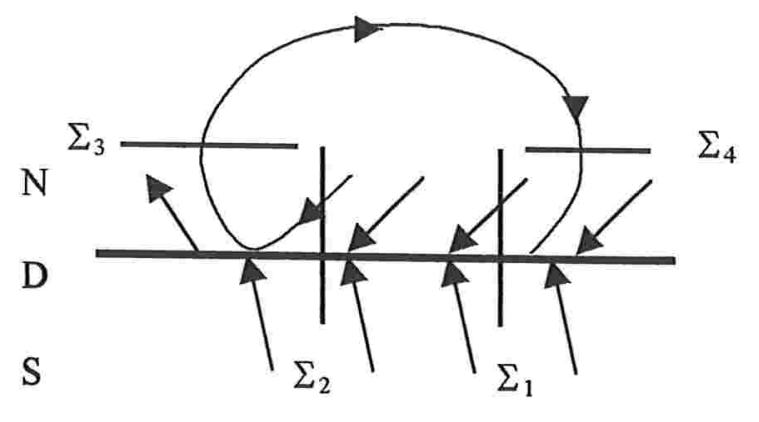

Fig. 48

A transformação de Poincaré entre cada seção $\Sigma$ será estudada separadamente. Será mostrado que no trecho entre $\Sigma_{1}$ e $\Sigma_{2}$ há uma aproximação das órbitas do campo regularizado, e no trecho entre $\Sigma_{2}$ e $\Sigma_{3}$ há um afastamento entre as órbitas, porém a expansão não é tão forte quanto a contração, e a órbita periódica hiperbólica será atratora.

Afirma-se que existe um anel B em torno de $\gamma$ e $\varepsilon_{0}>0$ tal que se $0<\varepsilon<\varepsilon_{0}$, então as órbitas de $Z_{\varepsilon}$ estão entrando em B.

De fato, seja $p_{3} \in \Sigma_{3} \cap \gamma$, e consideremos uma orientação em $\Sigma_{3}$. Sejam $\mathrm{q}_{0}$ e $\mathrm{q}_{1} \in \Sigma_{3}$ tais que $\mathrm{q}_{0}<\mathrm{p}_{3}<\mathrm{q}_{1}$ e as órbitas de $X$ por $\mathrm{q}_{0}$ e $\mathrm{q}_{1}$ cruzam $\Sigma_{4}$, como ilustra a figura 49 . Se $q_{0}$ e $q_{1}$ forem tomados suficientemente próximos a $\mathrm{p}_{3}$, então a órbita por $\mathrm{q}_{1}$ entrará no arco deslizante após cruzar a seção transversal $\Sigma_{4}$ e a órbita por $q_{1}$ cruzará a seção transversal $\Sigma_{1}$. Pela maneira como foi escolhida $\Sigma_{1}$, é claro que a órbita por $\mathrm{q}_{1}$ também entrará no arco deslizante. Sejam $\mathrm{q}_{3}=\left(\mathrm{m}_{3}, \mathrm{n}_{3}\right)$ o ponto da órbita de $X$ por $q_{1}$ quando a mesma cruza a seção $\Sigma_{2}$ e $\mathrm{q}_{4}=\left(\mathrm{m}_{4}, \mathrm{n}_{4}\right)$ o ponto quando cruza a seção $\Sigma_{1}$. Seja $\mathrm{n}_{5}<0$ de maneira que as órbitas de $\mathrm{Y}$ por $(\mathrm{x}, \mathrm{y})$ onde $0>\mathrm{y}>\mathrm{n}_{5}$ entram no arco deslizante. Seja $\mathrm{B}$ o anel limitado por um lado pela órbita por $q_{1}$ entre $\Sigma_{2}$ e $\Sigma_{1}$, pelo arco entre $\mathrm{x}=\mathrm{m}_{4}$ e $\mathrm{x}=\mathrm{m}_{3}$ contido em $\Sigma_{1}$ e pela reta $\mathrm{y}=\mathrm{n}_{6}$ entre $\Sigma_{1}$ e $\Sigma_{2}$, onde $\mathrm{n}_{6}=\min \left\{\mathrm{n}_{3}, \mathrm{n}_{4}\right\}$; e pelo outro lado pela órbita de X por q entre $\mathrm{D}$ e $\Sigma_{1}$, passando por $\Sigma_{3}$ e $\Sigma_{4}$, pela curva $D$, entre os pontos que a órbita de $X$ por qo cruza a órbita $\mathrm{D}$ e as seções $\Sigma_{1}$ e $\Sigma_{2}$, as seções $\Sigma_{1}$ e $\Sigma_{2}$ entre $\mathrm{D}$ e a reta $\mathrm{y}=\mathrm{n}_{5}$, e a reta $\mathrm{y}=\mathrm{n}_{5}$ entre $\Sigma_{1}$ e $\Sigma_{2}$. Se $\varepsilon_{0}>0$ for tomado de maneira que $\varepsilon_{0} \leq \mathrm{n}_{6},-\varepsilon \geq \mathrm{n}_{5}$ e as seções transversais $\Sigma_{3}$ e $\Sigma_{4}$ estão fora da faixa de regularização, então para $0<\varepsilon<\varepsilon_{0}$, o campo vetorial regularizado $Z_{\varepsilon}$ está entrando no anel B. 


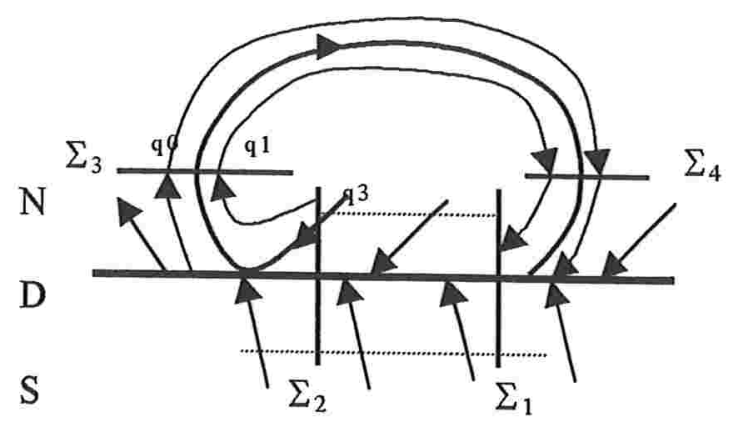

Fig. 49

Pela construção, no anel B não existem pontos singulares, logo, pelo Teorema de Poincaré-Bendixson, $Z_{\varepsilon}$ possui pelo menos uma órbita periódica em B. Será provado que toda órbita que existir em B será atratora e hiperbólica, portanto única.

Seja $\gamma_{\varepsilon}$ uma órbita periódica do campo vetorial regularizado $Z_{\varepsilon}$ em B, para $0<\varepsilon<\varepsilon_{0}$. É claro que as seções $\Sigma_{1}, \Sigma_{2}, \Sigma_{3}$ e $\Sigma_{4}$ são seções transversais à órbita $\gamma_{\varepsilon}$.

Seja $\eta_{\varepsilon}=\eta_{4 \varepsilon}{ }^{0} \eta_{3 \varepsilon}{ }^{0} \eta_{2 \varepsilon}{ }^{0} \eta_{1 \varepsilon}$ a aplicação de Poincaré da órbita $\gamma_{\varepsilon}$ definida na seção transversal $\Sigma_{1}$, onde cada $\eta_{\mathrm{i} \varepsilon}$ está definida entre $\Sigma_{\mathrm{i}}$ e $\Sigma_{\mathbf{i}+1}$, identificando-se $\Sigma_{5}=\Sigma_{1}$. A derivada da aplicação $\eta_{\varepsilon}$ será calculada usandose a derivada em cada trecho.

Sejam $\mathrm{p}_{1}=\left(\mathrm{x}_{1}, \mathrm{y}_{1}\right), \mathrm{p}_{2}=\left(\mathrm{x}_{2}, \mathrm{y}_{2}\right), \mathrm{p}_{3}=\left(\mathrm{x}_{3}, \mathrm{y}_{3}\right)$ e $\mathrm{p}_{4}=\left(\mathrm{x}_{4}, \mathrm{y}_{4}\right)$ os pontos onde a órbita $\gamma_{8}$ cruza as seções $\Sigma_{1}, \Sigma_{2}, \Sigma_{3}$ e $\Sigma_{4}$, respectivamente. Seja $\theta_{1}$, $\theta_{2}, \theta_{3}$ e $\theta_{4}$ os ângulos formados entre as seções transversais e a órbita $\gamma_{\varepsilon}$.

A seguir será estudada a aplicação de Poincaré entre cada trecho definido pelas seções transversais.

1) Entre $\Sigma_{1}$ e $\Sigma_{2}$;

Como primeira aproximação, neste trecho será considerado que os campos vetoriais $X$ e $Y$ são dados por $X(x, y)=(-1,-1), Y(x, y)=(-1,1)$.

Logo o campo regularizado é dado por

$$
\begin{aligned}
& Z_{\varepsilon}=\left(Z_{\varepsilon}^{1}, Z_{\varepsilon}^{2}\right)=\left(x^{\prime}, y^{\prime}\right)=\left(\left(1-\varphi_{\varepsilon}\right)(-1)+\varphi_{\varepsilon}(-1),\left(1-\varphi_{\varepsilon}\right) 1+\varphi_{\varepsilon}(-1)\right)= \\
& =\left(-1,1-2 \varphi_{\varepsilon}\right) .
\end{aligned}
$$

Este campo pode ser reduzido a 


$$
\begin{array}{cc} 
& \frac{\mathrm{dy}}{\mathrm{dx}}=\frac{1-2 \varphi_{\underline{\varepsilon}}}{-1} \\
\Rightarrow \quad & \frac{\mathrm{dy}}{1-2 \varphi_{\varepsilon}(\mathrm{y})}=\frac{\mathrm{dx}}{-1} \\
\Rightarrow \quad & \int_{\mathrm{y} 1}^{\mathrm{y} 1} \frac{1}{1-2 \varphi_{\varepsilon}(\mathrm{y})} d y=\int_{\mathrm{x} 1}^{\mathrm{x} 2}-\mathrm{dx}=\mathrm{x}_{1}-\mathrm{x}_{2}
\end{array}
$$

$\mathrm{Na}$ equação integral acima, os valores de $\mathrm{x}_{1}$ e $\mathrm{x}_{2}$ correspondem às seções transversais $\Sigma_{1}$ e $\Sigma_{2}$, respectivamente. Dado o valor de $y_{1}$, resolvendo-se a equação encontra-se o valor de $y_{2}$ tais que a órbita do campo regularizado que passa por $\left(\mathrm{x}_{1}, \mathrm{y}_{1}\right) \in \Sigma_{1}$ cruza a seção $\Sigma$ em $\left(\mathrm{x}_{2}, \mathrm{y}_{2}\right)$. As figuras abaixo exemplificam esta situação.

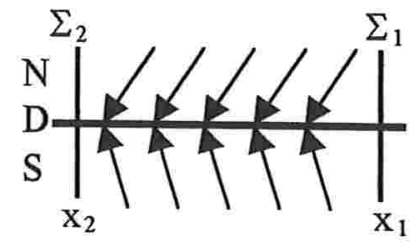

Fig. 50

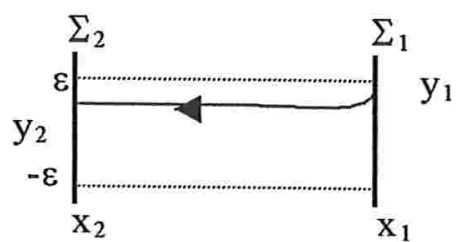

Fig. 51

Para entender como a variável y se comporta pode-se utilizar o gráfico abaixo, onde o eixo horizontal representa a variável y e o eixo vertical representa a função $\psi(y)=$

$$
\overline{1-2 \varphi_{\varepsilon}(y)}
$$

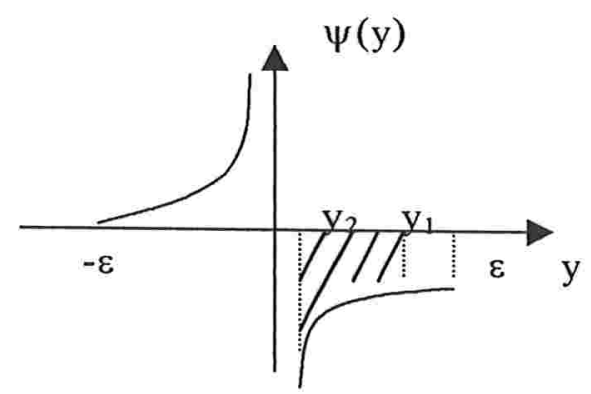

Fig. 52

Observa-se que o gráfico da figura 52 foi feito considerando-se que $\varphi(0)=1 / 2$, ou, equivalentemente, que $0=\varphi^{-1}(1 / 2)$. Caso isto não seja verdade, a assíntota não será o eixo vertical, mas a reta $y=\varphi^{-1}(1 / 2)$, que deverá estar próxima do eixo vertical. 
Interpretando-se a equação integral (2), com o auxílio do gráfico, tem-se que, fixado os valores de $\varepsilon$ e de $y_{1}$, o valor $y_{2}$ é aquele tal que a área hachurada na figura 52 vale $\left(\mathrm{x}_{1}-\mathrm{x}_{2}\right)$.

Portanto, quanto menor for $\varepsilon$, mais próximo da origem estará $\mathrm{y}_{2}$, podendo o valor de $\mathrm{y}_{1}$ ter sido tomado positivo ou negativo. integral,

Para estabelecer a dependência entre $\mathrm{y}_{2} \mathrm{e} \mathrm{y}_{1}$, retoma-se a equação

$$
\begin{aligned}
& \Psi\left(\mathrm{y}_{1}\right)=\int_{\mathrm{y} 1}^{\mathrm{y}^{2}} \frac{1}{1-2 \varphi_{\varepsilon}(\mathrm{y})} \mathrm{dy}=\left(\mathrm{x}_{1}-\mathrm{x}_{2}\right)=\mathrm{H}\left(\mathrm{y}_{2}\right)-\mathrm{H}\left(\mathrm{y}_{1}\right) \\
& \frac{\partial \Psi\left(y_{1}\right)}{\partial y_{1}}=H^{\prime}\left(y_{2}\right) \frac{d y_{2}}{d y_{1}}-H^{\prime}\left(y_{1}\right)=\frac{1}{1-2 \varphi_{\varepsilon}\left(y_{2}\right)} \frac{d y_{2}}{d y_{1}}-\frac{1}{1-2 \varphi_{\varepsilon}\left(y_{1}\right)}=0 \\
& \Rightarrow \quad \frac{\mathrm{dy}_{\underline{2}}}{\mathrm{dy}_{1}}=\frac{1}{1-2 \varphi_{\mathrm{s}}\left(\mathrm{y}_{1}\right)} \cdot\left(1-2 \varphi_{\varepsilon}\left(\mathrm{y}_{2}\right)\right) \\
& \Rightarrow \quad 0<\frac{\mathrm{dy}_{\underline{2}}}{\mathrm{dy}_{1}}=\frac{1-2 \varphi_{\underline{\varepsilon}}\left(\mathrm{y}_{\underline{2}}\right)}{1-2 \varphi_{\varepsilon}\left(\mathrm{y}_{1}\right)}<<1 .
\end{aligned}
$$

Afirma-se que $\lim _{\varepsilon \rightarrow 0} \mathrm{y}_{2}=\varphi^{-1}(1 / 2)$.

De fato, esta afirmação decorre dos cálculos e gráficos acima.

De (1) tem-se que

$$
\frac{\mathrm{dy}}{\mathrm{dt}}=1-2 \varphi_{\varepsilon} \quad \Rightarrow \quad \frac{\mathrm{dt}}{\mathrm{dy}}=\frac{1}{1-2 \varphi_{\varepsilon}} .
$$
dada por

A derivada da aplicação de Poincaré $\eta_{1 \varepsilon}$ da órbita $\gamma_{\varepsilon}$ entre $\Sigma_{1}$ e $\Sigma_{2}$ é

$$
\eta_{1 \varepsilon}{ }^{\prime}\left(p_{1}\right)=\frac{\cos \theta_{1}}{\cos \theta_{2}} \frac{Z_{\varepsilon}\left(\underline{p}_{1}\right) \mid}{Z_{\varepsilon}\left(p_{2}\right) \mid} \exp \left[\int_{\mathrm{t} 1}^{\mathrm{t} 2} \operatorname{div} Z_{\varepsilon}\left(\gamma_{\varepsilon}(\mathrm{t})\right) \mathrm{dt}\right]=
$$

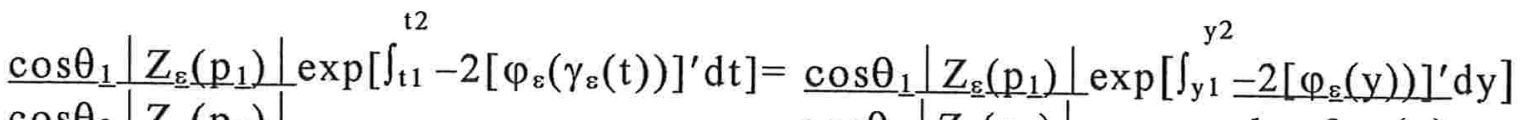

$$
\begin{aligned}
& \cos \theta_{2}\left|Z_{\varepsilon}\left(p_{2}\right)\right| \quad \frac{\cos \theta_{2}\left|Z_{\varepsilon}\left(p_{2}\right)\right|}{1-2 \varphi_{\varepsilon}(y)} \\
& =\frac{\cos \theta_{1}\left|\frac{Z_{\varepsilon}}{\left(p_{1}\right)}\right| \exp \left[\ln \left|1-2 \varphi_{\varepsilon}\left(\mathrm{y}_{2}\right)\right|-\ln \left|1-2 \varphi_{\varepsilon}\left(\mathrm{y}_{1}\right)\right|\right]}{\cos \theta_{2}\left|Z_{\varepsilon}\left(p_{2}\right)\right|} \\
& =\frac{\cos \theta_{1}}{\cos \theta_{2}} \frac{\left|Z_{\varepsilon}\left(p_{1}\right)\right|}{\left|Z_{\varepsilon}\left(p_{2}\right)\right|} \frac{\left|1-2 \varphi_{\varepsilon}\left(\mathrm{y}_{2}\right)\right|}{\left|1-2 \varphi_{\varepsilon}\left(\mathrm{y}_{1}\right)\right|}
\end{aligned}
$$


2) Entre $\Sigma_{2}$ e $\Sigma_{3}$

Neste trecho serão consideradas coordenadas locais tais que os campos X e Y são dados como abaixo

$$
X(x, y)=(-1,-x), Y(x, y)=(-1,1)
$$

Nestas coordenadas, o ponto D-dobra é o ponto $\mathrm{p}=(0,0)$.

O campo regularizado é dado por

$$
Z_{\varepsilon}=\left(Z_{\varepsilon}^{1}, Z_{\varepsilon}^{2}\right)=\left(x^{\prime}, y^{\prime}\right)=\left(-1,\left(1-\varphi_{\varepsilon}\right)-x \varphi_{\varepsilon}\right)
$$

Tem-se

$$
\frac{\mathrm{dy}}{\mathrm{dt}}=1-\varphi_{\varepsilon}-\mathrm{x} \varphi_{\varepsilon} \Rightarrow \quad \frac{\mathrm{dt}}{\mathrm{dy}}=\frac{1}{1-\varphi_{\varepsilon}-\mathrm{x} \varphi_{\varepsilon}}
$$

Logo, a derivada da aplicação de Poincaré $\eta_{2 \varepsilon}$ da órbita $\gamma_{8}$ entre $\Sigma_{2}$ e $\Sigma_{3}$ é dada por

$$
\begin{aligned}
& \eta_{2 \varepsilon}{ }^{\prime}\left(\mathrm{p}_{2}\right)=\frac{\cos \theta_{2}}{\cos \theta_{3}} \frac{\mathrm{Z}_{\varepsilon}}{\left|\mathrm{Z}_{\varepsilon}\left(\mathrm{p}_{2}\right)\right|} \exp \left[\int_{\mathrm{t} 2}^{\mathrm{t} 2} \operatorname{div} \mathrm{Z}_{\varepsilon}\left(\gamma_{\varepsilon}(\mathrm{t})\right) \mathrm{dt}\right]= \\
& =\frac{\cos \theta_{2}}{\cos \theta_{3}} \frac{\mid Z_{\varepsilon}}{\left|Z_{\varepsilon}\left(p_{2}\right)\right|} \mid \exp \left[\int_{t 2}^{t 3}\left[\varphi_{\varepsilon}\left(\gamma_{\varepsilon}(t)\right)\right]^{\prime}(-1-x) d t\right]= \\
& =\frac{\cos \theta_{2}}{\cos \theta_{3}} \frac{\left|Z_{\varepsilon}\left(\underline{p}_{2}\right)\right|}{\left|Z_{\varepsilon}\left(p_{3}\right)\right|} \exp \left[\int_{\mathrm{y} 2}^{\mathrm{y} 3} \frac{\left[\varphi_{\varepsilon}(y)\right]^{\prime}(-1-\mathrm{x})}{\varphi_{\varepsilon}(\mathrm{y})(-1-\mathrm{x})+1} d \mathrm{y}\right] \\
& =\frac{\cos \theta_{2}}{\cos \theta_{3}} \frac{\left|Z_{\varepsilon}\left(p_{2}\right)\right|}{\left|Z_{\varepsilon}\left(p_{3}\right)\right|} \exp \left[\ln \left|1-\varphi_{\varepsilon}\left(\mathrm{y}_{3}\right)\left(1+\mathrm{x}_{3}\right)\right|-\ln \left|1-\varphi_{\varepsilon}\left(\mathrm{y}_{2}\right)\left(1+\mathrm{x}_{2}\right)\right|\right] \\
& =\frac{\cos \theta_{2}}{\cos \theta_{3}} \frac{\left|Z_{\varepsilon}\left(p_{2}\right)\right|}{\left|Z_{\varepsilon}\left(p_{3}\right)\right|} \frac{\left|1-\varphi_{\varepsilon}\left(y_{3}\right)\left(1+x_{3}\right)\right|}{\left|1-\varphi_{\varepsilon}\left(y_{2}\right)\left(1+x_{2}\right)\right|} .
\end{aligned}
$$

3) Entre $\Sigma_{3}$ e $\Sigma_{4}$;

Neste trecho, para $0<\varepsilon<\varepsilon_{0}$, a órbita $\gamma_{\varepsilon}$ está fora da faixa de regularização, portanto a órbita só possui pontos de X. Ainda que a semiórbita de $X$ que está sendo utilizada possa variar com $\varepsilon$, a derivada da aplicação de Poincaré pode ser considerada limitada pelo máximo que ela atinge. Logo, tem-se 


$$
\eta_{3 \varepsilon^{\prime}}\left(\mathrm{p}_{3}\right)=\frac{\cos \theta_{3}}{\cos \theta_{4}\left|Z_{\varepsilon}\left(\mathrm{z}_{4}\right)\right|}\left(\mathrm{z}_{\underline{3}}\right) \mid K \text {, onde } K \in \mathfrak{R} \text { é uma constante. }
$$

4) Entre $\Sigma_{4}$ e $\Sigma_{1}$

Neste trecho serão consideradas as mesmas coordenadas utilizadas entre $\Sigma_{1}$ e $\Sigma_{2}$.

Logo,

$$
\eta_{4 \varepsilon}^{\prime}\left(p_{4}\right)=\frac{\cos \theta_{4}}{\cos \theta_{1}} \frac{\left|z_{\varepsilon}\left(p_{4}\right)\right|}{\left|Z_{\varepsilon}\left(p_{1}\right)\right|} \frac{\left|1-2 \varphi_{\varepsilon}\left(y_{1}\right)\right|}{\left|1-2 \varphi_{\varepsilon}\left(y_{4}\right)\right|} \text {. }
$$

Compondo as derivadas em cada trecho tem-se

$$
\begin{aligned}
& \eta_{\varepsilon}{ }^{\prime}\left(\mathrm{p}_{1}\right)=\eta_{4 \varepsilon^{\prime}}\left(\mathrm{p}_{4}\right) \cdot \eta_{3 \varepsilon}{ }^{\prime}\left(\mathrm{p}_{3}\right) \cdot \eta_{2 \varepsilon}{ }^{\prime}\left(\mathrm{p}_{2}\right) \eta_{1 \varepsilon}{ }^{\prime}\left(\mathrm{p}_{1}\right)=
\end{aligned}
$$

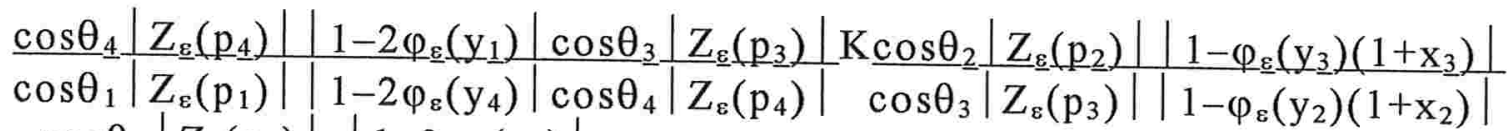

$$
\begin{aligned}
& \cdot \frac{\cos \theta_{1}}{\cos \theta_{2}} \frac{\left|Z_{\varepsilon}\left(p_{1}\right)\right|}{\left|Z_{\varepsilon}\left(p_{2}\right)\right|} \frac{\left|1-2 \varphi_{\varepsilon}\left(y_{2}\right)\right|}{\left|1-2 \varphi_{\varepsilon}\left(y_{1}\right)\right|}= \\
& =K \frac{\left|1-\varphi_{\varepsilon}\left(y_{3}\right)\left(1+x_{3}\right)\right|\left|1-2 \varphi_{\varepsilon}\left(y_{2}\right)\right|}{\left|1-2 \varphi_{\varepsilon}\left(y_{4}\right)\right|\left|1-\varphi_{\varepsilon}\left(y_{2}\right)\left(1+x_{2}\right)\right|}
\end{aligned}
$$$$
\text { Como } \varphi_{\varepsilon}\left(\mathrm{y}_{3}\right)=\varphi_{\varepsilon}\left(\mathrm{y}_{4}\right)=1 \text {, tem-se }
$$

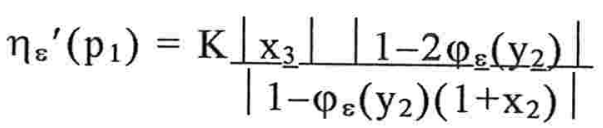

Por (3) e do fato que nas coordenadas utilizadas tem-se $x_{2}<0$, segue que, diminuindo-se $\varepsilon_{0}$ se necessário,

$$
\eta_{\varepsilon}{ }^{\prime}\left(\mathrm{p}_{1}\right)<1 \text {, para } 0<\varepsilon<\varepsilon_{0}
$$

Logo, a órbita $\gamma_{\varepsilon}$ é uma órbita periódica hiperbólica e atratora do campo vetorial regularizado $Z_{\varepsilon}$.

Os cálculos acima foram feitos usando-se coordenadas simplificadas como aproximação do campo $Z$, porém a conclusão pode ser extendida para um campo vetorial no caso geral, porque o aspecto determinante é o dado pela expressão (3), que permite o controle da derivada da aplicação de Poincaré. Esta expressão continua válida para o caso geral. 


\section{Regularização de Pontos Pertencentes à Interseç̧ão da Curva $D$ com $\partial M$.}

Neste parágrafo é estudada a regularização de pontos na intersecção da curva D com $\partial \mathrm{M}$. O objetivo é estabelecer as condições para que o campo regularizado seja localmente transversal a $\partial \mathrm{M}$ ou que surja uma tangência quadrática. Este assunto não foi abordado no trabalho de Sotomayor e Teixeira [S-T].

\subsection{Proposição}

Sejam $Z=(X, Y) \in \Omega^{r}(M, f), r \geq 1, e p \in D \cap \partial M \operatorname{com} X \beta(p) Y \beta(p)<0$ e $X \beta(p) Y f(p)-X f(p) Y \beta(p) \neq 0$. Então

(i) Se $X \beta(p) Y f(p)-X f(p) Y \beta(p)>0$, dada uma função de transição $\varphi$, existe $\varepsilon_{0}>0$ tal que se $0<\varepsilon<\varepsilon_{0}$, o campo regularizado $Z_{\varepsilon}$ possui um ponto de tangência quadrática interna na intersecção da faixa de regularização com $\partial M$;

(ii) Se $X \beta(p) Y f(p)-X f(p) Y \beta(p)<0$, dada uma função de transição $\varphi$, existe $\varepsilon_{0}>0$ tal que se $0<\varepsilon<\varepsilon_{0}$, o campo regularizado $Z_{\varepsilon}$ possui um ponto de tangência quadrática externa na intersecção da faixa de regularização com $\partial M$.

demonstração

Utilizando-se a Notação 2.4 do Capítulo 1, tem-se

$\mathrm{D}=\{\mathrm{y}=0\}, f(\mathrm{x}, \mathrm{y})=\mathrm{y}, \partial \mathrm{M}=\{\mathrm{x}=0\}, \beta(\mathrm{x}, \mathrm{y})=\mathrm{x}, \mathrm{p}=(0,0), \mathrm{X}(\mathrm{x}, \mathrm{y})=$ $(\mathrm{a}(\mathrm{x}, \mathrm{y}), \mathrm{b}(\mathrm{x}, \mathrm{y}))$ e $\mathrm{Y}(\mathrm{x}, \mathrm{y})=(\mathrm{c}(\mathrm{x}, \mathrm{y}), \mathrm{d}(\mathrm{x}, \mathrm{y})) . \operatorname{Logo}, X \beta(\mathrm{x}, \mathrm{y})=\mathrm{a}(\mathrm{x}, \mathrm{y})$, $\mathrm{X} f(\mathrm{x}, \mathrm{y})=\mathrm{b}(\mathrm{x}, \mathrm{y}), \mathrm{Y} \beta(\mathrm{x}, \mathrm{y})=\mathrm{c}(\mathrm{x}, \mathrm{y})$ e $\mathrm{Y} f(\mathrm{x}, \mathrm{y})=\mathrm{d}(\mathrm{x}, \mathrm{y})$.

Como $X \beta(p) Y \beta(p)=a(0) c(0)<0$, segue que a(0) e c(0) são diferentes de zero e têm sinais contrários no ponto $p=(0,0)$. Pela continuidade das funções envolvidas, esta situação se mantém numa vizinhança $\vartheta$ de p. Pode-se então usar o Lema 1.3 e tomar $\varepsilon_{1}>0$ tal que existem constantes $0<\mathrm{h}_{0}<\mathrm{h}_{1}<1$ tais que $0<\mathrm{h}_{0} \leq \mathrm{h}(\mathrm{y}) \leq \mathrm{h}_{1}<1$, para $|\mathrm{y}|<\varepsilon_{1}$, onde $\mathrm{h}(\mathrm{y})=\mathrm{c}(0, \mathrm{y}) /(\mathrm{c}(0, \mathrm{y})-\mathrm{a}(0, \mathrm{y}))$.

Se necessário, diminui-se $\varepsilon_{1}$ para que $(0, y) \in \vartheta$ se $|y|<\varepsilon_{1}$. 


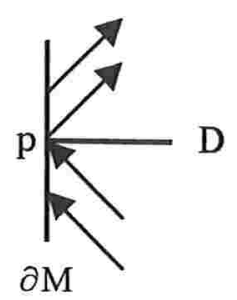

Fig. 53

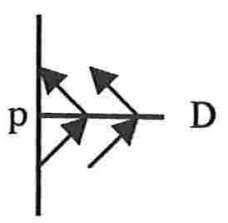

$\partial \mathrm{M}$

Fig. 55

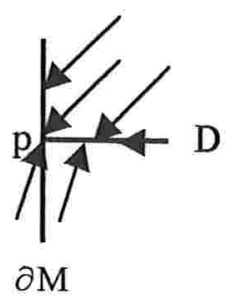

Fig. 57

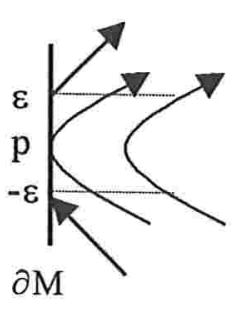

Fig. 54

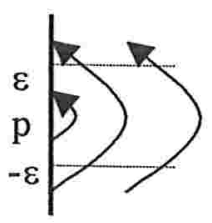

$\partial \mathrm{M}$

Fig. 56

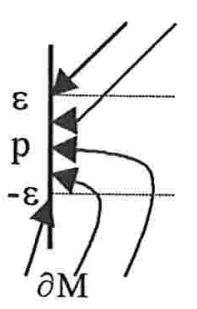

Fig. 58

As figuras 53 e 58 representam pontos p pertencentes à intersecção entre a fronteira de $M$ e a curva $D$ tais que $X \beta(p) Y \beta(p)<0$ e a sua regularização.

O campo regularizado é dado por $Z_{\varepsilon}=\left(\left(1-\varphi_{\varepsilon}\right) c+a \varphi_{\varepsilon},\left(1-\varphi_{\varepsilon}\right) d+b \varphi_{\varepsilon}\right)$. Portanto, para ter-se um ponto de tangência deve-se ter $Z_{\varepsilon} \beta(0, y)=\left(1-\varphi_{\varepsilon}(y)\right) c(0, y)+a(0, y) \varphi_{\varepsilon}(y)=0$.

Seja a função $g(y)=\left(1-\varphi_{\varepsilon}(y)\right) c(0, y)+a(0, y) \varphi_{\varepsilon}(y)$. Afirma-se que existe $\mathrm{y}_{\varepsilon} \in(-\varepsilon, \varepsilon)$ tal que $\mathrm{g}\left(\mathrm{y}_{\varepsilon}\right)=0$.

De fato,

$$
\begin{aligned}
& g(\varepsilon)=\left(1-\varphi_{\varepsilon}(\varepsilon)\right) c(0, \varepsilon)+a(0, \varepsilon) \varphi_{\varepsilon}(\varepsilon)=0 \cdot c(0, \varepsilon)+a(0, \varepsilon) \cdot 1=a(0, \varepsilon) \\
& g(-\varepsilon)=\left(1-\varphi_{\varepsilon}(-\varepsilon)\right) c(0,-\varepsilon)+a(0,-\varepsilon) \varphi_{\varepsilon}(-\varepsilon)=1 \cdot c(0,-\varepsilon)+a(0,-\varepsilon) \cdot 0=c(0,-\varepsilon) .
\end{aligned}
$$

Por hipótese, $a(0, \varepsilon)$ e $c(0,-\varepsilon)$ têm sinais contrários, logo, por continuidade, existe $\mathrm{y}_{\varepsilon} \in(-\varepsilon, \varepsilon)$ tal que $\mathrm{g}\left(\mathrm{y}_{\varepsilon}\right)=0$, e portanto o campo regularizado $Z_{\varepsilon}$ tangencia $\partial \mathrm{M}$ em $\left(0, \mathrm{y}_{\varepsilon}\right)$. 
Agora resta mostrar que esta tangência é quadrática, única e verificar quando é interna e quando é externa. Na verdade, não é necessário provar-se a unicidade, pois ela decorre diretamente do fato de que toda tangência que existir será sempre interna ou sempre externa (dependendo do sinal de $a(0) d(0)-b(0) c(0)$ ).

$$
\begin{gathered}
\nabla Z_{\varepsilon} \beta=\left(c_{x}\left(1-\varphi_{\varepsilon}\right)+a_{x} \varphi_{\varepsilon},-\varphi_{\varepsilon}^{\prime} c+c_{y}\left(1-\varphi_{\varepsilon}\right)+\varphi^{\prime}{ }_{\varepsilon} a+a_{y} \varphi_{\varepsilon}\right) . \\
Z^{2} \beta=\left(\left(1-\varphi_{\varepsilon}\right) c+\varphi_{\varepsilon} a,\left(1-\varphi_{\varepsilon}\right) d+\varphi_{\varepsilon} b\right) \cdot\left(c_{x}\left(1-\varphi_{\varepsilon}\right)+a_{x} \varphi_{\varepsilon},-\varphi^{\prime}{ }_{\varepsilon} c+\right. \\
\left.c_{y}\left(1-\varphi_{\varepsilon}\right)+\varphi^{\prime}{ }_{\varepsilon} a+a_{y} \varphi_{\varepsilon}\right)= \\
=\left(1-\varphi_{\varepsilon}\right)^{2} c_{x}+a_{x} c \varphi_{\varepsilon}\left(1-\varphi_{\varepsilon}\right)+a c_{x} \varphi_{\varepsilon}\left(1-\varphi_{\varepsilon}\right)+a a_{x}\left(\varphi_{\varepsilon}\right)^{2}-c d \varphi^{\prime}{ }_{\varepsilon}\left(1-\varphi_{\varepsilon}\right)+ \\
c_{y} d\left(1-\varphi_{\varepsilon}\right)^{2}+a d \varphi^{\prime}{ }_{\varepsilon}\left(1-\varphi_{\varepsilon}\right)+a_{y} d \varphi_{\varepsilon}\left(1-\varphi_{\varepsilon}\right)-b c \varphi^{\prime}{ }_{\varepsilon} \varphi_{\varepsilon}+b c_{y} \varphi_{\varepsilon}\left(1-\varphi_{\varepsilon}\right)+a b \varphi^{\prime}{ }_{\varepsilon} \varphi_{\varepsilon}+ \\
a_{y} b\left(\varphi_{\varepsilon}\right)^{2}
\end{gathered}
$$

No ponto $\left(0, \mathrm{y}_{\mathrm{\varepsilon}}\right)$, tem-se

$$
\varphi_{\varepsilon}\left(\mathrm{y}_{\varepsilon}\right)=\mathrm{c}\left(0, \mathrm{y}_{\varepsilon}\right) /\left(\mathrm{c}\left(0, \mathrm{y}_{\varepsilon}\right)-\mathrm{a}\left(0, \mathrm{y}_{\varepsilon}\right)\right)=\mathrm{h}\left(\mathrm{y}_{\varepsilon}\right) .
$$

Logo, no ponto $\left(0, y_{8}\right)$,

$$
\begin{aligned}
Z^{2} \beta= & \left(a^{2} c c_{x}-a a_{x} c^{2}-a^{2} c_{x}+a a_{x} c^{2}+\varphi^{\prime}{ }_{\varepsilon} a c d(c-a)+a^{2} c_{y} d-\right. \\
\varphi^{\prime}{ }_{\varepsilon} a^{2} d(c-a)- & \left.a a_{y} c d-\varphi^{\prime}{ }_{\varepsilon} b c^{2}(c-a)-a b c c_{y}+\varphi^{\prime}{ }_{\varepsilon} a b c(c-a)+a b b c^{2}\right) /(c-a)^{2}= \\
= & \left(a c^{2} d \varphi^{\prime}{ }_{\varepsilon}-a^{2} c d \varphi^{\prime}{ }_{\varepsilon}+a^{2} c_{y} d-a^{2} c d \varphi^{\prime}{ }_{\varepsilon}+a^{3} d \varphi^{\prime}{ }_{\varepsilon}-a a_{y} c d-b c^{3} \varphi^{\prime}{ }_{\varepsilon}+\right. \\
& \left.+a b c^{2} \varphi^{\prime}{ }_{\varepsilon}-a b c c_{y}+a b c^{2} \varphi^{\prime}{ }_{\varepsilon}-a^{2} b c \varphi^{\prime}{ }_{\varepsilon}+a_{y} b c^{2}\right) /(c-a)^{2}= \\
= & \left(a d \varphi^{\prime}{ }_{\varepsilon}\left(c^{2}-2 a c+a^{2}\right)-b c \varphi^{\prime}{ }_{\varepsilon}\left(c^{2}-2 a c+a^{2}\right)+a d\left(a c_{y}-a_{y} c\right)-\right. \\
& \left.-b c\left(a c_{y}-a_{y} c\right)\right) /(c-a)^{2}= \\
= & \left((a d-b c)\left(\varphi^{\prime}{ }_{\varepsilon}(c-a)^{2}+a c_{y}-a_{y} c\right)\right) /(c-a)^{2}
\end{aligned}
$$

Para estudar-se o sinal de $Z_{\varepsilon}^{2} \beta\left(0, y_{\varepsilon}\right)$ basta estudar-se o sinal de

(I) $\left(\varphi^{\prime}{ }_{\varepsilon}\left(\mathrm{y}_{\varepsilon}\right)\left(\mathrm{c}\left(0, \mathrm{y}_{\varepsilon}\right)-\mathrm{a}\left(0, \mathrm{y}_{\varepsilon}\right)\right)^{2}+\mathrm{a}\left(0, \mathrm{y}_{\varepsilon}\right) \mathrm{c}_{\mathrm{y}}\left(0, \mathrm{y}_{\varepsilon}\right)-\mathrm{a}_{\mathrm{y}}\left(0, \mathrm{y}_{\varepsilon}\right) \mathrm{c}\left(0, \mathrm{y}_{\varepsilon}\right)\right)$,

porque $\left(c\left(0, y_{\varepsilon}\right)-a\left(0, y_{\varepsilon}\right)\right)^{2}$ é estritamente positivo e $a\left(0, y_{\varepsilon}\right) d\left(0, y_{\varepsilon}\right)$ $\mathrm{b}\left(0, \mathrm{y}_{\mathrm{\varepsilon}}\right) \mathrm{c}\left(0, \mathrm{y}_{\mathrm{\varepsilon}}\right)$ tem o mesmo sinal que $\mathrm{a}(0) \mathrm{d}(0)-\mathrm{b}(0) \mathrm{c}(0)$.

Afirma-se que podemos tomar $0<\varepsilon_{0} \leq \varepsilon_{1}$ tal que se $0<\varepsilon \leq \varepsilon_{0}$, a expressão (I) é estritamente positiva.

De fato, basta usar-se o Lema $1.4 \mathrm{com}$ 


$$
\mathrm{K}>\sup \left\{\left(\mathrm{a}(0, \mathrm{y}) \mathrm{c}_{\mathrm{y}}(0, \mathrm{y})-\mathrm{a}_{\mathrm{y}}(0, \mathrm{y}) \mathrm{c}(0, \mathrm{y})\right) /(\mathrm{c}(0, \mathrm{y})-\mathrm{a}(0, \mathrm{y}))^{2},|\mathrm{y}| \leq \varepsilon_{1}\right\} \text {, }
$$

e tomar-se $\varepsilon_{0}=\min \left\{\varepsilon_{1}, \varepsilon_{2}\right\}$, onde $\varepsilon_{2}>0$ é dado pelo Lema 1.4 .

Pode-se concluir que então que se $0<\varepsilon \leq \varepsilon_{0}$, o campo regularizado $Z_{\varepsilon}$ possui um ponto de tangência quadrática $\left(0, \mathrm{y}_{\varepsilon}\right)$ com $\partial \mathrm{M}$, e esta tangência será interna se $\mathrm{a}(0) \mathrm{d}(0)-\mathrm{b}(0) \mathrm{c}(0)>0$, e externa caso $a(0) d(0)-b(0) c(0)<0$, pois este é o sinal de $Z^{2}{ }_{\varepsilon} \beta\left(0, y_{\varepsilon}\right)$.

\subsection{Proposição}

$\operatorname{Sejam} Z=(X, Y) \in \Omega^{r}(M, f), r \geq 1, e p \in D \cap \partial M \operatorname{com} X \beta(p) Y \beta(p)>0$. Então, dada uma função de transição $\varphi$, existe $\varepsilon_{0}>0$ tal que se $0<\varepsilon<\varepsilon_{0}$, o campo regularizado $Z_{\varepsilon}$ é transversal a $\partial M$ numa vizinhança de $p$.

\section{demonstração}

Utilizando-se a Notação 2.4 do Capítulo 1, tem-se

$\mathrm{D}=\{\mathrm{y}=0\}, f(\mathrm{x}, \mathrm{y})=\mathrm{y}, \partial \mathrm{M}=\{\mathrm{x}=0\}, \beta(\mathrm{x}, \mathrm{y})=\mathrm{x}, \mathrm{p}=(0,0), \mathrm{X}(\mathrm{x}, \mathrm{y})=$ $(\mathrm{a}(\mathrm{x}, \mathrm{y}), \mathrm{b}(\mathrm{x}, \mathrm{y}))$ e $\mathrm{Y}(\mathrm{x}, \mathrm{y})=(\mathrm{c}(\mathrm{x}, \mathrm{y}), \mathrm{d}(\mathrm{x}, \mathrm{y})) \cdot \log 0, X \beta(\mathrm{x}, \mathrm{y})=\mathrm{a}(\mathrm{x}, \mathrm{y}) \mathrm{e}$ $\mathrm{Y} \beta(\mathrm{x}, \mathrm{y})=\mathrm{c}(\mathrm{x}, \mathrm{y})$.

A condição $X \beta(p) Y \beta(p)>0$ implica no fato de as órbitas dos campos $\mathrm{X}$ e $\mathrm{Y}$ por $\mathrm{p}$ estarem ambas entrando em $\mathrm{M}$ ou ambas saindo. Em outras palavras, $X \beta(p)=a(0,0)$ e $Y \beta(p)=c(0,0)$ têm o mesmo sinal. Devido à continuidade destas funções, esta situação se mantém numa vizinhança $\vartheta$ do ponto $\mathrm{p}$.

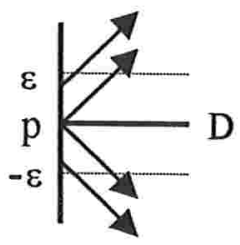

$\partial \mathrm{M}$

Fig. 59

A figura acima representa um ponto p pertencente à intersecção entre a fronteira de $M$ e a curva $D$ tal que $X \beta(p) Y \beta(p)>0$.

Toma-se $\varepsilon_{0}>0$ tal que se $0<\varepsilon<\varepsilon_{0}$, nas proximidades de $p$, a intersecção da faixa de regularização com $\partial \mathrm{M}$ está contida em $\vartheta$.

Logo, como $\left(1-\varphi_{\varepsilon}\right) \geq 0$ e $\varphi_{\varepsilon} \geq 0$, 


$$
Z_{\varepsilon} \beta(q)=\left(1-\varphi_{\varepsilon}(f(q))\right) Y \beta(q)+\varphi_{\varepsilon}(f(q)) X \beta(q)
$$

tem o mesmo sinal que $X \beta(q)$ e $Y \beta(q)$ se $q \in \vartheta$. Em particular, $Z_{\varepsilon} \beta$ não se anula e $Z_{\varepsilon}$ é transversal a $\partial \mathrm{M}$ em $\vartheta$.

\section{Campos Vetoriais Descontínuos com Regularização de Andronov- Pontrjaguin-Peixoto.}

Serão definidos a seguir conjuntos $G^{\mathrm{r}}(\mathrm{i}), \mathrm{i}=1, \ldots, 4$, auxiliares para as demonstração seguintes.

\subsection{Definição}

Denota-se por $\mathrm{G}^{\mathrm{r}}(\mathrm{i}), \mathrm{i}=1, \ldots, 4$, os subconjuntos de $\Omega^{\mathrm{r}}(\mathrm{M}, f)$ definidos abaixo

$\mathrm{G}^{\mathrm{r}}(1)=\left\{\mathrm{Z}=(\mathrm{X}, \mathrm{Y}) \in \Omega^{\mathrm{r}}(\mathrm{M}, f)|\mathrm{X}|_{\mathrm{N}}\right.$ e $\left.\mathrm{Y}\right|_{\mathrm{S}} \in \sum^{\mathrm{r}}$; todas as $\mathrm{D}$ singularidades de $Z$ são elementares; nenhuma singularidade de $F_{Z}$ pertence a $\partial \mathrm{M}\}$;

O conjunto $\mathrm{G}^{\mathrm{r}}(1)$ será responsável por todas as singularidades dos campos regularizados, para $\varepsilon$ pequeno, serem hiperbólicas.

$\mathrm{G}^{\mathrm{r}}(2)=\left\{\mathrm{Z}=(\mathrm{X}, \mathrm{Y}) \in \Omega^{\mathrm{r}}(\mathrm{M}, f)|\mathrm{X}|_{\mathrm{N}}\right.$ e $\left.\mathrm{Y}\right|_{\mathrm{S}} \in \Sigma^{\mathrm{r}}$; todas as poli-trajetórias fechadas de $Z$ são elementares e não possuem pontos de $\partial \mathrm{M}\}$;

O conjunto $\mathrm{G}^{\mathrm{r}}(2)$ será responsável por todas as órbitas periódicas dos campos regularizados, para $\varepsilon$ pequeno, serem hiperbólicas. Observa-se que será necessário exigir também que ocorra simultaneamente a situação descrita em $\mathrm{G}^{\mathrm{r}}(4)$, a seguir.

$\mathrm{G}^{\mathrm{r}}(3)=\left\{\mathrm{Z}=(\mathrm{X}, \mathrm{Y}) \in \Omega^{\mathrm{r}}(\mathrm{M}, f)|\mathrm{X}|_{\mathrm{N}}\right.$ e $\left.\mathrm{Y}\right|_{\mathrm{s}} \in \sum^{\mathrm{r}}$; se $\mathrm{p} \in \mathrm{D} \cap \partial \mathrm{M}$, então $\mathrm{X} \beta(\mathrm{p}) \mathrm{Y} \beta(\mathrm{p}) \neq 0$; caso $\mathrm{X} \beta(\mathrm{p}) \mathrm{Y} \beta(\mathrm{p})<0$, tem-se $\mathrm{X} \beta(\mathrm{p}) \mathrm{Y} f(\mathrm{p})-\mathrm{X} f(\mathrm{p}) \mathrm{Y} \beta(\mathrm{p}) \neq 0\}$;

O conjunto $G^{r}(3)$ será responsável pelas tangências dos campos regularizados, para $\varepsilon$ pequeno, serem no máximo quadráticas. A condição "se $\mathrm{p} \in \mathrm{D} \cap \partial \mathrm{M}$, então $\mathrm{X} \beta(\mathrm{p}) \mathrm{Y} \beta(\mathrm{p}) \neq 0$ " implicará que os campos $\mathrm{X}$ e $\mathrm{Y}$ não são tangentes ao bordo num ponto $\mathrm{p}$ pertencente a $\mathrm{D} \cap \partial \mathrm{M}$. $\mathrm{O}$ valor desta expressão ser positivo implicará que os campos regularizados serão transversais a $\partial \mathrm{M}$ numa vizinhança de $\mathrm{p}$. O valor desta expressão ser 
negativo significará que os campos regularizados terão uma tangência com $\partial \mathrm{M}$ numa vizinhança de $\mathrm{p}$. A condição "caso $\mathrm{X} \beta(\mathrm{p}) \mathrm{Y} \beta(\mathrm{p})<0$, tem-se $X \beta(p) Y f(p)-X f(p) Y \beta(p) \neq 0$ implicará que a tangência que surgiu na vizinhança de $\mathrm{p}$ é quadrática.

$\mathrm{G}^{\mathrm{r}}(4)=\left\{\mathrm{Z}=(\mathrm{X}, \mathrm{Y}) \in \Omega^{\mathrm{r}}(\mathrm{M}, f)|\mathrm{X}|_{\mathrm{N}}\right.$ e $\left.\mathrm{Y}\right|_{\mathrm{s}} \in \Sigma^{\mathrm{r}} ; \mathrm{Z}$ não possui conexão de separatrizes; se $p \in D \cap \partial M, X \beta(p) Y \beta(p)<0$ e $X \beta(p) Y f(p)-X f(p) Y \beta(p)>0$, então as órbitas e poli-trajetórias por $p$ não possuem separatrizes na sua composição e tampouco possuem um ponto $\mathrm{p}_{1} \neq \mathrm{p}, \mathrm{p}_{1} \in \mathrm{D} \cap \partial \mathrm{M}$ tal que $X \beta\left(p_{1}\right) Y \beta\left(p_{1}\right)<0$ e $\left.X \beta\left(p_{1}\right) Y f\left(p_{1}\right)-X f\left(p_{1}\right) Y \beta\left(p_{1}\right)>0\right\}$.

O conjunto $\mathrm{G}^{\mathrm{r}}(4)$ será responsável pela não existência de conexão de separatrizes dos campos vetoriais regularizados, para $\varepsilon$ pequeno. Observase que será necessário exigir-se também a ocorrência simultânea de $G^{r}(2)$ e $\mathrm{G}^{\mathrm{r}}(3)$.

\subsection{Definição}

Define-se $G^{r}(M)=G^{r}(1) \cap G^{r}(2) \cap G^{r}(3) \cap G^{r}(4)$.

\subsection{Proposição}

Seja $Z \in G^{r}(1), r \geq 1$. Então, dada uma função de transição $\varphi$, existe $\varepsilon_{0}>0$ tal que se $0<\varepsilon<\varepsilon_{0}, Z_{\varepsilon} \in \Sigma^{r}(1)$.

\section{demonstração}

Deve-se mostrar que se

$Z \in \mathrm{G}^{\mathrm{r}}(1)=\left\{\mathrm{Z} \in \Omega^{\mathrm{r}}(\mathrm{M}, f)|\mathrm{X}| \mathrm{N}\right.$ e $\mathrm{Y} \mid \mathrm{S} \in \Sigma^{\mathrm{r}}$, todas as Dsingularidades de $Z$ são elementares, nenhuma singularidade de $F_{Z}$ pertence a $\partial \mathrm{M}\}$,

então existe $\varepsilon_{0}>0$ tal que se $0<\varepsilon<\varepsilon_{0}$,

$Z_{\varepsilon} \in \Sigma^{\mathrm{r}}(1)=\left\{\mathrm{X} \in \mathcal{X}^{\mathrm{r}}(\mathrm{M}) \mid\right.$ todos os pontos singulares de $\mathrm{X}$ são hiperbólicos e estão contidos no interior de $\mathrm{M}\}$.

Como as D-singularidades de Z são elementares, elas são pontos singulares hiperbólicos de $\mathrm{F}_{Z}$ ou D-dobras.

Pela Proposição 1.5, se p é uma singularidade hiperbólica de $F_{Z}$, existe $\varepsilon_{0}>0$ tal que, para $0<\varepsilon<\varepsilon_{0}, Z_{\varepsilon}$ possui um único ponto crítico próximo a p, que é hiperbólico. 
Pela Proposição 1.1, se p é um ponto D-regular, existe $\varepsilon_{0}>0$ tal que, para $0<\varepsilon<\varepsilon_{0}, Z_{\varepsilon}$ não possui singularidades numa vizinhança de $p$.

Pela Proposição 1.6, em torno de uma D-dobra p existe $\varepsilon_{0}>0$ tal que, para $0<\varepsilon<\varepsilon_{0}, Z_{\varepsilon}$ não possui singularidades numa vizinhança de $p$.

Como $F_{Z}$ não possui singularidades em $\mathrm{D} \cap \partial \mathrm{M}$, tomando $\varepsilon_{0}>0$ pequeno, pela continuidade das funções envolvidas, pode-se garantir que, para $0<\varepsilon<\varepsilon_{0}, Z_{\varepsilon}$ não possui singularidades em $\partial \mathrm{M}$.

Resta mostrar que o valor de $\varepsilon_{0}$ pode ser tomado único e estritamente positivo.

De fato, seja $p \in D$. Então $p$ é um ponto D-regular, uma singularidade hiperbólica de $F_{Z}$ ou uma D-dobra. Para cada uma das três possibilidades, existe uma proposição que garante um valor $\varepsilon_{0}>0$ e uma vizinhança em torno de $p$ na qual todos os pontos de $Z_{\varepsilon}$, para $0<\varepsilon<\varepsilon_{0}$, são regulares ou a vizinhança possui uma singularidade hiperbólica isolada. A união destas vizinhanças cobre D, e pela compacidade de D (D é fechado e está contido em $M$, que é compacto, logo, pelo Teorema de Heine-Borel, $D$ é compacto), existe uma subcobertura formada por um número finito desta vizinhanças. Nesta subcobertura finita pode-se escolher $\varepsilon_{0}$ como o menor valor de $\varepsilon_{0}$ associado a estas vizinhanças. Este valor $\varepsilon_{0}$ é estritamente positivo porque é o ínfimo de um conjunto finito de valores positivos.

Por fim deve-se verificar se este valor de $\varepsilon_{0}>0$ é pequeno o suficiente para evitar que hajam pontos críticos na fronteira, e se não for ele ainda pode ser diminuído de modo a abranger também este caso.

Com isto a Proposição está demonstrada.

\subsection{Proposição}

Seja $Z \in G^{r}(2) \cap G^{r}(4), r \geq 1$. Então, dada uma função de transição $\varphi$, existe $\varepsilon_{0}>0$ tal que se $0<\varepsilon<\varepsilon_{0}, Z_{\varepsilon} \in \Sigma^{r}(2)$.

demonstração

Deve-se mostrar que se $Z \in G^{r}(2) \cap G^{r}(4)$, então existe $\varepsilon_{0}>0$ tal que se $0<\varepsilon<\varepsilon_{0}, Z_{\varepsilon} \in \Sigma^{r}(2)$.

Recorda-se que 
$\mathrm{G}^{\mathrm{r}}(2)=\left\{Z \in \Omega^{\mathrm{r}}(\mathrm{M}, f)|\mathrm{X}|_{\mathrm{N}}\right.$ e $\left.\mathrm{Y}\right|_{\mathrm{S}} \in \Sigma^{\mathrm{r}}$, todas as poli-trajetórias fechadas de $Z$ são elementares e não possuem pontos de $\partial \mathrm{M}\}$,

$\mathrm{G}^{\mathrm{r}}(4)=\left\{\mathrm{Z}=(\mathrm{X}, \mathrm{Y}) \in \Omega^{\mathrm{r}}(\mathrm{M}, f)|\mathrm{X}|_{\mathrm{N}}\right.$ e $\left.\mathrm{Y}\right|_{\mathrm{s}} \in \sum^{\mathrm{r}} ; \mathrm{Z}$ não possui conexão de separatrizes; se $p \in D \cap \partial M, X \beta(p) Y \beta(p)<0$ e $X \beta(p) Y f(p)$ $\mathrm{X} f(\mathrm{p}) \mathrm{Y} \beta(\mathrm{p})>0$, então as órbitas e poli-trajetórias por $\mathrm{p}$ não possuem separatrizes na sua composição e tampouco possuem um ponto $\mathrm{p}_{1} \neq \mathrm{p}, \mathrm{p}_{1} \in$ $D \cap \partial M$ tal que $X \beta\left(p_{1}\right) Y \beta\left(p_{1}\right)<0$ e $\left.X \beta\left(p_{1}\right) Y f\left(p_{1}\right)-X f\left(p_{1}\right) Y \beta\left(p_{1}\right)>0\right\}$.

e

$\Sigma^{\mathrm{r}}(2)=\left\{\mathrm{X} \in \mathcal{X}^{\mathrm{r}}(\mathrm{M}) \mid\right.$ todas as órbitas periódicas de $\mathrm{X}$ são hiperbólicas e estão contidas no interior de $\mathrm{M}\}$.

Como $\left.\mathrm{X}\right|_{\mathrm{N}}$ e $\left.\mathrm{Y}\right|_{\mathrm{S}} \in \Sigma^{\mathrm{r}}$, todas as órbitas periódicas de $\mathrm{X}$ e de $\mathrm{Y}$ são hiperbólicas, logo resta verificar o que ocorre com as órbitas periódicas que surgem com a regularização.

Seja $\gamma$ uma poli-trajetória fechada elementar de Z. Existem três possibilidades, $\gamma$ pode ser do tipo I, do tipo II ou do tipo III. As Proposições $1.7,1.8$ e 1.9 garantem que existe $\varepsilon_{0}>0$ pequeno tal que 0 campo regularizado $Z_{\varepsilon}$ possui uma órbita periódica hiperbólica próxima a $\gamma$, para $0<\varepsilon<\varepsilon_{0}$.

É claro que o valor de $\varepsilon_{0}$ pode ser tomado positivo e único pois do fato das poli-trajetórias serem elementares segue que são isoladas e portanto em número finito.

Como os campos X e Y são estruturalmente estáveis, todas as suas singularidades são hiperbólicas, não existindo a possibilidade de ocorrer uma bifurcação de Hopf que gere uma órbita periódica. As singularidades de $F_{Z}$ são do tipos nó ou sela e, mesmo que não sejam hiperbólicas, não podem gerar uma bifurcação de Hopf. Logo, está excluída a possibilidade do surgimento de órbitas periódicas, durante o processo de regularização, que tenham nascido de pontos singulares.

Do fato de $Z \in G^{\mathrm{r}}(4)$, em particular, tem-se que $Z$ não possui laços ou gráficos. Logo, também está excluída a possibilidade do surgimento de órbitas periódicas a partir deste tipo de situação.

Portanto, a regularização de poli-trajetórias fechadas é a única maneira de criar órbitas periódicas, ou seja, de criar órbitas periódicas que não fossem órbitas periódicas do campo $\mathrm{X}$ ou do campo $\mathrm{Y}$, e com isto conclui-se a proposição. 


\subsection{Proposição}

Seja $Z \in G^{r}(3)$. Então, dada uma função de transição $\varphi$, existe $\varepsilon_{0}>0$ tal que se $0<\varepsilon<\varepsilon_{0}, Z_{\varepsilon} \in \Sigma^{r}(3)$.

demonstração

Seja $Z \in G^{r}(3)$.

Recorda-se que

$\mathrm{G}^{\mathrm{r}}(3)=\left\{\mathrm{Z}=(\mathrm{X}, \mathrm{Y}) \in \Omega^{\mathrm{r}}(\mathrm{M}, f)|\mathrm{X}|_{\mathrm{N}}\right.$ e $\left.\mathrm{Y}\right|_{\mathrm{s}} \in \Sigma^{\mathrm{r}}$; se $\mathrm{p} \in \mathrm{D} \cap \partial \mathrm{M}$, então $\mathrm{X} \beta(\mathrm{p}) \mathrm{Y} \beta(\mathrm{p}) \neq 0$; caso $\mathrm{X} \beta(\mathrm{p}) \mathrm{Y} \beta(\mathrm{p})<0$, tem-se $\mathrm{X} \beta(\mathrm{p}) \mathrm{Y} f(\mathrm{p})-\mathrm{X} f(\mathrm{p}) \mathrm{Y} \beta(\mathrm{p}) \neq 0\}$; $\mathrm{e}$

$\Sigma^{\mathrm{r}}(3)=\left\{\mathrm{X} \in \mathcal{X}^{\mathrm{r}}(\mathrm{M}) \mid\right.$ para todo ponto $\mathrm{p} \in \partial \mathrm{M}$, a órbita de $\mathrm{X}$ por $\mathrm{p}, \varphi(\mathrm{t}, \mathrm{p})$, é transversal a $\partial \mathrm{M}$ (isto é, $(\partial / \partial \mathrm{t})(\beta \circ \varphi)(0, \mathrm{p}) \neq 0)$ ou tem contato parabólico com $\partial \mathrm{M}$ (isto é, $(\partial / \partial \mathrm{t})(\beta \circ \varphi)(0, \mathrm{p})=0$ e $\left.\left.\left(\partial^{2} / \partial \mathrm{t}^{2}\right)\left(\beta_{\circ} \varphi\right)(0, \mathrm{p}) \neq 0\right)\right\}$.

Basta que a proposição seja provada para o caso em que $\mathrm{D} \cap \partial \mathrm{M} \neq \phi$.

De fato, no caso em que $\mathrm{D} \cap \partial \mathrm{M}=\phi$, para $\varepsilon$ pequeno, a faixa de regularização não intercepta $\partial \mathrm{M}$, e portanto as tangências do campo $Z_{\varepsilon}$ com $\partial \mathrm{M}$ são as tangências de $\mathrm{X}$ e de $\mathrm{Y}$, que por hipótese são quadráticas.

Quando $\mathrm{D} \cap \partial \mathrm{M} \neq \phi$, torna-se necessário verificar quais tangências podem surgir em decorrência da regularização.

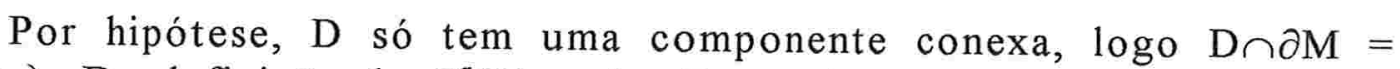
$\left\{\mathrm{p}_{1}, \mathrm{p}_{2}\right\}$. Da definição de $\mathrm{G}^{\mathrm{r}}(3)$ e das Proposições 2.1 e 2.2 , tem-se que existem $\varepsilon_{\mathrm{i}}>0, \mathrm{i}=1,2$, tais que se $0<\varepsilon<\varepsilon_{\mathrm{i}}$, na intersecção da faixa de regularização de $Z_{\varepsilon}$ com $\partial \mathrm{M}$ em torno de $p_{i}$, o campo $Z_{\varepsilon}$ possui uma tangência quadrática ou é transversal.

Toma-se $\varepsilon_{0}=\min \left\{\varepsilon_{1}, \varepsilon_{2}\right\}$ e a Proposição está demonstrada.

\subsection{Proposição}

Seja $Z \in G^{r}(4) \cap G^{r}(2) \cap G^{r}$ (3). Então, dada uma função de transição $\varphi$, existe $\varepsilon_{0}>0$ tal que se $0<\varepsilon<\varepsilon_{0}, Z_{\varepsilon} \in \Sigma^{r}(4)$.

demonstração

Seja $Z \in G^{\mathrm{r}}(4) \cap G^{\mathrm{r}}(2) \cap \mathrm{G}^{\mathrm{r}}(3)$. 
$\mathrm{G}^{\mathrm{r}}(4)=\left\{\mathrm{Z}=(\mathrm{X}, \mathrm{Y}) \in \Omega^{\mathrm{r}}(\mathrm{M}, f)|\mathrm{X}|_{\mathrm{N}}\right.$ e $\left.\mathrm{Y}\right|_{\mathrm{S}} \in \sum^{\mathrm{r}} ; \mathrm{Z}$ não possui conexão de separatrizes; se $p \in D \cap \partial M, X \beta(p) Y \beta(p)<0$ e $X \beta(p) Y f(p)-X f(p) Y \beta(p)>0$, então as órbitas e poli-trajetórias por $p$ não possuem separatrizes na sua composição e tampouco possuem um ponto $\mathrm{p}_{1} \neq \mathrm{p}, \mathrm{p}_{1} \in \mathrm{D} \cap \partial \mathrm{M}$ tal que $\mathrm{X} \beta\left(\mathrm{p}_{1}\right) \mathrm{Y} \beta\left(\mathrm{p}_{1}\right)<0$ e $\left.\mathrm{X} \beta\left(\mathrm{p}_{1}\right) \mathrm{Y} f\left(\mathrm{p}_{1}\right)-\mathrm{X} f\left(\mathrm{p}_{1}\right) \mathrm{Y} \beta\left(\mathrm{p}_{1}\right)>0\right\}$;

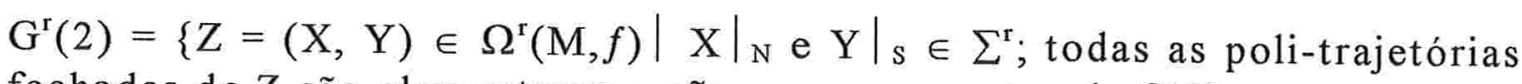
fechadas de $Z$ são elementares e não possuem pontos de $\partial \mathrm{M}\}$;

$\mathrm{G}^{\mathrm{r}}(3)=\left\{\mathrm{Z}=(\mathrm{X}, \mathrm{Y}) \in \Omega^{\mathrm{r}}(\mathrm{M}, f)|\mathrm{X}|_{\mathrm{N}}\right.$ e $\left.\mathrm{Y}\right|_{\mathrm{s}} \in \Sigma^{\mathrm{r}}$; se $\mathrm{p} \in \mathrm{D} \cap \partial \mathrm{M}$, então $\mathrm{X} \beta(\mathrm{p}) \mathrm{Y} \beta(\mathrm{p}) \neq 0$; caso $\mathrm{X} \beta(\mathrm{p}) \mathrm{Y} \beta(\mathrm{p})<0$, tem-se $\mathrm{X} \beta(\mathrm{p}) \mathrm{Y} f(\mathrm{p})-\mathrm{X} f(\mathrm{p}) \mathrm{Y} \beta(\mathrm{p}) \neq 0\}$; $\mathrm{e}$

$\Sigma^{\mathrm{r}}(4)=\left\{X \in \mathcal{K}^{\mathrm{r}}(\mathrm{M}) \mid\right.$ o campo vetorial $X$ não possui conexões de separatrizes de selas, de separatrizes de selas com separatrizes de tangência ou de separatrizes de tangências $\}$.

Primeiro será analisado o caso em que $\mathrm{D} \cap \partial \mathrm{M}=\phi$.

Neste caso, pode-se tomar $\varepsilon_{0}>0$ pequeno de modo que a faixa de regularização de $Z_{\varepsilon}$ não intercepte $\partial \mathrm{M}$ se $0<\varepsilon<\varepsilon_{0}$.

Afirma-se que, diminuindo-se $\varepsilon_{0}>0$ se necessário, para $0<\varepsilon<\varepsilon_{0}$, não há conexão de separatrizes para $Z_{\varepsilon}$.

De fato, como $\left.X\right|_{N}$ e $\left.Y\right|_{s} \in \Sigma^{r}$, e $Z$ não possui conexão de separatrizes, as possibilidades de surgirem conexões de separatrizes para $Z_{\varepsilon}$ seriam:

(i) através de pontos da curva de descontinuidade, como exemplificados nas figuras 56 a 58 ;

(ii) através do surgimento de uma tangência de ordem maior ou igual a três do campo regularizado com $\partial \mathrm{M}$, que poderia se desdobrar criando uma conexão de tangências;

(iii) através de uma órbita periódica semi-estável que poderia desaparecer, criando a conexão de uma sela interna à órbita com uma separatriz externa à órbita. 

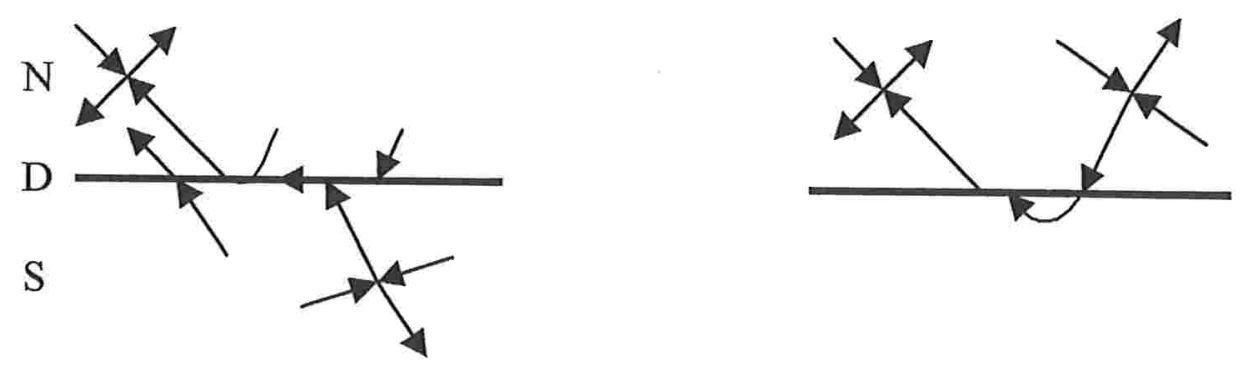

Fig. 60

Fig. 61

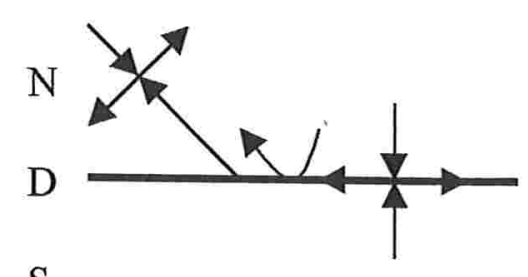

S

Fig. 62

A possibilidade (ii) é evitada com a exigência que $Z \in G^{\mathrm{r}}(3)$. De fato, neste caso no máximo surgirão tangências quadráticas do campo regularizado com $\partial \mathrm{M}$.

A possibilidade (iii) é evitada com a exigência que $Z \in G^{r}(2)$. De fato, neste caso todas as poli-trajetórias de $Z$ são elementares, e pela Proposição 3.4 (aqui também tem-se a hipótese que $Z \in G^{r}(4)$, exigida nesta Proposição), para $\varepsilon$ pequeno, os campos vetoriais regularizados possuem todas as suas órbitas periódicas hiperbólicas.

Resta analisar-se o caso (i):

Seja $\delta=\min \left\{\operatorname{dist}\left(\gamma_{\mathrm{i}}, \gamma_{\mathrm{j}}\right) \mid \gamma_{\mathrm{i}}\right.$ separatriz de $Z$ e $\left.\mathrm{i} \neq \mathrm{j}\right\}$. É claro que $\delta>0$, pois o número de separatrizes é finito.

Basta então que $\varepsilon_{0}>0$ seja diminuído, se necessário, de modo que a distância mínima entre as separatrizes não passe a ser menor que $\delta / 2$. Assim elas não poderão estar se unindo dentro da faixa de regularização.

No caso em que $\mathrm{D} \cap \partial \mathrm{M} \neq \phi$, basta analisar-se o que acontece quando surge um ponto de tangência quadrática de $Z_{\varepsilon}$ com $\partial \mathrm{M}$ na vizinhança de um ponto $\mathrm{p} \in \mathrm{D} \cap \partial \mathrm{M}$. De fato, as outras possíveis conexões de tangência já são evitadas como no caso $\mathrm{D} \cap \partial \mathrm{M}=\phi$.

Não ocorre uma conexão da tangência que surgiu em p com as outras separatrizes de $Z_{\varepsilon}$ porque as órbitas de $X$ e de $Y$ que passam por p não são 
separatrizes, e a última hipótese na definição de $\mathrm{G}^{\mathrm{r}}(4)$ evita a conexão de duas tangências internas no campo regularizado, que surgiram simultaneamente.

Observa-se que quando surge uma tangência interna a partir de um ponto $\mathrm{p} \in \mathrm{D} \cap \partial \mathrm{M} \operatorname{com} \mathrm{X} f(\mathrm{p}) \mathrm{Y} f(\mathrm{p})<0$, ou seja, p pertencente a um arco de $F_{Z}$, este mesmo arco não poderá ser separatriz de uma sela de $F_{Z}$ por incompatibilidade no sentido das órbitas. Por esse mesmo motivo este arco não poderá pertencer a uma outra tangência interna que esteja surgindo num ponto $\mathrm{p}_{1} \neq \mathrm{p}, \mathrm{p}_{1} \in \mathrm{D} \cap \partial \mathrm{M}$.

Logo, existe $\varepsilon_{0}>0$ de modo que o campo regularizado $Z_{\varepsilon}$ não possui conexão de separatrizes se $0<\varepsilon<\varepsilon_{0}$.

Será provado que o conjunto $\mathrm{G}^{\mathrm{r}}(\mathrm{M})$ é genérico. Para isto será aplicado o Teorema de Sard, enunciado a seguir.

\subsection{Teorema de Sard}

Sejam $M \subset \Re^{2}$ e $T: M \rightarrow \Re^{2}$ uma aplicação de classe $C^{1}$. Então o conjunto dos valores críticos de $T$, isto é, crit(T) $=\left\{v \in R^{2} / \exists p \in M\right.$ tal $q u e T(p)=v$ e $\operatorname{det}(D T(p))=0\}$ tem medida nula em $\Re^{2}$.

A demonstração deste teorema pode ser encontrada em [L2], entre outras referências.

\subsection{Teorema}

Se $Z \in G^{r}$, então, dada uma função de transição $\varphi$, existe $\varepsilon_{0}>0$ tal que se $0<\varepsilon<\varepsilon_{0}, Z_{\varepsilon} \in \Sigma^{r}$.

demonstração

Imediata das Proposições 3.3, 3.4, 3.5 e 3.6.

\subsection{Teorema}

$O$ conjunto $G^{r}(M)$ é aberto em $\Omega^{r}(M, f)$.

demonstração

Seja $Z \in G^{r}(M)$. 
Será provado que existe $\delta>0$ tal que se $Z^{*}=\left(\mathrm{X}^{*}, \mathrm{Y}^{*}\right) \in \Omega^{\mathrm{r}}(\mathrm{M}, f)$ e $\left|\mathrm{Z}-\mathrm{Z}^{*}\right|_{\mathrm{r}}=\max \left\{\left|\mathrm{X}-\mathrm{X}^{*}\right|_{\mathrm{r}},\left|\mathrm{Y}-\mathrm{Y}^{*}\right|_{\mathrm{r}}\right\}<\delta$, então $\mathrm{Z}^{*} \in \mathrm{G}^{\mathrm{r}}(\mathrm{M})$. Para isto será provado que $Z^{*}$ pertence a cada um dos conjuntos $\mathrm{G}^{\mathrm{r}}(\mathrm{i}), \mathrm{i}=1, \ldots, 4$.

- Afirma-se que existe $\delta_{1}>0$ tal que se $\left|Z-Z^{*}\right|<\delta_{1}$, então $Z^{*} \in G^{r}(1)$.

De fato, como $Z=(X, Y) \in G^{r}(M)$, tem-se $\left.X\right|_{N}$ e $\left.Y\right|_{S} \in \Sigma^{r}$, e da abertura do conjunto $\Sigma^{\mathrm{r}}$ (pelo Teorema de Peixoto, Cap. 1, Teorema 1.4), existe $\delta_{1}>0$ tal que se $\left|Z-Z^{*}\right|<\delta_{1}$, então $\left.X^{*}\right|_{N}$ e $\left.Y^{*}\right|_{s} \in \Sigma^{r}$.

Diminuindo-se $\delta_{1}>0$, se necessário, tem-se todas as Dsingularidades de $Z^{*}$ elementares e disjuntas de $\partial \mathrm{M}$. Prova-se esta observação mostrando-se que se p é uma $\mathrm{D}$-singularidade elementar de $\mathrm{Z}$ e $Z^{*}$ é um campo descontínuo próximo a $Z$, então existe um ponto $\mathrm{p}^{*}$, próximo a $\mathrm{p}$, que é uma $\mathrm{D}$-singularidade elementar de $Z^{*}$ do mesmo tipo que $p$.

De fato, se p é uma D-singularidade elementar, então p é uma Ddobra ou uma singularidade hiperbólica de $\mathrm{F}_{\mathrm{Z}}$.

Seja p uma D-dobra de Z. Pode-se supor, sem perda de generalidade, que $\mathrm{p}$ é um ponto de contato quadrático de $\mathrm{X}$, isto é, $\mathrm{X} f(\mathrm{p})=0$, $\mathrm{X}^{2} f(\mathrm{p}) \neq 0$ e $\mathrm{Y} f(\mathrm{p}) \neq 0$. Como $\mathrm{X} f(\mathrm{p})=0$ e $\mathrm{X}^{2} f(\mathrm{p}) \neq 0$, a curva $\{\mathrm{X} f=0\}$ cruza o eixo $x$ transversalmente no ponto $p$, e pela continuidade das funções envolvidas, diminuindo-se $\delta_{1}>0$ se necessário, o mesmo vale para $Z^{*}$ tal que $\left|Z-Z^{*}\right|<\delta_{1}$. Ou seja, existe $\mathrm{p}^{*} \in \mathrm{D}$ tal que $\mathrm{X}^{*} f\left(\mathrm{p}^{*}\right)=0$ e $\mathrm{X}^{* 2} f\left(\mathrm{p}^{*}\right) \neq 0$. Se $\delta_{1}>0$ é pequeno o suficiente pode-se garantir também que $\mathrm{Y}^{*} f\left(\mathrm{p}^{*}\right) \neq 0$. Portanto, conclue-se que $\mathrm{p}^{*}$ é uma $\mathrm{D}$-dobra de $\mathrm{Z}^{*}$.

Seja $\mathrm{p}$ uma singularidade hiperbólica de $\mathrm{F}_{\mathrm{Z}}$. Tem-se $\mathrm{X} f(\mathrm{p}) \mathrm{Y} f(\mathrm{p})<0$, $\operatorname{det}[X, Y](p)=0$ e $\operatorname{d}\left(\left.\operatorname{det}[X, Y]\right|_{D}\right)(p) \neq 0$. Analogamente ao caso da Ddobra, a curva $\{\operatorname{det}[X, Y]=0\}$ cruza transversalmente o eixo $x$ no ponto $p$, e o mesmo vale para $Z^{*} \delta_{1}$-próximo de $Z$. Portanto existe $p^{*} \in D$ tal que $\mathrm{X}^{*} f\left(\mathrm{p}^{*}\right) \mathrm{Y}^{*} f\left(\mathrm{p}^{*}\right)<0, \operatorname{det}\left[\mathrm{X}^{*}, \mathrm{Y}^{*}\right]\left(\mathrm{p}^{*}\right)=0$ e $\mathrm{d}\left(\left.\operatorname{det}\left[\mathrm{X}^{*}, \mathrm{Y}^{*}\right]\right|_{\mathrm{D}}\right)\left(\mathrm{p}^{*}\right) \neq 0$. Portanto $\mathrm{p}^{*}$ é um ponto crítico hiperbólico para $\mathrm{F}_{\mathrm{Z}^{*}}$. Como $\delta_{1}>0$ pode ser tomado de maneira que nenhuma das funções envolvidas tenha trocado de sinal, $\mathrm{p}^{*}$ é uma singularidade do mesmo tipo que $\mathrm{p}$.

É imediato que $\delta_{1}>0$ pode ser tomado de maneira que nenhuma das singularidades de $Z^{*}$ pertença a $\partial \mathrm{M}$ porque as singularidades de $\mathrm{Z}$ não pertencem a $\partial \mathrm{M}$ e as funções envolvidas são contínuas.

Pela compacidade de $\mathrm{D}$ e do fato das D-singularidades serem isoladas, $Z$ só possui um número finito de $\mathrm{D}$-singularidades, logo o valor $\delta_{1}$ pode ser tomado único e estritamente positivo. 
Pelo Corolário 1.2 e pela compacidade de $D$, segue que $Z^{*}$ não possui nenhuma outra singularidade além das descritas acima, isto é, daquelas que têm correspondência em singularidades de $\mathrm{Z}$.

$$
\text { Logo, } Z^{*} \in \mathrm{G}^{\mathrm{r}}(1) \text {. }
$$

- Afirma-se que existe $\delta_{2}>0$ tal que se $\left|Z-Z^{*}\right|<\delta_{2}$, então $Z^{*} \in G^{r}(2)$.

Sabe-se que, como $Z=(X, Y) \in G^{r}(2)$, então $\left.X\right|_{N}$ e $\left.Y\right|_{S} \in \sum^{r}$, e todas as poli-trajetórias fechadas de $Z$ são elementares e não possuem pontos de $\partial \mathrm{M}$. tem-se

Seja $\gamma$ uma poli-trajetória fechada elementar de Z. Pela definição,

(i) $\quad \gamma$ é do tipo I e $\eta^{\prime}(\mathrm{p}) \neq 1$, para algum $\mathrm{p} \in \gamma$; ou

(ii) $\gamma$ é do tipo II; ou

(iii) $\gamma$ é do tipo III e todos os arcos de trajetória de $F_{Z}$ são de deslizamentos ou todos são arcos de escape.

Suponha-se que $\gamma$ é uma poli-trajetória fechada elementar tipo I de $Z$. Associada à $\gamma$ existe uma aplicação de Poincaré $\eta$, diferenciável e tal que $\eta^{\prime}(p) \neq 1$, para algum ponto $p \in \gamma$. Ou seja, p é um ponto fixo hiperbólico do difeomorfismo $\eta$. Logo, existe $k>0$ tal que se $\mu$ é um difeomorfismo com $|\eta-\mu|<\mathrm{k}$, então $\mu$ possui um ponto fixo hiperbólico $\mathrm{p}_{\mu}$, próximo a p. Basta então tomar-se $\delta_{2}>0$ pequeno o suficiente para que se o campo descontínuo $Z^{*}$ satisfizer $\left|Z-Z^{*}\right|<\delta_{2}$, decorrerá que a aplicação de Poincaré $\eta^{*}$ associada a $Z^{*}$ satisfaz $\left|\eta-\eta^{*}\right|<\mathrm{k}$. Portanto $\eta^{*}$ possui um ponto fixo hiperbólico $\mathrm{p}^{*}$ que corresponde a uma poli-trajetória fechada $\gamma^{*}$ elementar de $Z^{*}$. Devido à continuidade das funções envolvidas, se $\delta_{2}$ for tomado pequeno o suficiente, $\gamma^{*}$ só cruzará a curva $\mathrm{D}$ em pontos de costura, caracterizando que $\gamma^{*}$ é do tipo I.

Suponha-se que $\gamma$ é uma poli-trajetória fechada elementar tipo II de Z. Logo, $\gamma=\mathrm{D}$ e para qualquer $\mathrm{p} \in \mathrm{D}, \mathrm{X} f(\mathrm{p}) \mathrm{Y} f(\mathrm{p})<0$ e $\operatorname{det}[\mathrm{X}, \mathrm{Y}](\mathrm{p}) \neq 0$. Como $\mathrm{D}$ é compacto e as funções envolvidas são contínuas, existe $\delta_{2}>0$ tal que se o campo descontínuo $Z^{*}$ é tal que $\left|Z-Z^{*}\right|<\delta_{2}$, então $\mathrm{X}^{*} f(\mathrm{p}) \mathrm{Y}^{*} f(\mathrm{p})<0$ e $\operatorname{det}\left[\mathrm{X}^{*}, \mathrm{Y}^{*}\right](\mathrm{p}) \neq 0$. Logo, pela definição, $\gamma^{*}=\mathrm{D}$ é uma poli-trajetória fechada elementar tipo II de $Z^{*}$.

Suponha-se que $\gamma$ é uma poli-trajetória fechada elementar tipo III de $Z$. Pela continuidade das funções envolvidas, é claro que existe $\delta_{2}>0$ tal que se $Z^{*}$ dista menos que $\delta_{2}$ de $Z$, então $Z^{*}$ possui um ponto dobra próximo ao ponto dobra da órbita $\gamma$ e seguindo a órbita que passa por este 
ponto dobra, como ela está próxima da órbita $\gamma$, a mesma acaba entrando num arco de $\mathrm{F}_{\mathrm{Z}}$, formando uma poli-trajetória fechada $\gamma^{*}$.

Como o número de poli-trajetórias fechadas de $Z$ é finito (pois são todas elementares), pode-se tomar $\delta_{2}$ estritamente positivo e pequeno o suficiente para que $Z^{*}$ possua apenas as poli-trajetórias fechadas associadas às órbitas de $Z$ e sejam todas elementares.

Portanto $Z^{*}$ pertence a $G^{r}(2)$.

- Afirma-se que existe $\delta_{3}>0$ tal que se $\left|Z-Z^{*}\right|<\delta_{3}$, então $Z^{*} \in G^{\mathrm{r}}(3)$.

De fato, como $Z=(X, Y) \in G^{r}(3)$, então $\left.X\right|_{N}$ e $\left.Y\right|_{s} \in \Sigma^{r}$, e se $p \in$ $D \cap \partial M$, então $X \beta(p) Y \beta(p) \neq 0$, e caso esta última expressão seja negativa, tem-se $\mathrm{X} \beta(\mathrm{p}) \mathrm{Y} f(\mathrm{p})-\mathrm{X} f(\mathrm{p}) \mathrm{Y} \beta(\mathrm{p}) \neq 0$. É imediato pela continuidade das funções envolvidas que existe $\delta_{3}>0$ tal que se $\left|Z-Z^{*}\right|<\delta_{3}$, então $X^{*} \beta(p) Y^{*} \beta(p) \neq 0$, e caso esta última expressão seja negativa, $\mathrm{X}^{*} \beta(\mathrm{p}) \mathrm{Y} f^{*}(\mathrm{p})-\mathrm{X}^{*} f(\mathrm{p}) \mathrm{Y}^{*} \beta(\mathrm{p}) \neq 0$.

Portanto $Z^{*}$ pertence a $\mathrm{G}^{\mathrm{r}}(3)$.

- Afirma-se que existe $\delta_{4}>0$ tal que se $\left|Z-Z^{*}\right|<\delta_{4}$, então $Z^{*} \in G^{r}(4)$.

De fato, como $Z=(X, Y) \in G^{r}(4)$, então $\left.X\right|_{N}$ e $\left.Y\right|_{s} \in \sum^{r}$, e portanto existe $\eta>0$ tal que se $\left|Z-Z^{*}\right|<\eta$, tem-se $\left.X^{*}\right|_{N}$ e $\left.Y^{*}\right|_{S} \in \Sigma^{r}$. Logo $X^{*}$ e $Y^{*}$ não possuem conexões de separatrizes em $N$ e $S$, respectivamente, restando apenas analisar-se o possível aparecimento de conexões de separatrizes que possuem pelo menos um ponto da curva de descontinuidade D.

Algumas das possíveis configurações do campo $Z$ estão exemplificadas nas figuras abaixo:

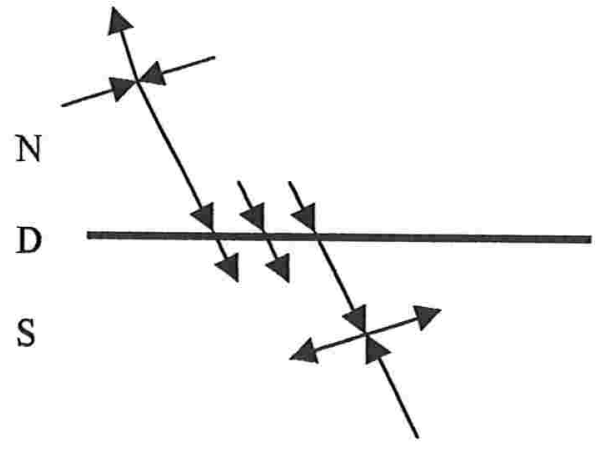

Fig. 63

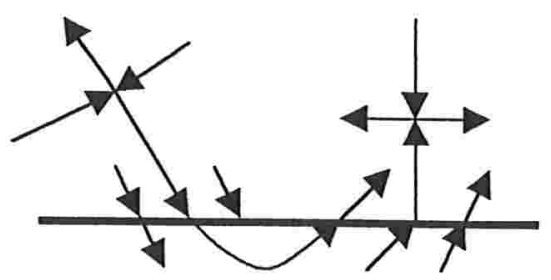

Fig. 64 


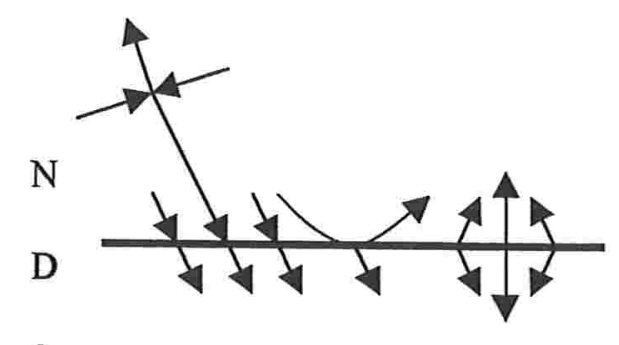

S

Fig. 65

Sabe-se que o número de separatrizes de $Z$ é finito, e $Z$ não possui conexão de separatrizes.

Sejam $\gamma_{1}, \gamma_{2}, \ldots, \gamma_{n}$ as separatrizes de $X, \gamma_{n+1}, \ldots, \gamma_{m}$ as separatrizes de $\mathrm{Y}$ e $\gamma_{\mathrm{m}+1}, \ldots, \gamma_{\mathrm{k}}$ as separatrizes de $\mathrm{F}_{\mathrm{Z}}$. É possivel medir a distância entre cada uma destas separatrizes. Seja $\lambda=\min \left\{\operatorname{dis}\left(\gamma_{\mathrm{i}}, \gamma_{\mathrm{j}}\right) \mid \mathrm{i} \neq \mathrm{j}\right\}$. É claro que $\lambda>0$, pois $Z$ não possui conexão de separatrizes e o número de separatrizes é finito.

Seja $\delta_{4}=\min \{\lambda, \eta\}$, então se $\left|Z-Z^{*}\right|<\delta_{4}$, tem-se que $Z^{*}$ não possui conexão de separatrizes.

Portanto $Z^{*}$ pertence a $G^{\mathrm{r}}(4)$.

Para completar-se a demonstração da Proposição, toma-se $\delta=\min \left\{\delta_{1}, \delta_{2}, \delta_{3}, \delta_{4}\right\}$.

É claro que $\delta>0$ e se $Z^{*}=\left(X^{*}, Y^{*}\right) \in \Omega^{r}$ é tal que $\left|Z-Z^{*}\right|_{\mathrm{r}}=$ $\max \left\{\left|\mathrm{X}-\mathrm{X}^{*}\right|_{\mathrm{r}},\left|\mathrm{Y}-\mathrm{Y}^{*}\right|_{\mathrm{r}}\right\}<\delta$, então $Z^{*} \in \mathrm{G}^{\mathrm{r}}(\mathrm{M})$, pois $\mathrm{Z}^{*}$ pertence aos conjuntos $\mathrm{G}^{\mathrm{r}}(1), \mathrm{G}^{\mathrm{r}}(2), \mathrm{G}^{\mathrm{r}}(3)$ e $\mathrm{G}^{\mathrm{r}}(4)$.

Logo, o conjunto $\mathrm{G}^{\mathrm{r}}(\mathrm{M})$ é aberto em $\Omega^{\mathrm{r}}(\mathrm{M}, f)$.

\subsection{Definição}

Seja $Z \in \Omega^{\mathrm{r}}(\mathrm{M}, f)$. Para cada par $(\theta, \mathrm{v}) \in \mathfrak{R}^{2} \times \mathfrak{R}^{2}$, define-se o campo vetorial descontínuo $Z_{\theta, v}$ como o campo $Z$ transladado de $\mathrm{v}=\left(\mathrm{v}_{1}, \mathrm{v}_{2}\right)$ e rodado por ângulos $\theta=\left(\theta_{1}, \theta_{2}\right)$, isto é,

onde

$$
Z_{\theta, v}=R_{\theta}(Z+v)=\left(R_{\theta 1}(X+v), R_{\theta 2}(Y+v)\right)
$$

e

$$
R_{\theta 1}(X+v)=\left[\begin{array}{cc}
\cos \theta_{1} & -\operatorname{sen} \theta_{1} \\
\operatorname{sen} \theta_{1} & \cos \theta_{1}
\end{array}\right]\left[\begin{array}{l}
X_{1}+v_{1} \\
X_{2}+v_{2}
\end{array}\right]
$$




$$
\mathrm{R}_{\theta 2}(\mathrm{Y}+\mathrm{v})=\left[\begin{array}{rr}
\cos \theta_{2} & -\operatorname{sen} \theta_{2} \\
\operatorname{sen} \theta_{2} & \cos \theta_{2}
\end{array}\right]\left[\begin{array}{l}
Y_{1}+v_{1} \\
Y_{2}+v_{2}
\end{array}\right]
$$

Recorda-se aqui o teorema da densidade por translação e rotação do caso contínuo [S2].

\subsection{Teorema}

Sejam $M$ uma região compacta de $\Re^{2}$ com fronteira de classe $C^{2}$ e $X$ um campo vetorial de classe $C^{l}$ em $\Re^{2}$. O conjunto $\mathscr{X}_{1}(X)=\left\{(\theta, v) \in \Re x \Re^{2} \mid\right.$ $\left.R_{\theta}(X) \notin \Sigma^{r}\right\}$ tem medida nula em $\Re^{3}$.

\subsection{Teorema}

Seja $Z \in \Omega^{r}(M, f)$. O conjunto $\left\{(\theta, v) \in \Re^{2}{ }^{2} \Re^{2} / Z_{\theta, v} \notin G^{r}\right\}$ tem medida nula em $\Re^{4}$.

demonstração

Seja $Z \in \Omega^{\mathrm{r}}(\mathrm{M}, f)$.

Seja crit(Z) o conjunto dos valores críticos de $Z$, isto é,

$\operatorname{crit}(Z)=\left\{v \in \mathfrak{R}^{2} \mid \exists p \in N \operatorname{com} X(p)=v e \operatorname{det}(D X(p))=0\right.$, ou $\exists \mathrm{p} \in \mathrm{S} \operatorname{com} \mathrm{Y}(\mathrm{p})=\mathrm{v}$ e $\operatorname{det}(\mathrm{DY}(\mathrm{p}))=0\}$.

Pelo Teorema de Sard (Teorema 3.7), o conjunto crit $(Z)$ tem medida de Lebesgue nula em $\mathfrak{R}^{2}$.

É claro que os conjuntos $\mathrm{X}(\partial \mathrm{N})$ e $\mathrm{Y}(\partial \mathrm{S})$ também têm medida nula, pois são imagens contidas em $\mathfrak{R}^{2}$ de conjuntos uni-dimensionais por funções contínuas. Observa-se que $\partial \mathrm{N} \cup \partial \mathrm{S}=\partial \mathrm{M} \cup \mathrm{D}$.

Portanto o conjunto $C(Z)=\operatorname{crit}(Z) \cup X(\partial N) \cup Y(\partial S)$ tem medida nula.

Se $v \in \mathfrak{R}^{2}$ é tal que $-\mathrm{v} \notin \mathrm{C}(Z)$, então $X+\mathrm{v}$ e $\mathrm{Y}+\mathrm{v}$ têm todos os seus pontos singulares simples e no interior de $\mathrm{N}$ e $\mathrm{S}$, respectivamente.

Será provado que se $-\mathrm{v} \notin \mathrm{C}(\mathrm{Z})$, então o conjunto $\Psi_{1}(Z, v)=\left\{\theta \in \mathfrak{R}^{2} \mid\right.$ $\left.\mathrm{R}_{\theta}(Z+v) \notin \mathrm{G}^{\mathrm{r}}\right\}$ tem medida nula em $\mathfrak{R}^{2}$. Como $\Psi_{1}(Z, v)=\cup \Psi_{1}{ }^{\mathrm{i}}(Z, v), \mathrm{i}=1$, .., 4, onde $\Psi_{1}{ }^{i}(Z, v)=\left\{\theta \in \Re^{2} \mid R_{\theta}(Z+v) \notin G^{r}(i)\right\}$, a prova será feita mostrando-se que cada um dos conjuntos $\Psi_{1}{ }^{i}(Z, v)$ tem medida nula em $\mathfrak{R}^{2}$. 
- $\Psi_{1}{ }^{1}(Z, v)=\left\{\theta \in \mathfrak{R}^{2} \mid \mathrm{R}_{\theta}(Z+\mathrm{v}) \notin \mathrm{G}^{\mathrm{r}}(1)\right\}$ tem medida nula em $\mathfrak{R}^{2}$.

Sem perda de generalidade, pode-se supor que $v=(0,0)$, isto é, $Z+$ $\mathrm{v}=\mathrm{Z}=(\mathrm{X}, \mathrm{Y})=((\mathrm{a}, \mathrm{b}),(\mathrm{c}, \mathrm{d}))$. Esta suposição será usada também na demonstração dos outros itens.

Logo, $R_{\theta}(Z+v)=R_{\theta}(Z)=\left(X_{\theta 1}, Y_{\theta 2}\right)=\left(\left(a \cos \theta_{1}-b \operatorname{sen} \theta_{1}, a \operatorname{sen} \theta_{1}+\right.\right.$ $\left.\left.\mathrm{b} \cos \theta_{1}\right),\left(\mathrm{c} \cos \theta_{2}-\mathrm{d} \operatorname{sen} \theta_{2}, c \operatorname{sen} \theta_{2}+\mathrm{d} \cos \theta_{2}\right)\right)(1)$.

Pelo Teorema 3.11, $\left\{\theta \in \Re^{2} \mid \mathrm{R}_{\theta}(\mathrm{X}) \notin \Sigma^{\mathrm{r}}(1)\right\} \cup\left\{\theta \in \mathfrak{R}^{2} \mid \mathrm{R}_{\theta}(\mathrm{Y})\right.$ $\left.\notin \Sigma^{\mathrm{r}}(1)\right\}$ tem medida nula. Logo, resta apenas fazer a prova para as $D$ singularidades.

O campo de Filippov $F_{Z \theta}$ associado ao campo vetorial $Z_{\theta}$ possui uma singularidade no ponto $\mathrm{p} \in \mathrm{D}$ se

$$
\mathrm{X}_{\theta 1} f(\mathrm{p}) \cdot \mathrm{Y}_{\theta 2} f(\mathrm{p})=\left(\mathrm{a} \operatorname{sen} \theta_{1}+\mathrm{b} \cos \theta_{1}\right) \cdot\left(\mathrm{c} \operatorname{sen} \theta_{2}+\mathrm{d} \cos \theta_{2}\right)<0
$$

e $\operatorname{det}\left[X_{\theta 1}, Y_{\theta 2}\right](p)=(a c+b d) \operatorname{sen}\left(\theta_{2}-\theta_{1}\right)+(a d-b c) \cos \left(\theta_{2}-\theta_{1}\right)=0$ (3).

E esta singularidade será não-hiperbólica se

$\mathrm{d}\left(\left.\operatorname{det}\left[\mathrm{X}_{\theta 1}, \mathrm{Y}_{\theta 2}\right]\right|_{\mathrm{D}}\right)(\mathrm{p})=(\partial / \partial \mathrm{x})\left(\operatorname{det}\left[\mathrm{X}_{\theta 1}, \mathrm{Y}_{\theta 2}\right]\right)(\mathrm{p})=\left(\mathrm{a}_{\mathrm{x}} \mathrm{c}+\mathrm{ac}_{\mathrm{x}}+\mathrm{b}_{\mathrm{x}} \mathrm{d}+\right.$ $\left.b d_{x}\right) \operatorname{sen}\left(\theta_{2}-\theta_{1}\right)+\left(a_{x} d+a d_{x}-b_{x} c-b c_{x}\right) \cos \left(\theta_{2}-\theta_{1}\right)=0$ (4).

Analisando-se a expressão (3), tem-se

$(3) \Rightarrow(a c+b d) \operatorname{sen}\left(\theta_{2}-\theta_{1}\right)=-(a d-b c) \cos \left(\theta_{2}-\theta_{1}\right)$

Pela primeira parte da demonstração deste teorema, os campos vetoriais $X$ e $Y$ não possuem singularidades em $D, \log 0 X(p)=(a(p), b(p))$ $\neq(0,0)$ e $Y(p)=(c(p), d(p)) \neq(0,0)$, portanto $a(p) d(p)-b(p) c(p) \neq 0$ e/ou $a(p) c(p)+b(p) d(p) \neq 0$. Pode-se supor, sem perda de generalidade, que $a(p) c(p)+b(p) d(p) \neq 0$.

Logo, no ponto $\mathrm{p}$, tem-se

$$
\begin{aligned}
& (5) \Rightarrow \frac{\operatorname{sen}\left(\theta_{2}-\theta_{1}\right)}{\cos \left(\theta_{2}-\theta_{1}\right)}=-\frac{(a d-b c)}{(a c+b d)} \\
& \Rightarrow \quad \operatorname{tg}\left(\theta_{2}-\theta_{1}\right)=-\frac{(a d-b c)}{(a c+b d)}
\end{aligned}
$$




$$
\Rightarrow \quad \theta_{2}-\theta_{1}-\mathrm{k} \pi=\operatorname{arctg}\left[-\frac{(\mathrm{ad}-\mathrm{bc})}{(\mathrm{ac}+\mathrm{bd})}\right]
$$

Seja $\theta_{2}\left(p, k, \theta_{1}\right)=\theta_{0}(p)+\theta_{1}+k \pi$,

$$
\text { onde } \theta_{0}(p)=\operatorname{arctg}\left[-\frac{(a(p) d(p)-b(p) c(p))}{(a(p) c(p)+b(p) d(p))}\right] \text {. }
$$

As singularidades de $F_{Z \theta}$ ocorrerão para $\theta_{2}=\theta_{2}\left(p, k, \theta_{1}\right)$ e $k$ par ou $\mathrm{k}$ ímpar. Isto é porque uma das paridades do $\mathrm{k}$ corresponde a pontos $\mathrm{da}$ região de costura. Pode-se supor, sem perda de generalidade, que as singularidades ocorram para $\mathrm{k}$ par. Os valores críticos da aplicação $\theta_{2}$ corresponderão aos pontos críticos não hiperbólicos. Estes valores serão calculados a seguir.

$$
\begin{aligned}
& \frac{\partial \theta_{2}}{\partial p}\left(p, k, \theta_{1}\right)=\frac{\partial \theta_{0}}{\partial p}(p)=\frac{\partial \theta_{0}}{\partial x}(x, 0)=
\end{aligned}
$$

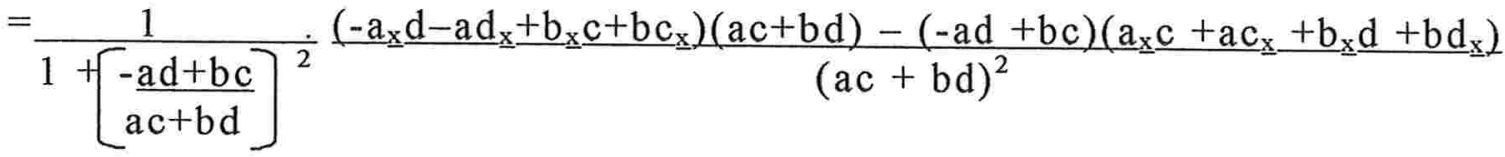

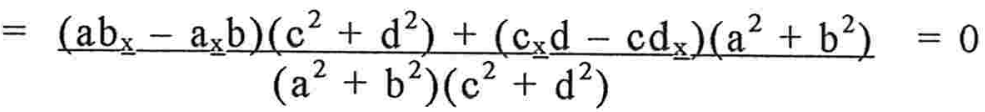

Pelo Teorema de Sard (Teorema 3.7), estes valores críticos formam um conjunto de medida nula.

Tendo sido provado que o conjunto de campos descontínuos que possui uma singularidade não-hiperbólica do campo de Filippov tem medida nula, resta mostrar que o conjunto dos campos descontínuos tais que $\mathrm{X}$ ou $\mathrm{Y}$ possui uma tangência com $\mathrm{D}$ de ordem maior que dois também possui medida nula.

Lembra-se que o ponto $\mathrm{p} \in \mathrm{D}$ é uma $\mathrm{D}$-dobra se $\mathrm{Z}_{\theta, \mathrm{v}}$ se $\mathrm{Y}_{\theta 2} f(\mathrm{p}) \neq 0$, $\mathrm{X}_{\theta 1} f(\mathrm{p})=0$ e $\mathrm{X}^{2}{ }_{\theta 1} f(\mathrm{p}) \neq 0$, ou $\mathrm{X}_{\theta 1} f(\mathrm{p}) \neq 0, \mathrm{Y}_{\theta 2} f(\mathrm{p})=0$ e $\mathrm{Y}^{2}{ }_{\theta 2} f(\mathrm{p}) \neq 0$.

Para que uma tangência dos campos vetoriais $X_{\theta 1}$ ou $Y_{\theta 2} \operatorname{com} D$ não seja regular, deve-se ter $\mathrm{X}_{\theta 1} f(\mathrm{p})=\mathrm{Y}_{\theta 2} f(\mathrm{p})=0$, ou $\mathrm{X}_{\theta 1} f(\mathrm{p})=\mathrm{X}_{\theta 1}^{2} f(\mathrm{p})=0$, ou $\mathrm{Y}_{\theta 2} f(\mathrm{p})=\mathrm{Y}^{2}{ }_{\theta 2} f(\mathrm{p})=0$.

Na notação usada, tem-se

$$
\mathrm{X}_{\theta 1} f(\mathrm{p})=\mathrm{a} \operatorname{sen} \theta_{1}+\mathrm{b} \cos \theta_{1}
$$


$\mathrm{X}^{2}{ }_{\theta 1} f(\mathrm{p})=\left(\mathrm{a}_{\mathrm{x}} \operatorname{sen} \theta_{1}+\mathrm{b}_{\mathrm{x}} \cos \theta_{1}, \mathrm{a}_{\mathrm{y}} \operatorname{sen} \theta_{1}+\mathrm{b}_{\mathrm{y}} \cos \theta_{1}\right) \cdot(0,1)=$ $=a_{y} \operatorname{sen} \theta_{1}+b_{y} \cos \theta_{1}$

$\mathrm{Y}_{\theta 2} f(\mathrm{p})=\mathrm{c} \operatorname{sen} \theta_{2}+\mathrm{d} \cos \theta_{2}$

$\mathrm{Y}^{2}{ }_{\theta 2} f(\mathrm{p})=\left(\mathrm{c}_{\mathrm{x}} \operatorname{sen} \theta_{2}+\mathrm{d}_{\mathrm{x}} \cos \theta_{2}, \mathrm{c}_{\mathrm{y}} \operatorname{sen} \theta_{2}+\mathrm{d}_{\mathrm{y}} \cos \theta_{2}\right) \cdot(0,1)=$ $=c_{y} \operatorname{sen} \theta_{2}+d_{y} \cos \theta_{2}$

Da equação $\mathrm{X}_{\theta 1} f(\mathrm{p})=0$, tem-se

$$
\mathrm{a} \operatorname{sen} \theta_{1}+\mathrm{b} \cos \theta_{1}=0
$$

$\Rightarrow \quad \frac{\operatorname{sen} \theta_{1}}{\cos \theta_{1}}=\frac{-b}{a}$, se $a \neq 0$

$\Rightarrow \quad \operatorname{tg} \theta_{1}=\frac{-b}{a}$

$\Rightarrow \quad \theta_{1}= \begin{cases}\operatorname{arctg}\left[\frac{-b}{a}\right]+k \pi, & \text { se } a \neq 0 \\ \frac{\pi}{2}+k \pi, & \text { se } a=0\end{cases}$

Da equação $\mathrm{X}^{2}{ }_{\theta 1} f(\mathrm{p})=0$, tem-se

$$
\theta_{1}= \begin{cases}\operatorname{arctg}\left[\frac{-b_{y}}{a_{y}}\right]+k^{\prime} \pi, & \text { se } a_{y} \neq 0 \\ \frac{\pi}{2}+k^{\prime} \pi, & \text { se } a_{y}=0\end{cases}
$$

Da equação $\mathrm{Y}_{\theta 2} f(\mathrm{p})=0$, tem-se

$$
\theta_{2}= \begin{cases}\operatorname{arctg}\left[\frac{-d}{c}\right]+k \pi, & \text { se } c \neq 0 \\ \frac{\pi}{2}+k \pi, & \text { se } c=0\end{cases}
$$

Da equação $\mathrm{Y}^{2}{ }_{\theta 2} f(\mathrm{p})=0$, tem-se

$$
\theta_{2}=\left\{\begin{array}{lc}
\operatorname{arctg}\left[\frac{-d_{y}}{c_{y}}\right]+k^{\prime} \pi, \text { se } c_{y} \neq 0 \\
\frac{\pi}{2}+k^{\prime} \pi, & \text { se } c_{y}=0
\end{array}\right.
$$


O que se deseja evitar é que ocorram simultaneamente as situações (4) e (5), ou (4) e (6), ou (6) e (7).

Acontecerão ao mesmo tempo as situações (4) e (5) num ponto $\mathrm{p} \in \mathrm{D}$ se

$$
\begin{aligned}
& \theta_{1}=\operatorname{arctg}\left[\frac{-b}{a}\right]+k \pi=\operatorname{arctg}\left[\frac{-\underline{b}_{y}}{a_{y}}\right]+k^{\prime} \pi \\
& \Rightarrow \frac{-b}{a}=\frac{-b_{y}}{a_{y}}+k^{\prime \prime} \pi .
\end{aligned}
$$

Mas, para cada valor de $\mathrm{p}$ que esta situação ocorre, os valores de $\theta_{1}$ que devem ser evitados estão espaçados de $\pi$, ou seja, formam um conjunto enumerável.

Se $a(p)=a_{y}(p)=0$, o que deve ser evitado são os $\theta_{1}{ }^{\prime}$ s da forma $\theta_{1}=\pi / 2+\mathrm{k} \pi$, que também formam um conjunto enumerável.

Para as situações (6) e (7), o raciocínio é análogo.

Resta verificar quando as situações (4) e (6) ocorrem simultaneamente.

Se $a(p) \neq 0$ e $c(p) \neq 0$, os valores $\Theta=\left(\theta_{1}, \theta_{2}\right)$ que devem ser evitados podem ser dados pela função

$$
\Theta\left(\mathrm{p}, \mathrm{k}, \mathrm{k}^{\prime}\right)=\left(\operatorname{arctg}(-\mathrm{b}(\mathrm{p}) / \mathrm{a}(\mathrm{p}))+\mathrm{k} \pi, \operatorname{arctg}(-\mathrm{d}(\mathrm{p}) / \mathrm{c}(\mathrm{p}))+\mathrm{k}^{\prime} \pi\right)
$$

Para k e k' fixos, a função $\Theta$ é uma função de $\Re$ em $\Re^{2}$, portanto sua imagem tem no máximo dimensão $1, \log$ o tem medida de Lebesgue nula em $\Re^{2}$. O par $\left(k, k^{\prime}\right) \in \mathbf{Z}^{2}$, portanto os gráficos de $\Theta\left(p, k, k^{\prime}\right)$ são translações do gráfico de $\Theta(p, 0,0)$ espaçadas de $\pi$. Como reunião enumerável de conjuntos de medida nula também possui medida nula, pode-se concluir que os valores de $\Theta=\left(\theta_{1}, \theta_{2}\right)$ tal que $Z_{\theta}$ possui alguma tangência com $D$ que não seja uma $\mathrm{D}$-dobra formam um conjunto de medida nula. $\mathfrak{R}^{2}$.

Logo, $\Psi_{1}{ }^{1}(Z, v)=\left\{\theta \in \Re^{2} \mid R_{\theta}(Z+v) \notin G^{r}(1)\right\}$ tem medida nula em

- $\Psi_{1}^{2}(Z, v)=\left\{\theta \in \mathfrak{R}^{2} \mid R_{\theta}(Z+v) \notin G^{r}(2)\right\}$ tem medida nula em $\mathfrak{R}^{2}$.

Pelo Teorema 3.11, $\left\{\theta \in \mathfrak{R}^{2} \mid \mathrm{R}_{\theta}(\mathrm{X}) \notin \Sigma^{\mathrm{r}}(2)\right\} \cup\left\{\theta \in \mathfrak{R}^{2} \mid \mathrm{R}_{\theta}(\mathrm{Y}) \notin\right.$ $\left.\Sigma^{\mathrm{r}}(2)\right\}$ tem medida nula. Logo, resta apenas fazer a prova para as politrajetórias fechadas. 
Dado $\theta^{*}=\left(\theta_{1}, \theta_{2}\right)$, suponhamos que $\gamma^{*}$ seja uma poli-trajetória fechada não elementar do campo $\mathrm{R}_{\theta^{*}}(Z+v)$. Então $\gamma^{*}$ é do tipo 1 e $\eta^{\prime}(p)=1$ ou $\gamma^{*}$ é do tipo III e possui apenas arcos de trajetórias dos campos $\mathrm{X}+\mathrm{v}$ e $\mathrm{Y}+\mathrm{v}$ e um ponto D-dobra ou $\gamma^{*}$ é do tipo III e possui pontos do arco de deslizamento e do arco de escape.

Afirma-se que qualquer um dos casos acima só ocorre para valores de $\theta^{*}$ isolados em $\mathfrak{R}^{2}$. contínuo.

De fato, se $\gamma^{*}$ é do tipo I e $\eta^{\prime}(\mathrm{p})=1$, a análise é análoga à do caso

Se $\gamma^{*}$ é do tipo III e possui apenas arcos de trajetórias dos campos $\mathrm{X}+\mathrm{v}$ e $\mathrm{Y}+\mathrm{v}$ e um ponto $\mathrm{D}$-dobra, para valores de $\theta$ próximos a $\theta^{*}$, o campo $\mathrm{R}_{\theta}(Z+v)$ possui uma poli-trajetória fechada $\gamma$ próxima a $\gamma^{*}$ tal que $\gamma$ é elementar do tipo III ou do tipo I, ou é uma órbita periódica de $\mathrm{X}$ ou de $\mathrm{Y}$. Isto é, $\gamma$ não tangencia mais a curva $\mathrm{D}$ ou $\gamma$ possui um arco de trajetória de $\mathrm{F}_{Z}$.

Se $\gamma^{*}$ é do tipo III e possui pontos do arco de deslizamento e do arco de escape então $\gamma^{*}$ possui uma conexão de D-dobras, através de arcos de trajetória de $\mathrm{X}+\mathrm{v}$ e de $\mathrm{Y}+\mathrm{v}$. Esta situação pode ser evitada para valores de $\theta$ próximos a $\theta^{*}$, da mesma forma como podem ser evitadas as conexões de tangências, a serem vistas no final da demonstração deste teorema.

Se $\gamma^{*}$ é uma poli-trajetória fechada que tangencia $\partial \mathrm{M}$, isto deve estar acontecendo de maneira que as tangências estejam ocorrendo transversalmente ao $\partial \mathrm{M}$ no espaço $\mathfrak{R}^{2}{ }_{\theta} \mathrm{xM}$. Caso contrário, as tangências podem ser evitadas com a soma do vetor $\mathrm{v}$. $\mathfrak{R}^{2}$

Logo, $\Psi_{1}{ }^{2}(Z, v)=\left\{\theta \in \mathfrak{R}^{2} \mid \mathrm{R}_{\theta}(Z+v) \notin \mathrm{G}^{\mathrm{r}}(2)\right\}$ tem medida nula em

- $\Psi_{1}{ }^{3}(Z, v)=\left\{\theta \in \mathfrak{R}^{2} \mid \mathrm{R}_{\theta}(Z+\mathrm{v}) \notin \mathrm{G}^{\mathrm{r}}(3)\right\}$ tem medida nula em $\mathfrak{R}^{2}$.

Pelo Teorema 3.11, $\left\{\theta \in \mathfrak{R}^{2} \mid \mathrm{R}_{\theta}(\mathrm{X}) \notin \Sigma^{\mathrm{r}}(3)\right\} \cup\left\{\theta \in \mathfrak{R}^{2} \mid \mathrm{R}_{\theta}(\mathrm{Y})\right.$ $\left.\notin \Sigma^{\mathrm{r}}(3)\right\}$ tem medida nula. Logo, resta apenas fazer a prova para as tangências que surgirem em $\mathrm{D} \cap \partial \mathrm{M}$.

Deve-se mostrar que para todo ponto $p \in D \cap \partial M$ e quase todo $\theta$ fixo, tem-se $X_{\theta 1} \beta(p)$. $Y_{\theta 2} \beta(p) \neq 0$, para garantir que não há singularidade de $R_{\theta}(Z+v)$ em $\partial M$ e que $p$ não é um ponto de tangência com a fronteira de $\mathrm{X}$ ou de $\mathrm{Y}$. 
Deve-se provar também que se $X_{\theta 1} \beta(p)$. $Y_{\theta 2} \beta(p)<0$, tem-se que $X_{\theta 1} \beta(p) . Y_{\theta 2} f(p)-X_{\theta 1} f(p) . Y_{\theta 2} \beta(p) \neq 0$. Isto garantirá que se surgir uma tangência em $p$ na regularização do campo $R_{\theta}(Z+v)$, esta tangência será quadrática.

Pode-se supor, sem perda de generalidade, que $\beta(x, y)=x$.

Para que $X_{\theta 1} \beta(p)=0$, deve-se ter

$X_{\theta 1} \beta(p)=a \cos \theta_{1}-b \operatorname{sen} \theta_{1}=0$

$\Rightarrow \quad \frac{\mathrm{a}}{\mathrm{b}}=\frac{\underline{\operatorname{sen} \theta_{1}}}{\cos \theta_{1}}=\operatorname{tg} \theta_{1}$

$\Rightarrow \quad \theta_{1}=\operatorname{arctg}(\mathrm{a} / \mathrm{b})$

ou $\quad \theta_{1}=\operatorname{arccotg}(\mathrm{b} / \mathrm{a})$.

Analogamente, para se ter $Y_{\theta 2} \beta(p)=0$, deve-se ter

$$
\theta_{2}=\operatorname{arctg}(\mathrm{c} / \mathrm{d})
$$

É claro que (8) ou (9) só acontecerão para um conjunto de medida nula de $\theta=\left(\theta_{1}, \theta_{2}\right)$.

Caso $X_{\theta 1} \beta(p) . Y_{\theta 2} \beta(p)<0$, tem-se

$X_{\theta 1} \beta(p) . Y_{\theta 2} f(p)-X_{\theta 1} f(p) . Y_{\theta 2} \beta(p)=$

$=\left(\mathrm{a} \cos \theta_{1}-\mathrm{b} \operatorname{sen} \theta_{1}\right)\left(\mathrm{c} \operatorname{sen} \theta_{2}+\mathrm{d} \cos \theta_{2}\right)-\left(\mathrm{a} \operatorname{sen} \theta_{1}+\mathrm{b} \cos \theta_{1}\right)$ $\left(c \cos \theta_{2}-d \operatorname{sen} \theta_{2}\right)=$

$=a c \cos \theta_{1} \operatorname{sen} \theta_{2}+a d \cos \theta_{1} \cos \theta_{2}-b c \operatorname{sen} \theta_{1} \operatorname{sen} \theta_{2}-b d \operatorname{sen} \theta_{1} \cos \theta_{2}-$ ac $\operatorname{sen} \theta_{1} \cos \theta_{2}+a d \operatorname{sen} \theta_{1} \operatorname{sen} \theta_{2}-b c \cos \theta_{1} \cos \theta_{2}+b d \cos \theta_{1} \operatorname{sen} \theta_{2}=$

$=a c \operatorname{sen}\left(\theta_{2}-\theta_{1}\right)+a d \cos \left(\theta_{2}-\theta_{1}\right)-b c \cos \left(\theta_{2}-\theta_{1}\right)+b d \operatorname{sen}\left(\theta_{2}-\theta_{1}\right)=$

$=(a c+b d) \operatorname{sen}\left(\theta_{2}-\theta_{1}\right)+(a d-b c) \cos \left(\theta_{2}-\theta_{1}\right)$.

Como $X(p) \neq 0$ e $Y(p) \neq 0$, as expressões $(a c+b d)$ e $(a d-b c)$ não se anulam simultaneamente em $p, \log$, para cada $\theta_{1}$ fixo, a expressão (10) só se anula para um conjunto discreto de valores de $\theta_{2}$. O mesmo vale se tivermos $\theta_{2}$ fixo e variarmos $\theta_{1}$. Portanto, é imediato que a expressão (10) só se anula para um conjunto de medida nula de $\theta=\left(\theta_{1}, \theta_{2}\right)$ em $\mathfrak{R}^{2}$. 

$\mathfrak{R}^{2}$

Logo, $\Psi_{1}{ }^{3}(Z, v)=\left\{\theta \in \mathfrak{R}^{2} \mid R_{\theta}(Z+v) \notin G^{r}(3)\right\}$ tem medida nula em

- $\Psi_{1}{ }^{4}(Z, v)=\left\{\theta \in \Re \mid R_{\theta}(Z+v) \notin G^{r}(4)\right\}$ tem medida nula em $\Re^{2}$.

Pelo Teorema 3.11, $\left\{\theta \in \mathfrak{R}^{2} \mid \mathrm{R}_{\theta}(\mathrm{X}) \notin \Sigma^{\mathrm{r}}(4)\right\} \cup\left\{\theta \in \mathfrak{R}^{2} \mid \mathrm{R}_{\theta}(\mathrm{Y})\right.$ $\left.\notin \Sigma^{\mathrm{r}}(4)\right\}$ tem medida nula. Logo, resta apenas considerar-se as conexões de separatrizes que possuem algum ponto de D.

Seja $\gamma$ uma conexão de separatrizes de $Z$ que possui algum ponto de D. Pode-se considerar dois casos:

a) $\gamma$ possui pelo menos um ponto de costura; ou

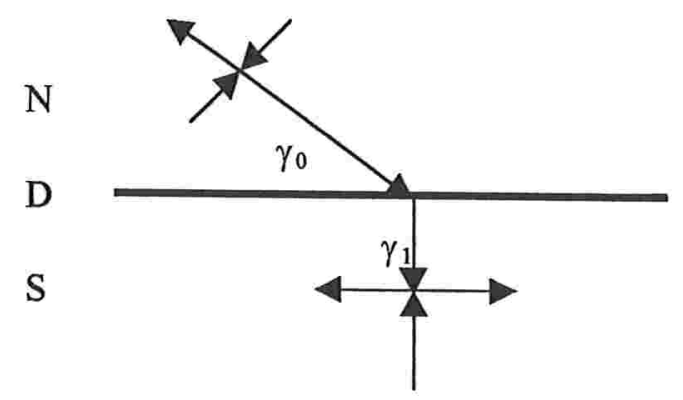

Fig. 66

b) $\gamma$ não possui nenhum ponto de costura. Neste caso, $\gamma$ pode estar conectando uma sela de $\mathrm{F}_{Z}$ com uma singularidade ou tangência de $\mathrm{X}$ ou $Y$ através de um ponto dobra, conforme figura 67, ou $\gamma$ é uma separatriz de $\mathrm{X}$ ou $\mathrm{Y}$ que tende para uma sela de $\mathrm{F}_{Z}$ dentro de $\mathrm{N}$ ou de $S$, respectivamente, conforme mostra a figura 68 .

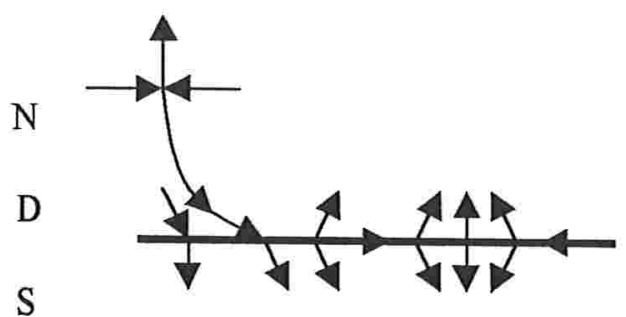

Fig. 67

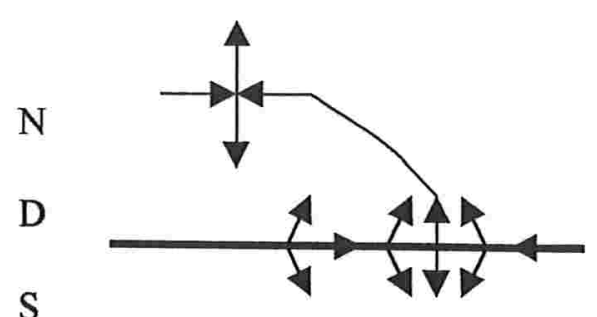

Fig. 68

Seja $\gamma=\gamma_{0} \cup \gamma_{1}$ uma conexão de separatrizes de $Z$, onde $\gamma_{0}$ é uma separatriz da sela $s_{0}$ de $X, \gamma_{1}$ é uma separatriz da sela $s_{1}$ de $Y$ e tal que $\gamma \cap \mathrm{D}=\left\{\mathrm{p}_{0}\right\}$, onde $\mathrm{p}_{0}$ é um ponto de costura, conforme ilustrado na figura 66. 
Mantendo-se $\theta_{2}=0$ fixo, o único valor de $\theta_{1}$ para o qual a conexão continua existindo é $\theta_{1}=0$. Na realidade, para qualquer $\theta_{2}$ fixo, existe no máximo um valor de $\theta_{1}$ para o qual as selas $s_{0}$ e $s_{1}$ continuam conectadas através de um ponto de costura.

Se $\gamma$ não contém nenhum ponto de costura, conforme ilustrado nas figuras 67 e 68, a conexão de separatrizes é ainda mais frágil, e pode ser quebrada usando-se uma rotação $\theta_{1} \neq 0$ do campo $X$.

Do fato de existirem apenas um número finito de selas e tangências, segue que só existem um número finito de separatrizes, e que apenas para um conjunto de medida nula de $\theta=\left(\theta_{1}, \theta_{2}\right)$ haverá uma conexão de separatrizes. $\mathfrak{R}^{2}$

Logo, $\Psi_{1}{ }^{4}(Z, v)=\left\{\theta \in \mathfrak{R}^{2} \mid \mathrm{R}_{\theta}(Z+\mathrm{v}) \notin \mathrm{G}^{\mathrm{r}}(4)\right\}$ tem medida nula em

Logo o conjunto $\Psi_{1}(\mathrm{Z}, \mathrm{v})=\cup \Psi_{1}{ }^{\mathrm{i}}(\mathrm{Z}, \mathrm{v}), \mathrm{i}=1, \ldots, 4$ tem medida nula, pois é união de quatro conjuntos de medida nula.

Do Teorema de Fubini segue que o conjunto $\left\{(\theta, v) \in \mathfrak{R}^{2} \times \mathfrak{R}^{2}\right.$ $\left.Z_{\theta, v} \notin G^{r}\right\}=\Psi_{1}(Z, v) \times \Re^{2} \cup \mathfrak{R}^{2} \times C(Z)$ tem medida nula, e com isto a demonstração está concluída.

\subsection{Corolário \\ $O$ conjunto $G^{r}(M)$ é aberto e denso em $\Omega^{r}(M, f)$. \\ demonstração}

Imediata.

Neste Capítulo foram estabelecidas condições para que a regularização de um campo vetorial descontínuo forneça campos vetoriais de Andronov-Pontrjaguin-Peixoto (A-P-P). Foi provado que o conjunto dos Campos Vetoriais Descontínuos que satisfazem estas condições é um conjunto genérico, dentro do conjunto de Campos Vetoriais Descontínuos. 


\section{Capítulo 3 \\ Introdução ao Estudo das Bifurcações de Campos Vetoriais Descontínuos}

Neste capítulo é iniciado o estudo das Bifurcações dos Campos Vetoriais Descontínuos. Recorda-se que, no caso contínuo, uma bifurcação é um fenômeno que ocorre numa família de campos vetoriais quando se verifica uma mudança brusca no retrato de fase dos elementos da família na passagem do parâmetro que rege a família por um certo valor, chamado ponto de bifurcação. Em outras palavras, se $\lambda$ é o parâmetro que rege a família de campos vetoriais $X_{\lambda}$, diz-se que $\lambda_{0}$ é um ponto de bifurcação se $X_{\lambda 1}$ e $X_{\lambda 2}$ não são topologicamente equivalentes, onde $\lambda_{1}$ e $\lambda_{2}$ estão próximos a $\lambda_{0}$ e $\lambda_{1}<\lambda_{0}<\lambda_{2}$.

No caso dos campos vetoriais descontínuos, será utilizada uma família de campos descontínuos $Z_{\lambda}=\left(X_{\lambda}, Y_{\lambda}\right)$ tal que para um certo valor $\lambda_{0} 0$ campo $Z_{\lambda 0}$ não pertence ao conjunto $G^{r}(M)$. O objetivo é verificar quando isto provoca uma bifurcação na família de campos vetoriais regularizados $Z_{\varepsilon, \lambda}$. Serão analisadas algumas das possibilidades de $Z_{\lambda 0}$ não pertencer a $\mathrm{G}^{\mathrm{r}}(\mathrm{M})$.

\section{Ponto Singular sobre a Curva $D$}

Neste parágrafo será estudada a influência que um ponto singular hiperbólico do campo $\mathrm{X}$ ou do campo $\mathrm{Y}$ sobre a curva $\mathrm{D}$ tem sobre a família de campos vetoriais regularizados. Isto é feito utilizando-se uma família de campos vetoriais descontínuos $Z_{\lambda}=\left(X_{\lambda}, Y_{\lambda}\right)$ onde, para cada $\lambda$, numa vizinhança da curva $D, Y_{\lambda}$ cruza a curva de descontinuidade $D$ transversalmente, e $X_{\lambda}$ possui uma única singularidade hiperbólica. Considera-se que a curva de singularidades de $X_{\lambda}$ no espaço $\mathfrak{R}_{\lambda} \mathrm{xM}$ cruza transversalmente a curva $D$ para $\lambda=0$, no interior de $M$. São analisadas as consequências destas singularidades sobre a família de campos vetoriais regularizados $Z_{\varepsilon, \lambda}$, dependendo do tipo de singularidade de $X_{\lambda}$ e do sentido em que o campo $Y_{\lambda}$ cruza a curva de descontinuidade $D$. Observa-se que em dois dos casos analisados não ocorre um fenômeno de bifurcação. 


\subsection{Formas Normais}

Será considerado que $f(\mathrm{x}, \mathrm{y})=\mathrm{y}$, ou seja, que $\mathrm{D}=\{\mathrm{y}=0 \mid(\mathrm{x}, \mathrm{y}) \in \mathrm{M}\}$ e que o ponto $\mathrm{p}=(0,0) \in \operatorname{Int}(\mathrm{M})$.

Seja $X_{\lambda}$ uma família de campos vetoriais contínuos tal que para cada valor de $\lambda, X_{\lambda}$ possui uma singularidade hiperbólica no ponto $\left(\mathrm{x}_{0}(\lambda), \mathrm{y}_{0}(\lambda)\right)$. A família pode ser escrita da seguinte forma:

$$
\begin{aligned}
x_{\lambda}(x, y)=\left(a(\lambda)\left(x-x_{0}(\lambda)\right)+b(\lambda)\right. & \left(y-y_{0}(\lambda)\right)+R(x, y, \lambda), \\
& \left.c(\lambda)\left(x-x_{0}(\lambda)\right)+d(\lambda)\left(y-y_{0}(\lambda)\right)+s(x, y, \lambda)\right),
\end{aligned}
$$

onde $\mathrm{R}(\mathrm{x}, \mathrm{y}, \lambda)$ e $\mathrm{S}(\mathrm{x}, \mathrm{y}, \lambda)$ são restos de ordem maior ou igual a dois.

Como o ponto $\left(\mathrm{x}_{0}(\lambda), \mathrm{y}_{0}(\lambda)\right)$ é uma singularidade hiperbólica, segue que $\Delta(\lambda)=\operatorname{det}\left(D X_{\lambda}\right)\left(x_{0}(\lambda), y_{0}(\lambda)\right)=a(\lambda) d(\lambda)-b(\lambda) c(\lambda) \neq 0$.

Como se deseja que o cruzamento da curva de singularidades com a curva D seja transversal, deve-se ter que nenhum vetor paralelo ao eixo $x$ seja auto-vetor da singularidade. Ou seja, $\left(\mathrm{DX}_{\lambda}\left(\mathrm{x}_{0}(\lambda), \mathrm{y}_{0}(\lambda)\right)\right)(1,0) \neq \mu(1,0)$, logo $(a(\lambda), c(\lambda)) \neq \mu(1,0)$, e portanto, $c(\lambda) \neq 0$.

A mudança de coordenadas

$$
\begin{aligned}
& \mathrm{x}^{*}=\alpha \mathrm{x}+\beta \mathrm{y} \\
& \mathrm{y}^{*}=\gamma \mathrm{y}
\end{aligned}
$$

onde $\alpha=\mathrm{c}(\lambda), \beta=-\mathrm{a}(\lambda)$ e $\gamma=1$, conserva o eixo $\mathrm{x}$ e leva o campo $\mathrm{X}_{\lambda}$ em

$$
\begin{array}{r}
\mathrm{X}^{*} \lambda\left(\mathrm{x}^{*}, \mathrm{y}^{*}\right)=\left(\mathrm{a}^{2}+\mathrm{bc}-\mathrm{a}^{2} / \mathrm{c}-\mathrm{ad}\right) \mathrm{y}^{*}+(-\mathrm{bc}+\mathrm{ad}) \mathrm{y}_{0}+\mathrm{R}^{*}\left(\mathrm{x}^{*}, \mathrm{y}^{*}, \lambda\right), \\
\left.\mathrm{x}^{*}+(\mathrm{a} / \mathrm{c}+\mathrm{d}) \mathrm{y}^{*}-\mathrm{xc}_{0}-\mathrm{dy_{0 }}+\mathrm{S} *\left(\mathrm{x}^{*}, \mathrm{y}^{*}, \lambda\right)\right),
\end{array}
$$

Definindo $\lambda^{*}=(-b c+a d) y_{0}$, reescalonando o sistema de modo a se ter $\mathrm{c}=1$ e voltando às antigas coordenadas, tem-se

$$
X_{\lambda}(x, y)=(-\Delta y+\lambda+R(x, y, \lambda), x+\sigma y+S(x, y, \lambda))
$$

O último passo seria provar que através de mudanças de variáveis os restos $\mathrm{R}$ e $\mathrm{S}$ podem ser embutidos no sistema e basta se estudar a parte linear, ou seja, a família

$$
\mathrm{X}_{\lambda}(\mathrm{x}, \mathrm{y})=(-\Delta \mathrm{y}+\lambda, \mathrm{x}+\sigma \mathrm{y})
$$


porém estas contas são demasiado longas e serão feitas num próximo trabalho. Nesta tese, a título de exemplos, será utilizada a família acima, com $\Delta \neq 0$.

Como, por hipótese, o campo $Y_{\lambda}$, para cada $\lambda$, deve ser transversal à curva $D$ numa vizinhança de $p=(0,0)$, será considerado que as coordenadas são tais que a família $Y_{\lambda}$ é constante e dada por $Y_{\lambda}(x, y)=(k, 1)$, com $k \neq 0$.

Neste parágrafo será utilizada a família $Z_{\lambda}=\left(X_{\lambda}, Y_{\lambda}\right)$.

\subsection{Lema}

A família $Z_{\lambda}=\left(X_{\lambda}, Y_{\lambda}\right)$ definida acima apresenta os seguintes casos:

(i) $k>0, \Delta<0$ :

sela hiperbólica de $X_{\lambda}+$ nó hiperbólico de $F_{Z}$ quando $\lambda<0$, nenhuma singularidade quando $\lambda>0$.

(ii) $k>0,4 \Delta>\sigma^{2}>0$ :

nó hiperbólico de $F_{Z}$ quando $\lambda<0$,

foco hiperbólico de $X_{\lambda}$ quando $\lambda>0$.

(iii) $k>0, \sigma^{2} \geq 4 \Delta>0$ :

nó hiperbólico de $F_{Z}$ quando $\lambda<0$,

nó hiperbólico de $X_{\lambda}$ quando $\lambda>0$.

(iv) $k<0,4 \Delta>\sigma^{2}>0$ :

nenhuma singularidade quando $\lambda<0$,

foco hiperbólico de $X_{\lambda}+$ sela hiperbólica de $F_{Z}$ quando $\lambda>0$,

(v) $k<0, \sigma^{2} \geq 4 \Delta>0$ :

nenhuma singularidade quando $\lambda<0$, nó hiperbólico de $X_{\lambda}+$ sela hiperbólica de $F_{Z}$ quando $\lambda>0$.

(vi) $k<0, \Delta<0$ :

sela hiperbólica de $X_{\lambda}$ quando $\lambda<0$, sela hiperbólica de $F_{Z}$ quando $\lambda>0$.

demonstração

Tem-se $X_{\lambda}(x, y)=(-\Delta y+\lambda, x+\sigma y)$.

O ponto fixo de $X_{\lambda}$ é dado por 
$(-\Delta y+\lambda, x+\sigma y)=(0,0) \Rightarrow(x, y)=(-\sigma \lambda / \Delta, \lambda / \Delta)$

Logo, $\mathrm{DX}_{\lambda}=\left[\begin{array}{cc}0 & -\Delta \\ 1 & \sigma\end{array}\right]$ e $\operatorname{det}\left(\mathrm{DX}_{\lambda}-\mu \mathrm{I}\right)=\mu^{2}-\sigma \mu+\Delta=0 \Rightarrow$

$\Rightarrow \Delta_{\mu}=\sigma^{2}-4 \Delta$

Se $\Delta_{\mu} \geq 0$, então $\sigma^{2} \geq 4 \Delta$ e a singularidade de $X_{\lambda}$ é uma sela ou um nó.

Se $\Delta_{\mu}<0$, então $\sigma^{2}<4 \Delta$ e a singularidade de $X_{\lambda}$ é um foco.

Analisando-se cada caso, tem-se

Se $\sigma^{2}-4 \Delta=0$, então $\mu=\sigma / 2$ é auto-valor duplo da singularidade de $X_{\lambda}$, que portanto é um nó. Se $\mu=\sigma / 2 \neq 0$, o nó é hiperbólico.

Se $\sigma^{2}-4 \Delta>0$, então $\mu=\frac{\sigma \pm \sqrt{ }\left(\sigma^{2}-4 \Delta\right)}{2}$ e a singularidade é sela ou nó.

Para que a singularidade seja um nó hiperbólico, os números $\sigma+\sqrt{ }\left(\sigma^{2}-4 \Delta\right)$ e $\sigma-\sqrt{ }\left(\sigma^{2}-4 \Delta\right)$ devem ter o mesmo sinal, ou seja,

$\sigma+\sqrt{ }\left(\sigma^{2}-4 \Delta\right)>0$ e $\sigma-\sqrt{ }\left(\sigma^{2}-4 \Delta\right)>0$
ou
$\sigma+\sqrt{ }\left(\sigma^{2}-4 \Delta\right)<0$ e $\sigma-\sqrt{ }\left(\sigma^{2}-4 \Delta\right)<0$

De (1), tem-se

$\sigma>-\sqrt{ }\left(\sigma^{2}-4 \Delta\right)$ e $\sigma>\sqrt{ }\left(\sigma^{2}-4 \Delta\right) \Rightarrow \sigma^{2}>\sigma^{2}-4 \Delta \Rightarrow 4 \Delta>0 \Rightarrow \Delta>0$.

De (2), tem-se

$\sigma<-\sqrt{ }\left(\sigma^{2}-4 \Delta\right)$ e $\sigma<\sqrt{ }\left(\sigma^{2}-4 \Delta\right) \Rightarrow \sigma^{2}>\sigma^{2}-4 \Delta \Rightarrow 4 \Delta>0 \Rightarrow \Delta>0$

Ou seja, para que a singularidade $(x, y)=(-\sigma \lambda / \Delta, \lambda / \Delta)$ seja um nó hiperbólico, deve-se ter $\sigma^{2}-4 \Delta>0$ e $\Delta>0$. Este nó será atrator caso $\sigma<0$ e repulsor caso $\sigma>0$.

Para que a singularidade seja uma sela hiperbólica, $\sigma+\sqrt{ }\left(\sigma^{2}-4 \Delta\right)$ e $\sigma-\sqrt{ }\left(\sigma^{2}-4 \Delta\right)$ devem ter sinais contrários, ou seja,

$$
\sigma+\sqrt{ }\left(\sigma^{2}-4 \Delta\right)>0 \text { e } \sigma-\sqrt{ }\left(\sigma^{2}-4 \Delta\right)<0
$$

De (3), tem-se 


$$
\begin{aligned}
& \sigma>-\sqrt{ }\left(\sigma^{2}-4 \Delta\right) \text { e } \sigma<\sqrt{ }\left(\sigma^{2}-4 \Delta\right) \Rightarrow-\sqrt{ }\left(\sigma^{2}-4 \Delta\right)<\sigma<\sqrt{ }\left(\sigma^{2}-4 \Delta\right) \Rightarrow \\
& \Rightarrow|\sigma|<\sqrt{ }\left(\sigma^{2}-4 \Delta\right) \Rightarrow \sigma^{2}<\sigma^{2}-4 \Delta \Rightarrow 4 \Delta<0 \Rightarrow \Delta<0 .
\end{aligned}
$$

Logo, para que a singularidade $(x, y)=(-\sigma \lambda / \Delta, \lambda / \Delta)$ seja uma sela hiperbólica, deve-se ter $\sigma^{2}-4 \Delta>0$ e $\Delta<0$.

Neste caso, os auto-valores e auto-vetores associados à sela são $\mu_{1}=\left(\sigma+\sqrt{ }\left(\sigma^{2}-4 \Delta\right)\right) / 2, \mu_{2}=\left(\sigma-\sqrt{ }\left(\sigma^{2}-4 \Delta\right)\right) / 2, \mathrm{v}_{1}=\left(\left(\sigma-\sqrt{ }\left(\sigma^{2}-4 \Delta\right)\right) / 2,-1\right) \mathrm{e}$ $\mathrm{v}_{2}=\left(\left(\sigma+\sqrt{ }\left(\sigma^{2}-4 \Delta\right)\right) / 2,-1\right)$.

Se $\Delta_{\mu}<0$, então $\sigma^{2}<4 \Delta$ e a singularidade de $X_{\lambda}$ é um foco. Para que seja um foco hiperbólico, a parte real dos auto-valores deve ser diferente de zero, ou seja, $\sigma \neq 0$. Logo, a singularidade $(x, y)=(-\sigma \lambda / \Delta, \lambda / \Delta)$ de $X_{\lambda}$ é um foco hiperbólico se $0<\sigma^{2}<4 \Delta$. Este foco é atrator caso $\sigma<0$ e repulsor caso $\sigma>0$.

Levando-se em conta que o campo descontínuo $Z_{\lambda}$ é dado por $X_{\lambda}$ se $f(\mathrm{x}, \mathrm{y})=\mathrm{y}>0$, a singularidade de $\mathrm{X}_{\lambda}$ será uma singularidade de $Z_{\lambda}$ se $\mathrm{y}=\lambda / \Delta>0$, ou seja, se $\lambda$ e $\Delta$ tiverem o mesmo sinal.

Agora procurando-se as singularidades de $\mathrm{F}_{\mathrm{Z}}$.

Tem-se $\mathrm{X}_{\lambda} f=\mathrm{x}+\sigma \mathrm{y}$ e $\mathrm{Y} f=1>0$.

$\mathrm{Em} \mathrm{D}=\{\mathrm{y}=0\}, \mathrm{X}_{\lambda} f . \mathrm{Y}_{\lambda} f=\mathrm{x}, \log 0$, se $\mathrm{x}>0$ o ponto $(\mathrm{x}, 0)$ pertence ao arco de costura, e se $x<0$ o ponto $(x, 0)$ pertence ao arco de deslizamento.

A singularidade de Filippov, se existir, pertence ao arco deslizante, portanto deve ter $x<0$. Além disto, devem valer $\operatorname{det}\left(X_{\lambda}, Y_{\lambda}\right)(x, 0)=0$ e $\left.\mathrm{d}\left(\operatorname{det}\left(\mathrm{X}_{\lambda}, \mathrm{Y}_{\lambda}\right)\right)\right|_{\mathrm{D}} \neq 0$.

$$
\begin{aligned}
& \text { Como } \operatorname{det}\left(X_{\lambda}, Y_{\lambda}\right)=(-\Delta y+\lambda) \cdot 1-(x+\sigma y) k \\
& \text { tem-se } \operatorname{det}\left(X_{\lambda}, Y\right)(x, 0)=\lambda-x k=0 \Rightarrow x=\lambda / k \\
& \text { e }\left.d\left(\operatorname{det}\left(X_{\lambda}, Y\right)\right)\right|_{D}=\frac{\partial}{\partial x}\left(\operatorname{det}\left(X_{\lambda}, Y\right)\right)=-k
\end{aligned}
$$

Logo, $F_{z}$ tem uma singularidade hiperbólica em $(x, y)=(\lambda / k, 0)$ se $\mathrm{k} \neq 0$ e $\lambda / \mathrm{k}<0$. Esta singularidade será um nó quando $\mathrm{k}>0$ e uma sela quando $\mathrm{k}<0$. 

do Lema.

Combinando-se as várias possibilidades, obtém-se a lista do enunciado

As figuras abaixo representam os seis casos do Lema, para $\lambda>0$ e $\lambda<0$. Em todos os casos foi considerado que $\sigma>0$. A reta horizontal representa a curva $\mathrm{D}$, que coincide com o eixo $\mathrm{x}$ e a reta vertical representa o eixo y.

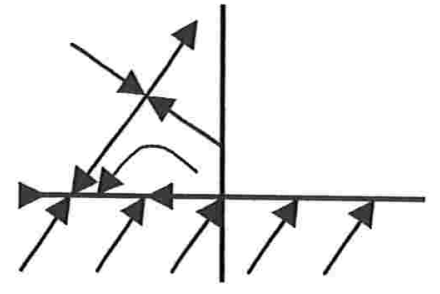

Caso (i): $\lambda<0$

Fig. 69

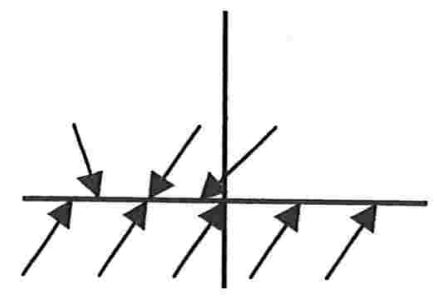

Caso (ii): $\lambda<0$

Fig. 71

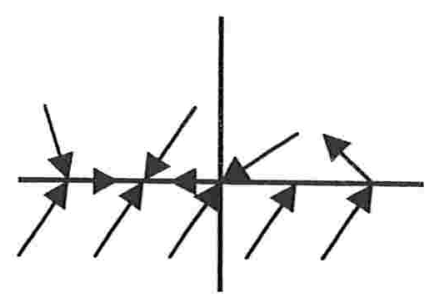

Caso (iii): $\lambda<0$

Fig. 73

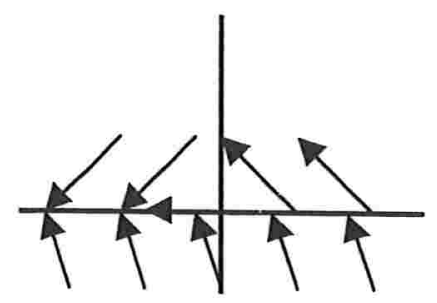

Caso (iv): $\lambda<0$

Fig. 75

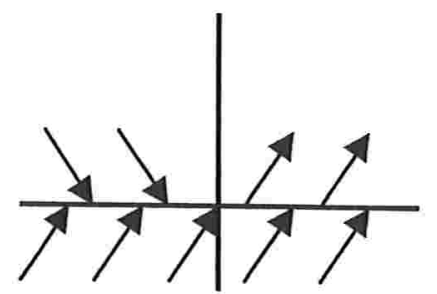

Caso (i): $\lambda>0$

Fig. 70

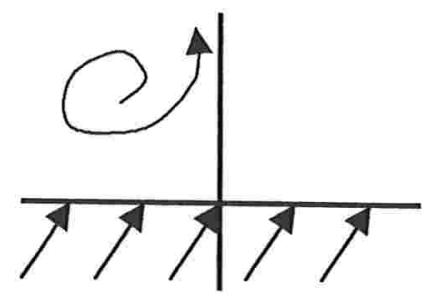

Caso (ii): $\lambda>0$

Fig. 72

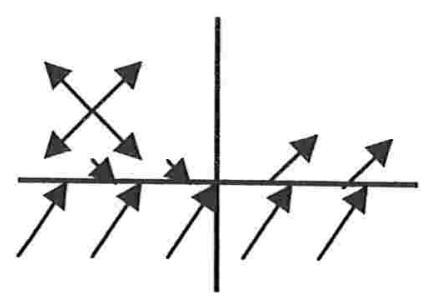

Caso (iii): $\lambda>0$

Fig. 74

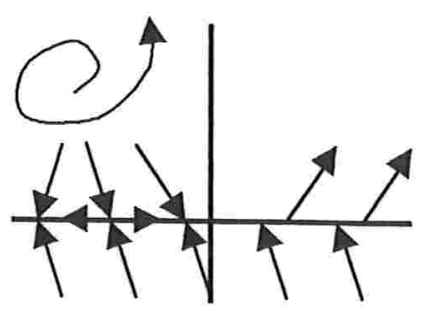

Caso (iv): $\lambda>0$

Fig. 76 


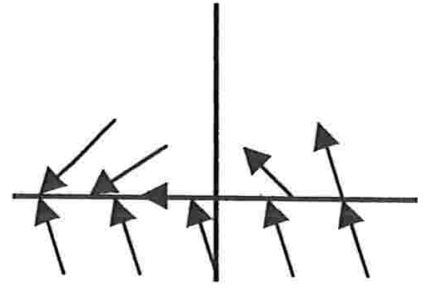

Caso (v): $\lambda<0$

Fig. 77

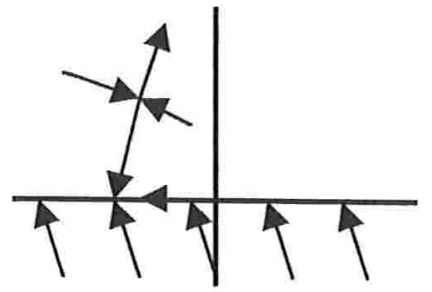

Caso (vi): $\lambda<0$

Fig. 79

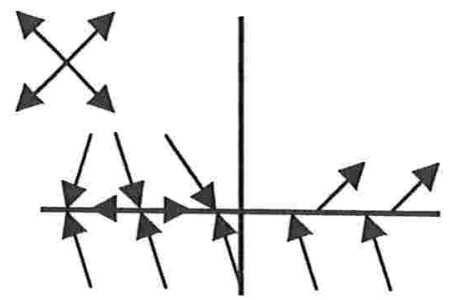

Caso (v): $\lambda>0$

Fig. 78

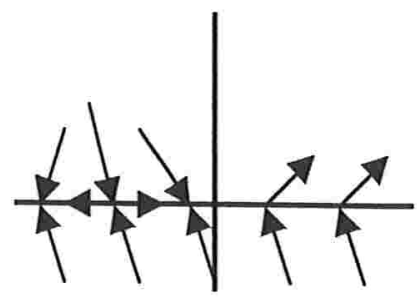

Caso (vi): $\lambda>0$

Fig. 80

\subsection{Observações}

Os seguintes cálculos serão úteis para as proposições posteriores:

Dada uma função de transição $\varphi$, a regularização da família $Z_{\lambda}=\left(X_{\lambda}, Y_{\lambda}\right)$ é dada por

$$
\begin{aligned}
& Z_{\varepsilon, \lambda}(\mathrm{x}, \mathrm{y})=\left(1-\varphi_{\varepsilon}(\mathrm{y})\right) Y_{\lambda}(\mathrm{x}, \mathrm{y})+\varphi_{\varepsilon} \mathrm{X}_{\lambda}(\mathrm{x}, \mathrm{y})= \\
& =\left(1-\varphi_{\varepsilon}(\mathrm{y})\right)(\mathrm{k}, 1)+\varphi_{\varepsilon}(\mathrm{y})(-\Delta \mathrm{y}+\lambda, \mathrm{x}+\sigma \mathrm{y})= \\
& =\left(\mathrm{k}-\varphi_{\varepsilon}(\mathrm{y}) \mathrm{k}-\varphi_{\varepsilon}(\mathrm{y}) \Delta \mathrm{y}+\varphi_{\varepsilon}(\mathrm{y}) \lambda, 1-\varphi_{\varepsilon}(\mathrm{y})+\varphi_{\varepsilon}(\mathrm{y}) \mathrm{x}+\varphi_{\varepsilon}(\mathrm{y}) \sigma \mathrm{y}\right)= \\
& =\left(-\varphi_{\varepsilon}(\mathrm{y})(\mathrm{k}+\Delta \mathrm{y}-\lambda)+\mathrm{k}, 1-\varphi_{\varepsilon}(\mathrm{y})(1-\mathrm{x}-\sigma \mathrm{y})\right)
\end{aligned}
$$

Logo, uma singularidade do campo regularizado deverá satisfazer:

$$
\begin{aligned}
& \mathrm{Z}_{\varepsilon, \lambda}(\mathrm{x}, \mathrm{y})=\left(-\varphi_{\varepsilon}(\mathrm{y})(\mathrm{k}+\Delta \mathrm{y}-\lambda)+\mathrm{k}, 1-\varphi_{\varepsilon}(\mathrm{y})(1-\mathrm{x}-\sigma \mathrm{y})\right)=(0,0) \\
& \Rightarrow \varphi_{\varepsilon}(\mathrm{y})=\mathrm{k} /(\mathrm{k}+\Delta \mathrm{y}-\lambda) \\
& \varphi_{8}(\mathrm{y})=1 /(1-\mathrm{x}-\sigma \mathrm{y}) \quad \text { (B). }
\end{aligned}
$$

Nota-se que quando a expressão (A) tiver solução, o valor de y poderá ser obtido implicitamente desta expressão e então o valor de $x$ poderá ser obtido da expressão (B). 
Calculando-se a matriz $\mathrm{DZ}_{\varepsilon, \lambda}$ para possibilitar o estudo destas singularidades:

$\mathrm{D} Z_{\varepsilon, \lambda}=\left[\begin{array}{cl}0 & -\left[\varphi_{\varepsilon}(\mathrm{y})\right]^{\prime}(\mathrm{k}+\Delta \mathrm{y}-\lambda)-\Delta \varphi_{\varepsilon}(\mathrm{y}) \\ \varphi_{\mathrm{\varepsilon}}(\mathrm{y}) & -\left[\varphi_{\varepsilon}(\mathrm{y})\right]^{\prime}(1-\mathrm{x}-\sigma \mathrm{y})+\sigma \varphi_{\varepsilon}(\mathrm{y})\end{array}\right]$

Logo, o polinômio característico é dado por

$$
\begin{aligned}
& \left|\mathrm{DZ} Z_{\varepsilon, \lambda}-\mu \mathrm{I}\right|=-\mu\left(-\left[\varphi_{\varepsilon}(\mathrm{y})\right]^{\prime}(1-\mathrm{x}-\sigma \mathrm{y})+\sigma \varphi_{\varepsilon}(\mathrm{y})-\mu\right)-\varphi_{\varepsilon}(\mathrm{y})\left(-\left[\varphi_{\varepsilon}(\mathrm{y})\right]^{\prime}(\mathrm{k}+\Delta \mathrm{y}-\lambda)-\right. \\
& \left.\Delta \varphi_{\varepsilon}(\mathrm{y})\right)=0 \\
& \Rightarrow \quad \mu^{2}+\mu\left(\left[\varphi_{\varepsilon}(\mathrm{y})\right]^{\prime}(1-\mathrm{x}-\sigma \mathrm{y})-\sigma \varphi_{\varepsilon}(\mathrm{y})\right)+\varphi_{\varepsilon}(\mathrm{y})\left(\left[\varphi_{\varepsilon}(\mathrm{y})\right]^{\prime}(\mathrm{k}+\Delta \mathrm{y}-\lambda)+\Delta \varphi_{\varepsilon}(\mathrm{y})\right)=0
\end{aligned}
$$

Resolvendo-se a equação de segundo grau, tem-se:

$$
\begin{aligned}
& \Delta_{\mu}=\left(\left[\varphi_{\varepsilon}(\mathrm{y})\right]^{\prime}(1-\mathrm{x}-\sigma \mathrm{y})-\sigma \varphi_{\varepsilon}(\mathrm{y})\right)^{2}-4 \varphi_{\varepsilon}(\mathrm{y})\left(\left[\varphi_{\varepsilon}(\mathrm{y})\right]^{\prime}(\mathrm{k}+\Delta \mathrm{y}-\lambda)+\Delta \varphi_{\varepsilon}(\mathrm{y})\right) \\
& =\left(\left[\varphi_{\varepsilon}(y)\right]^{\prime}\right)^{2}(1-x-\sigma y)^{2}-2\left[\varphi_{\varepsilon}(y)\right]^{\prime}(1-x-\sigma y) \sigma \varphi_{\varepsilon}(y)+\sigma^{2}\left(\varphi_{\varepsilon}(y)\right)^{2}- \\
& 4 \varphi_{\varepsilon}(\mathrm{y})\left[\varphi_{\varepsilon}(\mathrm{y})\right]^{\prime}(\mathrm{k}+\Delta \mathrm{y}-\lambda)-4 \Delta\left(\varphi_{\varepsilon}(\mathrm{y})\right)^{2} \\
& \text { Usando-se (A) e (B), tem-se } \\
& \mu^{2}+\mu\left(\left[\varphi_{\varepsilon}(\mathrm{y})\right]^{\prime} / \varphi_{\varepsilon}(\mathrm{y})-\sigma \varphi_{\varepsilon}(\mathrm{y})\right)+\varphi_{\varepsilon}(\mathrm{y})\left(\left[\varphi_{\varepsilon}(\mathrm{y})\right]^{\prime}\left(\mathrm{k} / \varphi_{\varepsilon}(\mathrm{y})\right)+\Delta \varphi_{\varepsilon}(\mathrm{y})\right)=0 \\
& \text { e } \\
& \Delta_{\mu}=\left(\left[\varphi_{\varepsilon}(\mathrm{y})\right]^{\prime}\right)^{2}\left(1 / \varphi_{\varepsilon}(\mathrm{y})\right)^{2}-2\left[\varphi_{\varepsilon}(\mathrm{y})\right]^{\prime}\left(1 / \varphi_{\varepsilon}(\mathrm{y})\right) \sigma \varphi_{\varepsilon}(\mathrm{y})+\sigma^{2}\left(\varphi_{\varepsilon}(\mathrm{y})\right)^{2}- \\
& 4 \varphi_{\varepsilon}(\mathrm{y})\left[\varphi_{\varepsilon}(\mathrm{y})\right]^{\prime}\left(\mathrm{k} / \varphi_{\varepsilon}(\mathrm{y})\right)-4 \Delta\left(\varphi_{\varepsilon}(\mathrm{y})\right)^{2}= \\
& =\left(\left[\varphi_{\varepsilon}(\mathrm{y})\right]^{\prime} / \varphi_{\varepsilon}(\mathrm{y})\right)^{2}-2\left[\varphi_{\varepsilon}(\mathrm{y})\right]^{\prime} \sigma+\sigma^{2}\left(\varphi_{\varepsilon}(\mathrm{y})\right)^{2}-4\left[\varphi_{\varepsilon}(\mathrm{y})\right]^{\prime} \mathrm{k}-4 \Delta\left(\varphi_{\varepsilon}(\mathrm{y})\right)^{2}= \\
& =\left(\left[\varphi_{\varepsilon}(\mathrm{y})\right]^{\prime} / \varphi_{\varepsilon}(\mathrm{y})\right)^{2}+\left[\varphi_{\varepsilon}(\mathrm{y})\right]^{\prime}(-2 \sigma-4 \mathrm{k})+\left(\varphi_{\varepsilon}(\mathrm{y})\right)^{2}\left(\sigma^{2}-4 \Delta\right) \\
& \mu=1 / 2\left(-\left(\left[\varphi_{\varepsilon}(\mathrm{y})\right]^{\prime}(1-\mathrm{x}-\sigma \mathrm{y})-\sigma \varphi_{\varepsilon}(\mathrm{y})\right) \pm \sqrt{ } \Delta_{\mu}\right)= \\
& =1 / 2\left(-\left[\varphi_{\varepsilon}(\mathrm{y})\right]^{\prime} / \varphi_{\varepsilon}(\mathrm{y})+\sigma \varphi_{\varepsilon}(\mathrm{y}) \pm \sqrt{ } \Delta_{\mu}\right) .
\end{aligned}
$$

Nas proposições seguintes serão feitas a regularização em cada um dos casos do Lema 1.2. Nota-se que não há uma majoração do parâmetro $\varepsilon$. Também é interessante que seja notado que nos casos (iii), para $\sigma<0$, e (vi) não se observa um fenômeno de bifurcação da família $Z_{\varepsilon, \lambda}$. 


\subsection{Proposição}

Seja $Z_{\lambda}$ uma familia de campos vetoriais descontínuos com $k>0, \Delta<0$ (caso i) e $\Delta / k+\sigma \neq 0$. Dada uma função de transição $\varphi$, a família de campos vetoriais regularizados $Z_{\varepsilon, \lambda}$ possui valores dos parâmetros $\left(\varepsilon, \lambda_{0}(\varepsilon)\right)$, onde $\varepsilon>0$, para os quais $Z_{\varepsilon, \lambda o}$ possui uma singularidade tipo sela-nó. Para $\lambda>\lambda_{0}$ os campos vetoriais regularizados $Z_{\varepsilon, \lambda}$ não possuem singularidades e para $\lambda<\lambda_{0}$, os campos vetoriais regularizados $Z_{\varepsilon, \lambda}$ possuem duas singularidades, uma sela e um nó, ambos hiperbólicos.

demonstração

O primeiro passo é estudar as expressões (A) e (B) e encontrar o(s) $\operatorname{par}(\mathrm{es})(\mathrm{x}, \mathrm{y})$ que as satisfazem, e em que condições isto acontece.

Tem-se

$$
\begin{aligned}
& \varphi_{\varepsilon}(\mathrm{y})=\mathrm{k} /(\mathrm{k}+\Delta \mathrm{y}-\lambda) \\
& \mathrm{e} \\
& \varphi_{\varepsilon}(\mathrm{y})=1 /(1-\mathrm{x}-\sigma \mathrm{y})
\end{aligned}
$$

Seja $g_{\lambda}(y)=k /(k+\Delta y-\lambda)$. Se existir y tal que $\varphi_{\varepsilon}(y)=g_{\lambda}(y)$, então este valor y será a ordenada de uma singularidade do campo $Z_{\varepsilon, \lambda}$. abaixo:

Os gráficos de $\mathrm{g}_{0}(\mathrm{y})=\mathrm{k} /(\mathrm{k}+\Delta \mathrm{y})$ e de $\varphi_{\varepsilon}(\mathrm{y})$ são dados na figura

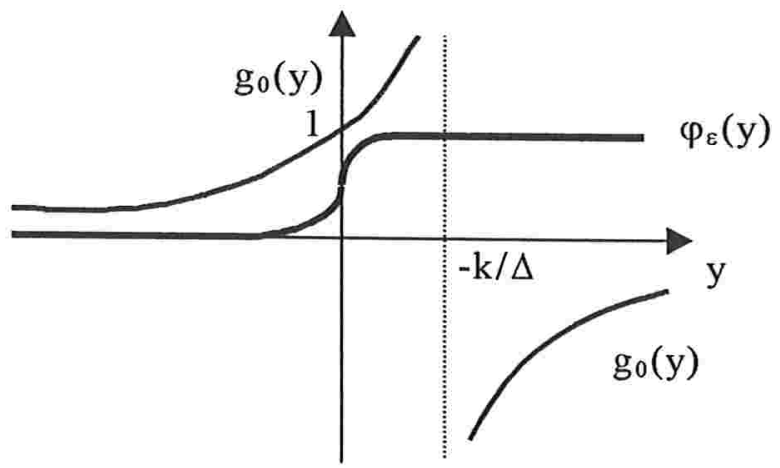

Fig. 81

Quando $\lambda$ cresce, a assíntota do gráfico de $g_{\lambda}(y)$ se desloca para a esquerda, logo o gráfico de $g_{\lambda}(y)$ não cruzará o gráfico de $\varphi_{\varepsilon}(y)$. Quando $\lambda$ decresce, a assíntota do gráfico de $\mathrm{g}_{\lambda}(\mathrm{y})$. se desloca para a direita, logo, mantendo-se $\varepsilon$ fixo, existe um único valor $\lambda_{0}=\lambda_{0}(\varepsilon)<0$ para o qual os gráficos de $g_{\lambda}(y)$ e $\varphi_{\varepsilon}(y)$ se tangenciam num único ponto $\left(\mathrm{y}_{0}, \mathrm{~g}_{\lambda_{0}}\left(\mathrm{y}_{0}\right)\right)=$ $\left(\mathrm{y}_{0}, \varphi_{\varepsilon}\left(\mathrm{y}_{0}\right)\right)$, como ilustra a figura a seguir. 


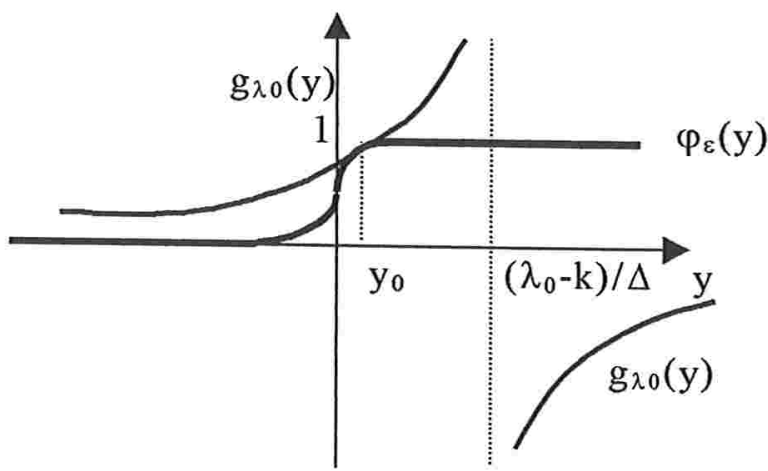

Fig. 82

Para $\lambda<\lambda_{0}$ os gráficos de $g_{\lambda}(\mathrm{y})$ e $\varphi_{\varepsilon}(\mathrm{y})$ se cruzarão duas vezes, nos pontos $\left(\mathrm{y}_{1}, \mathrm{~g}_{\lambda}\left(\mathrm{y}_{1}\right)\right)=\left(\mathrm{y}_{1}, \varphi_{\varepsilon}\left(\mathrm{y}_{1}\right)\right)$ e $\left(\mathrm{y}_{2}, \mathrm{~g}_{\lambda}\left(\mathrm{y}_{2}\right)\right)=\left(\mathrm{y}_{2}, \varphi_{\varepsilon}\left(\mathrm{y}_{2}\right)\right)$, como ilustra a figura abaixo:

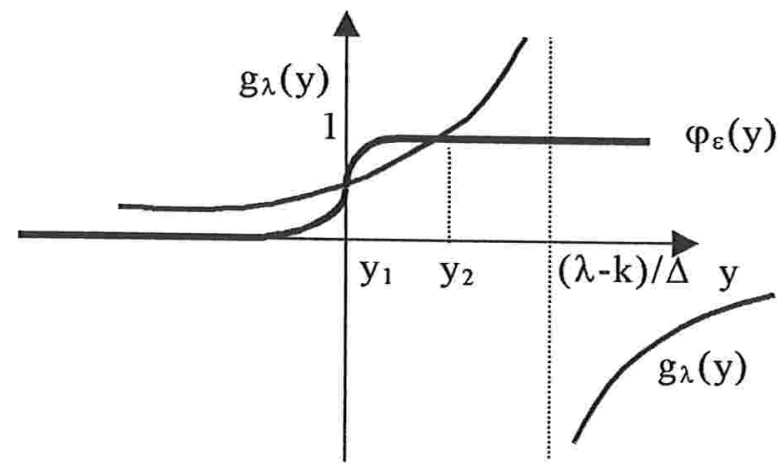

Fig. 83

Seja $\left(\mathrm{x}_{0}, \mathrm{y}_{0}\right)$ a solução das equações $(\mathrm{A})$ e $(\mathrm{B})$, onde $\left(\mathrm{y}_{0}, \mathrm{~g}_{\lambda_{0}}\left(\mathrm{y}_{0}\right)\right)=$ $\left(\mathrm{y}_{0}, \varphi_{\varepsilon}\left(\mathrm{y}_{0}\right)\right)$ é o ponto de tangência entre os gráficos de $g_{\lambda_{0}}$ e $\varphi_{\varepsilon}$, ou seja, $\left(\mathrm{x}_{0}, \mathrm{y}_{0}\right)$ é a única singularidade de $Z_{\varepsilon, \lambda 0}$.

Afirma-se que $\left(\mathrm{x}_{0}, \mathrm{y}_{0}\right)$ é uma sela-nó de $Z_{\varepsilon, \lambda 0}$.

De fato, como os gráficos de $g_{\lambda}(y)$ e $\varphi_{\varepsilon}(y)$ se tangenciam para $y=y_{0}$, então $\left(\mathrm{g}_{\lambda 0}\left(\mathrm{y}_{0}\right)\right)^{\prime}=\left(\varphi_{\varepsilon}\left(\mathrm{y}_{0}\right)\right)^{\prime}$, isto é,

$$
\left(\varphi_{\varepsilon}\left(\mathrm{y}_{0}\right)\right)^{\prime}=-\Delta \mathrm{k} /\left(\mathrm{k}+\Delta \mathrm{y}_{0}-\lambda\right)^{2}=-\Delta\left(\varphi_{\varepsilon}\left(\mathrm{y}_{0}\right)\right)^{2} / \mathrm{k} .
$$

Logo os auto-valores desta singularidade são

$$
\mu=1 / 2\left(-\left[\varphi_{\varepsilon}\left(\mathrm{y}_{0}\right)\right]^{\prime} / \varphi_{\varepsilon}\left(\mathrm{y}_{0}\right)+\sigma \varphi_{\varepsilon}\left(\mathrm{y}_{0}\right) \pm \sqrt{ } \Delta_{\mu}\right)
$$


Onde $\Delta_{\mu}=\left(\left[\varphi_{\varepsilon}\left(\mathrm{y}_{0}\right)\right]^{\prime} / \varphi_{\mathrm{s}}\left(\mathrm{y}_{0}\right)\right)^{2}+\left[\varphi_{\varepsilon}\left(\mathrm{y}_{0}\right)\right]^{\prime}(-2 \sigma-4 \mathrm{k})+\left(\varphi_{\varepsilon}\left(\mathrm{y}_{0}\right)\right)^{2}\left(\sigma^{2}-4 \Delta\right)$.

Usando-se (C) tem-se

$$
\begin{aligned}
& \Delta_{\mu}=\left(-\Delta\left(\varphi_{\varepsilon}\left(\mathrm{y}_{0}\right)\right)^{2} / \mathrm{k} \varphi_{\varepsilon}\left(\mathrm{y}_{0}\right)\right)^{2}-\Delta\left(\varphi_{\varepsilon}\left(\mathrm{y}_{0}\right)\right)^{2}(-2 \sigma-4 \mathrm{k}) / \mathrm{k}+\left(\varphi_{\varepsilon}\left(\mathrm{y}_{0}\right)\right)^{2}\left(\sigma^{2}-4 \Delta\right) \\
& =\Delta^{2}\left(\varphi_{\varepsilon}\left(\mathrm{y}_{0}\right)\right)^{2} / \mathrm{k}^{2}+\left(\varphi_{\varepsilon}\left(\mathrm{y}_{0}\right)\right)^{2}\left(2 \Delta \sigma / \mathrm{k}+4 \Delta+\sigma^{2}-4 \Delta\right)= \\
& =\Delta^{2}\left(\varphi_{\varepsilon}\left(\mathrm{y}_{0}\right)\right)^{2} / \mathrm{k}^{2}+\left(\varphi_{\varepsilon}\left(\mathrm{y}_{0}\right)\right)^{2}\left(2 \Delta \sigma / \mathrm{k}+\sigma^{2}\right)= \\
& =\left(\varphi_{\varepsilon}\left(\mathrm{y}_{0}\right)\right)^{2}\left(\Delta^{2} / \mathrm{k}^{2}+2 \Delta \sigma / \mathrm{k}+\sigma^{2}\right)=\left(\varphi_{\varepsilon}\left(\mathrm{y}_{0}\right)\right)^{2}(\Delta / \mathrm{k}+\sigma)^{2}>0 \\
& \mu=1 / 2\left(\Delta\left(\varphi_{\varepsilon}\left(\mathrm{y}_{0}\right)\right)^{2} / \mathrm{k} \varphi_{\varepsilon}\left(\mathrm{y}_{0}\right)+\sigma \varphi_{\varepsilon}\left(\mathrm{y}_{0}\right) \pm \sqrt{ } \Delta_{\mu}\right) \\
& =1 / 2\left(\Delta \varphi_{\varepsilon}\left(\mathrm{y}_{0}\right) / \mathrm{k}+\sigma \varphi_{\varepsilon}\left(\mathrm{y}_{0}\right) \pm \sqrt{ }\left(\left(\varphi_{\varepsilon}\left(\mathrm{y}_{0}\right)\right)^{2}(\Delta / \mathrm{k}+\sigma)^{2}\right)\right)= \\
& =1 / 2\left(\varphi_{\varepsilon}\left(\mathrm{y}_{0}\right)(\Delta / \mathrm{k}+\sigma) \pm \varphi_{\varepsilon}\left(\mathrm{y}_{0}\right) \sqrt{ }(\Delta / \mathrm{k}+\sigma)^{2}\right)= \\
& =1 / 2 \varphi_{\varepsilon}\left(\mathrm{y}_{0}\right)((\Delta / \mathrm{k}+\sigma) \pm|\Delta / \mathrm{k}+\sigma|) \\
& \Rightarrow \text { os auto-valores são } \mu_{0}=0 \text { e } \mu_{1}=\varphi_{\varepsilon}\left(\mathrm{y}_{0}\right)(\Delta / \mathrm{k}+\sigma) .
\end{aligned}
$$

Portanto, $\left(\mathrm{x}_{0}, \mathrm{y}_{0}\right)$ é uma sela-nó de $Z_{\varepsilon \lambda 0}$.

Afirma-se que se $\lambda<\lambda_{0}, Z_{\varepsilon, \lambda}$ tem dois pontos singulares, um sendo sela e o outro um nó.

De fato, se $\lambda<\lambda_{0}$, como pode ser observado na figura 83 , os gráficos de $g_{\lambda}(y)$ e $\varphi_{\varepsilon}(y)$ se cruzam duas vezes, nos pontos $\left(\mathrm{y}_{1}, \mathrm{~g}_{\lambda}\left(\mathrm{y}_{1}\right)\right)=\left(\mathrm{y}_{1}, \varphi_{\varepsilon}\left(\mathrm{y}_{1}\right)\right)$ e $\left(y_{2}, g_{\lambda}\left(y_{2}\right)\right)=\left(y_{2}, \varphi_{\varepsilon}\left(y_{2}\right)\right)$. Através da equação (B) encontra-se os valores $\mathrm{x}_{1}$ e $\mathrm{x}_{2}$ correspondentes a $\mathrm{y}_{1}$ e $\mathrm{y}_{2}$, isto é, tais que os pares $\left(\mathrm{x}_{1}, \mathrm{y}_{1}\right)$ e $\left(\mathrm{x}_{2}, \mathrm{y}_{2}\right)$ são as únicas soluções de (A) e (B), e portanto são singularidades de $Z_{\varepsilon, \lambda}$. Resta agora investigar a natureza destas singularidades.

$$
\begin{aligned}
& \text { Observa-se que } \\
& \left(\varphi_{\varepsilon}\left(\mathrm{y}_{1}\right)\right)^{\prime}>\left(\mathrm{g}_{\lambda}\left(\mathrm{y}_{1}\right)\right)^{\prime}=-\Delta \mathrm{k} /\left(\mathrm{k}+\Delta \mathrm{y}_{1}-\lambda\right)^{2}=-\Delta\left(\varphi_{\varepsilon}\left(\mathrm{y}_{1}\right)\right)^{2} / \mathrm{k} \\
& \mathrm{e} \\
& \left(\varphi_{\varepsilon}\left(\mathrm{y}_{2}\right)\right)^{\prime}<\left(\mathrm{g}_{\lambda}\left(\mathrm{y}_{2}\right)\right)^{\prime}=-\Delta \mathrm{k} /\left(\mathrm{k}+\Delta \mathrm{y}_{2}-\lambda\right)^{2}=-\Delta\left(\varphi_{\varepsilon}\left(\mathrm{y}_{2}\right)\right)^{2} / \mathrm{k} .
\end{aligned}
$$

Observa-se que para uma singularidade com auto-valores dados por $\mu=1 / 2\left(-b_{\mu} \pm \sqrt{ } \Delta_{\mu}\right)$ ser uma sela hiperbólica deve-se ter

$$
\left.\begin{array}{l}
-\mathrm{b}_{\mu}+\sqrt{ } \Delta_{\mu}>0 \Rightarrow \sqrt{ } \Delta_{\mu}>\mathrm{b}_{\mu} \\
-\mathrm{b}_{\mu}-\sqrt{ } \Delta_{\mu}<0 \Rightarrow \sqrt{ } \Delta_{\mu}>-\mathrm{b}_{\mu}
\end{array}\right\} \quad \Rightarrow \sqrt{ } \Delta_{\mu}>\left|\mathrm{b}_{\mu}\right| \Rightarrow \Delta_{\mu}>\mathrm{b}_{\mu}{ }^{2} .
$$


E para esta singularidade ser um nó hiperbólico deve-se ter

$$
\left(-\mathrm{b}_{\mu}+\sqrt{ } \Delta_{\mu}\right)\left(-\mathrm{b}_{\mu}-\sqrt{ }_{\Delta_{\mu}}\right)>0 \Rightarrow \mathrm{b}_{\mu}{ }^{2}-\Delta_{\mu}>0 \Rightarrow 0 \leq \Delta_{\mu}<\mathrm{b}_{\mu}{ }^{2} .
$$

Impondo-se $\Delta_{\mu}<\mathrm{b}_{\mu}{ }^{2}$ tem-se

$$
\begin{aligned}
& \Delta_{\mu}=\left(\left[\varphi_{\varepsilon}(\mathrm{y})\right]^{\prime} / \varphi_{\varepsilon}(\mathrm{y})\right)^{2}+\left[\varphi_{\varepsilon}(\mathrm{y})\right]^{\prime}(-2 \sigma-4 \mathrm{k})+\left(\varphi_{\varepsilon}(\mathrm{y})\right)^{2}\left(\sigma^{2}-4 \Delta\right)< \\
& <\left(-\left[\varphi_{\varepsilon}(\mathrm{y})\right]^{\prime} / \varphi_{\varepsilon}(\mathrm{y})+\sigma \varphi_{\varepsilon}(\mathrm{y})\right)^{2}=\mathrm{b}_{\mu}{ }^{2} \\
& \Rightarrow\left(\left[\varphi_{\varepsilon}(\mathrm{y})\right]^{\prime} / \varphi_{\varepsilon}(\mathrm{y})\right)^{2}+\left[\varphi_{\varepsilon}(\mathrm{y})\right]^{\prime}(-2 \sigma-4 \mathrm{k})+\left(\varphi_{\varepsilon}(\mathrm{y})\right)^{2}\left(\sigma^{2}-4 \Delta\right)< \\
& <\left(\left[\varphi_{\varepsilon}(\mathrm{y})\right]^{\prime} / \varphi_{\mathrm{s}}(\mathrm{y})\right)^{2}-2 \sigma\left[\varphi_{\varepsilon}(\mathrm{y})\right]^{\prime}+\sigma^{2}\left(\varphi_{\varepsilon}\left(\varphi_{\varepsilon}(\mathrm{y})\right)^{2}\right. \\
& \Rightarrow\left[\varphi_{\varepsilon}(\mathrm{y})\right]^{\prime}(-4 \mathrm{k})+\left(\varphi_{\varepsilon}(\mathrm{y})\right)^{2}(-4 \Delta)<0 \\
& \Rightarrow\left[\varphi_{\varepsilon}(\mathrm{y})\right]^{\prime} \mathrm{k}+\left(\varphi_{\varepsilon}(\mathrm{y})\right)^{2} \Delta>0 \\
& \Rightarrow\left[\varphi_{\varepsilon}(\mathrm{y})\right]^{\prime}>-\Delta\left(\varphi_{\varepsilon}(\mathrm{y})\right)^{2} / \mathrm{k}
\end{aligned}
$$

Por (D), a desigualdade acima ocorre em $y=y_{1}$ e, verificando-se que $\Delta_{\mu} \geq 0$ em $y_{1}$, pode-se concluir que a singularidade $\left(x_{1}, y_{1}\right)$ é um nó hiperbólico de $Z_{\varepsilon, \lambda}$.

Afirma-se que $\Delta_{\mu} \geq 0$ em $\mathrm{y}_{1}$.

De fato, em $y_{1}$,

$$
\begin{aligned}
& \Delta_{\mu}=\left(\left[\varphi_{\varepsilon}\left(\mathrm{y}_{1}\right)\right]^{\prime} / \varphi_{\varepsilon}\left(\mathrm{y}_{1}\right)\right)^{2}+\left[\varphi_{\varepsilon}\left(\mathrm{y}_{1}\right)\right]^{\prime}(-2 \sigma-4 \mathrm{k})+\left(\varphi_{\varepsilon}\left(\mathrm{y}_{1}\right)\right)^{2}\left(\sigma^{2}-4 \Delta\right)> \\
& \left.\left.>\left(-\Delta\left(\varphi_{\varepsilon}\left(\mathrm{y}_{1}\right)\right)^{2} / \mathrm{k}\right)^{2} / \varphi_{\varepsilon}\left(\mathrm{y}_{1}\right)\right)^{2}-\Delta\left(\varphi_{\varepsilon}\left(\mathrm{y}_{1}\right)\right)^{2} / \mathrm{k}\right)(-2 \sigma-4 \mathrm{k})+\left(\varphi_{\varepsilon}\left(\mathrm{y}_{1}\right)\right)^{2}\left(\sigma^{2}-4 \Delta\right)= \\
& \left.=\Delta^{2}\left(\varphi_{\varepsilon}\left(\mathrm{y}_{1}\right)\right)^{2} / \mathrm{k}^{2}+\Delta\left(\varphi_{\varepsilon}\left(\mathrm{y}_{1}\right)\right)^{2} / \mathrm{k}\right)(2 \sigma+4 \mathrm{k})+\left(\varphi_{\varepsilon}\left(\mathrm{y}_{1}\right)\right)^{2}\left(\sigma^{2}-4 \Delta\right)= \\
& \left.=\left(\varphi_{\varepsilon}\left(\mathrm{y}_{1}\right)\right)^{2}\left(\Delta^{2} / \mathrm{k}^{2}+2 \Delta \sigma / \mathrm{k}+4 \Delta+\sigma^{2}-4 \Delta\right)\right)= \\
& =\left(\varphi_{\varepsilon}\left(\mathrm{y}_{1}\right)\right)^{2}(\Delta / \mathrm{k}+\sigma)^{2}>0 .
\end{aligned}
$$

Analogamente, impondo-se $\Delta_{\mu}>\mathrm{b}_{\mu}{ }^{2}$ tem-se

$\left[\varphi_{\varepsilon}(\mathrm{y})\right]^{\prime}<-\Delta\left(\varphi_{\varepsilon}(\mathrm{y})\right)^{2} / \mathrm{k}$.

Que, por (E), ocorre para $y=y_{2}$, ou seja, a singularidade $\left(x_{2}, y_{2}\right)$ é uma sela hiperbólica de $Z_{\varepsilon, \lambda}$. 
Afirma-se que se $\lambda>\lambda_{0}, Z_{\varepsilon, \lambda}$ não possui pontos singulares.

De fato, a afirmação acima decorre de não haver um cruzamento entre os gráficos de $g_{\lambda}(y)$ e $\varphi_{\varepsilon}(y)$ e consequentemente as equações (A) e (B) não possuírem soluções.

As figuras abaixo ilustram o campo vetorial descontínuo $Z_{\lambda}$ para os $\operatorname{casos} \lambda>0, \lambda<0, \sigma>0$ e $\sigma<0$.

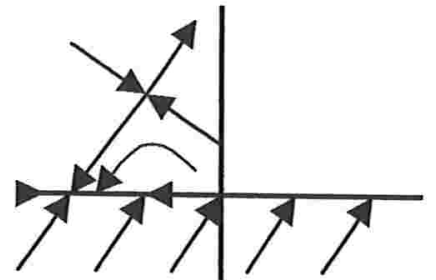

$\lambda<0$ e $\sigma>0$

Fig. 84

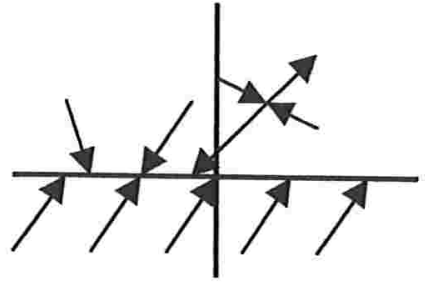

$\lambda<0$ e $\sigma<0$

Fig. 86

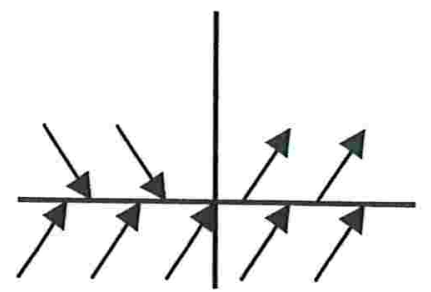

$\lambda>0$ e $\sigma>0$

Fig. 85

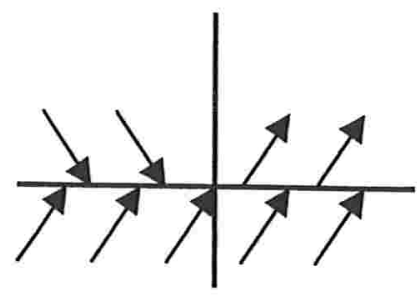

$\lambda>0$ e $\sigma<0$

Fig. 87

\subsection{Proposição}

Seja $Z_{\lambda}$ uma familia de campos vetoriais descontínuos com $k>0 e$ $4 \Delta>\sigma^{2}>0$ (caso ii). Dada uma função de transição $\varphi$, a família de campos vetoriais regularizados $Z_{\varepsilon, \lambda}$ possui valores dos parâmetros $\left(\varepsilon, \lambda_{0}(\varepsilon)\right)$, onde $\varepsilon>0$, para os quais $Z_{\varepsilon, \lambda o}$ possui um nó com auto-valor duplo. Para $\lambda>\lambda_{0}$ os campos vetoriais regularizados $Z_{\varepsilon, \lambda}$ possuem um foco hiperbólico, atrator se $\sigma<0$ e repulsor se $\sigma>0$, e para $\lambda<\lambda_{0}$, os campos vetoriais regularizados $Z_{\varepsilon, \lambda}$ possuem um nó atrator hiperbólico.

demonstração

Analogamente ao que foi feito na Proposição 1.4, deve-se estudar as expressões (A) e (B) e encontrar o(s) par(es) $(x, y)$ que as satisfazem, e em que condições isto acontece. 
Tem-se

$$
\begin{aligned}
& \varphi_{\varepsilon}(\mathrm{y})=\mathrm{k} /(\mathrm{k}+\Delta \mathrm{y}-\lambda) \\
& \mathrm{e} \\
& \varphi_{\varepsilon}(\mathrm{y})=1 /(1-\mathrm{x}-\sigma \mathrm{y})
\end{aligned}
$$

Seja $g_{\lambda}(y)=k /(k+\Delta y-\lambda)$. Se existir y tal que $\varphi_{\varepsilon}(y)=g_{\lambda}(y)$, então este valor y será a ordenada de uma singularidade do campo $Z_{\varepsilon, \lambda}$.

Os gráficos de $g_{\lambda}(y)$ e de $\varphi_{\varepsilon}(y)$ são dados na figura abaixo:

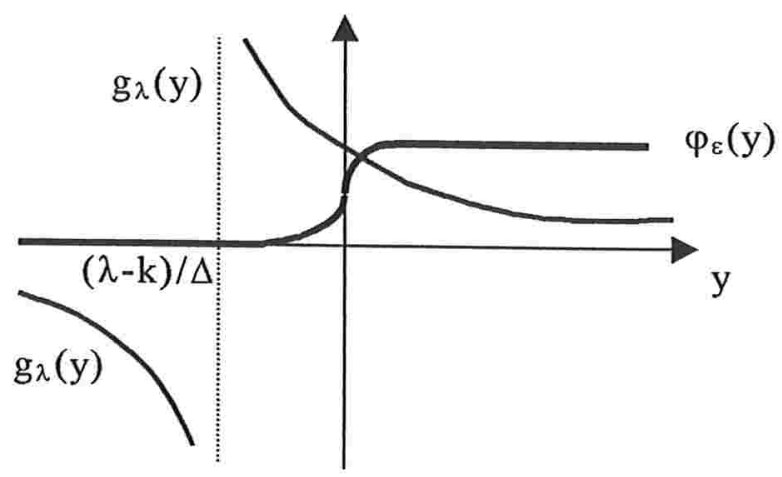

Fig. 88

Quando $\lambda$ cresce, a assíntota do gráfico de $\mathrm{g}_{\lambda}(\mathrm{y})$ se desloca para a direita e quando $\lambda$ decresce, para a esquerda. Para quaisquer valores dos parâmetros $\varepsilon$ e $\lambda$, os dois gráficos se cruzam num único ponto $\left(\mathrm{y}_{\varepsilon}, \mathrm{g}_{\lambda}\left(\mathrm{y}_{\varepsilon}\right)\right)=$ $\left(\mathrm{y}_{\varepsilon \lambda}, \varphi_{\varepsilon}\left(\mathrm{y}_{\varepsilon \lambda}\right)\right)$.

Fixando-se $\varepsilon>0$, para cada $\lambda$, seja $\left(\mathrm{x}_{\varepsilon}, \mathrm{y}_{\varepsilon \lambda}\right)$ a singularidade de $Z_{\varepsilon, \lambda}$, ou seja, a única solução de (A) e (B).

Sabe-se que os auto-valores desta singularidade são

$\mu=1 / 2\left(-\left[\varphi_{\varepsilon}\left(\mathrm{y}_{\mathrm{\varepsilon} \lambda}\right)\right]^{\prime} / \varphi_{\varepsilon}\left(\mathrm{y}_{\mathrm{\varepsilon} \lambda}\right)+\sigma \varphi_{\varepsilon}\left(\mathrm{y}_{\mathrm{\varepsilon} \lambda}\right) \pm \sqrt{ } \Delta_{\mu}\right)$

Onde $\Delta_{\mu}\left(\mathrm{y}_{\varepsilon}\right)=\left(\left[\varphi_{\varepsilon}\left(\mathrm{y}_{\varepsilon \lambda}\right)\right]^{\prime} / \varphi_{\varepsilon}\left(\mathrm{y}_{\varepsilon \lambda} \lambda\right)\right)^{2}+\left[\varphi_{\varepsilon}\left(\mathrm{y}_{\varepsilon} \lambda\right)\right]^{\prime}(-2 \sigma-4 \mathrm{k})+$ $\left(\varphi_{\varepsilon}\left(\mathrm{y}_{\varepsilon \lambda}\right)\right)^{2}\left(\sigma^{2}-4 \Delta\right)$.

Afirma-se que existem valores de $\lambda$ tais que $\Delta_{\mu}\left(\mathrm{y}_{\varepsilon \lambda}\right)<0$ e outros valores de $\lambda$ tais que $\Delta_{\mu}\left(y_{\varepsilon} \lambda\right)>0$.

De fato, seja $\lambda$ tal que a assíntota do gráfico de $g_{\lambda}$ seja a reta $\mathrm{y}=\varepsilon$, como ilustrado na figura abaixo: 


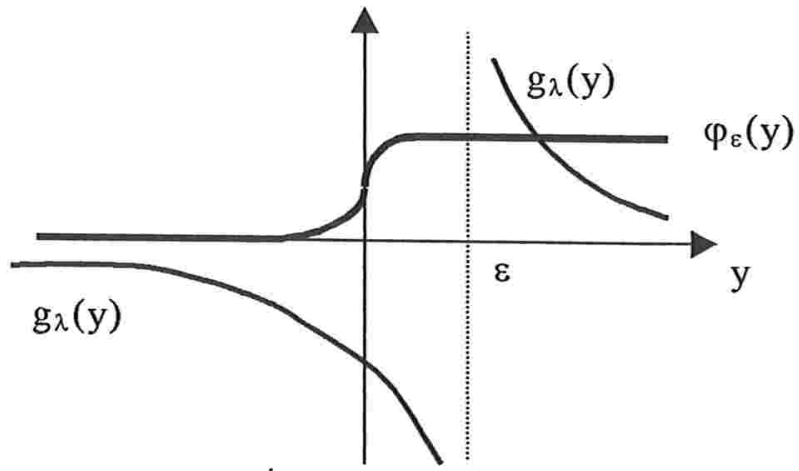

Fig. 89

Ou seja, $(\lambda-\mathrm{k}) / \Delta=\varepsilon \Rightarrow \lambda=\varepsilon \Delta+\mathrm{k}>0$.

Para este valor de $\lambda$, pode-se observar pelos gráficos que o cruzamento das duas curvas se dará com $y_{\varepsilon \lambda}>\varepsilon$, o que implica que $\varphi_{\varepsilon}\left(\mathrm{y}_{\varepsilon \lambda}\right)=1$ e $\left[\varphi_{\varepsilon}\left(y_{\varepsilon \lambda}\right)\right]^{\prime}=0$.

Logo,

$$
\Delta_{\mu}\left(\mathrm{y}_{\varepsilon \lambda}\right)=(0 / 1)^{2}+0(-2 \sigma-4 \mathrm{k})+1^{2}\left(\sigma^{2}-4 \Delta\right)=\sigma^{2}-4 \Delta<0 .
$$

Portanto, a singularidade $\left(\mathrm{x}_{\varepsilon \lambda}, \mathrm{y}_{\varepsilon \lambda}\right)$ é um foco hiperbólico do campo regularizado $Z_{\varepsilon \lambda}$, onde $\lambda=\varepsilon \Delta+\mathrm{k}$.

Afirma-se que, diminuindo-se $\varepsilon>0$, se necessário, para $\lambda$ tal que $o$ ponto de cruzamento dos gráficos $y_{\varepsilon \lambda}$ é o mesmo ponto em que $\varphi_{\varepsilon}^{\prime}$ atinge o seu máximo, tem-se $\Delta_{\mu}\left(\mathrm{y}_{\varepsilon \lambda}\right)>0$.

De fato, analisando-se a expressão

$$
\Delta_{\mu}\left(\mathrm{y}_{\varepsilon \lambda}\right)=\left(\left[\varphi_{\varepsilon}\left(\mathrm{y}_{\varepsilon \lambda}\right)\right]^{\prime} / \varphi_{\varepsilon}\left(\mathrm{y}_{\varepsilon \lambda}\right)\right)^{2}+\left[\varphi_{\varepsilon}\left(\mathrm{y}_{\varepsilon \lambda}\right)\right]^{\prime}(-2 \sigma-4 \mathrm{k})+\left(\varphi_{\varepsilon}\left(\mathrm{y}_{\varepsilon \lambda}\right)\right)^{2}\left(\sigma^{2}-4 \Delta\right),
$$

observa-se que o termo $\left(\left[\varphi_{\varepsilon}\left(\mathrm{y}_{\varepsilon \lambda}\right)\right]^{\prime} / \varphi_{\varepsilon}\left(\mathrm{y}_{\varepsilon \lambda}\right)\right)^{2}$ é positivo e crescente, conforme $\varepsilon$ tende a zero, além disto, cresce mais rapidamente que $\left[\varphi_{\varepsilon}\left(\mathrm{y}_{\varepsilon} \lambda\right)\right]^{\prime}$. Logo, existe $\varepsilon_{0}>0$ tal que se $0<\varepsilon<\varepsilon_{0}$, tem-se $\Delta_{\mu}\left(\mathrm{y}_{\varepsilon \lambda}\right)>0$.

Afirma-se que o ponto $\left(x_{\varepsilon \lambda}, y_{\varepsilon \lambda}\right)$, onde $x_{\varepsilon \lambda}$ vem da equação (B), é um nó hiperbólico de $Z_{\varepsilon \lambda}$. $\Delta_{\mu}<\mathrm{b}_{\mu}^{2}$

De acordo com a demonstração do Proposição 1.4, resta mostrar que Tem-se 


$$
\Delta_{\mu}=b_{\mu}{ }^{2}-4 a_{\mu} c_{\mu}<b_{\mu}^{2} \Rightarrow a_{\mu} c_{\mu}>0
$$

Da equação característica

$$
\left.\mu^{2}+\mu\left(\left[\varphi_{\varepsilon}(\mathrm{y})\right]^{\prime} / \varphi_{\varepsilon}(\mathrm{y})\right)-\sigma \varphi_{\varepsilon}(\mathrm{y})\right)+\varphi_{\varepsilon}(\mathrm{y})\left(\left[\varphi_{\varepsilon}(\mathrm{y})\right]^{\prime}\left(\mathrm{k} / \varphi_{\varepsilon}(\mathrm{y})\right)+\Delta \varphi_{\varepsilon}(\mathrm{y})\right)=0,
$$

tem-se $a_{\mu} c_{\mu}=\varphi_{\varepsilon}\left(y_{\varepsilon} \lambda\right)\left(\left[\varphi_{\varepsilon}\left(y_{\varepsilon} \lambda\right)\right]^{\prime}\left(k / \varphi_{\varepsilon}\left(y_{\varepsilon} \lambda\right)\right)+\Delta \varphi_{\varepsilon}\left(y_{\varepsilon} \lambda\right)\right)>0$.

Portanto, está provado que $\left(\mathrm{x}_{\varepsilon \lambda,}, \mathrm{y}_{\varepsilon \lambda}\right)$ é um nó hiperbólico de $Z_{\varepsilon \lambda}$.

Observa-se que $\mathrm{a}_{\mu} \mathrm{c}_{\mu}>0$ para qualquer ponto $\mathrm{y}$, portanto, toda singularidade que tiver $\Delta_{\mu}>0$ corresponderá a um nó hiperbólico e toda singularidade que tiver $\Delta_{\mu}<0$ corresponderá a um foco hiperbólico.

Resta provar que, fixado $\varepsilon>0$, com $0<\varepsilon<\varepsilon_{0}$, existe um valor $\lambda_{0}$ tal que a singularidade de $Z_{\varepsilon \lambda_{0}}$ possui um auto-valor duplo. De fato, como $\Delta_{\mu}(\mathrm{y})$ é uma função contínua, existe um ponto em que $\Delta_{\mu}=0$, que corresponde ao auto-valor duplo.

As figuras abaixo ilustram o campo vetorial descontínuo $Z_{\lambda}$ para os casos $\lambda>0, \lambda<0, \sigma>0$ e $\sigma<0$.

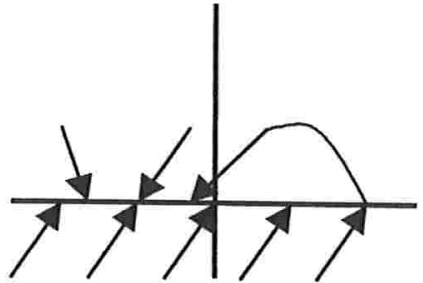

$\lambda<0$ e $\sigma>0$

Fig. 90

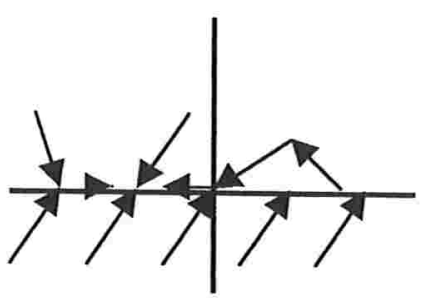

$\lambda<0$ e $\sigma<0$

Fig. 92

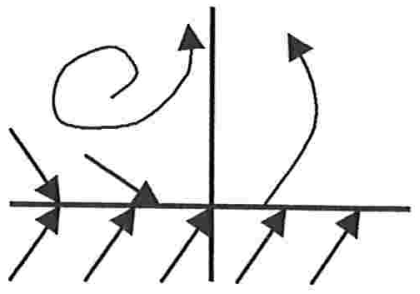

$\lambda>0$ e $\sigma>0$

Fig. 91

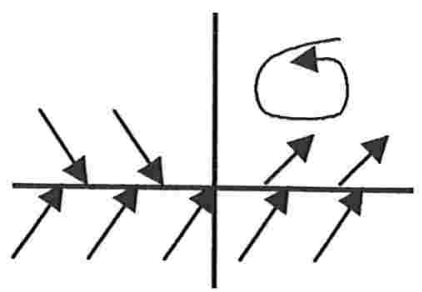

$\lambda>0$ e $\sigma<0$

Fig. 93

\subsection{Proposição}

Seja $Z_{\lambda}$ uma familia de campos vetoriais descontínuos com $k>0$, $\sigma^{2} \geq 4 \Delta>0$ (caso iii). Dada uma função de transição $\varphi$, para valores dos 
parâmetros $\varepsilon>0$ e $\lambda$ qualquer, o campo vetorial regularizado $Z_{\varepsilon, \lambda}$ possui um nó. Se $\sigma<0$, o nó é sempre hiperbólico e atrator, se $\sigma>0$, existe $\lambda_{0}=\lambda_{0}(\varepsilon)$ tal que o campo $Z_{\varepsilon, \lambda o}$ possui um nó não hiperbólico. Neste caso, se $\lambda>\lambda_{0}, Z_{\varepsilon, \lambda}$ possui um nó hiperbólico repulsor e se $\lambda<\lambda_{0}, Z_{\varepsilon, \lambda}$ possui um nó hiperbólico atrator.

demonstração

Tem-se

$$
\begin{aligned}
& \varphi_{\varepsilon}(\mathrm{y})=\mathrm{k} /(\mathrm{k}+\Delta \mathrm{y}-\lambda) \\
& \mathrm{e} \\
& \varphi_{\varepsilon}(\mathrm{y})=1 /(1-\mathrm{x}-\sigma \mathrm{y})
\end{aligned}
$$

Seja $g_{\lambda}(y)=k /(k+\Delta y-\lambda)$. Se existir y tal que $\varphi_{\varepsilon}(y)=g_{\lambda}(y)$, então este valor y será a ordenada de uma singularidade do campo $Z_{\varepsilon, \lambda}$.

Os gráficos de $g_{\lambda}(y)$ e de $\varphi_{\varepsilon}(y)$ são dados na figura abaixo:

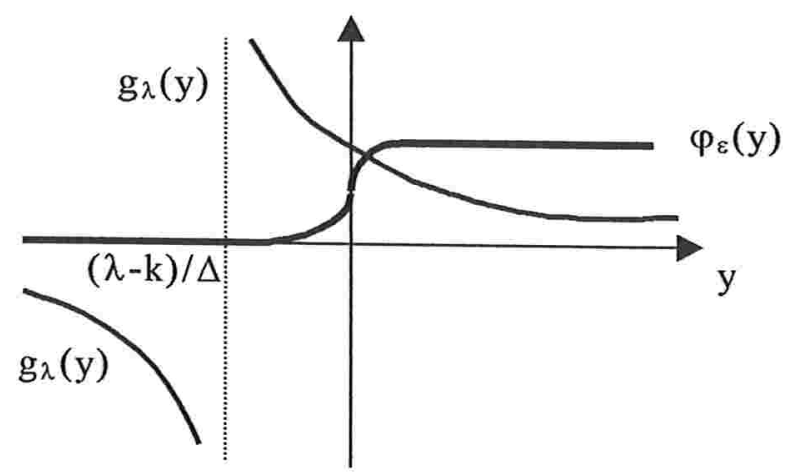

Fig. 94

Quando $\lambda$ cresce, a assíntota do gráfico de $\mathrm{g}_{\lambda}(\mathrm{y})$ se desloca para a direita, e quando $\lambda$ decresce, a assíntota do gráfico de $\mathrm{g}_{\lambda}(\mathrm{y})$ se desloca para a esquerda. Logo, para quaisquer $\varepsilon>0$ e $\lambda$, os gráficos de $g_{\lambda}(y)$ e $\varphi_{\varepsilon}(y)$ se cruzam num único ponto $\left(\mathrm{y}_{\varepsilon \lambda}, \mathrm{g}_{\lambda}\left(\mathrm{y}_{\varepsilon} \lambda\right)\right)=\left(\mathrm{y}_{\varepsilon \lambda}, \varphi_{\varepsilon}\left(\mathrm{y}_{\varepsilon \lambda}\right)\right)$.

Seja $\left(\mathrm{x}_{\varepsilon \lambda,}, \mathrm{y}_{\varepsilon}\right)$ o único ponto singular de $Z_{\varepsilon, \lambda}$.

Os auto-valores desta singularidade são

$\mu=1 / 2\left(-\left[\varphi_{\varepsilon}\left(\mathrm{y}_{0}\right)\right]^{\prime} / \varphi_{\varepsilon}\left(\mathrm{y}_{0}\right)+\sigma \varphi_{\varepsilon}\left(\mathrm{y}_{0}\right) \pm \sqrt{ } \Delta_{\mu}\right)$.

Onde $\Delta_{\mu}=\left(\left[\varphi_{\varepsilon}\left(\mathrm{y}_{0}\right)\right]^{\prime} / \varphi_{\varepsilon}\left(\mathrm{y}_{0}\right)\right)^{2}+\left[\varphi_{\varepsilon}\left(\mathrm{y}_{0}\right)\right]^{\prime}(-2 \sigma-4 \mathrm{k})+\left(\varphi_{\varepsilon}\left(\mathrm{y}_{0}\right)\right)^{2}\left(\sigma^{2}-4 \Delta\right)$.

Afirma-se que $\left(\mathrm{x}_{\varepsilon} \lambda, \mathrm{y}_{\varepsilon \lambda}\right)$ é um nó hiperbólico. 
Para provar esta afirmação, é necessário provar que $0 \leq \Delta_{\mu}<\mathrm{b}_{\lambda}{ }^{2}$.

Analogamente à demonstração da Proposição 1.4, tem-se

$$
\Delta_{\mu}<\mathrm{b}_{\mu}{ }^{2} \Rightarrow\left[\varphi_{\varepsilon}\left(\mathrm{y}_{\varepsilon \lambda}\right)\right]^{\prime}>-\Delta\left(\varphi_{\varepsilon}\left(\mathrm{y}_{\varepsilon \lambda}\right)\right)^{2} / \mathrm{k}=\left[\mathrm{g}_{\lambda}\left(\mathrm{y}_{\varepsilon \lambda}\right)\right]^{\prime}
$$

A desigualdade acima é sempre verdadeira, uma vez que $\left[\varphi_{\varepsilon}\left(\mathrm{y}_{\varepsilon \lambda}\right)\right]^{\prime} \geq 0$ e $\left[g_{\lambda}\left(y_{\varepsilon} \lambda\right)\right]^{\prime}<0$.

Resta verificar-se quando $\Delta_{\mu} \geq 0$.

De fato, para ter-se

$$
\Delta_{\mu}=\left(\left[\varphi_{\varepsilon}(\mathrm{y})\right]^{\prime} / \varphi_{\varepsilon}(\mathrm{y})\right)^{2}+\left[\varphi_{\varepsilon}(\mathrm{y})\right]^{\prime}(-2 \sigma-4 \mathrm{k})+\left(\varphi_{\varepsilon}(\mathrm{y})\right)^{2}\left(\sigma^{2}-4 \Delta\right)>0,
$$

depende do termo $\left[\varphi_{\varepsilon}(\mathrm{y})\right]^{\prime}(-2 \sigma-4 \mathrm{k})$.

Portanto, a singularidade $\left(\mathrm{x}_{\varepsilon \lambda,} \mathrm{y}_{\varepsilon \lambda}\right)$ é um nó hiperbólico de $Z_{\varepsilon, \lambda}$, para $\varepsilon>0$ e $\lambda$ qualquer quando

$$
\left(\left[\varphi_{\varepsilon}(\mathrm{y})\right]^{\prime} / \varphi_{\varepsilon}(\mathrm{y})\right)^{2}+\left(\varphi_{\varepsilon}(\mathrm{y})\right)^{2}\left(\sigma^{2}-4 \Delta\right)>\left[\varphi_{\varepsilon}(\mathrm{y})\right]^{\prime}(2 \sigma+4 \mathrm{k}) .
$$

Quando isto ocorre, a singularidade é atratora se

$$
\mathrm{b}_{\mu}=-\left[\varphi_{\varepsilon}(\mathrm{y})\right]^{\prime} / \varphi_{\varepsilon}(\mathrm{y})+\sigma \varphi_{\varepsilon}(\mathrm{y})<0,
$$

o que sempre é verdade se $\sigma<0$. E é repulsora se $b_{\mu}>0$.

As figuras abaixo ilustram o campo vetorial descontínuo $Z_{\lambda}$ para os $\operatorname{casos} \lambda>0, \lambda<0, \sigma>0$ e $\sigma<0$.

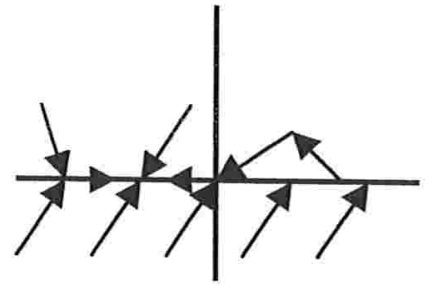

$\lambda<0$ e $\sigma>0$

Fig. 95

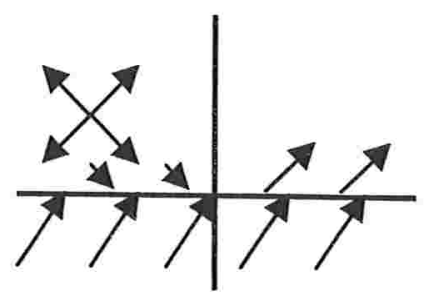

$\lambda>0$ e $\sigma>0$

Fig. 96 


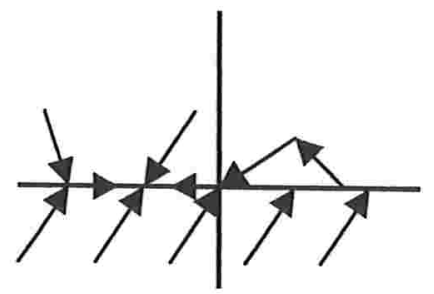

$\lambda<0$ e $\sigma<0$

Fig. 97

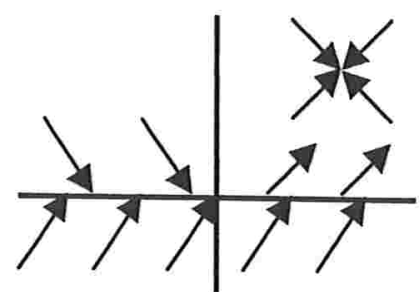

$\lambda>0$ e $\sigma<0$

Fig. 98

\subsection{Proposição}

Seja $Z_{\lambda}$ uma família de campos vetoriais descontinuos com $k<0$, $4 \Delta>\sigma^{2}>0$ (caso iv). Dada uma função de transição $\varphi$, a familia de campos vetoriais regularizados $Z_{\varepsilon, \lambda}$ possui valores dos parâmetros $\left(\varepsilon, \lambda_{0}(\varepsilon)\right)$, onde $\varepsilon>0$, para os quais $Z_{\varepsilon, \lambda o}$ possui uma singularidade não hiperbólica. Para $\lambda>\lambda_{0}$ os campos vetoriais regularizados $Z_{\varepsilon, \lambda}$ possuem duas singularidades, uma sela e um foco, ambos hiperbólicos, e para $\lambda<\lambda_{o}$, os campos vetoriais regularizados $Z_{\varepsilon, \lambda}$ não possuem singularidades.

demonstração

$$
\begin{aligned}
& \text { Tem-se } \\
& \varphi_{\varepsilon}(y)=k /(k+\Delta y-\lambda) \\
& \text { e } \\
& \varphi_{\varepsilon}(y)=1 /(1-x-\sigma y)
\end{aligned}
$$

Seja $g_{\lambda}(y)=k /(k+\Delta y-\lambda)$. Se existir y tal que $\varphi_{\varepsilon}(y)=g_{\lambda}(y)$, então este valor y será a ordenada de uma singularidade do campo $Z_{\varepsilon, \lambda}$. Os gráficos de $g_{0}(y)=k /(k+\Delta y)$ e de $\varphi_{\varepsilon}(y)$ são dados na figura abaixo:

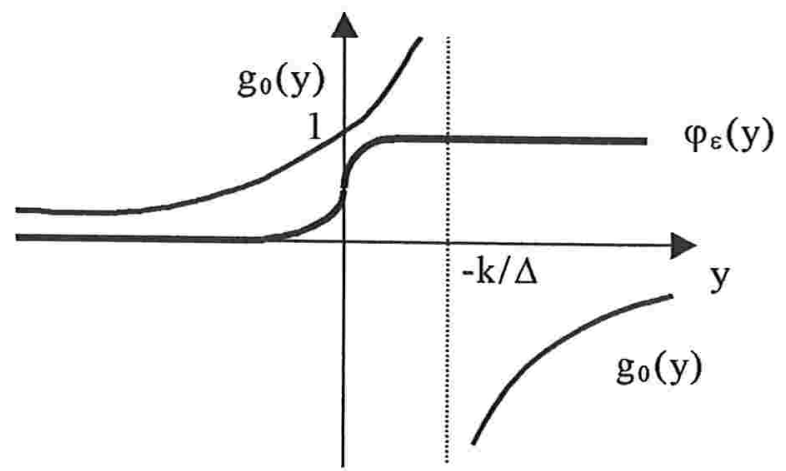

Fig. 99 
Quando $\lambda$ decresce, a assíntota do gráfico de $\mathrm{g}_{\lambda}(\mathrm{y})$ se desloca para a esquerda, logo o gráfico de $g_{\lambda}(y)$ não cruzará o gráfico de $\varphi_{\varepsilon}(\mathrm{y})$. Quando $\lambda$ cresce, a assíntota do gráfico de $\mathrm{g}_{\lambda}(\mathrm{y})$ se desloca para a direita. Logo, mantendo-se $\varepsilon$ fixo, existe um único valor $\lambda_{0}=\lambda_{0}(\varepsilon)>0$ para o qual os gráficos de $\mathrm{g}_{\lambda}(\mathrm{y})$ e $\varphi_{\varepsilon}(\mathrm{y})$ se tangenciam num único ponto $\left(\mathrm{y}_{0}, \mathrm{~g}_{\lambda_{0}}\left(\mathrm{y}_{0}\right)\right)=$ $\left(\mathrm{y}_{0}, \varphi_{\varepsilon}\left(\mathrm{y}_{0}\right)\right)$, como ilustra a figura abaixo:

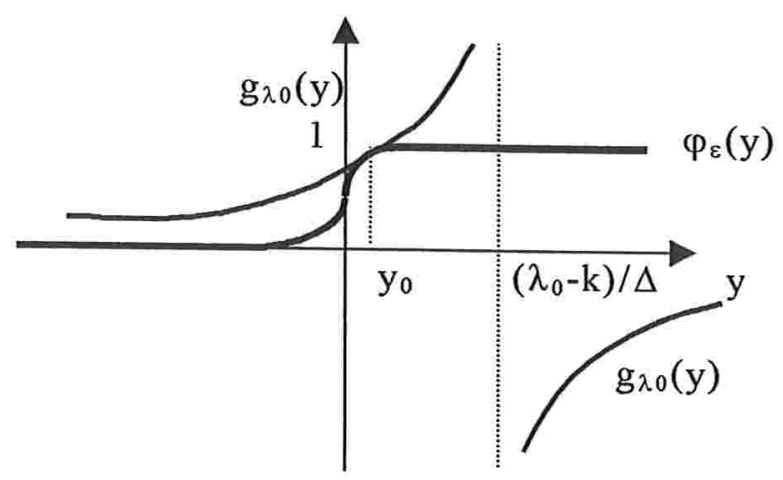

Fig. 100

Para $\lambda>\lambda_{0}$ os gráficos de $g_{\lambda}(y)$ e $\varphi_{\varepsilon}(y)$ se cruzarão duas vezes, nos pontos $\left(\mathrm{y}_{1}, \mathrm{~g}_{\lambda}\left(\mathrm{y}_{1}\right)\right)=\left(\mathrm{y}_{1}, \varphi_{\varepsilon}\left(\mathrm{y}_{1}\right)\right)$ e $\left(\mathrm{y}_{2}, \mathrm{~g}_{\lambda}\left(\mathrm{y}_{2}\right)\right)=\left(\mathrm{y}_{2}, \varphi_{\mathrm{s}}\left(\mathrm{y}_{2}\right)\right)$, como ilustra a figura abaixo:

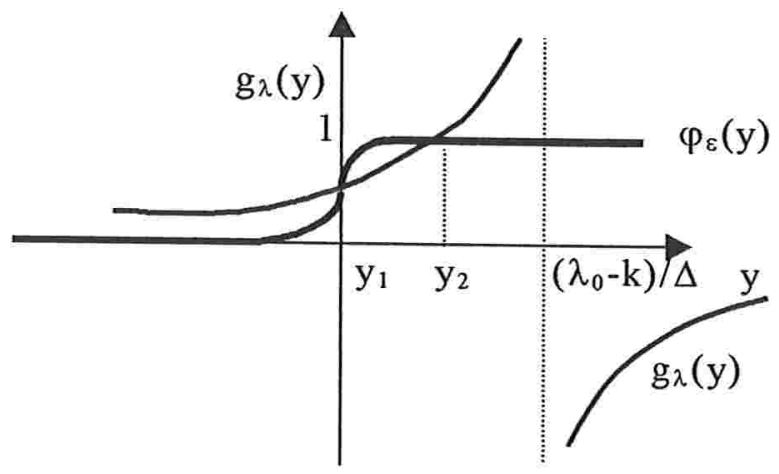

Fig. 101

Seja $\left(\mathrm{x}_{0}, \mathrm{y}_{0}\right)$ a solução das equações (A) e (B), onde $\left(\mathrm{y}_{0}, \mathrm{~g}_{\lambda_{0}}\left(\mathrm{y}_{0}\right)\right)=$ $\left(\mathrm{y}_{0}, \varphi_{\varepsilon}\left(\mathrm{y}_{0}\right)\right)$ é o ponto de tangência entre os gráficos de $g_{\lambda_{0}}$ e $\varphi_{\varepsilon}$ ilustrado na figura 100, ou seja, $(x, y)$ é a única singularidade de $Z_{\varepsilon, \lambda 0}$.

Afirma-se que $\left(\mathrm{x}_{0}, \mathrm{y}_{0}\right)$ é uma singularidade não hiperbólica de $Z_{8,20}$.

De fato, como os gráficos de $\mathrm{g}_{\lambda}(\mathrm{y})$ e $\varphi_{\mathrm{\varepsilon}}(\mathrm{y})$ se tangenciam para $\mathrm{y}=\mathrm{y}_{0}$, então $\left(\mathrm{g}_{20}\left(\mathrm{y}_{0}\right)\right)^{\prime}=\left(\varphi_{\varepsilon}\left(\mathrm{y}_{0}\right)\right)^{\prime}$, isto é, 


$$
\left(\varphi_{\varepsilon}\left(\mathrm{y}_{0}\right)\right)^{\prime}=-\Delta \mathrm{k} /\left(\mathrm{k}+\Delta \mathrm{y}_{0}-\lambda\right)^{2}=-\Delta\left(\varphi_{\varepsilon}\left(\mathrm{y}_{0}\right)\right)^{2} / \mathrm{k}
$$

Logo os auto-valores desta singularidade são

$$
\mu=1 / 2\left(-\left[\varphi_{\varepsilon}\left(\mathrm{y}_{0}\right)\right]^{\prime} / \varphi_{\varepsilon}\left(\mathrm{y}_{0}\right)+\sigma \varphi_{\varepsilon}\left(\mathrm{y}_{0}\right) \pm \sqrt{ } \Delta_{\mu}\right)
$$

Onde $\Delta_{\mu}=\left(\left[\varphi_{\varepsilon}\left(\mathrm{y}_{0}\right)\right]^{\prime} / \varphi_{\varepsilon}\left(\mathrm{y}_{0}\right)\right)^{2}+\left[\varphi_{\varepsilon}\left(\mathrm{y}_{0}\right)\right]^{\prime}(-2 \sigma-4 \mathrm{k})+\left(\varphi_{\varepsilon}\left(\mathrm{y}_{0}\right)\right)^{2}\left(\sigma^{2}-4 \Delta\right)$.

Usando-se (C) tem-se

$$
\begin{aligned}
& \Delta_{\mu}=\left(-\Delta\left(\varphi_{\varepsilon}\left(\mathrm{y}_{0}\right)\right)^{2} / \mathrm{k} \varphi_{\varepsilon}\left(\mathrm{y}_{0}\right)\right)^{2}-\Delta\left(\varphi_{\varepsilon}\left(\mathrm{y}_{0}\right)\right)^{2}(-2 \sigma-4 \mathrm{k}) / \mathrm{k}+\left(\varphi_{\varepsilon}\left(\mathrm{y}_{0}\right)\right)^{2}\left(\sigma^{2}-4 \Delta\right) \\
& =\Delta^{2}\left(\varphi_{\varepsilon}\left(\mathrm{y}_{0}\right)\right)^{2} / \mathrm{k}^{2}+\left(\varphi_{\varepsilon}\left(\mathrm{y}_{0}\right)\right)^{2}\left(2 \Delta \sigma / \mathrm{k}+4 \Delta+\sigma^{2}-4 \Delta\right)= \\
& =\Delta^{2}\left(\varphi_{\varepsilon}\left(\mathrm{y}_{0}\right)\right)^{2} / \mathrm{k}^{2}+\left(\varphi_{\varepsilon}\left(\mathrm{y}_{0}\right)\right)^{2}\left(2 \Delta \sigma / \mathrm{k}+\sigma^{2}\right)= \\
& =\left(\varphi_{\varepsilon}\left(\mathrm{y}_{0}\right)\right)^{2}\left(\Delta^{2} / \mathrm{k}^{2}+2 \Delta \sigma / \mathrm{k}+\sigma^{2}\right)=\left(\varphi_{\varepsilon}\left(\mathrm{y}_{0}\right)\right)^{2}(\Delta / \mathrm{k}+\sigma)^{2} \\
& \mu=1 / 2\left(\Delta\left(\varphi_{\varepsilon}\left(\mathrm{y}_{0}\right)\right)^{2} / \mathrm{k} \varphi_{\varepsilon}\left(\mathrm{y}_{0}\right)+\sigma \varphi_{\varepsilon}\left(\mathrm{y}_{0}\right) \pm \sqrt{ } \Delta_{\mu}\right) \\
& =1 / 2\left(\Delta \varphi_{\varepsilon}\left(\mathrm{y}_{0}\right) / \mathrm{k}+\sigma \varphi_{\varepsilon}\left(\mathrm{y}_{0}\right) \pm \sqrt{ }\left(\left(\varphi_{\varepsilon}\left(\mathrm{y}_{0}\right)\right)^{2}(\Delta / \mathrm{k}+\sigma)^{2}\right)\right)= \\
& =1 / 2\left(\varphi_{\varepsilon}\left(\mathrm{y}_{0}\right)(\Delta / \mathrm{k}+\sigma) \pm \varphi_{\varepsilon}\left(\mathrm{y}_{0}\right) \sqrt{ }(\Delta / \mathrm{k}+\sigma)^{2}\right)= \\
& =1 / 2 \varphi_{\varepsilon}\left(\mathrm{y}_{0}\right)((\Delta / \mathrm{k}+\sigma) \pm|\Delta / \mathrm{k}+\sigma|) \\
& \Rightarrow \text { os auto-valores são } \mu_{0}=0 \text { e } \mu_{1}=\varphi_{\varepsilon}\left(\mathrm{y}_{0}\right)(\Delta / \mathrm{k}+\sigma)
\end{aligned}
$$
de $Z_{\varepsilon, \lambda 0}$.

Portanto, a singularidade $\left(\mathrm{x}_{0}, \mathrm{y}_{0}\right)$ é uma singularidade não hiperbólica

Afirma-se que se $\lambda>\lambda_{0}, Z_{\varepsilon, \lambda}$ tem dois pontos singulares, um sendo sela hiperbólica e o outro um foco hiperbólico.

De fato, se $\lambda>\lambda_{0}$, como pode ser observado na figura 101 , os gráficos de $\mathrm{g}_{\lambda}(\mathrm{y})$ e $\varphi_{\varepsilon}(\mathrm{y})$ se cruzam duas vezes, nos pontos $\left(\mathrm{y}_{1}, \mathrm{~g}_{\lambda}\left(\mathrm{y}_{1}\right)\right)=\left(\mathrm{y}_{1}\right.$, $\left.\varphi_{\varepsilon}\left(\mathrm{y}_{1}\right)\right)$ e $\left(\mathrm{y}_{2}, \mathrm{~g}_{\lambda}\left(\mathrm{y}_{2}\right)\right)=\left(\mathrm{y}_{2}, \varphi_{\varepsilon}\left(\mathrm{y}_{2}\right)\right)$. Através da equação (B) encontra-se os valores $\mathrm{x}_{1}$ e $\mathrm{x}_{2}$ correspondentes a $\mathrm{y}_{1}$ e $\mathrm{y}_{2}$, isto é, tais que os pares $\left(\mathrm{x}_{1}, \mathrm{y}_{1}\right) \mathrm{e}$ $\left(\mathrm{x}_{2}, \mathrm{y}_{2}\right)$ são as únicas soluções de (A) e (B), e portanto são singularidades de $Z_{\varepsilon, \lambda}$. Resta agora investigar a natureza destas singularidades.

Observa-se que

$$
\left(\varphi_{\varepsilon}\left(\mathrm{y}_{1}\right)\right)^{\prime}>\left(\mathrm{g}_{\lambda}\left(\mathrm{y}_{1}\right)\right)^{\prime}=-\Delta \mathrm{k} /\left(\mathrm{k}+\Delta \mathrm{y}_{1}-\lambda\right)^{2}=-\Delta\left(\varphi_{\varepsilon}\left(\mathrm{y}_{1}\right)\right)^{2} / \mathrm{k}
$$


e

$\left(\varphi_{\varepsilon}\left(\mathrm{y}_{2}\right)\right)^{\prime}<\left(\mathrm{g}_{\lambda}\left(\mathrm{y}_{2}\right)\right)^{\prime}=-\Delta \mathrm{k} /\left(\mathrm{k}+\Delta \mathrm{y}_{2}-\lambda\right)^{2}=-\Delta\left(\varphi_{\varepsilon}\left(\mathrm{y}_{2}\right)\right)^{2} / \mathrm{k}$.

Observa-se que para que uma singularidade com auto-valores dados por

$\mu=1 / 2\left(-b_{\mu} \pm \sqrt{ } \Delta_{\mu}\right)$

ser uma sela hiperbólica deve-se ter

$\left.\begin{array}{l}-b_{\mu}+\sqrt{ } \Delta_{\mu}>0 \Rightarrow \sqrt{ } \Delta_{\mu}>b_{\mu} \\ -b_{\mu}-\sqrt{ } \Delta_{\mu}<0 \Rightarrow \sqrt{ } \Delta_{\mu}>-b_{\mu}\end{array}\right\} \quad \Rightarrow \sqrt{ } \Delta_{\mu}>\left|b_{\mu}\right| \Rightarrow \Delta_{\mu}>b_{\mu}{ }^{2}$.

E para esta singularidade ser um foco hiperbólico deve-se ter

$-\mathrm{b}_{\mu} \neq 0$ e $\Delta_{\mu}<0$.

Impondo-se $\Delta_{\mu}>\mathrm{b}_{\lambda}^{2}$ tem-se

$\Delta_{\mu}=\left(\left[\varphi_{\varepsilon}(\mathrm{y})\right]^{\prime} / \varphi_{\varepsilon}(\mathrm{y})\right)^{2}+\left[\varphi_{\varepsilon}(\mathrm{y})\right]^{\prime}(-2 \sigma-4 \mathrm{k})+\left(\varphi_{\varepsilon}(\mathrm{y})\right)^{2}\left(\sigma^{2}-4 \Delta\right)>$

$>\left(-\left[\varphi_{\mathrm{s}}(\mathrm{y})\right]^{\prime} / \varphi_{\mathrm{s}}(\mathrm{y})+\sigma \varphi_{\mathrm{\varepsilon}}(\mathrm{y})\right)^{2}=\mathrm{b}_{\lambda}{ }^{2}$

$\Rightarrow\left(\left[\varphi_{\varepsilon}(\mathrm{y})\right]^{\prime} / \varphi_{\varepsilon}(\mathrm{y})\right)^{2}+\left[\varphi_{\varepsilon}(\mathrm{y})\right]^{\prime}(-2 \sigma-4 \mathrm{k})+\left(\varphi_{\varepsilon}(\mathrm{y})\right)^{2}\left(\sigma^{2}-4 \Delta\right)>$

$>\left(\left[\varphi_{\varepsilon}(\mathrm{y})\right]^{\prime} / \varphi_{\varepsilon}(\mathrm{y})\right)^{2}-2 \sigma\left[\varphi_{\varepsilon}(\mathrm{y})\right]^{\prime}+\sigma^{2}\left(\varphi_{\varepsilon}\left(\varphi_{\varepsilon}(\mathrm{y})\right)^{2}\right.$

$\Rightarrow\left[\varphi_{\varepsilon}(\mathrm{y})\right]^{\prime}(-4 \mathrm{k})+\left(\varphi_{\varepsilon}(\mathrm{y})\right)^{2}(-4 \Delta)>0$

$\Rightarrow\left[\varphi_{\varepsilon}(\mathrm{y})\right]^{\prime} \mathrm{k}+\left(\varphi_{\varepsilon}(\mathrm{y})\right)^{2} \Delta<0$

$\Rightarrow\left[\varphi_{\varepsilon}(\mathrm{y})\right]^{\prime}>-\Delta\left(\varphi_{\varepsilon}(\mathrm{y})\right)^{2} / \mathrm{k}$.

Por (D), a desigualdade acima ocorre em $y=y_{1}$ pode-se concluir que a singularidade $\left(\mathrm{x}_{1}, \mathrm{y}_{1}\right)$ é uma sela hiperbólica de $Z_{\varepsilon, \lambda}$.

Afirma-se que $\Delta_{\mu}<0$ e $\mathrm{b}_{\mu} \neq 0$ em $\mathrm{y}_{2}$.

De fato,

$\Delta_{\mu}<0$ e $b_{\mu} \neq 0 \Rightarrow \Delta_{\mu}<0<b_{\mu}{ }^{2} \Rightarrow \Delta_{\mu}<b_{\mu}{ }^{2}$

Impondo-se $\Delta_{\mu}>\mathrm{b}_{\mu}{ }^{2}$ tem-se

$\left[\varphi_{\varepsilon}(\mathrm{y})\right]^{\prime}<-\Delta\left(\varphi_{\varepsilon}(\mathrm{y})\right)^{2} / \mathrm{k}$ 
Por (E), a desigualdade acima ocorre em $y=y_{2}$ pode-se concluir que a singularidade $\left(\mathrm{x}_{2}, \mathrm{y}_{2}\right)$ é um foco hiperbólico de $\mathrm{Z}_{\varepsilon, \lambda}$.

Afirma-se que se $\lambda<\lambda_{0}, Z_{\varepsilon, \lambda}$ não possui pontos singulares.

De fato, a afirmação acima decorre de não haver um cruzamento entre os gráficos de $g_{\lambda}(y)$ e $\varphi_{\varepsilon}(y)$ e consequentemente as equações (A) e (B) não possuírem soluções.

As figuras abaixo ilustram o campo vetorial descontínuo $Z_{\lambda}$ para os $\operatorname{casos} \lambda>0, \lambda<0, \sigma>0$ e $\sigma<0$.

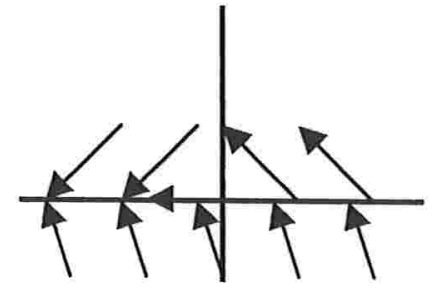

$\lambda<0$ e $\sigma>0$

Fig. 102

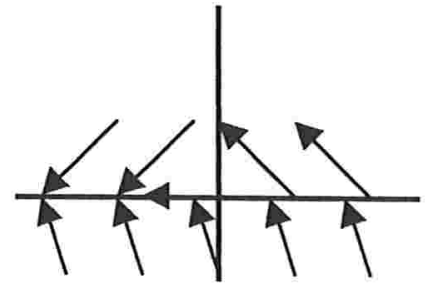

$\lambda<0$ e $\sigma<0$

Fig. 104

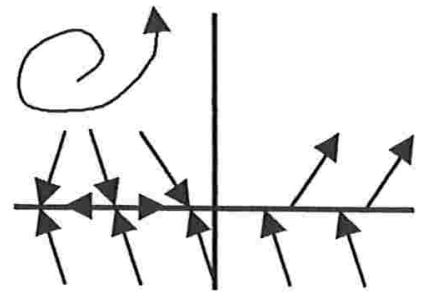

$\lambda>0$ e $\sigma>0$

Fig. 103

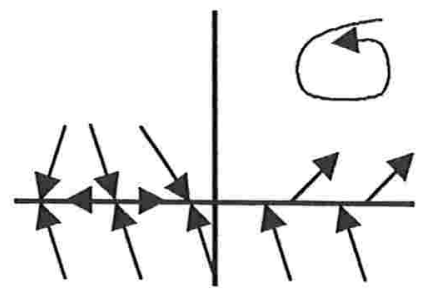

$\lambda>0$ e $\sigma<0$

Fig. 105

\subsection{Proposição}

Seja $Z_{\lambda}$ uma família de campos vetoriais descontínuos com $k<0 e$ $\sigma^{2} \geq 4 \Delta>0$ (caso v). Dada uma função de transição $\varphi$, a família de campos vetoriais regularizados $Z_{\varepsilon, \lambda}$ possui valores dos parâmetros $\left(\varepsilon, \lambda_{0}(\varepsilon)\right)$, onde $\varepsilon>0$, para os quais $Z_{\varepsilon, \lambda o}$ possui uma singularidade tipo sela-nó. Para $\lambda>\lambda_{0}$ os campos vetoriais regularizados $Z_{\varepsilon, \lambda}$ possuem duas singularidades, uma sela e um nó, ambos hiperbólicos, e para $\lambda<\lambda_{0}$, os campos vetoriais regularizados $Z_{\varepsilon, \lambda}$ não possuem singularidades.

demonstração

Tem-se 


$$
\begin{aligned}
& \varphi_{\varepsilon}(\mathrm{y})=\mathrm{k} /(\mathrm{k}+\Delta \mathrm{y}-\lambda) \\
& \mathrm{e} \\
& \varphi_{\varepsilon}(\mathrm{y})=1 /(1-\mathrm{x}-\sigma \mathrm{y})
\end{aligned}
$$

Seja $g_{\lambda}(y)=k /(k+\Delta y-\lambda)$. Se existir y tal que $\varphi_{\varepsilon}(y)=g_{\lambda}(y)$, então este valor y será a ordenada de uma singularidade do campo $Z_{\varepsilon, \lambda}$. abaixo:

Os gráficos de $\mathrm{g}_{0}(\mathrm{y})=\mathrm{k} /(\mathrm{k}+\Delta \mathrm{y})$ e de $\varphi_{\varepsilon}(\mathrm{y})$ são dados na figura

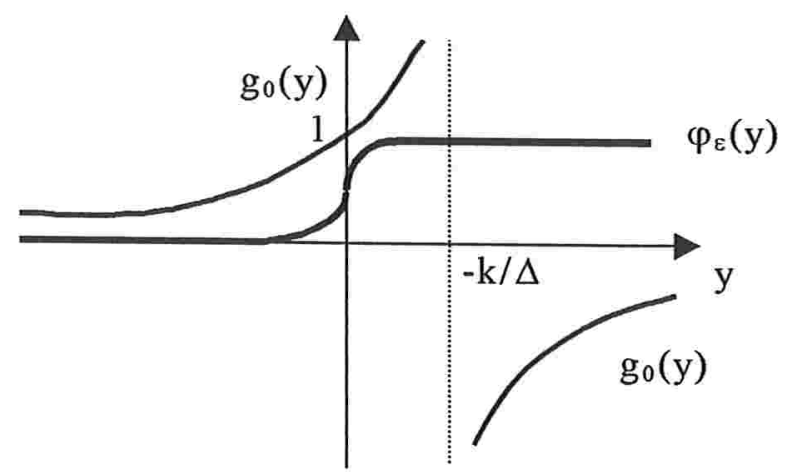

Fig. 106

Quando $\lambda$ decresce, a assíntota do gráfico de $\mathrm{g}_{\lambda}(\mathrm{y})$ se desloca para a esquerda, $\log 0$ o gráfico de $\mathrm{g}_{\lambda}(\mathrm{y})$ não cruzará o gráfico de $\varphi_{\varepsilon}(\mathrm{y})$. Quando $\lambda$ cresce, a assíntota do gráfico de $\mathrm{g}_{\lambda}(\mathrm{y})$ se desloca para a direita, logo, mantendo-se $\varepsilon$ fixo, existe um único valor $\lambda_{0}=\lambda_{0}(\varepsilon)>0$ para o qual os gráficos de $\mathrm{g}_{\lambda}(\mathrm{y})$ e $\varphi_{\mathrm{s}}(\mathrm{y})$ se tangenciam num único ponto $\left(\mathrm{y}_{0}, \mathrm{~g}_{\lambda_{0}}\left(\mathrm{y}_{0}\right)\right)=$ $\left(\mathrm{y}_{0}, \varphi_{\varepsilon}\left(\mathrm{y}_{0}\right)\right)$, como ilustra a figura abaixo:

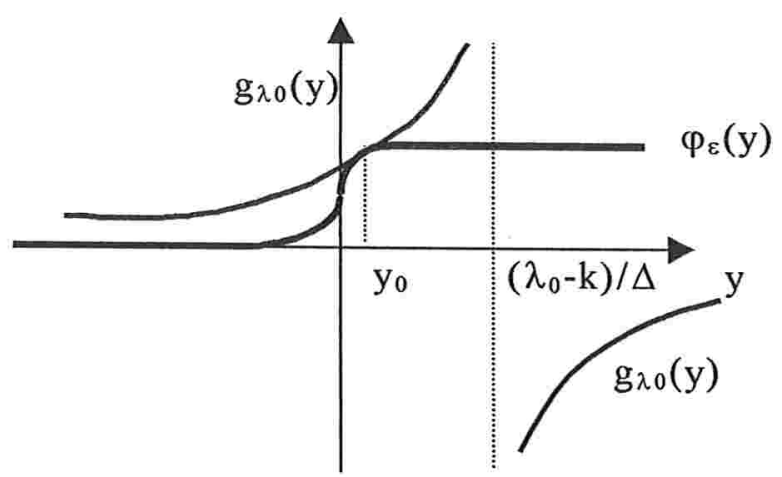

Fig. 107

Para $\lambda>\lambda_{0}$ os gráficos de $\mathrm{g}_{\lambda}(\mathrm{y})$ e $\varphi_{\varepsilon}(\mathrm{y})$ se cruzarão duas vezes, nos pontos $\left(\mathrm{y}_{1}, \mathrm{~g}_{\lambda}\left(\mathrm{y}_{1}\right)\right)=\left(\mathrm{y}_{1}, \varphi_{\varepsilon}\left(\mathrm{y}_{1}\right)\right)$ e $\left(\mathrm{y}_{2}, \mathrm{~g}_{\lambda}\left(\mathrm{y}_{2}\right)\right)=\left(\mathrm{y}_{2}, \varphi_{\varepsilon}\left(\mathrm{y}_{2}\right)\right)$, como ilustra a figura abaixo: 


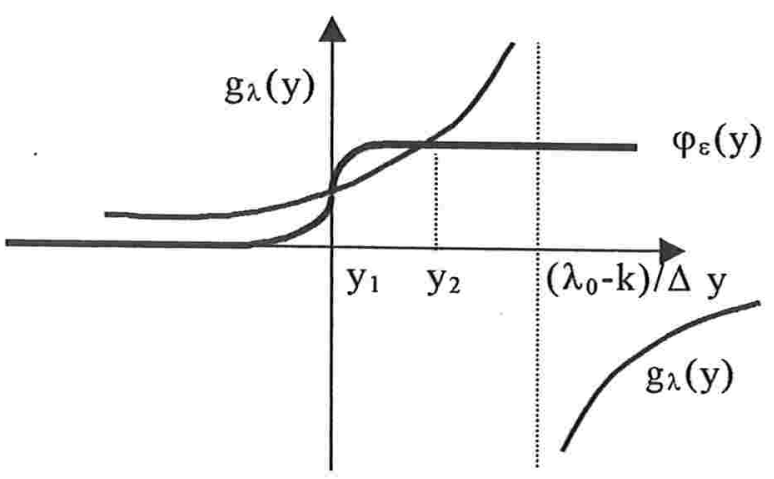

Fig. 108

Seja $\left(\mathrm{x}_{0}, \mathrm{y}_{0}\right)$ a solução das equações (A) e (B), onde $\left(\mathrm{y}_{0}, \mathrm{~g}_{\lambda_{0}}\left(\mathrm{y}_{0}\right)\right)=$ $\left(\mathrm{y}_{0}, \varphi_{\varepsilon}\left(\mathrm{y}_{0}\right)\right)$ é o ponto de tangência entre os gráficos de $g_{\lambda 0}$ e $\varphi_{\mathrm{s}}$ ilustrado na figura 107, ou seja, $\left(\mathrm{x}_{0}, \mathrm{y}_{0}\right)$ é a única singularidade de $Z_{\varepsilon, 20}$.

Sejam $\left(\mathrm{x}_{1}, \mathrm{y}_{1}\right)$ e $\left(\mathrm{x}_{2}, \mathrm{y}_{2}\right)$ as soluções de (A) e (B) para $\lambda>\lambda_{0}$, onde $\mathrm{y}_{1}$ e $\mathrm{y}_{2}$ são as abscissas dos pontos de cruzamento dos gráficos de $g_{\lambda}(\mathrm{y})$ e $\varphi_{\varepsilon}(\mathrm{y})$, observados na figura 108.

O restante da demonstração é análogo ao que foi feito na demonstração da Proposição 1.4 (caso i). A conclusão, neste caso, é que $\left(\mathrm{x}_{0}, \mathrm{y}_{0}\right)$ é uma sela-nó de $Z_{\varepsilon \lambda 0}$; para $\lambda>\lambda_{0}$, os campos vetoriais regularizados $Z_{\varepsilon \lambda}$ possuem duas singularidades: $\left(\mathrm{x}_{1}, \mathrm{y}_{1}\right)$ uma sela e $\left(\mathrm{x}_{2}, \mathrm{y}_{2}\right)$ um nó, ambos hiperbólicos; e para $\lambda<\lambda_{0}$, os campos vetoriais regularizados $Z_{\varepsilon \lambda}$ não possuem singularidades.

As figuras abaixo ilustram o campo vetorial descontínuo $Z_{\lambda}$ para os $\operatorname{casos} \lambda>0, \lambda<0, \sigma>0$ e $\sigma<0$.

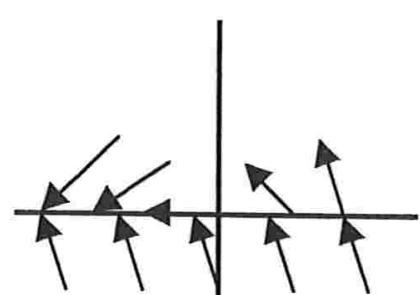

$\lambda<0$ e $\sigma>0$

Fig. 109

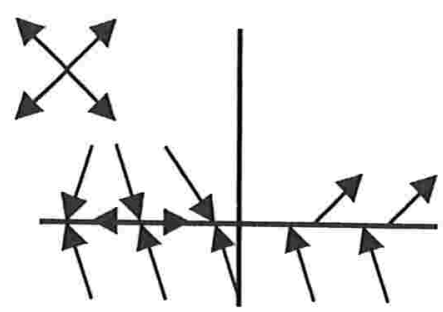

$\lambda>0$ e $\sigma>0$

Fig. 110 


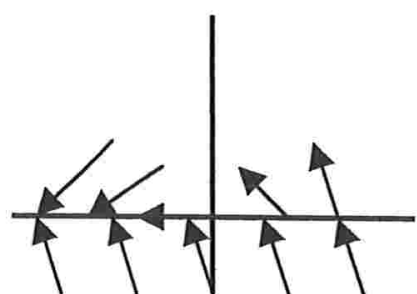

$\lambda<0$ e $\sigma<0$

Fig. 111

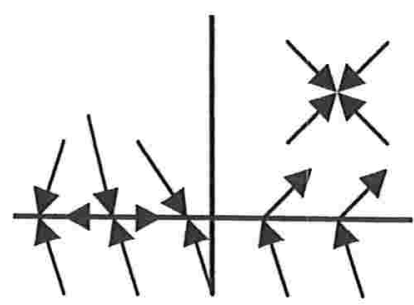

$\lambda>0$ e $\sigma<0$

Fig. 112

\subsection{Proposição}

Seja $Z_{\lambda}$ uma família de campos vetoriais descontínuos com $k<0$ e $\Delta<0$ (caso vi). Dada uma função de transição $\varphi$, para $\varepsilon>0$ e $\lambda$ qualquer, $o$ campo vetorial regularizado $Z_{\varepsilon, \lambda}$ possui uma sela hiperbólica.

demonstração

$$
\begin{aligned}
& \text { Tem-se } \\
& \varphi_{\varepsilon}(\mathrm{y})=\mathrm{k} /(\mathrm{k}+\Delta \mathrm{y}-\lambda) \\
& \mathrm{e} \\
& \varphi_{\varepsilon}(\mathrm{y})=1 /(1-\mathrm{x}-\sigma \mathrm{y})
\end{aligned}
$$

Seja $g_{\lambda}(y)=k /(k+\Delta y-\lambda)$. Se existir y tal que $\varphi_{s}(y)=g_{\lambda}(y)$, então este valor y será a ordenada de uma singularidade do campo $Z_{\varepsilon, \lambda}$.

Os gráficos de $g_{\lambda}(y)$ e de $\varphi_{\varepsilon}(y)$ são dados na figura abaixo:

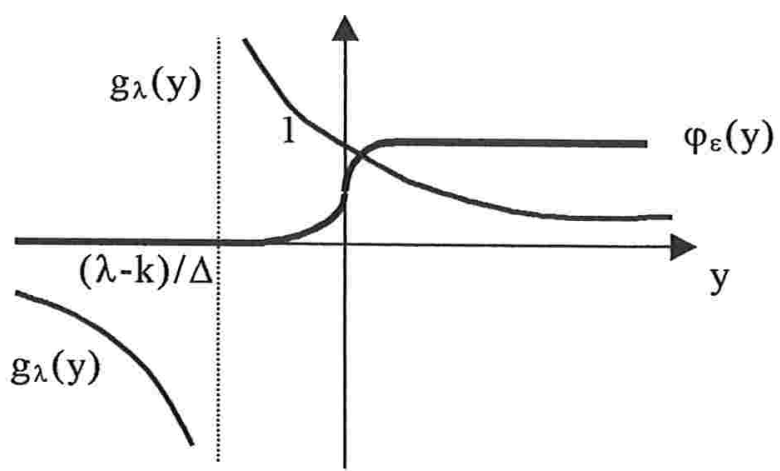

Fig. 113

Quando $\lambda$ cresce, a assíntota do gráfico de $\mathrm{g}_{\lambda}(\mathrm{y})$ se desloca para a esquerda e quando $\lambda$ decresce, para a direita, logo, mantendo-se $\varepsilon$ fixo, 
existe um único valor $\lambda_{0}=\lambda_{0}(\varepsilon)<0$ para o qual os gráficos de $g_{\lambda}(y)$ e $\varphi_{\varepsilon}(y)$ se cruzam num único ponto $\left(\mathrm{y}_{\varepsilon}, \mathrm{g}_{\lambda}\left(\mathrm{y}_{\varepsilon}\right)\right)=\left(\mathrm{y}_{\varepsilon \lambda}, \varphi_{\varepsilon}\left(\mathrm{y}_{\varepsilon} \lambda\right)\right)$.

Seja $\left(\mathrm{x}_{\varepsilon \lambda}, \mathrm{y}_{\varepsilon \lambda}\right)$ o ponto singular de $\mathrm{Z}_{\varepsilon, \lambda 0}$.

Os auto-valores desta singularidade são

$\mu=1 / 2\left(-\left[\varphi_{\varepsilon}\left(\mathrm{y}_{0}\right)\right]^{\prime} / \varphi_{\varepsilon}\left(\mathrm{y}_{0}\right)+\sigma \varphi_{\varepsilon}\left(\mathrm{y}_{0}\right) \pm \sqrt{ } \Delta_{\mu}\right)$

Onde $\Delta_{\mu}=\left(\left[\varphi_{\varepsilon}\left(\mathrm{y}_{0}\right)\right]^{\prime} / \varphi_{\varepsilon}\left(\mathrm{y}_{0}\right)\right)^{2}+\left[\varphi_{\varepsilon}\left(\mathrm{y}_{0}\right)\right]^{\prime}(-2 \sigma-4 \mathrm{k})+\left(\varphi_{\varepsilon}\left(\mathrm{y}_{0}\right)\right)^{2}\left(\sigma^{2}-4 \Delta\right)$.

Afirma-se que $\left(\mathrm{x}_{\varepsilon} \lambda, \mathrm{y}_{\varepsilon \lambda}\right)$ é uma sela hiperbólica de $Z_{\varepsilon, \lambda}$.

Para se provar esta afirmação, é necessário que se prove que $\Delta_{\mu}>\mathrm{b}_{\mu}{ }^{2}$.

Impondo-se $\Delta_{\mu}>\mathrm{b}_{\mu}{ }^{2}$ tem-se

$$
\begin{aligned}
& \begin{array}{l}
\Delta_{\mu}=\left(\left[\varphi_{\varepsilon}(\mathrm{y})\right]^{\prime} / \varphi_{\varepsilon}(\mathrm{y})\right)^{2}+\left[\varphi_{\varepsilon}(\mathrm{y})\right]^{\prime}(-2 \sigma-4 \mathrm{k})+\left(\varphi_{\varepsilon}(\mathrm{y})\right)^{2}\left(\sigma^{2}-4 \Delta\right)> \\
\quad>\left(-\left[\varphi_{\varepsilon}(\mathrm{y})\right]^{\prime} / \varphi_{\varepsilon}(\mathrm{y})+\sigma \varphi_{\varepsilon}(\mathrm{y})\right)^{2}=\mathrm{b}_{\lambda}{ }^{2}
\end{array} \\
& \Rightarrow\left(\left[\varphi_{\varepsilon}(\mathrm{y})\right]^{\prime} / \varphi_{\varepsilon}(\mathrm{y})\right)^{2}+\left[\varphi_{\varepsilon}(\mathrm{y})\right]^{\prime}(-2 \sigma-4 \mathrm{k})+\left(\varphi_{\varepsilon}(\mathrm{y})\right)^{2}\left(\sigma^{2}-4 \Delta\right)> \\
& \quad>\left(\left[\varphi_{\varepsilon}(\mathrm{y})\right]^{\prime} / \varphi_{\varepsilon}(\mathrm{y})\right)^{2}-2 \sigma\left[\varphi_{\varepsilon}(\mathrm{y})\right]^{\prime}+\sigma^{2}\left(\varphi_{\varepsilon}\left(\varphi_{\varepsilon}(\mathrm{y})\right)^{2}\right. \\
& \Rightarrow\left[\varphi_{\varepsilon}(\mathrm{y})\right]^{\prime}(-4 \mathrm{k})+\left(\varphi_{\varepsilon}(\mathrm{y})\right)^{2}(-4 \Delta)>0 \\
& \Rightarrow\left[\varphi_{\varepsilon}(\mathrm{y})\right]^{\prime} \mathrm{k}+\left(\varphi_{\varepsilon}(\mathrm{y})\right)^{2} \Delta<0 \\
& \Rightarrow\left[\varphi_{\varepsilon}(\mathrm{y})\right]^{\prime}>-\Delta\left(\varphi_{\varepsilon}(\mathrm{y})\right)^{2} / \mathrm{k}=\left[\mathrm{g}_{\lambda}(\mathrm{y})\right]^{\prime}
\end{aligned}
$$

A desigualdade acima é sempre verdadeira, uma vez que $\left[\varphi_{\varepsilon}\left(\mathrm{y}_{\varepsilon \lambda}\right)\right]^{\prime} \geq 0$ e $\left[g_{\lambda}\left(y_{\varepsilon} \lambda\right)\right]^{\prime}<0$.

Portanto, a singularidade $\left(\mathrm{x}_{\varepsilon \lambda}, \mathrm{y}_{\varepsilon}\right)$ é uma sela hiperbólica de $\mathrm{Z}_{\varepsilon, \lambda}$, para $\varepsilon>0$ e $\lambda$ qualquer.

As figuras abaixo ilustram o campo vetorial descontínuo $Z_{\lambda}$ para os $\operatorname{casos} \lambda>0, \lambda<0, \sigma>0$ e $\sigma<0$. 


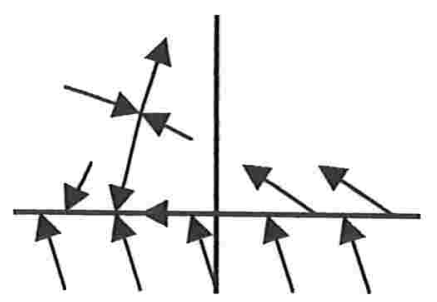

$\lambda<0$ e $\sigma>0$

Fig. 114

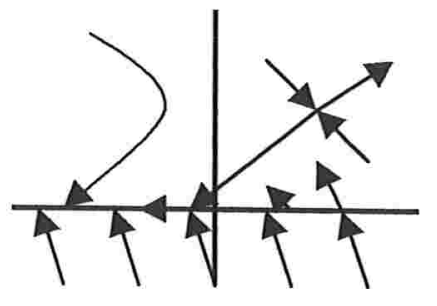

$\lambda<0$ e $\sigma<0$

Fig. 116

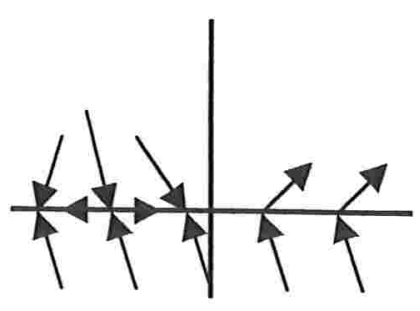

$\lambda>0$ e $\sigma>0$

Fig. 115

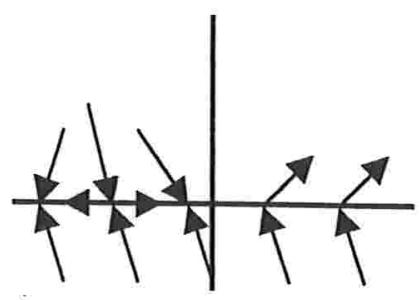

$\lambda>0$ e $\sigma<0$

Fig. 117

\section{Singularidade não Hiperbólica de $F_{Z}$}

Neste parágrafo será estudada a influência que uma singularidade não hiperbólica do campo $\mathrm{F}_{Z}$ tem sobre a família de campos vetoriais regularizados. Isto é feito utilizando-se uma família de campos vetoriais descontínuos $Z_{\lambda}=\left(X_{\lambda}, Y_{\lambda}\right)$ onde, para $\lambda=0$, o campo de Filippov $F_{Z}$ possui uma singularidade não hiperbólica, e para $\lambda \neq 0$, a singularidade do campo de Filippov é hiperbólica. A família utilizada será $Z_{\lambda}=\left(X_{\lambda}, Y_{\lambda}\right)$, onde $\mathrm{X}_{\lambda}(\mathrm{x}, \mathrm{y})=(-\mathrm{x}+\lambda,-1)$ e $\mathrm{Y}_{\lambda}(\mathrm{x}, \mathrm{y})=\left(\mathrm{x}^{2} / 2+\mathrm{x}, \mathrm{x}+1\right)$.

\subsection{Proposição}

Seja $Z_{\lambda}=\left(X_{\lambda}, Y_{\lambda}\right)$ a família definida acima. Então, para $\lambda=0$, o ponto $p=(0,0)$ é uma singularidade não hiperbólica de $F_{Z}$. Para $\lambda \neq 0, \lambda>-1, o$ campo $F_{Z}$ possui uma singularidade hiperbólica.

demonstração

Primeiro analisando-se o caso $\lambda=0$.

De fato, sendo $f(\mathrm{x}, \mathrm{y})=\mathrm{y}$, tem-se

$\mathrm{X}_{0} f(\mathrm{x}, \mathrm{y})=-1, \mathrm{Y}_{0} f(\mathrm{x}, \mathrm{y})=\mathrm{x}+1, \log 0 \mathrm{X}_{0} f(\mathrm{p})=-1<0, \mathrm{Y}_{0} f(\mathrm{p})=1>0$ 


$$
\begin{aligned}
& \operatorname{det}\left[X_{0}, Y_{0}\right](x, y)=-x^{2} / 2, \log 0 \operatorname{det}\left[X_{0}, Y_{0}\right](p)=0 . \\
& d\left(\left.\operatorname{det}\left[X_{0}, Y_{0}\right]\right|_{D}\right)(x, y)=-x, \log 0 d\left(\left.\operatorname{det}\left[X_{0}, Y_{0}\right]\right|_{D}\right)(p)=0 . \\
& d^{2}\left(\left.\operatorname{det}\left[X_{0}, Y_{0}\right]\right|_{D}\right)(x, y)=d^{2}\left(\left.\operatorname{det}\left[X_{0}, Y_{0}\right]\right|_{D}\right)(p)=-1 \neq 0 .
\end{aligned}
$$

Portanto, o ponto $(0,0)$ é uma singularidade não hiperbólica do campo $\mathrm{F}_{\mathrm{Z}}$ para $\lambda=0$.

Agora, analisando-se o caso $\lambda \neq 0$.

$$
\begin{aligned}
& \mathrm{X}_{\lambda} f(\mathrm{x}, \mathrm{y})=-1, \mathrm{Y}_{\lambda} f(\mathrm{x}, \mathrm{y})=\mathrm{x}+1, \log 0 \mathrm{Y}_{\lambda} f(\mathrm{x}, \mathrm{y})>0, \text { se } \mathrm{x}>-1 \\
& \operatorname{det}\left[\mathrm{X}_{\lambda}, \mathrm{Y}_{\lambda}\right](\mathrm{x}, \mathrm{y})=\lambda(\mathrm{x}+1)-\mathrm{x}^{2} / 2, \log 0 \operatorname{det}\left[\mathrm{X}_{\lambda}, \mathrm{Y}_{\lambda}\right](\mathrm{x}, \mathrm{y})=0 \Rightarrow \\
& \Rightarrow \lambda=\mathrm{x}^{2} /(2 \mathrm{x}+2) .
\end{aligned}
$$

$\mathrm{d}\left(\left.\operatorname{det}\left[\mathrm{X}_{\lambda}, \mathrm{Y}_{\lambda}\right]\right|_{\mathrm{D}}\right)(\mathrm{x}, \mathrm{y})=-\mathrm{x}+\lambda=-\mathrm{x}+\mathrm{x}^{2} /(2 \mathrm{x}+2) \neq 0$ se $\mathrm{x} \neq-2$.

Portanto, o ponto $(x, 0)$ é uma singularidade hiperbólica do campo $F_{Z}$ para $\lambda=\mathrm{x}^{2} /(2 \mathrm{x}+2)$, quando $\lambda \neq 0, \lambda>-1$.

\subsection{Proposição}

Dados $Z_{\lambda}=\left(X_{\lambda}, Y_{\lambda}\right)$ definido como acima, e uma função de transição $\varphi$, para cada $\varepsilon>0$, o campo vetorial regularizado $Z_{0, \varepsilon}$ possui uma singularidade tipo sela-nó.

demonstração

Para $\lambda=0$, a família de campos vetoriais regularizados é dada por

$$
\begin{aligned}
& \mathrm{Z}_{0 \varepsilon}(\mathrm{x}, \mathrm{y})=\left(1-\varphi_{\varepsilon}(\mathrm{y})\right)\left(\mathrm{x}^{2} / 2+\mathrm{x}, \mathrm{x}+1\right)+\varphi_{\varepsilon}(\mathrm{y})(-\mathrm{x},-1)= \\
& =\left(\mathrm{x}^{2} / 2+\mathrm{x}-\varphi_{\varepsilon}(\mathrm{y})\left(\mathrm{x}^{2} / 2+\mathrm{x}\right)-\mathrm{x} \varphi_{\varepsilon}(\mathrm{y}), \mathrm{x}+1-\varphi_{\varepsilon}(\mathrm{y})(\mathrm{x}+1)-\varphi_{\varepsilon}(\mathrm{y})\right)= \\
& =\left(\mathrm{x}^{2} / 2+\mathrm{x}-\varphi_{\varepsilon}(\mathrm{y})\left(\mathrm{x}^{2} / 2+2 \mathrm{x}\right), \mathrm{x}+1-\varphi_{\varepsilon}(\mathrm{y})(\mathrm{x}+2)\right)
\end{aligned}
$$

Para o ponto $(\mathrm{x}, \mathrm{y})$ ser uma singularidade de $\mathrm{Z}_{0 \varepsilon}$ deve-se ter

$$
\mathrm{Z}_{0 \varepsilon}(\mathrm{x}, \mathrm{y})=\left(\mathrm{x}^{2} / 2+\mathrm{x}-\varphi_{\varepsilon}(\mathrm{y})\left(\mathrm{x}^{2} / 2+2 \mathrm{x}\right), \mathrm{x}+1-\varphi_{\varepsilon}(\mathrm{y})(\mathrm{x}+2)\right)=(0,0)
$$


$\Rightarrow x=0$ e $\varphi_{\varepsilon}(y)=1 / 2$.

Portanto, a única singularidade de $Z_{0 \varepsilon}$ ocorre em $(x, y)=\left(0, \varphi_{\varepsilon}^{-1}(1 / 2)\right)$.

Investigando a natureza desta singularidade, tem-se

$\mathrm{DZ}_{0 \varepsilon}(\mathrm{x}, \mathrm{y})=\left[\begin{array}{ll}\mathrm{x}+1-\varphi_{\varepsilon}(\mathrm{y})(\mathrm{x}+2) & -\left[\varphi_{\varepsilon}(\mathrm{y})\right]^{\prime}\left(\mathrm{x}^{2} / 2+2 \mathrm{x}\right) \\ 1-\varphi_{\varepsilon}(\mathrm{y}) & -\left[\varphi_{\varepsilon}(\mathrm{y})\right]^{\prime}\end{array}\right]$

A função característica é dada por

$$
\begin{aligned}
& \left(\mathrm{x}+1-\varphi_{\varepsilon}(\mathrm{y})(\mathrm{x}+2)-\mu\right)\left(-\left[\varphi_{\varepsilon}(\mathrm{y})\right]^{\prime}-\mu\right)+\left[\varphi_{\varepsilon}(\mathrm{y})\right]^{\prime}\left(\mathrm{x}^{2} / 2+2 \mathrm{x}\right)\left(1-\varphi_{\varepsilon}(\mathrm{y})\right)=0 \\
& \Rightarrow \mu^{2}+\mu\left(\left[\varphi_{\varepsilon}(\mathrm{y})\right]^{\prime}-\mathrm{x}-1+\varphi_{\varepsilon}(\mathrm{y})(\mathrm{x}+2)\right)+\left[\varphi_{\varepsilon}(\mathrm{y})\right]^{\prime}\left(\varphi_{\varepsilon}(\mathrm{y})\left(-\mathrm{x}^{2} / 2-\mathrm{x}+2\right)+\right. \\
& \left.\mathrm{x}^{2} / 2+\mathrm{x}-1\right)=0
\end{aligned}
$$

No ponto $(\mathrm{x}, \mathrm{y})=\left(0, \varphi_{\varepsilon}{ }^{-1}(1 / 2)\right)$, tem-se

$\mu^{2}+\mu\left(\left[\varphi_{\varepsilon}(y)\right]^{\prime}-0-1+1 / 2(0+2)\right)+\left[\varphi_{\varepsilon}(y)\right]^{\prime}\left(1 / 2\left(-0^{2} / 2-0+2\right)+0^{2} / 2+0-1\right)=0$

$\Rightarrow \quad \mu^{2}+\mu\left[\varphi_{\varepsilon}(y)\right]^{\prime}=0$

$\Rightarrow \quad \Delta_{\mu}=\left(\left[\varphi_{\varepsilon}(\mathrm{y})\right]^{\prime}\right)^{2}>0$

$\Rightarrow \quad \mu=1 / 2\left(-\left[\varphi_{\varepsilon}(\mathrm{y})\right]^{\prime} \pm\left[\varphi_{\varepsilon}(\mathrm{y})\right]^{\prime}\right)$

$\Rightarrow \quad \mu_{1}=0$ e $\mu_{2}=-\left[\varphi_{\varepsilon}(y)\right]^{\prime}<0$

Portanto, a singularidade de $Z_{0 \varepsilon}$ é uma sela-nó.

Neste Capítulo foi iniciado o estudo das bifurcações dos campos vetoriais descontínuos. Há ainda muitos outros casos que pode ser investigados, contrariando-se cada uma das outras condições sobre os campos $X$ e $Y$ para pertençam ao conjunto $G^{r}(M)$ que não foram abordadas neste trabalho. 


\section{Conclusões}

Neste trabalho, seguindo a linha iniciada por Sotomayor e Teixeira em [S-T], foi dado tratamento para o conjunto de campos vetoriais descontínuos semelhante ao que é dado ao conjunto de campos vetoriais contínuos. Por isto, o primeiro passo foi fazer a caracterização de um conjunto de Campos Vetoriais Descontínuos cuja regularização resultasse numa família de campos vetoriais estruturalmente estáveis. Neste sentido, caminhou-se na direção de dar um significado à estabilidade estrutural de sistemas dinâmicos descontínuos. O conjunto obtido é um conjunto genérico.

Em seguida iniciou-se o estudo das bifurcações dos campos vetoriais descontínuos, no intuito de continuar seguindo o mesmo tratamento dado para os campos contínuos. Neste ponto tornou-se necessário acrescentar o parâmetro $\lambda$, formando uma família de Campos Vetoriais Descontínuos, de modo que a família de campos regularizados tornou-se uma família a dois parâmetros. Procurou-se, a título de exemplos, criar situações em que o campo descontínuo não pertence ao conjunto $G^{r}$, para $\lambda=0$. Estudou-se então a influência deste fato sobre a família de campos vetoriais regularizados. Em alguns casos ocorreu bifurcação, em outros não. O estudo das bifurcações pode continuar seguindo esta mesma linha, aqui iniciada, de tomar famílias de campos vetoriais descontínuos com elementos não pertencentes ao conjunto $G^{r}(M)$. 


\section{Bibliografia}

[A] A. Andronov, I. Gordon, E. Leontovich and G. Maier. Theory of Bifurcations of Dynamical systems on a Plane, Israel Program for Scientific Translations, John Wiley, New York, 1973.

[A-Ch] A. Andronov, V. Chaikin et al., Theory of Oscillators, Addison Wesley, 1966.

[B] R. G. Bartle, Elementos de Análise Real, Editora Campos, Rio de Janeiro, 1983.

[B-D] W. E. Boyce \& R.C. DiPrima, Elementary Differential Equation and Boundary Value Problems, John Wiley \& Sons Inc., $5^{\text {th }}$ Edition, 1992.

[F] A. L. Fernandes, Dobras e Cúspides em Sistemas Dinâmicos, Dissertação de Mestrado em Matemática Aplicada, I.M.E., USP, 1995.

[Fi] A. F. Filippov, Differential Equations with Discontinuous Right Hand Sides, Kluwer, 1988.

[K] V. S. Koslova, Roughness of a Discontinuous System, Vestnik Moskovskogo Universiteta, Matematika, no 5 , pp.16-20, 1984.

[L1] E. L. Lima, Curso de Análise, vol. I, Projeto Euclides, IMPA, Rio de Janeiro, 1976.

[L2] E. L. Lima, Curso de Análise, vol. 2, Projeto Euclides, IMPA, Rio de Janeiro, 1981 .

[M-M] M. C. Peixoto e M. M. Peixoto, Structural Stability in the Plane with Enlarged Boundary Conditions, Anais da Academia Brasileira de Ciências, 31(2), 1959

[P-M] J. Palis e W. de Melo, Introdução aos Sistemas Dinâmicos, Projeto Euclides, IMPA, Rio de Janeiro, 1978.

[P] P. Percell, Structural Stability in Manifolds with Boundary, Topology 12,1973 .

[Sh] M. M. Shumafov, Diffeomorphisms of Three-Dimensional Discontinuous Systems, Vestnik Moskovskogo Universiteta, Matematika, vol. $39, \mathrm{n}^{\circ} 6$, pp. $85-88,1984$. 
[S-T] J. Sotomayor e M.A. Teixeira, Regularization of Discontinuous Vector Fields, International Conference on Differencial Equation, pp. 207 a 223, Lisboa, 1995.

[S1] J. Sotomayor, Lições de Equações Diferenciais Ordinárias, Projeto Euclides, 1979.

[S2] J. Sotomayor, Curvas Definidas por Equações Diferenciais no Plano, Coloquio Brasileiro de Matemática, IMPA, Rio de Janeiro, 1981.

[S3] J. Sotomayor, Structural Stability in Manifolds with Boundary, in Global Analysis and its Applications, Vol. III, I.A.E.A, Viena, 1974.

[Sw] E. W. Swokowski, Cálculo com Geometria Analítica, vol. 2, Editora Makron, 1994.

[T] M. A. Teixeira, On Critical Points of Certain Planar Vector Fields, Anais da Academia Brasileira de Ciências, 56(3), 1984. 